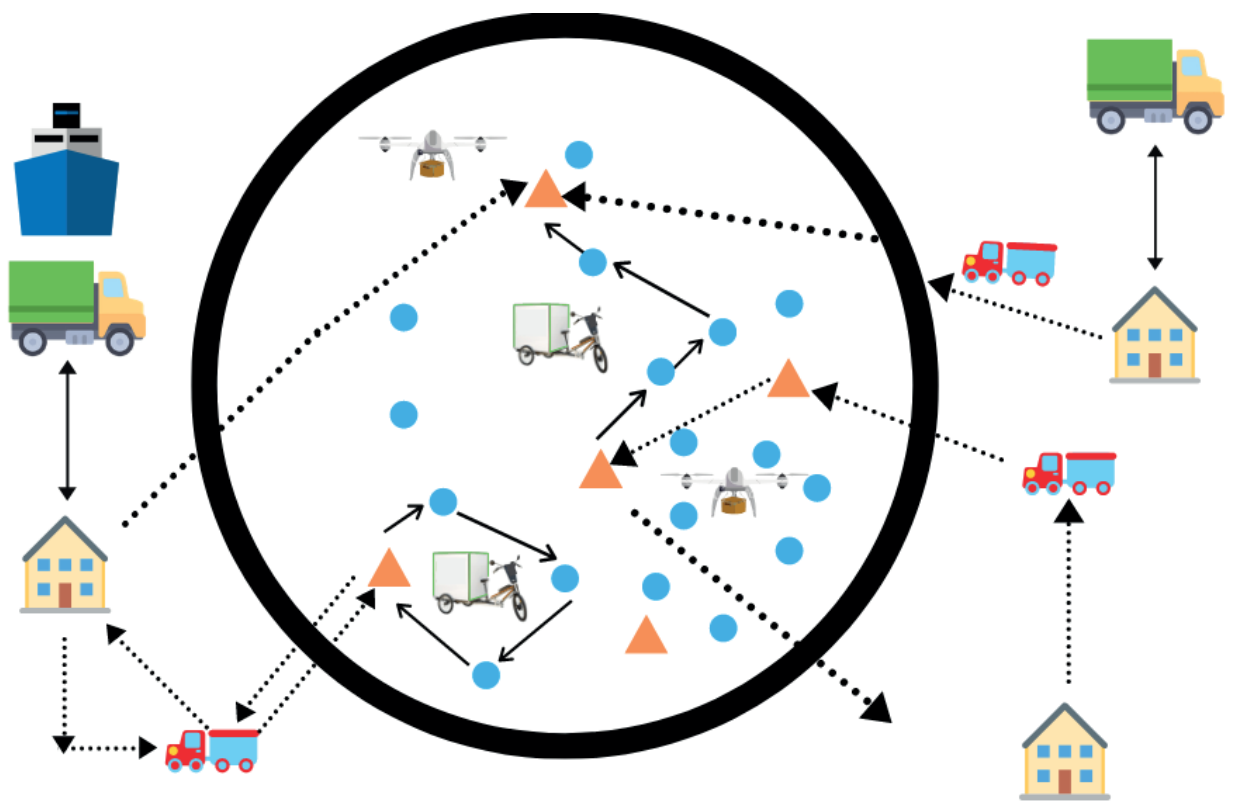

\title{
Introducción al problema de enrutamiento de vehículos en la logística de distribución
}

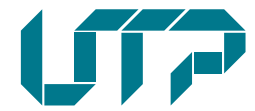
de Pereira

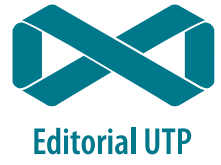

Eliana Mirledy Toro Ocampo John Fredy Castañeda Londoño Ramón Alfonso Gallego Rendón 
Eliana Mirledy Toro Ocampo, (Montenegro, Quindío, Colombia, 1970).

Ph.D en Ingeniería, Ingeniera Industrial de la Universidad Tecnológica de Pereira.

Profesora titular de la Facultad de Ciencias Empresariales de la Universidad Tecnológica de Pereira.

Coautora de los libros: Programación lineal y flujo en redes (2007), Técnicas Metaheurísticas de Optimización (2009), Técnicas Heurísticas Y Metaheurísticas (2015).

Ha publicado artículos en revistas especializadas nacionales e internacionales de su especialidad.

Pertenece al Grupo de investigación en Aplicaciones en Optimización y Procesos Estocásticos (GAOPE). elianam@utp.edu.co

John Fredy Castañeda Londoño, (Pereira, Risaralda, Colombia, 1984).

Magister en Investigación de Operaciones y Estadística, Ingeniero Electricista de la Universidad Tecnológica de Pereira, Estudiante del Doctorado en Ingeniería de La Facultad de Ingenierías de la Universidad Tecnológica de Pereira.

Ha publicado artículos en revistas especializadas nacionales e internacionales de su especialidad.

Pertenece al Grupo de investigación en Aplicaciones en Optimización y Procesos Estocásticos (GAOPE) y al Grupo de Planeamiento en Sistemas Eléctricos (GP).

jfcastaneda@utp.edu.co

Ramón Alfonso Gallego Rendón, (Circasia, Quindío, Colombia, 1957).

Ph. D en Ingeniería Eléctrica de la Universidad Estadual de Campinas UNICAMP-SP-Brasil, Ingeniero Electricista egresado de la Universidad Tecnológica de Pereira.

Profesor titular de la Facultad de Ingenierías de la Universidad Tecnológica de Pereira.

Coautor de los libros: Programación lineal y flujo en redes (2007), Programación lineal entera (2008), Técnicas Metaheurísticas de Optimización (2009), Modelos usados en el planeamiento de la expansión a largo plazo de sistemas de transmisión de energía eléctrica (2010), Técnicas Heurísticas y Metaheurísticas (2015), Flujo de carga en sistemas de transmisión (2016), Flujo de potencia óptimo en sistemas de transmisión de energía eléctrica (2019) entre otros.

Ha publicado artículos en revistas especializadas nacionales e internacionales de sus especialidades.

Pertenece al Grupo de Investigación Planeamiento en Sistemas Eléctricos (GP).

ragr@utp.edu.co 


\section{Introducción al problema de enrutamiento de vehículos en la logística de distribución}

Eliana Mirledy Toro Ocampo John Fredy Castañeda Londoño Ramón Alfonso Gallego Rendón

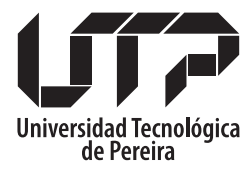

Colección Trabajos de Investigación

Facultad Ciencias Empresariales 


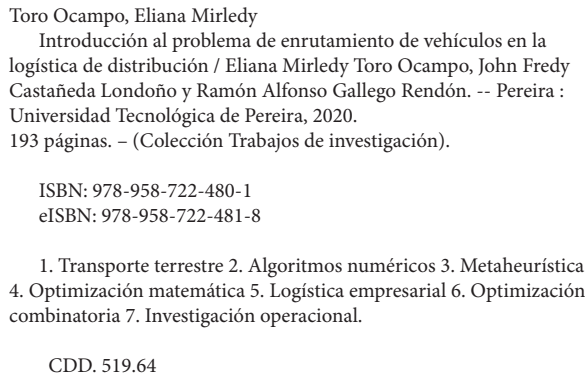

\section{(C) Eliana Mirledy Toro Ocampo \\ (C) John Fredy Castañeda Londoño \\ (C) Ramón Alfonso Gallego Rendón \\ Universidad Tecnológica de Pereira \\ Pereira, Colombia}

\section{Proyecto de Investigación}

Estudio del problema de localización y ruteo abierto aplicado a la logística de distribución en centros urbanos. Código CIE 7-19-1.

\section{Universidad Tecnológica de Pereira}

Vicerrectoría de Investigaciones, Innovación y Extensión

Editorial Universidad Tecnológica de Pereira

Pereira, Colombia

\section{Coordinador editorial:}

Luis Miguel Vargas Valencia

luismvargas@utp.edu.co

Teléfono 3137381

Edificio 9, Biblioteca Central "Jorge Roa Martínez"

Cra. 27 No. 10-02 Los Álamos, Pereira, Colombia

www.utp.edu.co

Montaje y producción:

David Restrepo Suarez.

Universidad Tecnológica de Pereira 



\section{CONTENIDO}

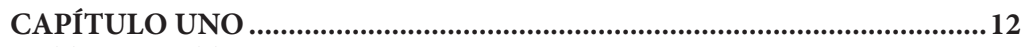

ANTECEDENTES ................................................................................................ 13

1.1. Enrutamiento de vehículos............................................................. 13

1.2. Clasicación de los problemas de enrutamiento....................................... 15

1.3. Funciones objetivo a considerar............................................................... 18

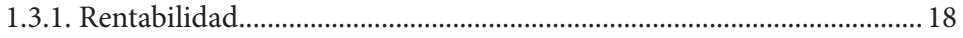

1.3.2. Calidad del servicio ................................................................................ 19

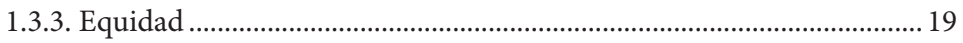

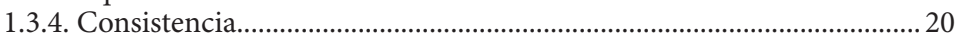

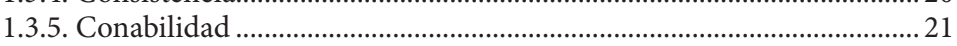

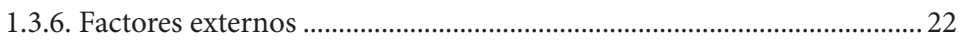

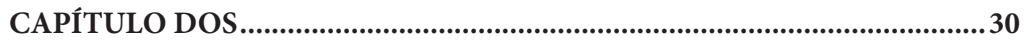

TÉCNICAS DE SOLUCIÓN PARA RESOLVER LOS VRP...............................31

2.1. Problema de enrutamiento de vehículos considerando restricciones. 32

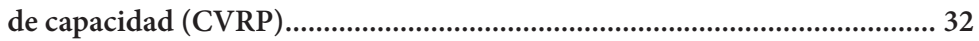

2.1.1. Técnicas exactas para resolver el CVRP.................................................. 32

2.1.2. Métodos heurísticos para resolver el CVRP............................................ 32

2.1.3. Adaptación de técnicas metaheurísticas para resolver el CVRP .......... 33

2.2. Problema de enrutamiento considerando múltiples depósitos.............. 34

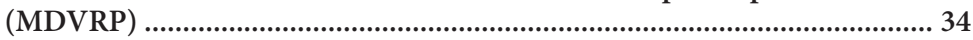

2.2.1. Técnicas exactas para resolver el MDVRP .............................................. 35

2.2.2. Técnicas heurísticas para resolver el MDVRP ........................................ 36

2.2.3. Técnicas metaheurísticas para resolver el MDVRP..................................36

2.3. Problema de localización de instalaciones con restricciones de .......... 38

capacidad (CFLP) ............................................................................................. 38

2.3.1. Técnicas heurísticas para resolver el CFLP.............................................. 39

2.3.2. Técnicas metaheurísticas para resolver el CFLP....................................... 39

2.4. Problema de localización y enrutamiento (CLRP) .................................. 40

2.4.1. Técnicas exactas para resolver el CLRP ………........................................ 40

2.4.2. Técnicas metaheurísticas para resolver el CLRP ..................................... 42

2.4.3. Técnicas math-heurísticas para resolver el CLRP ................................... 45

2.4.4. Planteamientos multiobjetivo para el LRP .............................................. 48

2.4.5. Aplicaciones del CLRP ............................................................................... 50

2.4.6. Planteamientos del problema Two-Echelon Vehicle Routing Problem

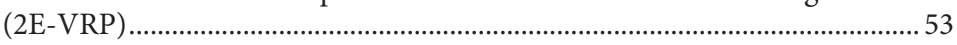

2.4.7. Aplicaciones del 2E-VRP ......................................................................... 55

2.5. Problemas de enrutamiento con ota propia y subcontratada .................56

2.5.1. Problema de enrutamiento de vehículos con ota propia y

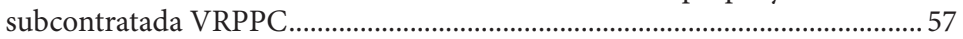

2.5.2. Problema multi-depósito de enrutamiento con ota propia y ota

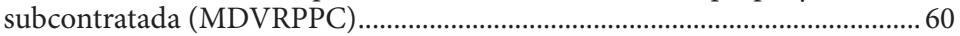

2.5.3. Problema de localización y enrutamiento con ota propia y

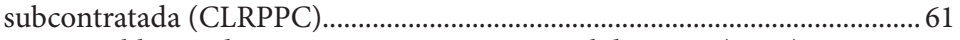

2.5.4. Problemas de enrutamiento sin retorno al depósito (open) ...................61 61

2.6. Optimización multiobjetivo........................................................................ 63

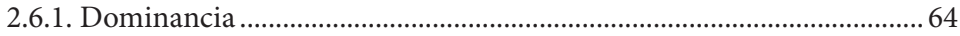

2.7. Enrutamiento de vehículos enfocado en logística de última milla...... 68 


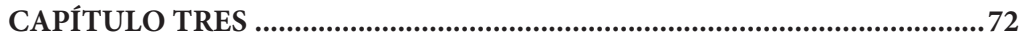

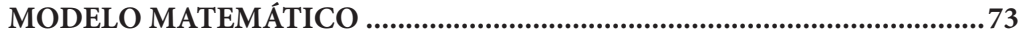

3.1. Marco de referencia.................................................................................... 73

3.1.1. Similitud de la operación en las redes de distribución de energía

eléctrica y los problemas de enrutamiento de vehículos.....................................73

3.1.2. Modelo matemático a ser propuesto ......................................................... 78

3.2. Problema de enrutamiento con ota propia y subcontratada ................... 79

3.2.1. Problema de localización y enrutamiento con ota propia y

subcontratada (CLRPPC)................................................................................ 79

3.2.2. Problema multidepósito considerando ota propia y subcontratada

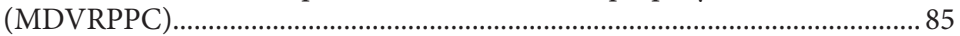

3.2.3. Problema de enrutamiento con ota propia y subcontratada

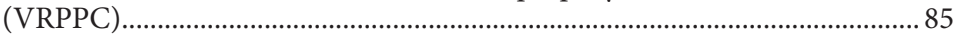

3.3. Problema de enrutamiento abierto ........................................................ 86

3.3.1. Problema de localización y enrutamiento abierto (OLRP) .................... 86

3.3.2. Problema de enrutamiento multidepósito abierto (MDOVRP) ..........88 88

3.3.3. Problema de enrutamiento abierto con único depósito (OVRP)......... 88

3.4. Problemas de enrutamiento de vehículos estándar ................................. 90

3.4.1. Problema de localización y enrutamiento estándar (CLRP)................ 90

3.4.2. Problema de enrutamiento multidepósito estándar (MDVRP) ........... 90

3.4.3. Problema de enrutamiento estándar (CVRP) ........................................... 92

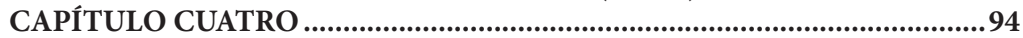

PLANTEAMIENTO DEL MODELO MATEMÁTICO DEL GCLRP Y

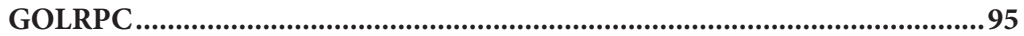

4.1. Cálculo del consumo de energía del vehículo ......................................... 95

4.2. Análisis de costos asociados a la función objetivo ................................... 99

4.3. Modelamiento matemático del problema GCLRP................................. 99

4.4. Formulación del problema GOLRP ......................................................... 101

CAPÍTULO CINCO .............................................................................................. 102

METODOLOGÍA DE SOLUCIÓN APROXIMADA ......................................103

5.1. Formulación multiobjetivo para el GVRPPCWE .................................. 104

5.2. Algoritmo Iterated Local Search (ILS)....................................................... 106

5.3. Descripción del algoritmo ILS multiobjetivo........................................ 109

5.4. Solución inicial local del ILS ..................................................................... 109

5.5. Random Variable Neighborhood Search (RVNS)................................. 111

5.5.1. Descripción del algoritmo para el RVNS................................................ 112

5.5.2. Metodología de búsqueda del algoritmo ILS multiobjetivo................. 113

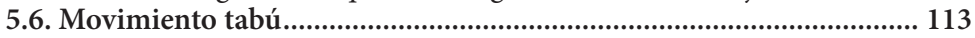

5.7. Operadores de intercambio inter-ruta....................................................... 114

5.8. Operadores de intercambio intra-ruta ................................................ 115

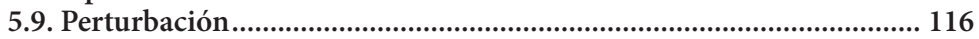

5.9.1. Criterio de distancia en movimientos de perturbación.........................117

5.10. Selección de soluciones no dominadas .................................................. 118

5.10.1. Descripción pseudocódigo: dominancia .............................................. 119

5.11. Procesamiento en paralelo ....................................................................... 119

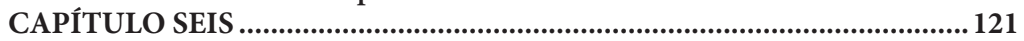

RESULTADOS COMPUTACIONALES......................................................... 122

6.1. Resultados de versiones VRP con ota propia y subcontratada............ 122

6.2. Resultados de las versiones VRP sin retorno al depósito ..................... 125

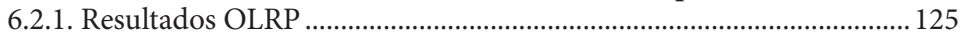

6.2.2. Resultados MDOVRP ........................................................................ 128

6.3. Resultados de versiones estándar del VRP .......................................... 133 
6.4. Resultados problemas CLRP considerando impacto ambiental ........ 139

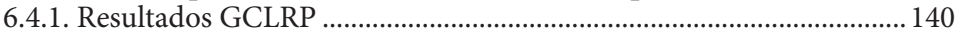

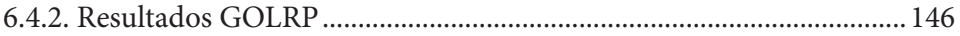

6.5. Análisis de la metodología aproximada para el GVRPPCWE ............ 154

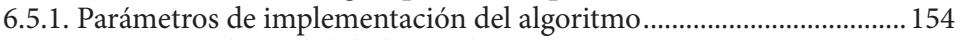

6.5.2. Vericación de estabilidad y rendimiento ................................................ 155

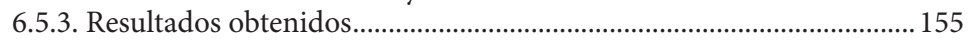

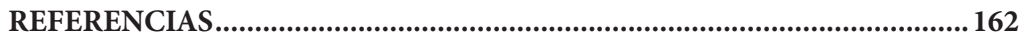




\section{FIGURAS}

Figura nro. 1. Tipos de VRP que pueden resolverse con el modelo matemático

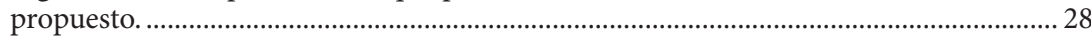

Figura nro. 2. Métodos de solución para los problemas de Enrutamiento........................ 35

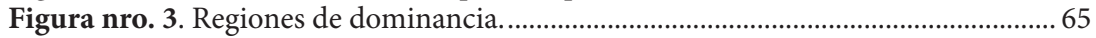

Figura nro. 4. Ejemplo hipervolumen en tres dimensiones. Cada punto genera un

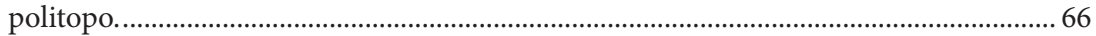

Figura nro. 5. Representación de los conjuntos A y R en dos dimensiones y las

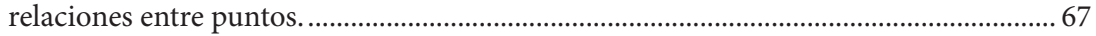

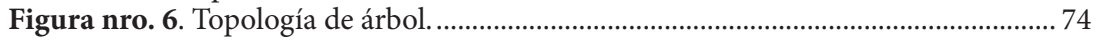

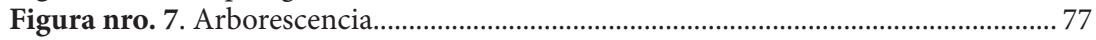

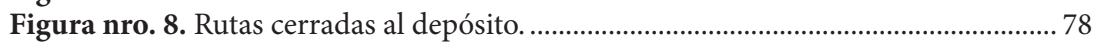

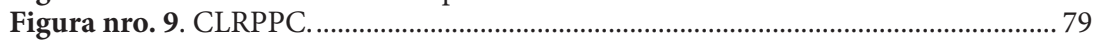

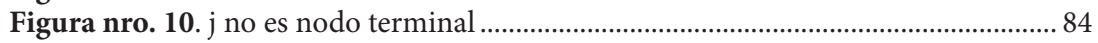

Figura nro. 11. j es nodo terminal................................................................................. 84

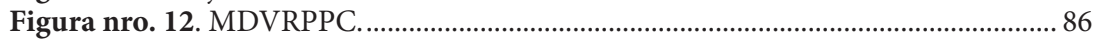

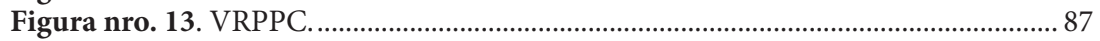

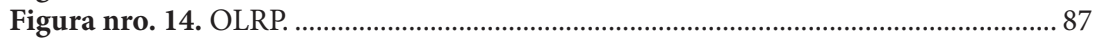

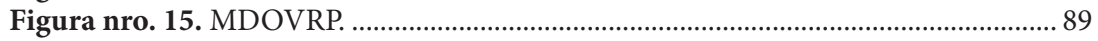

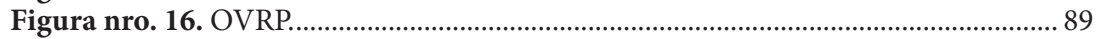

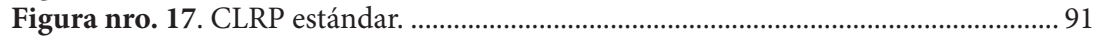

Figura nro. 18. MDVRP estándar ……….................................................................... 91

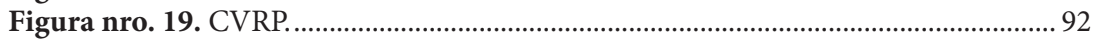

Figura nro. 20. Fuerzas actuando sobre el vehículo........................................................... 95

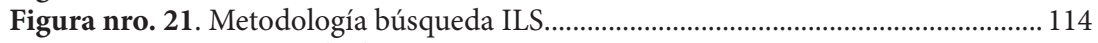

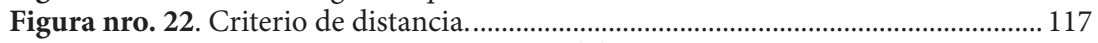

Figura nro. 23. Esquema procesamiento paralelo.......................................................... 119

Figura nro. 24. Solución óptima obtenida para el CLRPPC-50-5-2bbis........................ 128

Figura nro. 25. Solución óptima obtenida para el CVRPPC A-n39-k6 ........................ 129

Figura nro. 26. Solución óptima obtenida para pr08....................................................... 129

Figura nro. 27. Óptimo sin considerar restricción de longitud máxima de ruta

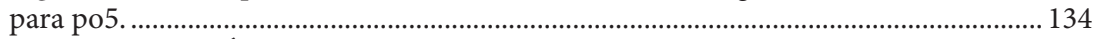

Figura nro. 28. Óptimo considerando restricción de longitud máxima de ruta para po5................................................................................................................... 138

Figura nro. 29. Solución óptima obtenida para el CVRP caso P-n101-k4 .................. 139

Figura nro. 30. Solución óptima obtenida para el CLRP del caso 100-5-1a................ 140

Figura nro. 31. G-CLRP 20-5. Solución costos operacionales $\Psi 1=4764$ USD; $\Psi 2$

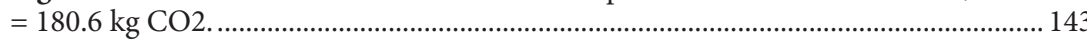

Figura nro. 32. G-CLRP 20-5. Solución considerando emisiones $\Psi 1=7790$ USD,

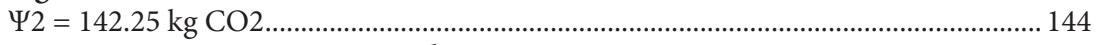

Figura nro. 33. G-CLRP 20-5. Solución min-max $\Psi 1=5727$ USD; $\Psi 2=153.45$

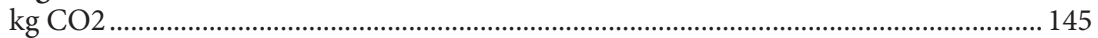

Figura nro. 34. G-CLRP 30-5. Solución costos operativos $\Psi 1=6106$ USD; $\Psi 2=$

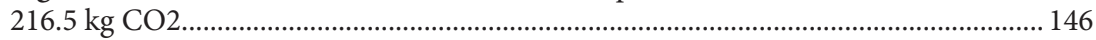

Figura nro. 35. G-CLRP 30-5. Solución considerando emisiones $\Psi 1=8496$ USD,

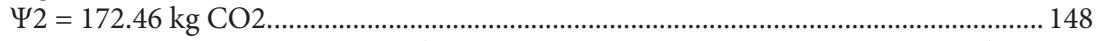

Figura nro. 36. G-CLRP 30-5. Solución $\min \max \Psi 1=7352$ USD; $\Psi 2=175.94 \mathrm{~kg}$

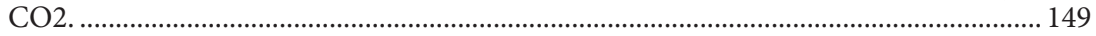

Figura nro. 37. Tres puntos del frente (costos, emisiones y min-max) ......................... 150 


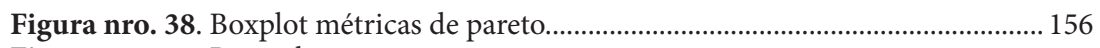

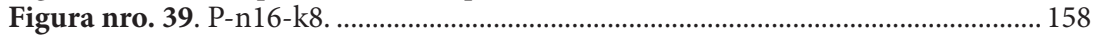

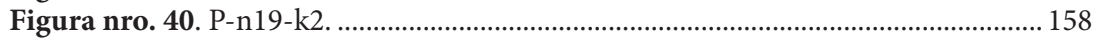

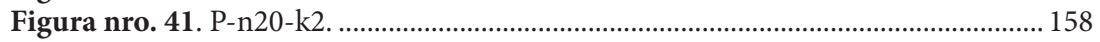

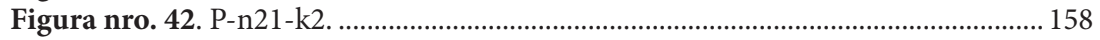

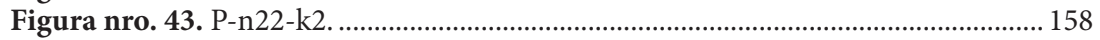

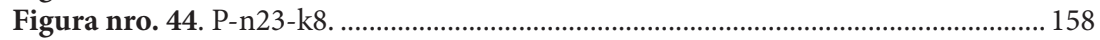

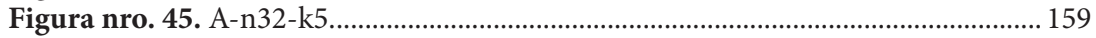

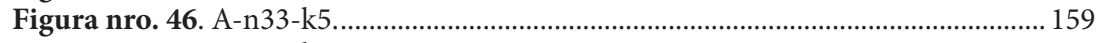

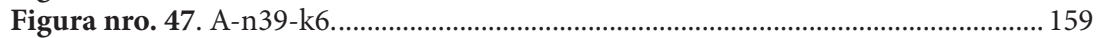

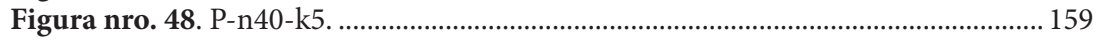

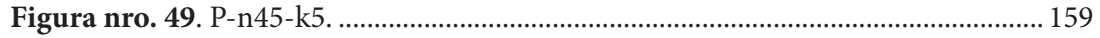

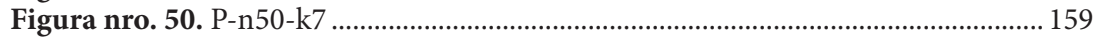

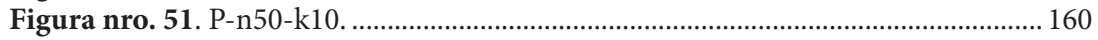

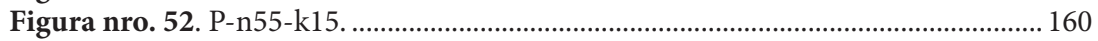

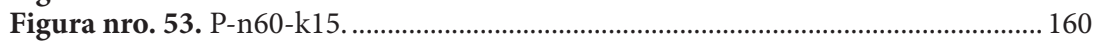

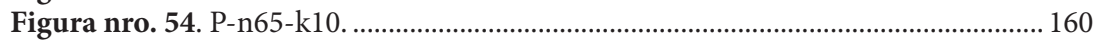

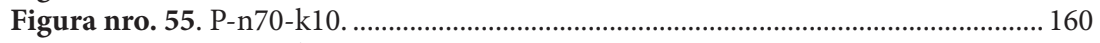

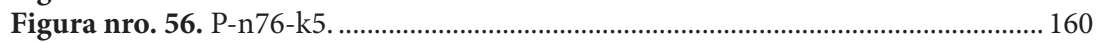

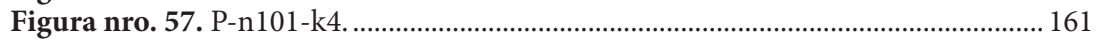

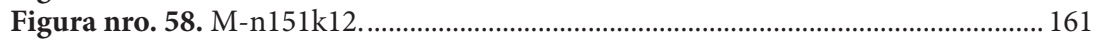

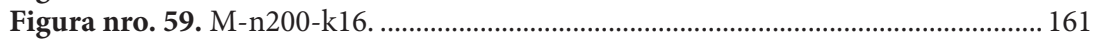




\section{TABLAS}

Tabla nro. 1. Características del VRP. .................................................................................. 17

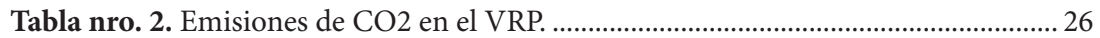

Tabla nro. 3. Nomenclatura del problema CLRPPC........................................................ 81

Tabla nro. 4. Parámetros del modelamiento matemático del problema GCLRP. ........ 100

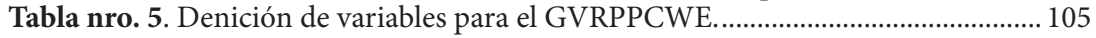

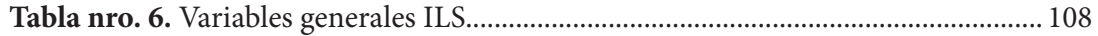

Tabla nro. 7. Variables solución inicial local ILS............................................................ 110

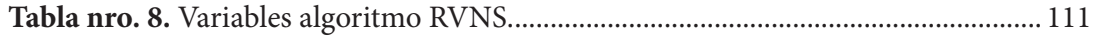

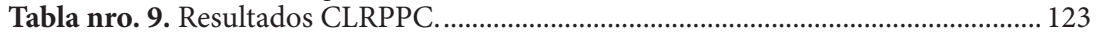

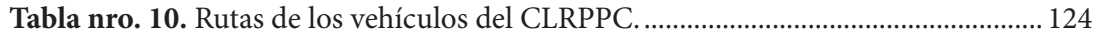

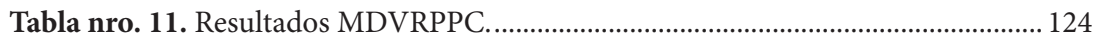

Tabla nro. 12. Rutas de los vehículos del MDVRPPC. ………........................................ 125

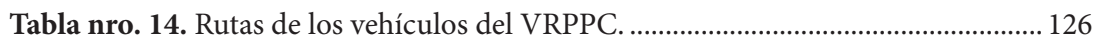

Tabla nro. 13. Resultados VRPPC...................................................................................... 127

Tabla nro. 15. Resultados computacionales del OLRP sobre los casos de Prins et

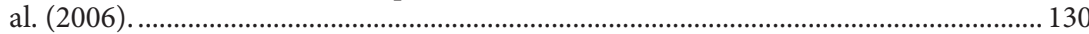

Tabla nro. 16. Resultados computacionales para los casos en Tuzun y Burke

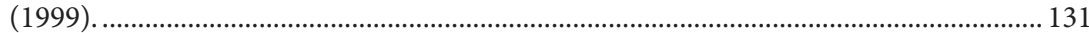

Tabla nro. 17. Resultados computacionales obtenidos por los modelos

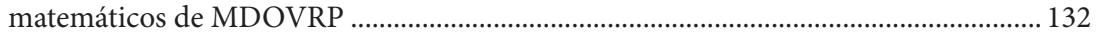

Tabla nro. 18. Resultados con y sin restricción de distancia para el MDOVRP........... 133

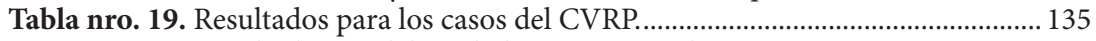

Tabla nro. 20. Resultados modelo radial y MTZ para el MDVRP. .................................. 136

Tabla nro. 21. Resultados de los casos del CLRP ...................................................... 137

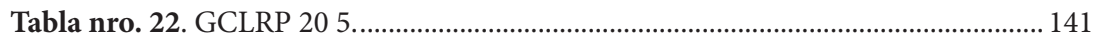

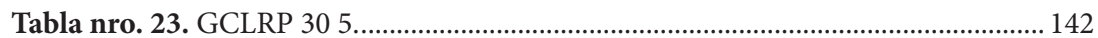

Tabla nro. 24. Resultados computacionales del G-CLRP 20-5 ..................................... 142

Tabla nro. 25. Resultados computacionales G-CLRP 30-5 ......................................... 143

Tabla nro. 26. Resultados computacionales para los casos tomados de Prins et al.

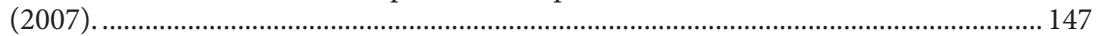

Tabla nro. 27. Resultados computacionales del GOLRP usando Prins et al.

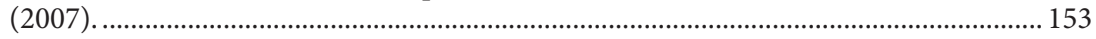

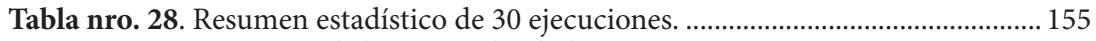

Tabla nro. 29. Instancias de baja complejidad matemática.............................................. 157

Tabla nro. 30. Instancias de alta complejidad matemática............................................... 157 


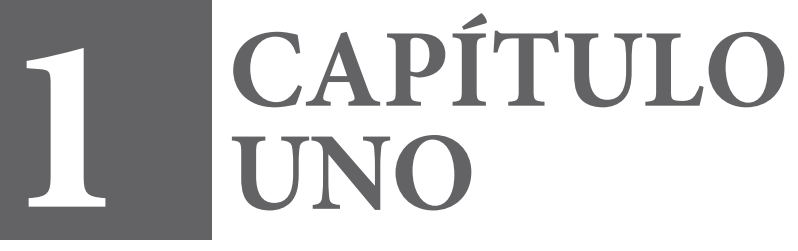




\section{ANTECEDENTES}

\subsection{Enrutamiento de vehículos}

En el desarrollo de la actividad humana los problemas de enrutamiento han despertado interés tanto en la comunidad académica como empresarial por la gran cantidad de aplicaciones logísticas en diferentes tipos de organización: gubernamentales, militares y empresariales (Bektas et al., 2014). En la literatura especializada esta temática ha sido denominada como Vehicle Routing Problem (VRP). Con base en las aplicaciones de cada ambiente se redefine una gran diversidad de variantes, lo que ha generado un número importante de publicaciones en esta área del conocimiento (Braekers et al., 2016).

La gestión eficiente de la cadena de suministro abarca tanto la distribución de materias primas, desde sus fuentes generadoras hasta los centros de procesamiento, así como la distribución de bienes y servicios hasta los centros de distribución y de allí hacia los clientes finales. En el análisis del transporte a través de la cadena de suministro, los centros urbanos constituyen las mayores aglomeraciones de capital manufacturado debido a que sus infraestructuras regulan los flujos de energía en las actividades económicas, los edificios y el transporte, esto hace que la infraestructura física de las ciudades, incluida la del transporte, configuren los patrones de todo tipo de consumo. Se considera que las áreas urbanas consumen alrededor del $75 \%$ del suministro de energía mundial y se espera que siga aumentando debido al crecimiento urbano (Chowdhury et al., 2019).

Por otro lado, se deben tener en cuenta diferentes indicadores de desempeño, asociados al transporte de insumos y los productos finales en la cadena de suministro, ya que dichos indicadores ayudan a determinar la calidad de las soluciones halladas, así como también el nivel del servicio prestado y de la satisfacción del cliente. Entendiéndose el nivel de servicio prestado como el objetivo de la empresa, el cual es calculado mediante el análisis de indicadores de servicio, derivados de la actividad de la empresa y donde la satisfacción del cliente es la percepción que se tiene sobre determinado producto y/o empresa antes, durante y después de conocerla; este es medido mediante 
Introducción al problema de enrutamiento de vehículos en la logística de distribución

herramientas de estudio de mercado, tales como encuestas y entrevistas, las cuales brindan un enfoque de la parte comercial y de mercadeo de una organización. Los indicadores que se pueden considerar para medir el nivel de desempeño pueden ser:

- Costo por kilómetro (km): Este determina el costo de conducir un vehículo un kilómetro y se calcula como se muestra en 1.1 .

$$
\text { Costo por } k m=\frac{\text { Costo del conductor }+ \text { Costo del combustible }+ \text { Costo del vehículo }}{\text { Distancia total }}
$$

- Uso de capacidad de flota: Determina cuál fue el aprovechamiento de la capacidad total y su cálculo está representada en 1.2 .

$$
\text { Uso de capacidad de flota }=\frac{\text { Capacidad utilizada }}{\text { Capacidad total }}
$$

- Arcos por viaje y kilómetros por arco: Dado que un arco es el recorrido entre un depósito y un nodo, o entre dos nodos. Estos indicadores determinan el número de arcos realizados por viaje y el número de kilómetros por arco, y se calculan como se muestra en 1.3 y 1.4 , respectivamente.

$$
\begin{aligned}
& \text { Arcos por viaje }=\frac{\text { Cantidad de nodos visitados }}{\text { Cantidad de viajes }} \\
& \text { Kilmetros por arcos }=\frac{\sum\{\text { Distancia del viaje }\}}{\text { Cantidad de arcos }}
\end{aligned}
$$

- Tiempo de descargue promedio: Este indicador mide el tiempo promedio que tarda hacer un descargue en cada punto como se muestra en 1.5

Tiempo de descargue promedio $=\frac{\text { Tiempo total de descargue }}{\text { Cantidad de entregas }}$ 
- Paradas planeadas contra paradas reales: Este determina el número de paradas reales con respecto a las paradas planeadas como se muestra en 1.6.

$$
\text { Paradas planeadas vs reales }=\frac{\text { Cantidad de paradas reales }}{\text { Cantidad de paradas planeadas }}
$$

La generación de rutas eficientes se constituye en un elemento de relevancia en la operación de cualquier tipo de organización, por tanto el estudio del problema de enrutamiento de vehículos puede entregar soluciones, que minimicen los costos de operación así como sus implicaciones directas en la reducción del consumo de combustible los cuales impactan directamente la minimización de efectos contaminantes, medidos como kilogramos de $\mathrm{CO}_{2}$. Este enfoque está en concordancia con la filosofía actual de las empresas donde se intenta encontrar soluciones sostenibles desde el punto de vista económico y ambiental con la óptica de minimizar la huella ecológica, producida por las actividades empresariales realizadas.

\subsection{Clasificación de los problemas de enrutamiento}

En una temática tan amplia y relevante como el enrutamiento de vehículos es necesario consolidar sistemas de información donde se documente el avance en la temática desde el tipo de aplicaciones reales, hasta los modelos matemáticos y las metodologías de solución propuestas. En esta dirección, Reisman (1992) indica que, para realizar una contextualización del estado del arte en cualquier temática, es necesario consolidar una taxonomía o un marco generalizado donde se resuman las variantes del problema. La taxonomía debe ser sencilla de forma que comunique, enseñe y almacene la literatura disponible para que, con base en ella, se identifiquen posibles líneas de investigación o vacíos en el conocimiento que merezcan ser atendidos. Los modelos y estrategias de solución del VRP pueden ser transversales a otros problemas debido a que estos pueden ser representados a través de una estructura de red. Por tanto, alguna contribución en esta temática puede ser válida para representar y resolver problemas de redes de distribución de energía eléctrica o de la distribución de otros servicios públicos como agua, gas, combustibles o de las redes cableadas de comunicaciones.

En el campo del enrutamiento de vehículos se han realizado diferentes tipos de aproximaciones. Es relevante mencionar la propuesta de Eksioglu et al. (2009), representada en la Tabla nro. 1 donde se consideran algunas características del escenario como:

- Restricciones asociadas a la división de la carga. 
Introducción al problema de enrutamiento de vehículos en la logística de distribución

- Tiempos de atención en cada nodo o cliente.

- Estructura de la ventana de tiempo.

- Clasificación de los nodos: entrega, recogida, entrega y recogida.

- Consideración de efectos ambientales.

- Número de paradas en la ruta.

- Cantidad demandada por el cliente.

- Horizonte de tiempo.

- Condición de cubrimiento de subconjuntos de clientes.

La Tabla nro. 1, además, contiene información de las características físicas del problema tales como:

- Localización de los clientes.

- Número de paradas.

- Número de vehículos.

- Naturaleza de los vehículos.

- Homogeneidad de los vehículos.

- Costos de transporte.

- Diseño de la red.

- Localización geográfica de los clientes.

- Tipos de ventanas de tiempo.

- Restricciones de capacidad de los vehículos.

- Tiempos de viaje.

- Elementos a transportar. 


\begin{tabular}{|c|c|c|}
\hline 1. Tipo de estudio & 2.8.2 Entregas o recogidas. & 3.10 Duración del viaje \\
\hline 1.1 Modelos matemáticos & 2.9 Atención a nodos o a arcos & 3.10.1 Determinístico \\
\hline 1.2 Métodos de solución & 2.9.1 Consideración de precedencias & 3.10.2 Dependiente del tiempo \\
\hline 1.2.1 Exactos & 2.9.2 Atención de subconjuntos & 3.10.3 Aleatorio \\
\hline 1.2.2 Heurísticas y metaheurísticas & 2.9.3 Bidireccional & 3.10.4 De acuerdo al tráfico \\
\hline 1.2.3 Math-heurísticas & 2.10 Efectos contaminantes & 3.11 Fletes \\
\hline 1.2.4 Sim-heurísticas & 2.10.1 Frentes multiobjetivo eficientes & 3.11.1 Basado en el tiempo de viaje \\
\hline 1.2.4 Soluciones en tiempo real & 2.10.2 Mono-objetivo ponderado & 3.11.2 Basado en la distancia \\
\hline 1.3 Metabuscadores & 3. Características físicas & 3.11.3 Dependiendo del vehículo ${ }^{4}$ \\
\hline 2. Características del escenario & 3.1 Diseño de red de transporte & 3.11.4 Función de tardanza \\
\hline 2.1 Paradas en la ruta & 3.1.1 Grafo dirigido & 3.11.5 Basado en la operación \\
\hline 2.1.1 Parcialmente conocida-probabilística & 3.1.2 Grafo bidireccional & 3.11.6 Emisiones contaminantes \\
\hline 2.1.2 Determinístico & 3.2 Ubicación de clientes & 3.11.7 Costos de mantenimiento \\
\hline 2.2 División de la carga & 3.2.1 Enrutamiento de arcos & 3.11 .8 Implica riesgos \\
\hline 2.2.1 División permitida & 3.2.2 Clientes sobre los nodos & 3.12 Tipo de carga \\
\hline 2.2 .2 Indivisible & 3.3 Ubicación geográfica de los clientes & 3.12.1 Mercancías \\
\hline 2.3 Demanda del cliente & 3.3.1 Rural (aleatoriamente dispersa) & 3.12.2 Personas \\
\hline 2.3.1 Aleatorio & 3.3.2 Urbana dispersa mediante un patrón & 3.12.3 Cuadrillas de servicios \\
\hline 2.3.2 Determinístico & 3.3.3 Mixto & 4. Características de la información \\
\hline 2.3.3 Desconocido ${ }^{1}$ & 3.4 Puntos de carga/descarga & 4.1 Naturaleza de los datos \\
\hline 2.4 Tiempos de nuevos clientes & 3.4.1múltiples depósitos & 4.1.1 Parcialmente dinámico \\
\hline 2.4.1 Desconocido & 3.4.2 Único depósito & 4.1.2 Estático \\
\hline 2.4.2 Aleatorios & 3.6 Clase de ventanas de tiempo & 4.2 Tipo de datos \\
\hline 2.4.3 Determinísticos & 3.6.1 Sobre las vías & 4.2.1 Aleatoria \\
\hline 2.5 Sitio del servicio/tiempo de espera & 3.6.2 Sobre los clientes & 4.2.2 Conocida (Determinística) \\
\hline 2.5.1 Dependiente del tiempo & 3.6.3 Sobre los conductores & 4.2.3 Desconocida (Tiempo real) \\
\hline 2.5.2 Determinístico & 3.6.4 Sobre los depósitos & 4.2.4 Pronosticada \\
\hline 2.5.3 Aleatorio & 3.7 Tamaño de la flota & 4.3 Accesibilidad de los datos \\
\hline 2.5.4 Congestión vehicular & 3.7.1 Uno & $4.3 .1 \mathrm{Global}$ \\
\hline 2.5.5 Desconocido & 3.7.2 Flota ilimitada & 4.3 .2 Local \\
\hline 2.6 Estructura de la ventana de tiempo & 3.7.3 Número fijo de vehículos & 4.4 Procesamiento de la información \\
\hline 2.6.1 De estricto cumplimiento & 3.8 Capacidad del vehículo & 4.4.1 Descentralizada \\
\hline 2.6.2 Relajada & 3.8.1 Capacidad infinita & 4.4.2 Centralizada \\
\hline 2.6 .3 combinada & 3.8.2 Capacidad estricta & 5. Naturaleza de la Información \\
\hline 2.7 Horizonte de tiempo & 3.9 Categoría de la flota & 5.1 Tipo de información \\
\hline 2.7.1 Varios periodos & 3.9.1 Flota homogénea & 5.1.1 Información real \\
\hline 2.7.2 Periodo único & 3.9.2 Flota heterogénea & 5.1.2 Información teórica \\
\hline 2.8 Naturaleza de los nodos & 3.9 .3 vehículos de carga específica ${ }^{2}$ & 5.1 .3 teórica y real \\
\hline 2.8.1 Entregas y recogidas & 3.9.4 Adaptación al cliente ${ }^{3}$ & 5.2 No usan datos \\
\hline
\end{tabular}

${ }^{1}$ Se toman los datos en el momento especifico de operación a través de sistemas de información en tiempo real como GPS.

${ }^{2}$ Vehículos específicos por tipo de carga.

${ }^{3}$ El cliente requiere un vehículo con características específicas.

${ }^{4}$ Variación en el costo de operación del vehículo, pueden ser vehículos que usan combustibles fósiles, energía eléctrica o propulsión humana.

TABLA NRO. 1. Características del VRP.

Elaboración propia. 


\subsection{Funciones objetivo a considerar}

La función objetivo a considerar en los abordajes actuales no solo corresponde a la clásica minimización de costos. Según Vidal et al. (2020), se pueden identificar diferentes objetivos, medidos como una etapa dentro de la optimización aplicada para dar solución al VRP:

\subsubsection{Rentabilidad}

En gran cantidad de aplicaciones del problema de enrutamiento, la optimización de costos es el principal objetivo. Este puede incluir costos fijos por ruta (costo del vehículo, seguros, salarios), al igual que costos variables, proporcionales a las distancia y duración del viaje. En algunas circunstancias, la rentabilidad debe se extendida a otros factores como se ilustra a continuación:

- Índices de rendimiento: En los problemas que consideran un horizonte continuo como en el caso de los problemas de enrutamiento donde se considera el inventario, es necesario optimizar la relación entre el costo de enrutamiento y la cantidad entregada en el horizonte de planificación (Archetti et al., 2017).

- Beneficios: En diferentes escenarios la minimización de costos compite con la maximización de ganancias, en las decisiones comerciales tácticas, por ejemplo, cuando se da peso a algunos clientes, es representado por un premio cuando es atendido. Allí, el objetivo es maximizar la ganancia total medida como la diferencia entre los premios recaudados y los costos de enrutamiento. Esto da lugar a un VRP con ganancias (Archetti et al., 2014). Dentro de las aplicaciones se encuentran el reconocimiento militar (Mufalli et al., 2012) y el diseño de itinerarios para turismo (Vansteenwegen et al., 2009).

- Subcontratación: Cuando la operación de las organizaciones se ve afectada por altos volúmenes de entrega es necesario subcontratar parte de la operación porque la flota propia de vehículos es insuficiente para atender la demanda. Esta situación ha llevado a la definición del problema de enrutamiento considerando la flota propia y la subcontratada (Toro-Ocampo et al., 2016). En esta situación es posible considerar tres tipos de costos para definir la forma de realizar la subcontratación de forma directa: por tramo recorrido, considerando tarifa plana por día o según el flujo según la distancia y el peso (Krajewska y Kopfer, 2009). En Dabia et al. (2019) se considera una función no lineal en la definición de la función objetivo, asociada a descuentos por volumen de la mercancía transportada. 


\subsubsection{Calidad del servicio}

El servicio y las condiciones que el cliente requiere son un factor de diferenciación de gran relevancia en los mercados actuales. Este tipo de valor es altamente apreciado en operaciones humanitarias, en el transporte público y en la atención logística hospitalaria en casa.

- Objetivos acumulativos: En algunas aplicaciones del problema de enrutamiento, la función objetivo corresponde a la suma de tiempos individuales de llegada a cada cliente. Este tipo de métrica es usado en operaciones de asistencia en emergencia o en situaciones de auxilio a un conjunto de nodos, el servicio es considerado como el aspecto más relevante para los cuerpos de socorro en situaciones de desastre (Campbell et al., 2008). El objetivo acumulativo cuando es ponderado puede adaptarse en rutas donde el cliente es el centro de la operación logística (Huang et al., 2012)

- Medidas de inconveniencia: En el transporte de pasajeros, la planificación de rutas es especialmente relevante en el enrutamiento de autobuses escolares (Ellegood et al., 2020) y en los servicios de atención médica a domicilio o en el servicio de taxis a domicilio, las medidas que el cliente valora son la minimización del tiempo de espera y la minimización del tiempo máximo de viaje. Esta medición indica la atención oportuna del cliente (Beirigo et al., 2019; Liang et al., 2020).

- Niveles del servicio: Una tendencia común en muchas organizaciones es subcontratar las actividades logísticas de distribución mediante operadores logísticos denominados. Para garantizar el nivel de servicio en las negociaciones, se definen proporciones mínimas de entregas a tiempo. En algunos casos, se consideran los niveles de servicio como restricciones suaves y existen penalidades por el incumplimiento. En aplicaciones como la atención médica domiciliaria, el objetivo principal, por ejemplo, es el cumplimiento del servicio (Fathollahi-Fard et al., 2020). En Orlis et al. (2020), se describe la automatización para realizar reposición de cajeros automáticos.

\subsubsection{Equidad}

En muchos casos, las soluciones eficientes no son necesariamente equitativas; es necesario evaluar la distribución justa de recursos, beneficios y responsabilidades. La equidad de ruta puede medirse con diferentes ópticas tal como lo sugieren Matl et al. (2019), a saber:

- Equilibrio de la carga de trabajo: Generalmente este equilibrio está asociado con el número de horas que laboran los conductores o el personal que proporciona el servicio. El objetivo entonces es minimizar la diferencia entre 
la ruta más larga y la ruta más corta. Esta medición se hace generalmente con base en el tiempo que se requiere para realizar la ruta o en la duración del servicio (Galindres-Guancha et al., 2018). Algunos ejemplos reportados en la literatura son: carga de trabajo de técnicos de servicio (Blakeley et al., 2003), carga de trabajo de médicos a domicilio (Liu, 2013), atención de territorios (Bruno et al., 2017).

- Equidad de servicio: Este tipo de métrica es de interés en la gestión del transporte público y de la logística humanitaria. Cuando se lleva a cabo la atención de desastres, puede aparecer diferencia entre la calidad de la cobertura y la equidad. Un ejemplo de esta situación se da cuando hay suministros suficientes para atender una comunidad pero la puntualidad en la entrega puede ser desigual. Situaciones de este tipo están discutidas en Huang et al. (2012).

- Planificación colaborativa: En ambientes tan competidos como el actual donde hay fuertes presiones en cuanto a los valores del fletes y márgenes de contribución estrechos en el sector de la logística, los transportadores deciden realizar colaboraciones horizontales para aumentar su capacidad de gestión (Gansterer y Hartl, 2018). En estas situaciones, el objetivo es generar rutas que minimicen el costo individual y, por tanto, el beneficio de esa reducción debe ser repartido de forma equitativa entre los socios que realizan la colaboración en relación con los recursos aportados (Guajardo y Rönnqvist, 2016).

\subsubsection{Consistencia}

En el diseño de rutas, solo pensar en el costo óptimo se constituye en un error debido a que cuando se reoptimizan los procesos puede ser que aparezcan nuevas rutas que afectan la curva de aprendizaje de los conductores gracias a que surge un cambio en sus itinerarios, factor que afecta la eficiencia en el desarrollo de la ruta (Kovacs et al., 2014). Otro elemento que se ve afectado es la personalización de la atención a los clientes. Por tal motivo, en muchas ocasiones, es preferible diseñar rutas consistentes con respecto a varias métricas durante múltiples periodos de tiempo.

- Consistencia temporal: El momento de prestación del servicio es un aspecto valorado por muchos clientes. En esta dirección, el objetivo es atender el cliente en un intervalo de tiempo semejante todos los días. Este tipo de métrica, por tanto, se aplica en problemas de enrutamiento de múltiples periodos. Una alternativa para cumplir este objetivo es considerar dentro de la operación ventanas de tiempo auto-impuestas que se comunican al cliente para generar planes de enrutamiento, respetando estos compromisos durante el horizonte de planificación (Jabali et al., 2015).

- Orientada a la persona: En el problema específico de enrutamiento de servicios médicos en horizonte de tiempo periódico, se ha observado que el cliente prefiere ser atendido por el mismo funcionario, debido a que se 
establecen las relaciones personales entre el servidor y el cliente. Este tipo de coherencia es muy conveniente en la asistencia médica donde la calidad del servicio se mide por el conocimiento de las condiciones del paciente por parte del personal sanitario (Eveborn et al., 2006). Una forma de medir este objetivo es maximizar el número de visitas que un conductor visita algún cliente o conjunto de clientes (Smilowitz et al., 2013).

- Regional o de entrega: La curva de aprendizaje de los conductores en cuanto al conocimiento de las rutas es evidente cuando realizan recorridos similares, debido a que pueden gestionar de forma eficiente condiciones de tráfico, rutas más cortas, desvíos, entre otras (Holland et al., 2017).

Por tanto, es deseable tener continuidad en la asignación de rutas a determinados funcionarios. La función objetivo puede ser definida como la maximización de nodos visitados en cada ruta dentro de una distancia definida (Sungur et al., 2010) o como la maximización del número de veces que el conductor visita repetidamente el mismo conjunto de clientes (Smilowitz et al., 2013).

- Consistencia de entrega: En algunas situaciones, el problema de enrutamiento va ligado a la gestión de inventarios en horizontes de tiempo periódicos. En este contexto, es deseable programar entregas a un nivel constante, en cada visita. Una forma de garantizar este aspecto es restringir las cantidades de entrega para cada cliente dentro de límites, con topes mínimos y máximos (Coelho et al., 2012).

\subsubsection{Confiabilidad}

En la medida en que se requiere mostrar el VRP en escenarios reales, aparecen variaciones en los valores de demanda, condición frecuente en los ambientes cotidianos. Esto representa un desafío para realizar la planeación de las rutas; en general, cuando se resuelve el problema se considera este parámetro como un valor determinístico. Si se contempla esta situación aparece el problema de enrutamiento estocástico, capacitated vehicle routing problem with stochastic demands.

En el evento de aparición de fallos de ruta, es necesario implementar una acción de recurso que minimice los mismos. Bertsimas (1992) propone una política de recurso con base en retornos al depósito en los casos donde el vehículo no tenga suficiente carga para satisfacer la demanda del cliente en algún nodo de la ruta; después de un reabastecimiento, el vehículo retorna al cliente que no pudo satisfacer y sigue con la ruta planeada. En Birge y Louveaux (2011) se plantean dos estrategias adicionales para la solución del VRPSD. La primera estrategia «espere y observe» se basa en conocer con antelación la demanda de cada cliente para programar la ruta. La segunda estrategia consiste en el re-secuenciamiento óptimo de clientes no visitados cada vez que un vehículo visita un nuevo nodo, es decir, el VRPSD se formula como un proceso de decisión markoviano (Secomandi y Margot, 2009). En Laporte et al. 
(2002) se plantea el VRPSD como un problema lineal entero-mixto estocástico bi-etapa, se propone un algoritmo de descomposición Branch and Bound a través de la técnica $L$-shaped. Para resolver el problema, se consideran demandas independientes e idénticamente distribuidas.

En esencia, los modelos de VRP básicos son completamente adversos al riesgo, por lo tanto, la investigación está abierta para realizar propuestas sobre modelos alternativos bajo incertidumbre (Zhang et al., 2019).

\subsubsection{Factores externos}

El transporte es esencial a lo largo de toda la cadena de suministro, tanto de bienes como de servicios. Hoy en día, es creciente el interés en soluciones sostenibles para las organizaciones desde el punto de vista económico como ambiental. Las actividades desarrolladas en la generación de riqueza también tienen consecuencias no deseables como, por ejemplo, el riesgo en el transporte de sustancias peligrosas: desechos industriales, desechos hospitalarios, sustancias radioactivas, basuras, entre otros. Se consideran dos aspectos que se han identificado de gran impacto en la operación del transporte de carga, a saber:

- Riesgos de seguridad: En el transporte de materiales peligrosos como desechos hospitalarios o nucleares; agentes químicos corrosivos, inflamables o gases nocivos lo más importante es prevenir los riesgos de quien los transporta como de los sitios geográficos por donde se transita. La posibilidad de un posible accidente está directamente relacionada con la ruta seleccionada. Esta situación obliga a que los modelos clásicos de VRP se amplíen y adapten para considerar esos aspectos de riesgo. En la literatura, se encuentran algunos enfoques, por ejemplo, analizan el transporte de cilindros de gas de forma que la exposición a la cual se somete la población sea la menor posible (Tarantilis y Kiranoudis, 2001).

En Yuan et al. (2017) se propone el problema de enrutamiento multidepósito para transporte de material peligroso el Hazardous Material Multi-depot Vehicle Routing Problem (HMDVRP), donde se considera la carga dinámica del vehículo y se resuelve a través de una heurística de dos etapas, considerando un escenario biobjetivo.

En Androutsopoulos y Zografos (2012) se consideran ventanas de tiempo y duración del recorrido dependiendo de las condiciones del tráfico, se resuelve el problema mediante una heurística considerando una función multiobjetivo.

Taslimi et al. (2020) identifican el problema periódico de enrutamiento capacitado dependiente de la carga -Periodic Load-Dependent Capacitated Vehicle Routing Problem (PLCVRP)-, que se identifica en los centros de salud y en las empresas que se dedican a la recolección de desechos médicos deben realizar itinerarios 
semanales. En este caso, se considera el riesgo laboral, relacionado con el almacenamiento temporal de residuos peligrosos en los centros de salud. El riesgo de transporte en cada arco depende del peso de los desechos médicos peligrosos en el vehículo cuando atraviesa ese arco. Se diseña un algoritmo heurístico basado en descomposición para resolver este problema. El PLCVRP revela que minimizar tanto el transporte como el riesgo ocupacional en la red puede ayudar a los tomadores de decisiones a desarrollar un mejor programa de enrutamiento en términos del riesgo impuesto por los desechos médicos peligrosos. Se debe considerar que los modelos de enrutamiento de agentes peligros pueden generalizarse a agentes externos indeseables como ruidos, perturbaciones y seguridad de los transeúntes (Grabenschweiger et al., 2018).

- Emisiones ambientales: Dentro de los costos externos asociados al transporte aparecen las siguientes categorías: (i) emisiones ambientales, las cuales incluyen los gases de efecto invernadero, Greenhouse Gases (GHG) y la polución del aire, (ii) el ruido, (iii) el uso de la tierra y (iv) los riesgos de seguridad, incluyendo los accidentes (Demir et al., 2014). En particular, la reducción de los gases de efecto invernadero ha sido una temática de interés para la comunidad internacional debido al incremento de las temperaturas globales. El principal recurso que se usa para el transporte corresponde a los combustibles fósiles; por tanto, cualquier avance que se haga en dirección a minimizar los GHG son bienvenidos en la comunidad académica, gubernamental y empresarial; de allí la mirada a las fuentes alternativas de energía para realizar el transporte de mercancías así como la revaluación de los sistemas de transporte de tracción humana como una opción interesante en situaciones donde las cargas son livianas y las condiciones de los centros urbanos lo permiten.

Este análisis es de relevancia debido a que la población mundial sigue en aumento y, por tanto, el movimiento de todo tipo de bienes y servicios es vital para el desarrollo económico y para el bienestar de la población. La carga se desplaza a través del planeta a través de diferentes medios de transporte. En esta sección solo se considera la carga transportada por vía terrestre. Donde se hace un análisis de la temática para justificar, la presentación de un modelo para el problema de enrutamiento el cual considera la minimización de $\mathrm{CO}_{2}$ dentro de los objetivos a optimizar. Con base en diferentes análisis, se ha concluido que las emisiones de $\mathrm{CO}_{2}$ están relacionadas directamente con el consumo de combustible (Kent y Mudford, 1979; Schingh et al., 2000; Romilly, 1999).

El consumo de combustible se ve afectado por diferentes factores relacionados con:

- El vehículo: Peso del vehículo en vacío, forma del vehículo, motor (tamaño y tipo), temperatura del motor, sistema de transmisión, tipo de combustible, viscosidad del combustible, otras características (mantenimiento, edad, accesorios, etc.). 
- El medio ambiente: Gradiente de la vía, tipo de pavimento, temperatura del ambiente, altitud, condiciones del viento, otras características (humedad, condiciones de la superficie).

- El tráfico: Velocidad, acelaración/desaceleración, congestión.

- El conductor: Agresividad del conductor, tiempos de espera, selección de engranajes.

- La operación: Tamaño de flota y mezcla, carga útil, kilómetros vacíos, número de paradas.

Considerando el objetivo clásico de minimización de costos en términos de distancia o tiempo muchas de las soluciones óptimas para el VRP pueden tener correlación con las emisiones contaminantes, y generalmente se miden en relación con los galones de combustible consumidos entre dos puntos de la ruta. Dichas soluciones tienen en cuenta la pendiente de la vía, el peso, la aceleración y la velocidad del vehículo. Una variedad de modelos de consumo de combustible y de métodos de solución han sido propuestos para resolver diversas variantes del VRP. (i) Con base en la carga del vehículos (Kara et al., 2007); (ii) dependiendo del tiempo (Jabali et al., 2012); (iii) considerando una flota de vehículos heterogénea (Kwon et al., 2013; Li et al., 2018); (iv) considerando la localización de los centros de distribución (Toro et al., 2017b).

Dentro de las métricas usadas para medir las emisiones de $\mathrm{CO}_{2}$ se consideran las siguientes:

- Computer programme to calculate emissions from road transportation (COPERT), corresponde a un organismo de la Comunidad Económica Europea para modelar las emisiones contaminantes (Kouridis et al., 2010). En este se tiene una base de datos donde se encuentran las emisiones de acuerdo con las características del vehículo tales como la tecnología de motor, la clase de vehículo y la velocidad. COPERT usa una función de regresión lineal para estimar el consumo de combustible, el cual está asociado con el peso del vehículo y la comunidad académica va actualizando el factor de acuerdo con los avances en las investigaciones.

- Methodology for calculating transportation emissions and energy consumption (MEET) corresponde a una publicación de la Comisión Europea, realizada por Andre et al. (1995) sobre factores de emisiones del transporte en carreteras describe la metodología denominada MEET, usada para calcular las emisiones y el consumo de energía e vehículos de carga pesada. MEET está basado en medidas de la vía y todos esos parámetros son resultado de experimentos realizados en escenarios reales. Los experimentos consideraron vehículos con diferentes tecnologías para diferentes clases de vehículos, tales como vehículos de menos de 3.5 toneladas, vehículos con pesos entre 3.5 y 7.5 toneladas, vehículos con pesos entre 7.5 y 16 toneladas, vehículos con pesos entre 16 y 32 toneladas y vehículos que pueden transportar cargas de más de 32 toneladas. Para vehículos de menos de 3.5 toneladas, las emisiones de $\mathrm{CO}_{2}$ son estimadas usando una 
función de regresión lineal en función de la velocidad. Para los vehículos de otro tipo se usa una función con base en la mercancía que se va descargando del vehículo, considerando un gradiente cero sobre la vía. Otros valores de la ecuación de trabajo son parámetros tomados de tablas; esos valores se refieren a condiciones estándar, calculada, con base en una velocidad promedio. Estudios posteriores han dado lugar a una nueva expresión que hace una corrección con base en el gradiente de la vía.

- A comprehesive modal emisión model (CMEM) fue desarrollado y presentado por Scora y Barth (2006) para vehículos de transporte pesado. Este fue desarrollado en un laboratorio de conducción observando 343 tipos de vehículos de carga liviana. El resultado es un modelo que consta de tres módulos: potencia del motor, velocidad del motor y tasa de combustible. Cada uno de ellos tiene asociada una ecuación en función de sus parámetros específicos como los coeficientes de fricción y la energía del motor.

La Tabla nro. 2 hace un breve resumen de los elementos que se pueden considerar en la medición de los efectos contaminantes, asociados con el consumo de combustible, las métricas que usan y las variantes del problema de enrutamiento a las que se les ha aplicado. Dentro de las variantes del problema, se mencionan:

- Polution Routing Problem (PRP): Problema de Enrutamiento Considerado Polución.

- Emissions Vehicle Routing Problem (EVRP): Problema de Enruteamiento Considerando Emisiones.

- Vehicle Routing Problem with Time Windows (VRPTW): Problema de Enrutamiento con Ventanas de Tiempo.

- Fuel Consumption Vehicle Routing Problem (FCVRP): Problema de Enrutamiento Considerando Consumo de Combustible.

- Vehicle Routing Problem with Backhauls and Time Windows (VRPBTW): Problema de Enrutamiento de Vehículos con Entregas y Recogidas y Considerando Ventanas de Tiempo.

- Green Capacitated Location-routing Problem Considering Environmental Impact (GCLRP): Problema de Localización y Enrutamiento Verde sin Retorno al Depósito.

- Green Open Location-routing Problem Considering Economic and Environmental Costs (GOCLRP): Problema Verde de Localización y Enrutamiento Considerando Impacto Ambiental sin Retorno al Depósito.

Figliozzi (2010) denomina el EVRP como una extensión de el VRPTW, las emisiones contaminantes generadas por el combustible se plantean con base en la velocidad y la distancia recorrida de la ruta. La cantidad de emisiones se miden en relación con las millas recorridas y los cambios de velocidad a lo largo de la trayectoria. 


\begin{tabular}{|c|c|c|c|c|c|c|c|c|c|}
\hline \multirow[t]{2}{*}{ Referencia } & \multicolumn{5}{|c|}{ Característica } & \multicolumn{3}{|c|}{ Forma de cálculo } & \multirow[t]{2}{*}{ Problema y estrategia de solución } \\
\hline & $\mathrm{E}$ & V & $\mathrm{C}$ & PV & TA & $\mathrm{R}$ & $\mathrm{T}$ & Ec & \\
\hline Figliozzi (2010) & & $\mathrm{x}$ & & & & & & MEET & VRPTW / Heurística \\
\hline Kuo (2010) & & $\mathrm{x}$ & $\mathrm{x}$ & & & & & $\mathrm{x}$ & Recocido simulado / TDVRP \\
\hline Suzuki (2011) & & $\mathrm{x}$ & $\mathrm{x}$ & $\mathrm{x}$ & & & & $\mathrm{x}$ & TSPTW compressed annealing \\
\hline $\begin{array}{l}\text { Bektaş y Laporte } \\
\text { (2011) }\end{array}$ & & $\mathrm{x}$ & $\mathrm{x}$ & & & & & CMEM & PLE/PRP/TDVRP/CVRP. CPLEX 12.1 \\
\hline Ubeda et al. (2011) & & & $\mathrm{x}$ & & & & $\mathrm{x}$ & & $\begin{array}{l}\text { Heurística de dos fases. VRPB - Vecino más } \\
\text { cercano. CVRP/ Heurística de Mole Jameson }\end{array}$ \\
\hline Jaramillo (2011) & & & $\mathrm{x}$ & & & & & $\mathrm{x}$ & PLE / CVRP / CPLEX 12.2 \\
\hline Demir et al. (2012) & & $\mathrm{x}$ & $\mathrm{x}$ & $\mathrm{x}$ & $\mathrm{x}$ & & & CMEM & ALNS / VRPTW \\
\hline Xiao et al. (2012) & & & $\mathrm{x}$ & & & $\mathrm{x}$ & & & Recocido simulado. PLEB / CVRP \\
\hline Jemai et al. (2012) & & & & & & & & & NSGA-II / CVRP \\
\hline $\begin{array}{lll}\begin{array}{l}\text { Kucukoglu et al. } \\
\text { (2013) }\end{array} & & \\
\end{array}$ & & & $\mathrm{x}$ & & $\mathrm{x}$ & $\mathrm{x}$ & & $\mathrm{x}$ & PLE / G-CVRP / GUROBI \\
\hline $\begin{array}{lll}\begin{array}{l}\text { Pradenas } \\
\text { (2013) }\end{array} & \text { et al. } \\
\end{array}$ & $\mathrm{x}$ & $\mathrm{x}$ & $\mathrm{x}$ & $\mathrm{x}$ & & & & CMEM & PLEM/VRPBTW / Scatter search \\
\hline Pan et al. (2013) & $\mathrm{x}$ & & $\mathrm{x}$ & $\mathrm{x}$ & & & & COPERT & PLEM \\
\hline Demir et al. (2014) & & & & & & & & CMEM & Estado del arte \\
\hline Lin et al. (2014) & & & & & & & & & Estado del arte \\
\hline Kwon et al. (2013) & & & & & $\mathrm{x}$ & & & $\mathrm{x}$ & HFVRP / Búsqueda tabú \\
\hline Qian y Eglese (2016) & & $\mathrm{x}$ & & & & & & COPERT & CVRP/Heurística \\
\hline Toro 0 et al. (2016) & & & & & & & & & Estado del arte \\
\hline Toro et al. (2017b) & $\mathrm{x}$ & & $\mathrm{x}$ & $\mathrm{x}$ & & & & $\mathrm{x}$ & GCLRP / Epsilon Constraint \\
\hline Toro et al. (2017a) & $\mathrm{x}$ & & $\mathrm{x}$ & $\mathrm{x}$ & & & & $\mathrm{x}$ & G-OCLRP / Epsilon Constraint \\
\hline Li et al. (2018) & & $\mathrm{x}$ & $\mathrm{x}$ & $\mathrm{x}$ & & & & $\mathrm{x}$ & E-HFFVRP/ Búsqueda tabú \\
\hline Bektaş et al. (2019) & & & & & & & & & Estado del arte \\
\hline
\end{tabular}

TABLA NRo. 2. Emisiones de $\mathrm{CO}_{2}$ en el VRP.

Elaboración propia.

En Xiao et al. (2012) se considera el CVRP, analizando su comportamiento con base en el consumo de combustible el cual es representado por una regresión lineal a través de datos de una fuente del Ministerio de Tierras, Infraestructura, Transporte y Turismo de Japón. La propuesta se representa mediante una ecuación lineal en función del peso del vehículo en vacío denominado $Q_{0}$ y el peso del vehículo con carga denominada $Q_{1}$. Los valores que se usan para representar la expresión son: FCR - para el vehículo cargado ${ }^{-}$,definido como $\rho^{*}$ y el FCR cuando el vehículo en vacío se define como $\rho_{0}$; además $y_{i j}$ representa la carga a ser transportada entre los nodos $i$ y $j$. Las ecuaciones que representan el consumo de combustibles son 1.7 y 1.8 :

$$
\begin{gathered}
\rho_{i j}=\rho_{0}+\frac{\rho^{*}-\rho_{0}}{Q} y_{i j}=\rho_{0} \alpha y_{i j} \\
C_{\text {combustible }}=\sum_{i=1}^{r} \sum_{j}^{r} C_{\text {combustible }}^{i j} x_{i j}=\sum_{i=1}^{r} c_{0} \rho_{i j} d_{i j} x_{i j}
\end{gathered}
$$

En Jemai et al. (2012), el GVRP es propuesto como una variante del CVRP, el cual es resuelto como un modelo bio-objetivo a través del algoritmo NSGA-II. Los operadores que utiliza son: para el crossover, el partially mapped crossover (PMX); para la mutación, se realizan intercambios de posición de los nodos en las rutas. La técnica para calcular las emisiones contaminantes es la propuesta en McKinnon y Woodburn (1996). 
Este documento presenta un modelo matemático flexible y una metodología metaheurística que puede ser adaptada a diferentes variantes del problema de enrutamiento las cuales se presentan en la Figura nro. 1. Este se basa en el problema clásico del agente viajero, denominado en inglés como travel salesman problem (TSP), donde se necesita definir una ruta que visite todos los nodos una única vez. En algunas aplicaciones no se permite que la longitud de ruta exceda un límite de tiempo o de distancia, lo que hace necesaria la confección de varias trayectorias o ciclos para visitar todo el conjunto de clientes, esto configura el problema de múltiples agentes viajeros, multitravel salesman problem (M-TSP). En ambos problemas, el objetivo es visitar todos los clientes, minimizando el costo de los arcos activos (Bolanos et al., 2016). Basado en el M-TSP, se define el problema de enrutamiento capacitado Capacitated Vehicle Routing Problem (CVRP), en donde se define uno de los nodos como depósito de mercancía con capacidad ilimitada desde el cual se atienden los clientes dispersos geográficamente, de ellos se conoce la localización y una cantidad de mercancía demandada. Para atenderlos, el recurso disponible es una flota de vehículos con capacidad homogénea. La función objetivo es entregar un conjunto de rutas cuya sumatoria sea lo menor posible cumpliendo con las siguientes restricciones: (i) Atencíón completa de la demanda de todos los clientes, (ii) no se debe exceder la capacidad de los vehículos disponibles; (iii) se tiene una flota limitada de vehículos, por tanto, la solución debe usar esa cantidad de vehículos o menos. En el evento que se requiera ser más eficiente en las entregas a los clientes finales minimizando, por ejemplo, los tiempos de entrega; se puede tomar la decisión de ubicar otros depósitos o centros de distribución que atiendan zonas específicas. Esta situación se configura cuando la operación aumenta 0 cuando se requiere mejorar en la calidad del servicio. Dado lo anterior, se configura el problema de enrutamiento con múltiples depósitos Multi-Depot Vehicle Routing Problem (MDVRP), en esta variante la localización de los depósitos se ha definido previamente y no es una decisión a tomar. Por el contrario, cuando se debe definir la ubicación de los depósitos y la capacidad de los mismos, se configura el problema de localización y ruteo capacitado Capacitated Location Routing Problem (CLRP). Debido a que se deben resolver varios problemas como (i) decidir qué depósitos se deben abrir de un conjunto de opciones disponibles, (ii) asignar los clientes al depósito, (iii) diseñar las rutas que atiendan completamente la demanda; la función objetivo corresponde a la minimización de un conjunto de costos así: apertura de depósitos, costos fijos por vehículo usado, sumatoria de las distancias o tiempos de recorrido de todas las rutas. En esta variante, la flota de vehículos es ilimitada.

El modelo matemático que se presenta en este documento puede ser adaptado, activando y desactivando variables y restricciones para cada variante del VRP, y es eficiente para encontrar solución a una amplia variedad de problemas como se ilustra en la Figura nro. 1 y cuyas siglas se definen a continuación:

\section{- Problemas de enrutamiento clásicos.}

- Travel Saleman Problem (TSP). 


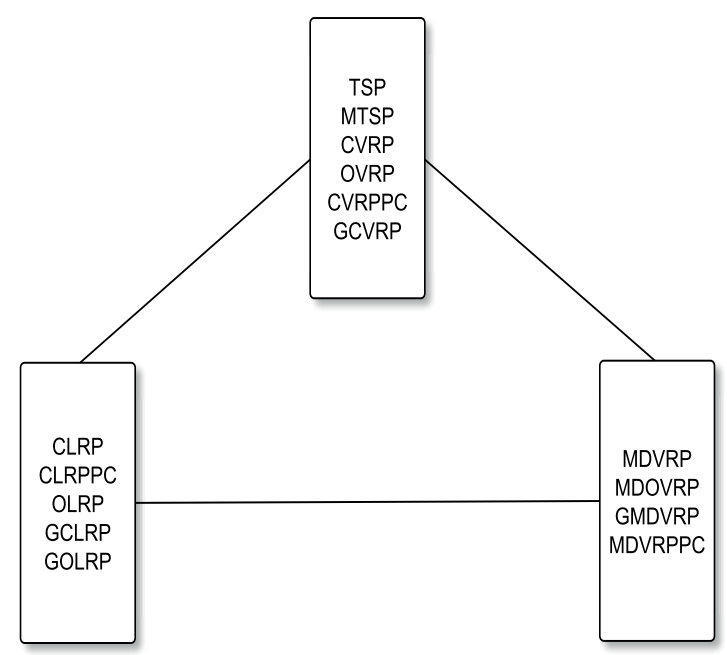

Figura NRO. 1. Tipos de VRP que pueden resolverse con el modelo matemático propuesto. Elaboración propia.

- Capacitated Vehicle Routing Problem (CVRP).

- Multi-Depot Vehicle Routing Problem (MDVRP).

- Capacitated Location Routing Problem (CLRP).

- Problemas abiertos de enrutamiento sin retorno al depósito.

- Open Vehicle Routing Problem (OVRP).

- Multi-Depot Open Vehicle Routing Problem (MDOVRP).

- Open Capacitated Location Routing Problem (OLRP).

- Problemas de enrutamiento considerando flota propia y subcontratada

- Vehicle Routing Problem With Private Fleet And Common Carrier (VRPPC).

- Multi-Depot Vehicle Routing Problem With Private Fleet And Common Carrier (MDVRPPC).

- Capacitated Location Routing Problem With Private Fleet And Common Carrier (CLRPPC).

- Problemas de enrutamiento considerando efectos ambientales.

- Green Capacitated Vehicle Routing Problem (GVRP). 
Eliana Mirledy Toro Ocampo, John Fredy Castañeda Londoño, Ramón Alfonso Gallego Rendón

- Green Multi-Depot Vehicle Routing Problem (G-MDVRP).

- Green Capacitated Location-Routing Problem Considering Environmental Impact (G-CLRP).

- Green Open Location-Routing Problem Considering Economic and Environmental Costs (G-OLRP). 


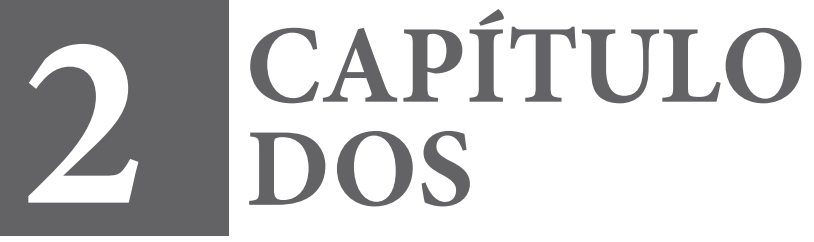




\section{TÉCNICAS DE SOLUCIÓN PARA RESOLVER LOS VRP}

En el estudio de las técnicas de solución para los problemas de enrutamiento se considera que a aquella que sea eficiente para el CVRP puede ser eficiente para cualquier otra variante, debido a que este es el problema básico de la cadena de distribución. En el desarrollo de las metodologías de solución para abordar los problemas combinatorios se van surtiendo etapas; inicialmente, se presentan modelos matemáticos que tienen la capacidad de resolver problemas de pequeño porte pero, en la medida en que se refinan los modelos, se pueden diseñar metodologías aproximadas capaces de resolver instancias de tamaño medio. No se puede desconocer que, antes de la aparición formal de un modelo matemático, la situación es resuelta con base en el conocimiento del problema por quien gestiona con este diariamente. Aquí surgen las metodologías heurísticas que pueden ser potenciadas con estrategias inteligentes con el fin de desarrollar la búsqueda en el espacio de soluciones para instancias de mayor tamaño y generalmente en tiempos computacionales más cortos. En los años 90, aparecen las metaheurísticas (Gallego-Rendón et al., 2015) como estrategia de solución que, basadas en el comportamiento de la naturaleza, copian su comportamiento para gestionar la búsqueda de regiones promisorias con el fin de desarrollar soluciones de buena calidad. Finalmente, en la última década se han propuesto técnicas híbridas como las math-heurísticas cuya filosofía es dividir el problema en etapas: una de ellas se resuelve de forma exacta y otra(s) de forma aproximada mediante el uso de las heurísticas y metaheurísticas. Al respecto, la Figura nro. 2 presenta este resumen de estrategias. 


\subsection{Problema de enrutamiento de vehículos considerando restricciones de capacidad (CVRP)}

El CVRP puede definirse como un grafo completo donde todos los nodos se comunican entre sí. Este consiste en un conjunto de clientes de los que se conoce su localización geográfica y su demanda, se tiene un depósito quien se incluye como un nodo con capacidad ilimitada de mercancía para atender la sumatoria de demanda de todos los clientes y se cuenta con una flota limitada de vehículos con capacidad limitada de carga. El objetivo es definir un conjunto de rutas que atienda a todos los clientes en una única visita sin exceder las restricciones de capacidad del vehículo asignado a la ruta.

\subsubsection{Técnicas exactas para resolver el CVRP}

Dentro de los métodos exactos se pueden enumerar los siguientes trabajos: $i$ ) Modelo exacto usando poliedros (Araque et al., 1990), ii) En Fischetti et al. (1994) se presenta una adaptación del algoritmo Branch and Bound al CVRP, iii) En Naddef y Rinaldi (2002) se propone un conjunto de desigualdades válidas y se resuelve el problema usando Branch and Bound, iv) Baldacci et al. (2011b) presenta el algoritmo Branch and Price con una estrategia de generación de columnas que demarcan rutas para mejorar la calidad de la función objetivo. v) En Laporte (1992), Toth y Vigo (2002) y Golden et al. (2008) se tiene un compendio de métodos y modelos exactos para resolver el problema.

\subsubsection{Métodos heurísticos para resolver el CVRP}

Las estrategias que son eficientes para resolver el TSP, en su gran mayoría, son eficientes para resolver el CVRP debido que se sustentan en la misma filosofía de atender un conjunto de clientes en el menor tiempo o gastando la menor distancia posible. En la implementación de la heurística, la codificación generalmente es un vector que representa el orden de la visita; para el caso del TSP, se representa con un único vector, pero para el CVRP se tendrán tantos vectores como rutas sean necesarias para atender todos los clientes. Estas técnicas se clasifican en:

- i) Técnicas constructivas. Estas indican que se va construyendo cada ruta paso a paso verificando la factibilidad de la respuesta, básicamente la factibilidad se garantiza clasificando los clientes o nodos en dos grupos (uno de clientes visitados y otro de clientes no visitados), las rutas se van construyendo con los clientes no visitados. Al final se valida la calidad de la respuesta realizando la suma de los arcos activos. Dentro de este grupo la heurística de ahorros es una de las más eficientes. Esta ha sido ampliamente estudiada por la comunidad académica y generalmente se adapta a las diferentes variantes del VRP. Algunos estudios detallados pueden ser consultados 
en Clarke y Wright (1964), Hinson y Mulherkar (1975), Norback y Love (1977), Golden et al. (1980) y Golden y Wong (1981). Otro tipo de heurística constructiva muy estudiada corresponde a las de inserción (Potvin y Rousseau, 1990; Laporte y Semet, 2002). Las inserciones deben ser factibles para ser permitidas (Bodin, 1975). Sin embargo, este concepto ha sido reevaluado porque se han realizado análisis donde las inserciones infactibles llevan a procedimientos de reparación que pueden generar soluciones de mejor calidad.

- ii) Heurísticas de dos fases: Estas indican que para entregar una respuesta factible se divide el proceso en dos etapas, para el CVRP se tienen dos filosofías. La primera consiste en asignar primero y rutear después, y dividir el conjunto de clientes en subconjuntos. El criterio del agrupamiento o clusterización es la capacidad del vehículo que atenderá la ruta. El número de subconjuntos de clientes no debe exceder la cantidad de vehículos disponibles; luego, cada subconjunto es resuelto como un TSP para determinar el orden de visita de los clientes. Estrategias de este tipo aparecen descritas en Wren y Holliday (1972), Gillett y Miller (1974) y Karp (1977). La otra forma de resolver el problema es enrutar primero y asignar después. Esta estrategia consiste en encontrar un TSP con todos los nodos, sin considerar la demanda de los clientes. De esta forma, se obtiene un tour gigante que se empieza a dividir desde algún punto que se elija y se van agregando los nodos adyacentes hasta copar la capacidad del vehículo. Cuando ese criterio se cumple, se inicia la construcción de la siguiente ruta a partir del siguiente cliente que no se ha servido. Este tipo de estrategia ha sido discutida por Beasley (1983).

iii) Heurísticas de mejora iterativa: Este tipo de estrategias requieren de una solución de inicio, obtenida por una de las técnicas anteriores o a través de un procedimiento aleatorio. Consiste básicamente en un intercambio de nodos en la secuencia de la ruta o en el levantamiento de arcos que obligan a que se reconecten con el resto de la ruta. Se cuenta con un gran número de estrategias que han sido suficientemente documentadas en la literatura, dentro de ellas se tiene: «Lambda intercambio» (Lin, 1965), «GENI y GENIUS» (Gendreau et al., 1992), «transferencias ciclícas» (Thompson y Psaraftis, 1993), «vecindario cruzar-intercambiar» (Taillard, 1993), «operadores de Van Breedam» (Breedam, 1995) y «Or-OPT» (Azi et al., 2009). La más eficiente de estas estrategias es la de Lin-Kernighan que consiste en intercambios sucesivos de arcos mediante la estrategia « Or-OPT» (Helsgaun, 2000, 2009; Karapetyan y Gutin, 2011; Helsgaun, 2015).

\subsubsection{Adaptación de técnicas metaheurísticas para resolver el CVRP}

Las técnicas metaheurísticas se han constituido en una estrategia exitosa para resolver problemas combinatoriales; en Gallego-Rendón et al. (2015) se hace una amplia discusión de la implementación de diferentes heurísticas y metaheurísticas para resolver problemas NP y NP-duros. Además, se indica la necesidad de tener una codificación eficiente que permita realizar una exploración del espacio de soluciones con resultados exitosos. Es claro que estas 
estrategias requieren soluciones de inicio de buena calidad, generalmente, obtenidas mediante heurísticas eficientes o criterios de sensibilidad basados en la naturaleza del problema a resolver.

El VRP es un problema NP-duro, por lo tanto, las metaheurísticas se constituyen en una herramienta de relevancia para encontrar soluciones en tiempos computacionales cortos debido a que se está resolviendo un problema operativo. Un estudio taxonómico publicado entre el 2009 y 2015 afirma que alrededor del $71.25 \%$ de los artículos estudiados utilizan estas técnicas para resolver los VRP (Braekers et al., 2016). En Elshaer y Awad (2020) y Blocho (2020) se realizan actualizaciones de estas técnicas de solución, aplicadas a diferentes variantes del VRP.

El algoritmo genético es una de las metaheurísticas más estudiadas para resolver todo tipo de problemas combinatoriales y el VRP no es la excepción. Esta técnica ha sido adaptada por Pereira et al. (2002), Baker y Ayechew (2002), y Nazif y Lee (2012) donde se presenta un procedimiento de recombinación mejorado, adicionalmente en Osaba et al. (2013) se hace una discusión del método. El algoritmo «colonia de hormigas» ha sido adaptado por Ai y Kachitvichyanukul (2009) y Yu et al. (2009).

Respecto a los métodos híbridos, en Marinakis y Marinaki (2010) se combinan estrategias de colonia de hormigas y algoritmo genético. Por su parte, en Wang y Lu (2009) incorporan estrategias de barrido en la recombinación de un genético definido en tres etapas.

El algoritmo de «búsqueda tabú» es una de las técnicas metaheurísticas de mejor adaptación para resolver el problema y se evidencia en Osman (1991), Taillard (1993), Toth y Vigo (2003), Nuortio et al. (2006), Zachariadis y Kiranoudis (2012), Cordeau y Maischberger (2012), Janssens et al. (2015), Jin et al. (2014), Seyyedhasani y Dvorak (2017) y Lin et al. (2009).

\subsection{Problema de enrutamiento considerando múltiples depósitos (MDVRP)}

El MDVRP puede ser formulado como un grafo completo y dirigido donde se tienen dos conjuntos de nodos, uno de los conjuntos representa los depósitos desde donde se realizan los despachos de mercancía a los clientes. Cada depósito tiene una capacidad limitada. Además, se tiene un conjunto de clientes de los que se conoce su localización y su demanda. Los clientes deben ser atendidos una única vez por alguna ruta que inicie en un depósito. La solución se entrega como un conjunto de rutas entre los depósitos y los clientes. Cada ruta tiene asociado un costo que generalmente es representado como la longitud de la ruta. El objetivo es minimizar la sumatoria de los costos de las rutas.

Dentro de la literatura aparecen reportadas las siguientes aplicaciones: Distribución de alimentos procesados 
(Cassidy y Bennett, 1972), reparto de gases industriales (Bell et al., 1983), distribución de petróleo (Brown et al., 1987), en Golden y Wasil (1987) se reporta la entrega de pedidos de bebidas gaseosa, reparto de lácteos (Pooley, 1994) y entrega de productos químicos (Ball et al., 1983).

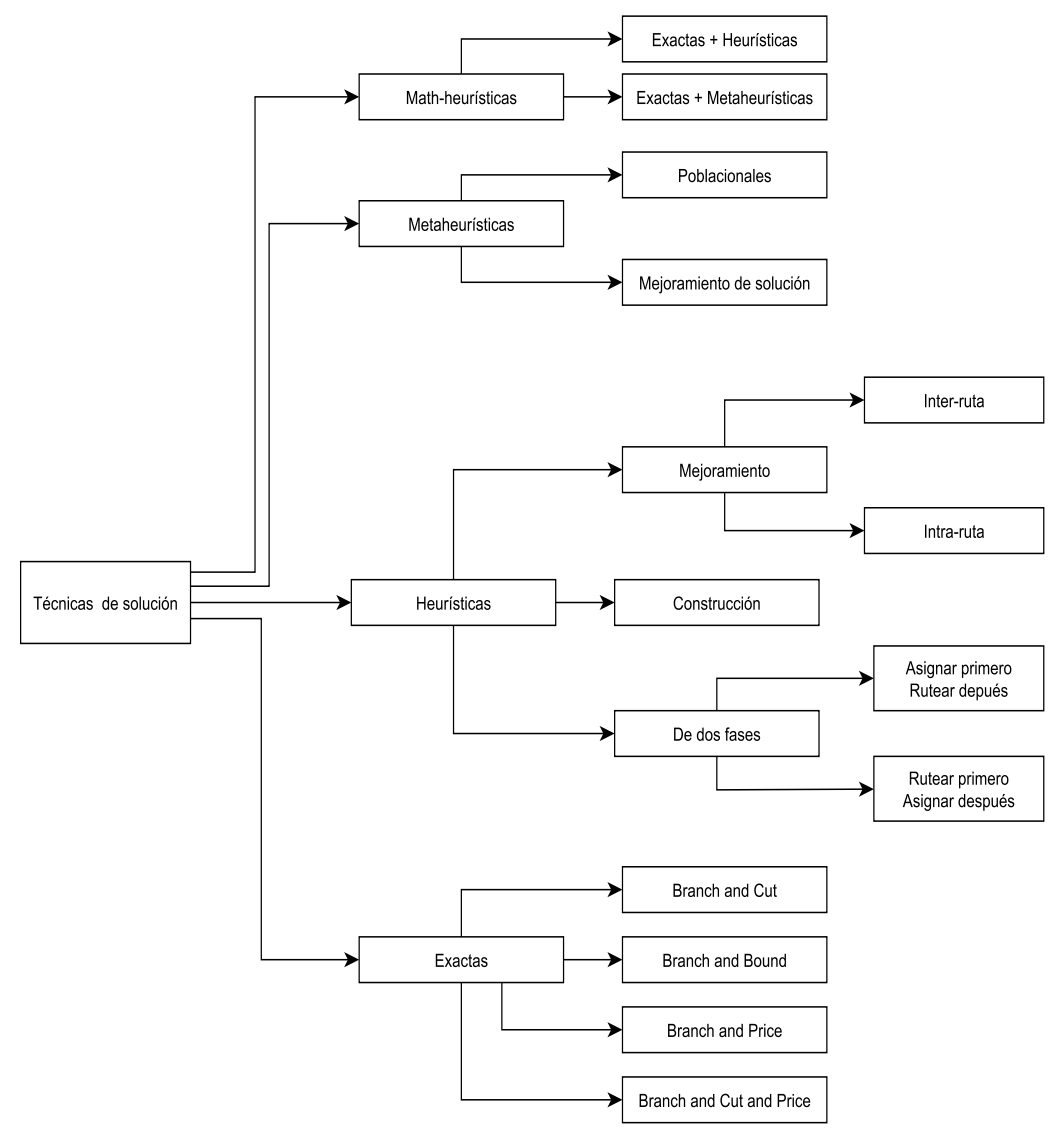

Figura NRo. 2. Métodos de solución para los problemas de Enrutamiento. Elaboración propia.

\subsubsection{Técnicas exactas para resolver el MDVRP}

En Baldacci y Mingozzi (2009) se definen límites inferiores usando relajación lineal y lagrangiana con base en Christofides et al. (1981). Esta es una estrategia que reduce el número de variables del modelo. El MDVRP con 
visitas periódicas se define en Baldacci et al. (2011a) y se resuelve usando un método y exacto, eficiente definiendo previamente límites inferiores para el problema. En Contardo y Martinelli (2014) se divide el problema en dos etapas, cada una de las cuales es resuelta mediante un método exacto, robustecido, con la propuesta de un conjunto de nuevas restricciones válidas que mejoran la exploración del espacio de soluciones.

\subsubsection{Técnicas heurísticas para resolver el MDVRP}

El clásico algoritmo de ahorros propuesto en Clarke y Wright (1964) es adaptado en Tillman y Cain (1972). Ellos emplean una métrica de distancia, considerando la naturaleza del problema. En Salhi y Sari (1997), se desarrolla una propuesta de varios niveles. Se requiere una solución de inicio hallada en la primera etapa; luego, los niveles posteriores funcionan como mecanismos de mejoramiento usando heurísticas inter- e intra- depósito. La estrategia de agrupar primero y rutear después es propuesta en Giosa et al. (2002) y es aplicada al problema de enrutamiento considerando ventanas de tiempo (MDVRPTW). La metodología tiene dos etapas: en la primera se hace la asignación de los clientes a los depósitos y, en la segunda, se aplica el algoritmo de ahorros a cada clúster, y se prueban y comparan diferentes técnicas de agrupamiento. El MDVRP, considerando entregas y recogidas simultáneas, Multi-Depot VRP with Mixed Pickup and Delivery (MDVRPMPD), es resuelto en Nagy y Salhi (2005), la metodología propuesta consta de cuatro etapas: i) Categorizar los clientes cercanos y no cercanos; i) los clientes no cercanos se asignan al depósito más cercano a ellos; iii) para cada depósito, se resuelve un problema de entregas y recogidas Vehicle Routing Problem with Pickups and Deliveries (VRPPD); finalmente, $i v)$ se adicionan los clientes no asignados más cercanos a cada clúster, posteriormente se realiza una etapa de mejoramiento usando 2-OPT y 3-OPT, reinserciones e intercambios.

En Lee et al. (2006) se plantea el problema usando programación dinámica determinista con estados finitos. Esto es resuelto con una versión heurística de un algoritmo de ruta más corta.

Una heurística paralela con conceptos de «búsqueda tabú» se propone en Cordeau y Maischberger (2012) se usa un mecanismo de perturbación para explorar el espacio de soluciones. Esta estrategia se potencia usando procesamiento paralelo.

\subsubsection{Técnicas metaheurísticas para resolver el MDVRP}

En Crevier et al. (2007) se propone una técnica híbrida entre «búsqueda tabú» y programación lineal entera, los vehículos pueden reaprovisionarse en depósitos intermedios que se han ubicado previamente en la ruta. Jeon et al. (2007) propone un algoritmo genético híbrido donde la población inicial es mejorada mediante heurísticas y se usa una tasa de mutación flotante para no quedar atrapado en óptimos locales.

En Ho et al. (2008) se proponen dos algoritmos genéticos híbridos (HGA1 y HGA2). En el HGA1, se genera 
la población inicial de forma aleatoria y en el HGA2 se usan el algoritmo de ahorros y la heurística del vecino más cercano para generar las soluciones de inicio. La propuesta es validada con dos casos propios donde se consideran dos depósitos con 50 y 100 clientes.

En Surekha y Sumathi (2011) se aplica el algoritmo genético. Para generar las soluciones iniciales, se usan técnicas de agrupamiento basadas en la distancia a los depósitos más cercanos y luego se diseñan las rutas usando la heurística de ahorros.

Un algoritmo genético e híbrido que permite soluciones infactibles es propuesto en Vidal et al. (2012). Se divide la población en dos conjuntos basados en la categoria de la solución: factibles e infactibles. De cada conjunto se eligen dos individuos padres y se combinan para generar un nuevo individuo que es mejorado mediante estrategias de búsqueda local. Después, se calcula la factibilidad y con base en ella se categoriza para ubicarlas en la subpoblación apropiada. Con esta metodología se alcanzan todos los óptimos de la literatura.

Un algoritmo «búsqueda tabú granular híbrido» se propone en Escobar et al. (2014). Estos autores utilizan conceptos de vecindarios y de diversificación en una etapa de mejoramiento posterior a la generación de la solución inicial, hallada a través de un procedimiento híbrido inicial. Los resultados son competitivos para todo el conjunto de prueba en cuanto a la calidad de las respuestas y los tiempos computacionales.

En Subramanian (2012) se plantea una técnica math-heurística que combina una secuencia de modelos lineales enteros mixtos con una estrategia de generación de columnas (rutas), las cuales se obtienen por una aproximación metaheurística. Esta metodología general puede resolver de forma eficiente un conjunto de variantes del VRP.

Un estado del arte es presentado en Montoya-Torres et al. (2015). Se relacionan 173 publicaciones disponibles desde 1988, se presentan variantes del problema y diferentes técnicas de solución para escenarios mono y multiobjetivo. Un resumen de diferentes estrategias que tienen como base el algoritmo genético se presenta en Karakatič y Podgorelec (2015). En este texto hace una discusión de diferentes aproximaciones, adaptaciones de los operadores y aplicaciones prácticas.

En Li et al. (2016b) se desarrolla una nueva variante para el MDVRP bajo las limitaciones de las ventanas del tiempo, la capacidad y la duración de la ruta del vehículo, el tamaño de la flota y el número de plazas de estacionamiento de cada depósito. En este escenario, se plantea una metodología que usa un algoritmo genético híbrido con búsqueda local adaptativa.

En Bae y Moon (2016) se demuestra que los MDVRPTW se pueden utilizar para minimizar los costos fijos de los depósitos, los costos fijos de los vehículos de entrega y los gastos relacionados con las distancias de viaje, así como la mano de obra, por medio de un algoritmo genético híbrido. Los resultados son promisorios pues se resuelven 
instancias de hasta 100 clientes y 4 depósitos.

En Ocampo et al. (2016) se propone una metodología híbrida entre técnicas de agrupación propuestas por Barreto et al. (2007). Estas se usan como inicializador en la primera etapa; en la segunda, se aplica el algoritmo Iterated Location Search (ILS) para explorar el espacio de soluciones. Luego, se realiza un análisis del efecto que tiene la técnica de inicialización en el desempeño del algoritmo. La propuesta se valida con casos de la literatura donde se observa un buen desempeño.

En el trabajo desarrollado por Du et al. (2017) se presenta un modelo de programación difuso de dos niveles para el MDVRP el cual pretende minimizar el riesgo de transporte cuando se entregan productos de materiales peligrosos. El nivel superior se asigna a las limitaciones de las capacidades de los depósitos y a las demandas de los clientes, mientras que en el nivel inferior se determina la ruta óptima.

En Alinaghian y Shokouhi (2018) se desarrolla un modelo matemático donde se minimiza el número de vehículos y el total de rutas recorridas mediante un algoritmo híbrido, compuesto por la búsqueda adaptativa de grandes vecindarios y el ILS para resolver instancias de gran escala.

En Ramos et al. (2020) se proponen las restricciones de: Dantzig-Fulkerson-Johnson, Miller-Tucker-Zemlin, de la carga de tránsito y del tiempo de llegada para eliminar los sub-tours, con una formulación de flujo de dos productos, considerando una flota de vehículos heterogénea y un tiempo máximo de recorrido.

\subsection{Problema de localización de instalaciones con restricciones de capacidad (CFLP)}

Uno de los problemas de planificación de mayor relevancia en la logística de distribución corresponde al CFLP gracias a que generalmente se deben realizar inversiones altas en la adquisición de terrenos y en la construcción y adaptación de las instalaciones; por lo tanto, este ha recibido gran atención por parte de todo tipo de organizaciones gubernamentales, económicas y sociales. Con frecuencia, se debe decidir dónde abrir sucursales o puntos de distribución para mejorar la calidad del servicio en negocios como restaurantes, droguerías, supermercados, gasolineras, centrales de transmisión y distribución de todo tipo de servicios públicos. Así mismo, se deben tomar decisiones sobre la ubicación de instalaciones para la atención de comunidades como: estaciones de policía, bomberos, hospitales, escuelas, centros de acopio de productos perecederos, etc. En este tipo de decisión influyen elementos como el objetivo que se busca, el número de instalaciones a ubicar y el espacio de soluciones a considerar. Este último puede definirse discreto si se tienen $n$ ubicaciones candidatas a ser elegidas o puede ser continua si la instalación se ubica en cualquier punto de una región continua. Este se configura en Ghiani et al. (1999) donde se introduce el problema 
de localización de múltiples plantas que pueden ser abierta en el mismo sitio, capacitated plant location problem with multiple facilities (CPLPM).

El CFLP es un problema combinatorial donde de $n$ alternativas de ubicación se deben elegir $k$ opciones con criterios como minimización de costos o maximización del impacto en una comunidad. El paso seguido consiste en asignar los clientes a las locaciones que se deciden instalar, cumpliendo con varias restricciones: $i$ ) La demanda de cada cliente debe ser completamente satisfecha, ii) se asigna un costo de apertura a cada instalación, iii) se debe atender la demanda de todos los clientes (Ulukan y Demircioglu, 2015).

\subsubsection{Técnicas heurísticas para resolver el CFLP}

En Jacobsen (1983) se realiza una generalización de heurísticas para resolver el problema. Este autor considera el método de sustitución de vértices, Vertex Substitution Method (VSM), y heurísticas de adición. En Domschke y Drexl (1985) se presentan diferentes procedimientos para plantear soluciones de inicio y luego se le aplican heurísticas de adición para mejorar la calidad de la respuesta. Una solución heurística que emplea conceptos de «búsqueda tabú» con incorporación de oscilación estratégica, memorias de corto y largo plazo se presenta en Rolland et al. (1996).

\subsubsection{Técnicas metaheurísticas para resolver el CFLP}

En Bornstein y Azlan (1998) se propone el algoritmo de recocido simulado; por su parte, un algoritmo genético híbrido combinado con una técnica de subgradiente se propone en Doong et al. (2007). En Rahmani y MirHassani (2014) se presenta una modificación del algoritmo genético adaptando el fenómeno de bioluminiscencia empleado por las luciérnagas para mejorar la comunicación entre la población de individuos.

En Harris et al. (2014) se propone un algoritmo evolucionario y multiobjetivo considerando costos financieros y ambientales con resultados de buena calidad para casos de gran tamaño. La técnica simplificada de enjambres se propone como estrategia de solución en Lai et al. (2019), en una aproximación multiobjetivo usando una técnica de clasificación no dominada para generar el Pareto óptimo.

En Ozgen y Gulsun (2014) se desarolla una combinación de programación lineal y el análisis jerárquico fuzzy se propone como técnica multiobjetivo considerando la minimización de costos de apertura contra la maximización de beneficios de factores cualitativos.

Una metodología híbrida se propone en Li et al. (2014) considerando plantas con capacidad limitada donde se producen múltiples productos. En Gimadi et al. (2019) se expone una técnica híbrida que combina Programación de Memoria Adaptativa de Relajación (Relaxation Adaptive Memory Programming) con relaciones primales-duales y la búsqueda de subgrupos de Lagrange. 


\subsection{Problema de localización y enrutamiento (CLRP)}

Dentro del gerenciamiento de cadena de suministros, la logística de distribución de última milla es tal vez el mayor factor de interés dado que representa el eslabón final entre empresas y usuarios. Durante las últimas dos décadas, se han realizado estudios promisorios, enfocados a medir la satisfacción de los clientes debido a que la logística de distribución de última milla constituye el factor de mayor complejidad y relevancia. Muchos de estos estudios poseen gran variedad de aplicaciones en los diferentes campos de la ingeniería y corresponden a problemas de enrutamiento tradicionales.

\subsubsection{Técnicas exactas para resolver el CLRP}

Considerando las restricciones de demanda las técnicas exactas, basadas en relajaciones lagrangianas, se han propuesto por varios autores, entre ellos: Nauss (1978), Geoffrion y McBride (1978), Christofides y Beasley (1983), Pirkul (1987), Shetty (1990) y Alenezy (2020). Adaptaciones del algoritmo Branch and Bound, se presentan en Nauss (1978). Una adaptación de la descomposición de Benders se aplica a un modelo de Programación Lineal Entera Mixta (PLEM) en Golden y Wong (1981). Posteriormente, Van Roy (1986) combina la descomposición de Benders con la relajación lagrangiana. De forma sucesiva, este autor resuelve el problema de transporte y la ubicación de cada instalación.

El análisis poliedral del problema ha sido analizado en Leung y Magnanti (1989) y Aardal et al. (1995) con el fin de identificar cortes válidos que permita mejorar el desempeño de las técnicas exactas.

Por un lado, Lorena y Senne (2004) llevan a cabo una generación de columnas en un algoritmo Branch and Price para la solución del problema de la P-mediana. Por su parte, Klose y Gortz (2007) proponen una estrategia de generación de columnas y lo incorpora a un algoritmo de Branch and Price.

Por otro lado, Berger et al. (2007) adapta un algoritmo Branch and Price: cada columna que se agrega al proceso es una ruta. Lo anterior, va de la mano con el problema de ruta más corta, propuesto por Feillet et al. (2004), al considerar solo una restricción de longitud de la ruta. La ramificación se aplica, inicialmente, a las variables binarias de localización y, posteriormente, a las variables de rutas, usando la metodología propuesta en Ryan y Foster (1981).

En Akca et al. (2009) se propone una heurística basada en el Branch and Price y se proponen tres métodos exactos, potenciados con cinco nuevos cortes válidos que vuelven el espacio de soluciones polinomial. El pricing se resuelve usando el algoritmo de la ruta más corta con restricción de recursos, Elementary Shortest Path Problem with Resource Constraints (ESPPRC).

Una formulación de PLEM de dos índices usando dos tipos de variables binarias es propuesto en Belenguer 
et al. (2011). Las variables binarias se asocian al uso de los arcos, a la duplicidad de los arcos y a la apertura de los depósitos. En esa dirección, se propone un conjunto de cortes válidos, tomados de propuestas para el CVRP, con el fin de que se separen los clientes y los depósitos en subgrupos.

En Baldacci et al. (2004) se proponen estrategias de división para el CLRP, de esa forma se obtienen subproblemas MDVRP. Las características del método consisten en la reducción del número de subconjuntos de depósitos, los MDVRP son de menor complejidad computacional que el CLRP.

La metodología consta de tres fases: i) Se encuentran límites inferiores para el LRP usando el Source Capacitated Facility Location Problem (SSCFLP) o con el problema de múltiple elección tipo mochila. ii) Se aplica el Branch and Bound para todos los subconjuntos obtenidos en la etapa anterior. Este es planteado como un MDVRP. iii) Se resuelve de forma iterativa el CLRP considerando la minimización de la sumatoria de costos de apertura. En Contardo et al. (2013) se extiende el trabajo de Belenguer et al. (2011) y se proponen nuevas formulaciones basadas en flujos considerando dos y tres índices. Las formulaciones de tres índices tienen mejor desempeño que las de dos índices, aunque su tiempo de convergencia es mayor.

En Contardo et al. (2014a) se adiciona, al modelo de Baldacci et al. (2004), el conjunto de cortes planteados en Contardo et al. (2013) y se integran las variables-ruta, propuestas en Akca et al. (2009). El algoritmo consta de tres fases: $i$ ) Se resuelven los modelos de dos índices con base en la propuesta de Belenguer et al. (2011) y se usa un algoritmo de Branch and Cut relajando todas las variables a excepción de las binarias de la apertura de ruta. A cada subconjunto de depósitos se le aplica la siguiente etapa, a saber: ii) Cada MCDVRP se resuelve usando cortes y generado columnas. iii) Se determina una solución entera para cada MCDVRP.

En Aardal et al. (2015) se propone una aproximación polinomial para cada cliente el cual se representa como un problema de transporte, con esta estrategia se puede resolver el problema en tiempo polinomial fijándose el número de clientes en cada etapa.

Ponboon et al. (2016) propone un algoritmo Branch and Price para resolver el CLRP con ventanas de tiempo. El problema maestro se resuelve mediante un simplex y los sub-problemas elementales de ruta más corta con restricción de recursos se resuelven mediante el proceso de generación de columnas, el cual termina hasta que solo quedan las columnas de costo reducido no negativo. Se comprueba que el algoritmo es efectivo para instancias de gran tamaño. Por otro lado, Peng et al. (2017) logran una mejora significativa en el PSO, propuesto por Marinakis (2015), se incluyen nuevos esquemas de codificación para representar la solución del CLRP, y se emplean varios métodos de búsqueda local que permiten mejorar la calidad de cada solución. Para comprobar el funcionamiento del PSO, los autores evalúan algunos casos de prueba de la literatura, clásicos del CLRP.

Finalmente, en Ocampo et al. (2017) se propone un modelo matemático que aprovecha las condiciones de 
topología de la red de distribución y se plantea una alternativa eficiente que reemplaza las restricciones de eliminación de sub-tours la cual es validada para casos de la literatura con resultados satisfactorios.

\subsubsection{Técnicas metaheurísticas para resolver el CLRP}

Una metodología que combina el «recocido simulado» para seleccionar los depósitos, y el algoritmo de «colonia de hormigas» para determinar las rutas, es propuesto en Bouhafs et al. (2006). La calidad de las respuestas es comparable con las obtenidas en Barreto et al. (2007).

El GRASP mejorado con encadenamiento de trayectorias - Path Relinking (PR) - es propuesto en Prins et al. (2006). La metodología se implementa en varias etapas así: $i$ ) Se genera una solución de inicio usando el algoritmo de Clarke y Wright aleatorio aumentado, -Randomized Extended Clarke and Wright Algorithm (RECWA)-, respetando las restricciones de capacidad del vehículo y el depósito; posteriormente, se complementa con una fase de diversificación. ii) Si se obtienen rutas de cliente único y se fusionan aplicando el algoritmo de ahorros Clarke y Wright (1964); si no es posible la fusión por las restricciones de capacidad se finaliza esta etapa. iii) Se ejecuta una etapa de mejoramiento, aplicando intercambios y movimientos 2-OPT, la cual finaliza en el momento en que no se encuentren mejoras. $i v$ ) Finalmente, se lleva a cabo una etapa de post-optimización; aqui se generan nuevas soluciones desde el conjunto de soluciones élite. Se valida la calidad de la metodología aplicándose a casos TB, B y PPW.

En Prodhon y Prins (2008) se propone un Memetic Algorithm with Population Management (MA/PM) con las siguientes características: i) La codificación corresponde a un tour gigante sin restringir el número de rutas por depósito; el estado del depósito puede ser observado en una porción del cromosoma mediante una asignación binaria. ii) La población inicial se genera usando el RECWA y mediante el método del vecino más cercano aleatorizado, mejoradas a través de una búsqueda local. La población se refresca permanentemente conservando los mejores individuos, obtenidos hasta el momento, y se divide en dos subconjuntos (élite y no élite). iii)La estrategia de torneo es aplicada en el mecanismo de selección y se elige un padre de cada subconjunto de la población. iv) La recombinación se aplica tanto a la sección del depósito de forma clásica y a la sección de rutas se le aplica una recombinación tipo PMX. Se elije solo un descendiente y se usan criterios de aspiración para ingresarlo a la población actual. No se permiten configuraciones iguales dentro de la población y se utilizan medidas de distancia tomadas de Prins et al. (2006). La metodología se valida usando los casos de TB, B y PPW.

En Pirkwieser y Raidl (2010) resuelve el problema periódico de CLRP (PLRP) mediante una adaptación del algoritmo Variable Neighborhood Search (VNS) con un método exacto que resuelve de forma sucesiva PLE.

$\mathrm{El}$ «recocido simulado controlando la diversificación» es propuesto en Yu et al. (2009) para evitar quedar en óptimos locales, utiliza ubicación-asignación para decidir simultáneamente la localización y el enrutamiento. Los 
resultados se validan con instancias propuestas en Prins et al. (2007) y Duhamel et al. (2010). El algoritmo Adaptative Large Neighborhood Search (ILS) se propone en Prodhon (2011), gracias a este los vecindarios se evalúan y clasifican, luego se eligen para guiar la búsqueda a través de ellos.

En Marinakis y Marinaki (2008b) se propone una estrategia híbrida que combina tres métodos: Partícula Swarm, Greedy Randomized Adaptative Search Optimization (GRASP), y Expanding Neighborhood Search (ENS). La metodología se valida con los casos de Perl Y B y son comparables con los hallados en Barreto et al. (2007).

Una formulación bi-nivel para el LRP es propuesta en Marinakis y Marinaki (2008a) y se resuelve mediante una técnica genética híbrida que usa GRASP y ENS. Estos son descritos en Marinakis y Marinaki (2008b) para generar la población inicial. La recombinación se implementa con base en Taillard et al. (2001) usando Memoria Adaptativa. En esta dirección, la mutación se implementa cerrando y abriendo depósitos, y la metodología se aplica a un problema real de distribución de madera en Grecia. En comparación con el trabajo de Barreto et al. (2007), se mejoran los resultados de Perl y B, y no reportan los tiempos computacionales.

Un algoritmo de «recocido simulado» usando una longitud de cadena fija de identificadores de depósitos y clientes es propuesta por Yu et al. (2010). La solución de inicio se construye con una heurística golosa con base en criterios de proximidad. Luego, se aplica el LKH. Los operadores de búsqueda en vecindario aplicados son: inserción, intercambio y 2-OPT. Las capacidades de depósitos o vehículos que no se cumplen se reparan con vehículos o depósitos adicionales. La metodología se valida con las casos TB, B y PPW. Esta propuesta obtiene excelentes resultados respecto a la literatura, sin embargo, sus tiempos computacionales son mayores que los presentados en Tuzun y Burke (1999); Prins et al. $(2007,2006)$ y Bouhafs et al. $(2006)$

En Stenger et al. (2011) se presenta un híbrido entre GRASP y VNS. Inicialmente, el GRASP usa RECWA, una búsqueda local golosa con 2-OPT y relocalización e intercambio como estrategias inter-ruta. Las configuraciones infactibles son penalizadas mediante un mecanismo dinámico. Después de aplicar GRASP, se reserva en memoria las tres mejores configuraciones halladas para agregarlas en las etapas posteriores del algoritmo. Se hace un análisis del desempeño donde se concluye que es recomendable minimizar el número de depósitos a abrir. Los casos de prueba utilizados TB, B y PPW superan las respuestas obtenidas en Duhamel et al. (2010), Prins et al. (2006) y Yu et al. (2010) en calidad y tiempo computacional.

En Escobar et al. (2013) se propone un algoritmo «búsqueda tabú», potenciado con estrategias granulares para realizar búsquedas con criterios de sensibilidad inteligentes, utilizando estrategias de diversificación embebidas en un algoritmo de una búsqueda local iterada. La etapa de perturbación solo se aplica si después de un número de iteraciones no se mejora la respuesta. Se obtienen todos los óptimos de la literatura en tiempos computacionales muy cortos. 
Una nueva versión del algoritmo Particle Swarm Optimization (PSO) es propuesta en Marinakis (2015), se aplica al CLRP, y al problema de localización y enrutamiento con demandas estocásticas (CLRPSD). De este modo, se combinan tres estrategias diferentes, integradas en el PSO al implementar exploración de vecindarios locales y globales.

Un algoritmo de «búsqueda tabú» para resolver el CLRPSD que considera entregas y recogidas, y compuesto de tres etapas se propone en Huang (2015). Las etapas corresponden a la selección de ubicación, el agrupamiento de clientes y el enrutamiento de vehículos. Allí se valida la metodología con casos de prueba de la literatura especializada.

Un «algoritmo memético» es propuesto en Duhamel et al. (2010), el cual consta de las siguientes etapas: i) Codificación. En este se usa un solo vector para representar la solución global del problema. ii) Usar tres heurísticas clásicas y el RECWA para generar las configuraciones de la población inicial. iii) Utilizar torneo para la selección. iv) Recombinación. Aquí se emplea la denominada Partially Mapped Crossover (PMX). v) Se aplica un mecanismo de reparación mediante «búsqueda local». En este punto se valida con los casos de prueba TB y PPW. Los resultados son un poco mejores que los encontrados en Prins et al. (2006); sin embargo, no se alcanzan los presentados en Prins et al. (2007) y Duhamel et al. (2008). Sobre los casos tipo B, la metodología propuesta mejora la calidad de todo; no obstante, el tiempo computacional es mayor.

En Duhamel et al. (2010) se propone un GRASP llamado Evolutionary Local Search (ELS). Esta consiste en la implementación de soluciones de inicio, es decir, se usa el RECWA y dichas soluciones son mejoradas usando la «búsqueda local» propuesta en Prins et al. (2006). La solución encontrada es transformada en un tour gigante. Luego, se obtienen nuevas soluciones mediante un operador de mutación. Así, la cantidad de nuevas soluciones es un parámetro que debe calibrarse mediante el tamaño de la lista tabú. La selección del depósito se hace mediante una lista tabú. Posteriormente, se segmenta en tour usando los conceptos propuestos en Prins (2004). El criterio de parada corresponde al número definido para el GRASP. La metodología se prueba en las casos TB, B y PPW. Se mejoran algunas respuestas de las alcanzadas por Prins et al. (2006) y Prodhon y Prins (2008) con tiempos computacionales mayores.

En Mehrjerdi y Nadizadeh (2013) se propone una heurística de clusterización combinada con «colonia de hormigas». La metodología se valida comparándose con Prins et al. (2006), Barreto et al. (2007) y Marinakis y Marinaki (2008a). Esta alcanza sus respuestas sin entregar los tiempos computacionales usados.

Un algoritmo de Algoritmo de Búsqueda en Vecindario Variable combinado con Vecindario Variable Descendente se propone en Derbel et al. (2011). Luego, se aplica una etapa de perturbación repetitivamente, usando movimientos de inserción intra- e inter-ruta. Lo anterior permite infactibilidades con costos de penalización. Este autor compara los resultados con Prins et al. $(2006,2007)$ y Yu et al. (2010) en las casos B y no entrega tiempos de cómputo. 
En Jarboui et al. (2013) se proponen varios algoritmos VNS que usan Variable Neighborhood Descendent (VND) como componente de «búsqueda Local», considerando cinco tipos de vecindarios para el VND. En los primeros cuatro, aplica inserción, intercambio, invertir secuencias; usados como estrategias inter- e intra-ruta. En el quinto, se abren y cierran depósitos uno a uno de forma aleatoria. En la perturbación, se realizan movimientos de inserción en los vecindarios, cambios de depósitos entre los vecindarios y una combinación entre inserción de clientes e intercambios de depósitos de forma repetida. Las perturbaciones semi-aleatorias aplicadas son las que presentan los mejores resultados. Como casos de prueba, utiliza TB y PPW sin considerar la capacidad de los vehículos.

Por su parte, Quintero-Araujo et al. (2017) proponen, una metaheurística de dos fases para resolver el CLRP. En la primera fase, también conocida como «soluciones jerárquicas», los autores determinan los depósitos a ser abiertos y luego se asignan los clientes a los depósitos más cercanos para así generar un conjunto de soluciones iniciales del problema a través de una heurística de enrutamiento rápido. En la segunda fase, se refinan las soluciones encontradas en la primera fase a través de un procedimiento de perturbación a la asignación de un cliente, seguido de una heurística de enrutamiento intensiva. Los autores comprueban que la metaheurística propuesta es prometedora debido a que los resultados obtenidos son equiparables a los de la literatura, y algunos con diferencias del $0.4 \%$.

\subsubsection{Técnicas math-heurísticas para resolver el CLRP}

Un algoritmo que combina una heurística golosa como solución de inicio y luego aplica Lagrangian Relaxation (LR) y Granular Tabu Search (GTS) es propuesto en Prins et al. (2007). El procedimiento consta de varias fases, así: i) Cada ruta de la solución actual es un super-nodo y se presenta como un FLP que se resuelve con el LR. ii) La solución es mejorada resolviendo el MCVRP con el GTS donde se permiten infactibilidades penalizadas en la función objetivo. iii) La información de los arcos más visitados es almacenada para ser usada en las fases siguientes. Si la solución actual no mejora en las iteraciones finales, se crean nuevas rutas donde se les da prioridad a los arcos más visitados. Los resultados obtenidos mejoran las respuestas de Tuzun y Burke (1999), Barreto et al. (2007) y Prins et al. (2006).

Un algoritmo de dos etapas considerando flota heterogénea es propuesto en Chen y Ting (2007). El paso inicial es determinar qué depósitos abrir; posteriormente, se realiza la asignación de los clientes a los depósitos; finalmente, se resuelve el VRP correspondiente de cada depósito. En la Etapa nro. 1 el problema de SSCFLP es resuelto usando LR, previa obtención de un límite superior para SSCFLP, obtenido con una heurística golosa. En la Etapa nro 2, se encuentran soluciones para los VRP asignados a los depósitos abiertos, solo permitiendo soluciones factibles mediante la heurística del vecino más cercano y después de una etapa de mejoramiento aplicando el algoritmo de recocido simulado. La estrategia de intercambio de clientes entre rutas asignadas a depósitos diferentes presenta un 
buen efecto en la respuesta final. Se mejoran resultados y tiempos computaciones de varias casos, entre ellos, los siguientes: Perl y Daskin (1984), Hansen et al. (1994), Wu et al. (2002), Wang et al. (2005) y Barreto et al. (2007).

Una formulación lineal entera mixta se presenta en Özyurt y Aksen (2007). La técnica de solución propuesta descompone el problema en dos subproblemas: Localización de depósitos, -Facility Location Problem (FLP)-y el MDVRP. El primero es resuelto con un solver y el segundo usando el algoritmo de «búsqueda tabú» con estrategias de oscilación para permitir infactiblidades en cuanto a la capacidad de los depósitos. Este último incluye heurísticas de inserción y de vecino más cercano. Se mejoran las soluciones actuales conocidas para las instancias TB.

En Lopes et al. (2008) se propone una heurística secuencial (rutear primero, asignar después). Se implementan cuatro tipos de métricas de agrupamiento, tomadas de Barreto et al. (2007). Las rutas se construyen usando un modelo lineal entero de dos índices, resuelto con un software comercial, solo en casos de clústeres de 40 clientes o menos. Para tamaños mayores, se usan heurísticas de inserción que determinan el próximo cliente con base en criterios del algoritmo del ahorro y del vecino más lejano. El tour es mejorado usando 3-OPT. Para la fase de localización se resuelve el problema de localización-asignación usando un software comercial. Se valida la propuesta con las instancias tipo B.

Una heurística de tres etapas es propuesta en Lam et al. (2009) y consta de lo siguiente: i) Con base en la capacidad del vehículo se agrupan los clientes mediante una medida de distancia. ii) Se usa heurística de LKH (Lin y Kernighan, 1973) para encontrar la secuencia de ruta de cada grupo de clientes. El resultado anterior es un dato de entrada para resolver un FLP. iii) Se realiza una búsqueda local con movimientos de reubicación de clientes (denominados en inglés como string-relocation) e intercambio de clientes (del inglés string-exchange). Los resultados se validan con las instancias TB y B. Este método entrega resultados en menos de un minuto en casos de 150 clientes y 20 depósitos. La calidad de las respuestas no supera los resultados presentados en Prins et al. $(2007,2006)$ y Prodhon y Prins (2008).

En Contardo et al. (2014b) se presenta una combinación de GRASP con una heurística de generación de columnas. El procedimiento consta de cuatro partes: $i$ ) En la primera etapa se implementa el RECWA. ii) Luego, se ejecuta un ILS usando siete tipos de vecindarios locales de forma ordenada y cíclicamente. iii) Después, se resuelve PLEM para secuenciar los clientes. iv) Finalmente, se aplica una heurística para generar cortes y columnas. Esas columnas pueden ser tramos de ruta para insértalas en rutas existentes, las cuales son generadas con «búsqueda tabú». Se usan los cortes propuestos por Contardo et al. (2013). Las etapas i) y ii) son ejecutadas una vez al inicio del algoritmo, las etapas iii) y iv) se repiten hasta que se cumple el criterio de parada definido previamente.

En Alvim y Taillard (2013) el algoritmo está basado en el algoritmo Partial Optimization Metaheuristic Under Special Intensification Condictions (POPMUSIC), cuya filosofía es particionar el problema y mejorar cada 
sub-problema antes de entregar una solución global (Taillard, 1993; Taillard et al., 2001). Este tiene las siguientes etapas: i) Mediante Capacitated P-Median Problem (CPMP) se determina el número de centros. ii) Luego, todos los clientes son asignados a los centros lo cual da como resultado supernodos que fraccionan en subgrupos con base en la capacidad del vehículo. iii) Para cada subgrupo se resuelve nuevamente el CPMP usando el método del gradiente sin permitir infactibilidades. $i v$ ) Los TSP de cada subgrupo resultante son resueltos considerando los costos de apertura. El algoritmo abre un depósito para cada clúster y luego, con una estrategia golosa, va asignándolos a los depósitos. v) Solo se permiten soluciones factibles para los MDVRP identificados. Para validar la metodología se prueba con los casos propuestos por Duhamel et al. (2010) y Yu et al. (2010).

Una math-heurística de dos etapa es propuesta por Escobar et al. (2013). La etapa inicial corresponde a la construcción de un tour gigante hallado con la heurística de Lin Kernighan Helsgaun (LKH). Luego el tour es dividido con base en la capacidad del vehículo. Posteriormente, el SSCFLP es resuelto usando un software estándar para obtener la asignación óptima del clúster a los depósitos. En la segunda etapa, la solución se lleva a una etapa de mejoramiento usando los algoritmos de librería para VRP disponible (Groër et al., 2010). En el proceso de búsqueda son permitidas soluciones infactibles, penalizadas en la función objetivo. Al inicio, se reduce el número de rutas eliminando las rutas de menor demanda atendida. Esos clientes son insertados en otras rutas buscando minimizar el impacto en la función objetivo. Los resultados obtenidos se mejoran aplicando un algoritmo Granular Tabu Search (GTS) (Toth y Vigo, 2003), El -GTS - consta de inserción, intercambio, 2-OPT, reubicación e intercambio de secuencia con dos clientes de forma iterativa. El grafo disperso del GTS se define con base en la sumatoria promedio de los arcos obtenidos de la solución actual. Para diversificar la búsqueda, los autores alteran el grafo disperso, usando penalidades dinámicas para las alternativas infactibles; se revisan las mejores asignaciones de las rutas a los depósitos mediante los postulados de Groër et al. (2010), es decir, por un número determinado de iteraciones. Finalmente, cuando la respuesta no es mejorada se hace una perturbación usando CROSS-Exchange, similar al descrito en Escobar et al. (2013). Se usan como casos de prueba TB, B y PPW. Se encuentra mejor desempeño computacional en cuanto a tiempo que en Duhamel et al. (2010) y Yu et al. (2010) para muchas de las instancias consideradas.

Bernal-Moyano et al. (2017) presentan una comparación de tres algoritmos granulares de trayectoria para la solución del CLRP con flota heterogénea. Los algoritmos que se comparan son: «recocido simulado granular» (GSA), «búsqueda de vecindario variable granular» (GVNS) y «búsqueda tabú granular probabilística» (PGTS). Gracias al concepto de granularidad, empleado en los tres algoritmos, los movimientos menos favorables son descartados dentro de un subconjunto del espacio de solución. Los autores demuestran que el GSA es capaz de obtener soluciones de muy buena calidad en tiempos de cómputo reducidos, en comparación con los dos restantes.

Ospina-Toro et al. (2017) propone una metodología para identificar rutas alimentadoras en zonas no 
conectadas en relación con un sistema de transporte masivo con el fin de aumentar la cobertura del servicio y mejorar el nivel de ocupación del sistema. La metodología propuesta consta de dos etapas: i) Estructurar escenarios de áreas no conectadas al sistema de transporte y ii) combinar técnicas heurísticas y exactas para resolver el problema de rutas alimentadoras. La metodología considera, dentro de sus restricciones, la duración de la ruta y la capacidad del vehículo alimentador. Para su modelamiento, se establece una analogía entre los problemas del transporte de pasajeros y el problema de localización y ruteo -Location Routing Problem (LRP)-, que usualmente es aplicado a problemas de transporte de mercancías. La metodología de solución propuesta es una math-heurística que combina las heurísticas Lin-Kernighan-Helsgaun (LKH) y de ahorros con el algoritmo de ramificación y corte, Branch and Cut, aplicado sobre un modelo lineal entero mixto de partición de conjuntos (Set Partitioning) para LRP. Esta propuesta metodológica es validada con casos de prueba reales del sistema de transporte masivo de la ciudad de Pereira (Megabús), donde se consideran algunas zonas no conectadas del Área Metropolitana Centro-Occidente, localizada en el Eje Cafetero colombiano.

\subsubsection{Planteamientos multiobjetivo para el LRP}

El desarrollo económico de un país puede ser medido con diferentes indicadores, uno de ellos es la cantidad de transacciones del transporte de carga. Sin embargo, este a su vez, tiene asociado un gran efecto en el cambio climático. Por tanto, es un reto adicional a considerar en la logística de distribución, donde se deben agrupar nuevas tácticas multiobjetivo que no solo minimicen los costos operativos de transporte, sino también el impacto generado por los vehículos usados en la distribución de carga (Cuda et al., 2015).

En cuanto a las técnicas exactas, Toro et al. (2017b) presenta un modelo exacto basado en la condición de radialidad de la red de distribución. Allí se propone un nuevo modelo bi-objetivo del CLRP que considera el impacto ambiental. Se demuestra, a través de los diferentes resultados, que tanto la función de costos operativos, como la de consumo de combustible están en conflicto y para obtener el frente de Pareto se utiliza la técnica multiobjetivo Épsilon-Constraint, y se valida el modelo con los casos de Duhamel et al. (2010).

Otras soluciones que usan técnicas metaheurísticas se encuentran en Chen et al. (2018) donde proponen el problema de localización y enrutamiento de los vehículos con la carga completa minimizando la emisión de $\mathrm{CO}_{2}$. De esta índole, implementan un modelo bi-objetivo minimizando los costos y el impacto ambiental. La metodología de solución desarrollada es un híbrido multiobjetivo llamado NSGA-II-TS, el cual es una combinación del algoritmo NSGA-II con la heurística Tabu Search (TS).

En Lin y Kwok (2006) se resuelve el LRP considerando tres objetivos:

1) Minimización de costos fijos de apertura de depósitos y de los costos variables de las rutas. 
2) Balanceo de la carga de trabajo medida como el tiempo de cada ruta.

3) Balance de carga entregada en cada ruta.

Lo anterior, se resuelve mediante una técnica híbrida de tres etapas combinando «búsqueda tabú» y «recocido simulado»: i) Se determinan qué depósitos se deben abrir con base en las distancias promedio de los clientes. ii) Se enruta cada MDVRP considerando flota heterogénea, usando el algoritmo del ahorro y el algoritmo del vecino más cercano, mejorado con una estrategia de «búsqueda local» considerando movimientos inter- e intra-ruta. iii) Con el fin de permitir el uso de múltiples vehículos, se resuelve un problema de empaquetamiento en contenedores para cada depósito, donde el contenedor es el vehículo y la capacidad del vehículo corresponde al tiempo máximo de la ruta con base en el horario de trabajo del conductor. Se construye la frontera eficiente teniendo presente los tres objetivos. Los casos de prueba que se utilizan tienen en promedio 10 o 20 depósitos con un número de clientes de 100 a 200. La comparación de ambas metodologías entrega mejores resultados cuando se usa el «búsqueda tabú».

Cinco objetivos son considerados en Caballero et al. (2007). Allí se implementa la propuesta para resolver el LRP en la incineración de basuras en una región al sur de España. Los objetivos consideran minimizar: 1) los costos de apertura de los hornos incineradores, 2) los costos de las rutas, 3) el grado de rechazo de las ciudades afectadas por el tránsito de los vehículos recolectores, 4) el grado de rechazo por la apertura de los hornos en las regiones afectadas, 5) el grado máximo total asociado a la ciudad más afectada por el transporte de los residuos. La técnica de solución propuesta es una metaheurística usando memoria adaptativa. Esta considera mecanismos de exploración eficiente de vecindarios. Las soluciones de inicio se obtienen con un algoritmo goloso donde solo se consideran los costos de las rutas. Luego, se define el vecindario de búsqueda permitiendo un número fijo de iteraciones para la metaheurística teniendo presente los objetivos auxiliares y los diferentes elementos de «búsqueda local»; tres de ellos se encuentran asociados a las rutas y los otros tres a la prohibición o no de la ubicación de los depósitos. Las respuestas halladas se verifican para formar el conjunto de soluciones eficientes. Se describe el caso real pero no se comparten detalles de los casos de prueba. Los autores también validan la metodología con las casos de Albareda-Sambola et al. (2007) y encuentran soluciones de mejor calidad.

Un modelo bi-objetivo LRP en escenario estocástico es propuesto en Hassan-Pour et al. (2009). En su trabajo, los objetivos considerados son la minimización de los costos de apertura de depósitos y arcos activos, y la minimización de la probabilidad de que un cliente no sea atendido. El problema es resuelto en dos etapas: Inicalmente el SSCFLP se plantea como un PLEM, se resuelve usando un solver estándar; considerando restricciones de oportunidad,chance-constraint, asociadas a la probabilidad de que un cliente sea servido y debe ser mayor o igual a un valor fijado. En la segunda etapa, se resuelve un MDVRP aplicando un recocido simulado mejorado en su búsqueda local con intercambio de clientes entre rutas y la heurística 2-OPT. La metodología se valida con casos propios de los autores. 
Un modelo bi-objetivo para LRP con clientes opcionales es presentado en Tavakkoli-Moghaddam et al. (2010). Las funciones objetivo a optimizar son: Minimizar los costos de apertura de depósitos, los costos variables de rendimientos de los depósitos y los costos de las rutas; la segunda función objetivo es maximizar la sumatoria de demanda atendida de los clientes. Se propone un PLE y se plantean dos metaheurísticas: Muti-objective Scatter Search (MOSS) y un Tabu Search Elite (ETS). La filosofía del MOSS consiste en definir un límite inferior del número de depósitos, basado en la demanda total de los clientes y la capacidad de los depósitos. De acuerdo con ese valor, todos los depósitos se jerarquizan posteriormente, se resuelve el VRP correspondiente considerando la primera función objetivo. Luego, se asignan los clientes al depósito más cercano e implementa el algoritmo del ahorro. La solución es mejorada usando el 2-OPT; luego, se permite la apertura de un depósito más con el fin de evaluar el efecto de esa decisión sobre la función objetivo y se aplica nuevamente el algoritmo para asignar los clientes y definir las rutas. El ETS desarrolla las mismas estrategias del MOSS, pero refina las búsquedas con estrategias adicionales de intercambio, y define un criterio de aspiración con base en las distancias de la red. La metodología se valida con casos propios generados por los autores. Los mejores resultados se obtienen con la metodología MOSS. Para observar las tendencias y oportunidades de investigación en esta temática resulta interesante chequear los artículos de Prodhon y Prins (2014), Escobar et al. (2015) y Drexl y Schneider (2015)

\subsubsection{Aplicaciones del CLRP}

De las aplicaciones del problema se encuentran algunos reportes en la literatura que hacen referencia al mismo y entre los que se encuentran algunos documentos reservados para las fuerzas armadas (Chan y Baker, 2005), distribución de correspondencia en ciudades (Lin y Kwok, 2006), planeación y operación en labores de logística militar (Burks, 2006), instalación de hornos de incineración de basuras peligrosas y el enrutamiento de recolección de las mismas (Lopes et al., 2008), cadena de suministro de industria maderera (Marinakis y Marinaki, 2008a), distribución mercancía para cadenas de supermercados (Ambrosino et al., 2009), distribución de repuestos de vehículos (Schittekat y Sörensen, 2009), distribución de correos (Çetiner et al., 2010), planeamiento de misiones en exploraciones especiales (Ahn et al., 2012), ubicación de estaciones de intercambio de baterías para vehículos eléctricos (Yang y Sun, 2015).

En Yang y Sun (2015) se presenta un problema de localización y enrutamiento de vehículos eléctricos con estaciones de intercambio de baterías (BSS). El objetivo principal de la investigación propuesta por los autores es determinar una estrategia óptima de ubicación de los BSS y el conjunto de rutas óptimas formadas por una flota de vehículos eléctricos (EV). Para solucionar el problema planteado, los autores proponen dos heurísticas: la primera es una heurística de cuatro fases denominada SIGNALS y la segunda es una heurística de dos fases denominada 
«ahorro modificado por búsqueda tabú». En esta propuesta, se hace un análisis económico y ambiental que incluyen una comparación entre escenarios básicos y extendidos. Por su parte, Karaoglan y Altiparmak (2015) proponen un algoritmo memético para resolver el problema de localización y enrutamiento con backhauls mixtos, en el cual se pretenden encontrar las ubicaciones de los depósitos y diseñar un conjunto de rutas ya que los puntos de recolección y entrega de cada cliente se deben realizar con el mismo vehículo. Para validar la eficiencia del algoritmo, los autores evalúan un banco de instancias clásicas del CLRP y se comparan con los resultados obtenidos a partir de un algoritmo exacto Branch and Cut.

Por otro lado, en Bashiri et al. (2016) se presenta un CLRP con asignación de vehículos auxiliares en donde la longitud de cada ruta no se restringe por la capacidad del vehículo. En el estudio realizado por los autores se consideran dos tipos de vehículos: $i$ ) vehículos con mayor capacidad y costo fijo asociado y ii) vehículos auxiliares con menor capacidad y su respectivo costo fijo. La característica operativa radica en que cada vehículo auxiliar puede transferir mercancía desde los depósitos hasta vehículos de carga mayor; por lo cual, no pueden atender a los usuarios, y además, funciona como estrategia alternativa para cubrir limitantes de capacidad. Para comprobar la eficiencia del modelo propuesto, los autores usan un banco de instancias clásicas adaptadas al problema que se resuelven a través de una técnica exacta. Un estudio muy parecido al anterior fue el realizado por Yakıcı (2016) donde se resuelve un CLRP para drones. A diferencia del CLRP tradicional, el autor formula el problema como un PLE en el que maximiza la puntuación total recopilada de los puntos óptimos visitados por los drones. Las rutas de vuelo de cada dron se originan a partir de asignación de bases, y posteriormente, se asigna el orden de visita de los puntos de interés a cada base. Para la solución del problema, se propone un algoritmo poblacional «colonia de hormigas». Luego, se corrobora que la metodología y el algoritmo propuesto presentan un buen desempeño en instancias de alta complejidad computacional.

Algunas aplicaciones del CLRP, como la realizada por Pourreza et al. (2017), demuestran que el cliente es el elemento más relevante en la cadena de suministros. Por ejemplo, en el texto de Pourreza et al. (2017), se plantea un CLRP que considera la satisfacción de clientes bajo riesgo de interrupción en centros de servicios médicos (MSC). El objetivo principal del problema es encontrar las mejores ubicaciones de los MSC y las mejores rutas de vehículos para atender a los pacientes. Para ello, los autores proponen un algoritmo híbrido NSGA-II el cual es comparado con el NSGA-II tradicional y, para ajustar los parámetros de ambos algoritmos, se utilizan el método Taguchi. Por otro lado, en Zhao et al. (2018) se presenta un two-echelon CLRP con flota heterogénea para el diseño óptimo de una red de logística urbana, con el propósito de generar alianzas de entrega conjunta en la industria de paquetería en China. El objetivo general del problema planteado por los autores es establecer el conjunto de depósitos intermedios o satélites que deben ser abiertos, y la asignación ordenada de puntos de logística de la ciudad para minimizar los costos de apertura de los satélites y el costo de viaje de las rutas. Para solucionar el problema planteado, los autores desarrollan 
un algoritmo heurístico el cual es comparado con tres enfoques previamente publicados para el tratamiento del mismo problema. Lo anterior los lleva a comprobar, de manera exitosa, la eficiencia y efectividad del algoritmo en cuanto a calidad de solución y los tiempos de cómputo. Una investigación semejante a la de Zhao et al. (2018) se propone en Mohamed et al. (2019), donde se resuelve un two-echelon CLRP estocástico con múltiples periodos.

En el primer nivel, se decide el número y las ubicaciones de los centros de distribución y de los satélites en cada periodo. La distribución de centros de distribución y satélites se hace de forma óptima, teniendo en cuenta la demanda estocástica de cada cliente la cual es variable en el tiempo. En el segundo nivel, se diseña un conjunto de rutas que van desde los satélites hasta los clientes. El problema propuesto por los autores es modelado como un problema lineal estocástico de dos etapas con recurso entero, donde en la etapa uno se toma la decisión de ubicaciones y capacidad por periodo; y en la segunda, denominada recurso, se diseña el conjunto de rutas. Para la solución del problema estocástico, los autores implementan en la primera etapa un algoritmo de descomposición de Benders y para el subproblema de enrutamiento resultante (un CVRP), se aplica un algoritmo exacto Branch and Cut and Price.

Otra investigación del CLRP en la que también se consideran demandas estocásticas es realizada por Quintero-Araujo et al. (2019). El objetivo principal, además de determinar los depósitos a abrirse y las rutas a ejecutar, consiste en realizar acciones correctivas en caso de que se presenten fallos por exceso de carga. Para la solución del CLRP con demandas estocásticas, se usa el ILS sobre el cual se usa una política de recurso denominada «stock de seguridad para minimizar los fallos por ruta». En Pekel y Kara (2019), en lugar de resolverse un problema estocástico, se resuelve un CLRP difuso que se soluciona mediante una heurística híbrida que combina un algoritmo de Búsqueda de Vecindario Variable (VNS) y uno de Búsqueda Local Evolutiva (ELS); se considera que los tiempos de viaje entre clientes y depósitos, y las demandas de los clientes son variables difusas. El objetivo es minimizar el costo de viaje, el costo adicional por apertura de centros de distribución, los costos de espera del vehículo y los costos de demora. Al igual que Pekel y Kara (2019), Nadizadeh y Kafash (2019), se resuelve un CLRP difuso pero con demandas simultáneas de recolección y entrega que, desde el punto de vista operativo, se denomina como problema de logística inversa. En la investigación, se considera que las demandas de entrega y recogida son variables difusas. Para el modelamiento del problema, los autores diseñan un modelo que incluye un conjunto de restricciones difusas, basadas en la teoría de la credibilidad. Para la solución del CLRP propuesto, los autores implementan un algoritmo de clusterización goloso que incluye cuatro fases iterativas. Para validar el funcionamiento del algoritmo y la metodología, se consideran instancias clásicas del CLRP.

Los casos de prueba usados para validar metodologías que permiten resolver el CLRP, de mayor uso en la literatura, se listan a continuación:

Perl: planteadas en la tesis doctoral de Perl y Daskin (1984). 
TB: propuestas por Tuzun y Burke (1999).

B: propuestas y usadas en Barreto et al. (2007).

PPW: planteadas por Prins et al. (2006).

ABR: popuestas por Akca et al. (2009).

BMW: presentadas por Baldacci et al. (2004).

Todas disponibles en las direcciones web del pie de página. ${ }^{1}$

\subsubsection{Planteamientos del problema Two-Echelon Vehicle Routing Problem (2E-VRP)}

Por otro lado, en la industria de transporte se pueden encontrar dos estrategias de distribución de mercancía: envío directo, en donde la carga va desde los depósitos hacia los clientes (enfoque tradicional); y distribución multinivel, o multi echelon, en el cual la carga que se le entrega a los clientes debe empezar en un origen y pasar a través de un conjunto de satélites o depósitos intermedios (Perboli et al., 2011). Una de las ventajas de la distribución multinivel o multi echelon es que permite tener en cuenta factores como control en el tráfico de carga e impacto ambiental (Crainic et al., 2004). El primer registro conocido en la literatura sobre el problema de enrutamiento multi echelon es el de Jacobsen y Madsen (1980). Los autores resuelven un problema de distribución de periódicos con puntos estratégicos de transferencia de material sobre el cual se deben tomar tres decisiones: i) cantidad y ubicación de los puntos de transferencia, ii) rutas óptimas que deben atender los puntos de transferencia, teniendo en cuenta que dicho conjunto de rutas empiezan su recorrido desde la imprenta y iii) rutas óptimas que deben servir a los clientes finales, teniendo en cuenta que estas rutas salen desde los puntos de transferencia. El problema es resuelto a través de tres heurísticas propuestas por los autores. Por ende, el problema de enrutamiento de vehículos con dos niveles, o Two-Echelon Vehicle Routing (2E-VRP), consiste en el diseño de $m+n$ rutas que entregan una cantidad de carga a un depósito intermedio y desde ese depósito a los clientes ( $m$ es el número de rutas en el nivel 1 y $n$ es el número de rutas en el nivel 2).

A pesar de que la función objetivo del 2E-VRP solo minimiza costos de transporte del primer y segundo nivel, tiene la deficiencia de no tener en cuenta parámetros de instancias en el costo global, es decir, parámetros como distribución de los clientes, reglas de ubicación de los depósitos intermedios o satélites, ubicación del depósito principal, cantidad de satélites, razón de accesibilidad a los satélites, y costo medio de viajes entre satélites y clientes. Por ese motivo, Crainic et al. (2010) presenta un 2E-VRP en el cual se realiza un análisis de satélites teniendo en cuenta los parámetros omitidos en Jacobsen y Madsen (1980).

Al ser una variante del problema tradicional de enrutamiento capacitado VRP, se clasifica entonces como

\footnotetext{
${ }^{1}$ http://claudio.contardo.org/instances/; http://sweet.ua.pt/sbarreto/private/SergioBarretoHomePage.htm/
} 
un problema de complejidad computacional NP-duro; por lo cual, en sus soluciones, se suelen encontrar técnicas aproximadas que permiten encontrar buenos resultados en tiempos de cómputo razonables. Por ejemplo, Perboli et al. (2011) presenta una familia de problemas de enrutamiento de dos niveles. El propósito de la investigación realizada por los autores es establecer un modelo matemático del 2E-VRP y resolverlo por medio de una técnica exacta y aproximada. Para la técnica exacta, se formulan desigualdades válidas que ayudan a la relajación del problema; para la aproximada, se propone una math-heurística que acoge parte del modelo exacto. Para validar los resultados, se evalúa un conjunto de instancias con hasta 50 clientes y 4 satélites sobre las cuales se obtienen resultados de muy buena calidad. En Crainic et al. (2011), se presenta una familia de heurísticas con múltiples incios que se basan en transferencia separada deposito-satélite y entrega separada satélite-cliente. Bajo este esquema, se obtienen dos sub-problemas de enrutamiento que pueden ser resueltos de forma iterativa. La característica principal de los algoritmos que se proponen radica en las diferentes estrategias de diversificación y reglas de búsqueda de viabilidad que mejoran de forma significativa los métodos propuestos por Perboli et al. (2011). Crainic et al. (2013) proponen una metaheurística denominada procedimiento goloso de búsqueda aleatoria adaptativa o GRASP. El algoritmo se combina con el método de vinculación de ruta para resolver de forma efectiva el 2E-VRP. Para ello, se implementa un GRASP y varios procedimientos de búsqueda local en secuencia. Luego, mediante un proceso de vinculación de ruta, la solución resultante se vincula a una solución elite; además, se implementa una búsqueda de factibilidades dentro del proceso de vinculación por ruta para evitar soluciones infactibles del problema. Para validar la metodología, se utiliza un banco de instancias con hasta 50 clientes y 5 satélites. Asimismo, esta demuestra ser muy superior a la metodología planteada por Crainic et al. (2011) y equiparable a la de Jepsen et al. (2013), en la cual se usa un algoritmo exacto Branch and Cut con esquemas de ramificación muy promisorios. En Rahmani et al. (2016), se plantea un problema de localización y enrutamiento de dos niveles (2E-LRP) en el cual se debe determinar la cantidad de satélites a ser usados y el número de depósitos que van a servir a los satélites. Dentro del problema, se incluyen tres restricciones adicionales: múltiples productos, entrega y recogida de mercancía, y uso de rutas en el segundo nivel. El 2E-LRP con múltiples entregas y recogidas se modela como un Problema Lineal Entero Mixto (PLEM) para instancias pequeñas, resuelto con CPLEX. Para instancias más grandes, se usa un enfoque heurístico basado en las variantes del vecino más cercano por medio de técnicas de agrupación. Breunig et al. (2016) proponen dos problemas de optimización combinatorial que surgen dentro del contexto de logística urbana o de ciudades. El primero es un 2E-VRP y el segundo es un 2E-LRP. Ambos problemas se resuelven con una meta-heurística híbrida que combina búsquedas locales enumerativas con principios de destrucción y reparación, y además se proponen unos operadores adicionales con el fin de optimizar la selección de satélites. Los autores logran comprobar la eficacia de la metodología al obtener resultados de muy buena calidad en tiempos de cómputo relativamente cortos. Una investigación similar a la de Breunig et al. (2016) es 
realizada por Grangier et al. (2016), en donde se aborda un 2E-VRP pero con múltiples viajes y sincronización satelital. Para resolver el problema, los autores usan un algoritmo de búsqueda adaptativa de vecindario grande en conjunto con heurísticas de reparación y destrucción personalizadas, y búsquedas de factibilidad. En Wang et al. (2017) se aborda un 2E-VRP con demandas estocásticas. Al ser un problema de programación lineal entera, se deben lograr dos objetivos a saber: minimizar el costo global de viaje y los costos asociados a posibles fallos por ruta. Para resolver el problema, los autores proponen un algoritmo genético con un esquema simple de codificación y decodificación, un operador de cruzamiento modificado y un operador de mutación basado en la selección de satélite. Se comprueba que el algoritmo propuesto puede encontrar soluciones de muy buena calidad para el problema planteado. En Belgin et al. (2018), se resuelve un 2E-VRP con entrega y recogida de mercancía simultánea. El problema se resuelve desde dos ópticas: i) un problema PLEM basado en notación de nodos, el cual se refuerza con tres desigualdades validas clásicas de la literatura y ii) un algoritmo heurístico de descenso de vecindario variable (VNS) con búsqueda local (LS). Se demuestra la efectividad de las dos ópticas para resolver el problema y se comprueba la versatilidad de ambas técnicas para adaptarse a problemas de la vida real.

En cuanto a técnicas de solución exacta, se encuentran las siguientes aproximaciones: en Perboli et al.(2018b) se adaptan un conjunto nuevo de desigualdades, válidas para el 2E-VRP. Estas se obtienen a partir de unas variantes existentes para el problema de enrutamiento clásico. El problema se resuelve con un algoritmo Branch and Cut. El demuestra ser eficiente para instancias de tamaño medio, reduciendo significativamente la brecha en cuanto a la calidad de solución para instancias grandes. Dellaert et al. (2019) se propone un algoritmo exacto Branch and Cut para resolver el 2E-VRP. Para ello, la formulación matemática del problema es dividida en dos: $i$ ) recorridos de primer y segundo nivel y ii) descomposición de rutas en primer y segundo nivel. Se demuestra la gran eficiencia del algoritmo al resolver instancias con hasta 100 clientes y 5 depósitos, siendo la primer técnica exacta en lograr resultados tan efectivos y comparables a los de Crainic et al. (2013).

\subsubsection{Aplicaciones del 2E-VRP}

Muchas de las aplicaciones del 2E-VRP están relacionadas con la mejora de indicadores en logística de última milla. En esa dirección, se pueden encontrar algunas investigaciones como la realizada por Soysal et al. (2015) en donde se resuelve un 2E-VRP que depende del tiempo y que considera efectos contaminantes. En esta investigación se considera el tipo de vehículo, la distancia recorrida, la velocidad de los vehículos, la carga y las zonas horarias múltiples. En Li et al. (2016a) se presenta un problema de enrutamiento táctico a corto plazo denominado «enrutamiento de vehículos con 2 niveles y restricciones de tiempo en sistemas de entrega de línea» o Two-Echelon and Time-constrained Vehicle Routing Problem (2E-TVRP). La solución del problema se da a través de una heurística de ahorro mejorada por una 
fase de búsqueda local. Los resultados obtenidos por los autores demuestran la eficiencia en reducción de emisiones contaminantes para sistemas de distribución. En Wang et al. (2018) se presenta un 2E-VRP que considera el impacto ambiental. El objetivo del problema planteado es minimizar el costo de combustible, el costo de manejo y la suma del salario de todos los trabajadores. Para solucionar el 2E-VRP con impacto ambiental se usa el VNS en conjunto con una técnica exacta cuyo modelo matemático se basa en la formulación de arcos. La técnica exacta tiene dos objetivos dentro de la metodología planteada: i) encontrar soluciones de buena calidad cuando el VNS encuentra óptimos locales y ii) encontrar las rutas óptimas del primer nivel. Se comprueba la efectividad de la math-heurística usada al mejorar la solución de 13 instancias clásicas del 2E-VRP de las 234 conocidas. Otra aplicación relevante es la que se presenta en Luo et al. (2017) en la cual se investiga un problema de enrutamiento de dos niveles cooperado, es decir, un vehículo terrestre (GV) trabaja de forma cooperada con un dron (UAV) con el fin de que este último termine la entrega de mercancía en los puntos que el GV no puede cubrir. Para ello, se formula un problema lineal entero binario que se resuelve a través de dos heurísticas. Por su parte, Eitzen et al. (2017) plantea 2E-VRP multiobjetivo de múltiples proveedores. En la investigación, se presentan cuatro objetivos que deben ser minimizados: i) el costo del primer nivel, ii) el costo del segundo nivel, iii) el número de rutas en el nivel uno y dos, y iv) las emisiones contaminantes. Se comprueba que la cuarta función objetivo está en conflicto con los objetivos i) y ii), por lo cual, la solución es un frente de Pareto. En Zhou et al. (2018) se plantea un nuevo 2E-VRP que surge en la distribución de comercio electrónico de última milla. La característica principal del problema planteado es que los clientes pueden solicitar diferentes opciones de entrega, lo cual les puede permitir recoger sus paquetes en los satélites. Para resolver el problema, los autores proponen un algoritmo genético híbrido de múltiples poblaciones y una heurística eficaz que permite obtener soluciones iniciales de buena calidad. En Breunig et al. (2019), se presenta un nuevo problema del 2E-VRP. El problema planteado considera una flota de vehículos eléctricos que son indispensables para la operación de segundo nivel en las ciudades, con el fin de minimizar efectos contaminantes. Para solucionar el problema, se presenta una técnica math-heurística compuesta por un algoritmo de búsqueda de vecindario de gran tamaño (LNS) y un algoritmo exacto descompuesto que se encarga de enumerar las soluciones promisorias del primer nivel a través de unos límites funcionales y se encarga de ordenar las rutas de segundo nivel. Una de las características del problema es que permite evaluar el impacto sobre las fuentes óptimas de baterías en las redes de distribución de energía eléctrica.

\subsection{Problemas de enrutamiento con flota propia y subcontratada}

La logística de transporte, asociada a la entrega de paquetes, transporte de personas aéreo o terrestre y a la prestación de servicios tiene implícito el hecho de ser una actividad cambiante, donde el desempeño se ve afectado por variables como la demanda que puede llegar a ser difícil de predecir o establecer. También es necesario considerar que las flotas 
de vehículos podrían no siempre permanecer constantes ya que las mismas requieren de mantenimientos y de un recurso humano. Por estas razones, surge la necesidad de establecer un planteamiento del problema de enrutamiento nuevo, asociado al uso de flotas de vehículos subcontratadas las cuales permiten una mayor flexibilidad. Con base en esto han planteado las variantes como VRPPC y MDVRPPC. Estos problemas que consideran la flota propia y subcontratada no son comunes en la literatura.

\subsubsection{Problema de enrutamiento de vehículos con flota propia y subcontratada VRPPC}

Klincewicz et al. (1990) presentan un problema de decisión estratégico en el que se debe determinar la composición de la flota de forma periódica, que puede ser el uso de toda la flota privada, propia o subcontratada. También consideran la posibilidad del uso de una combinación de ambas. El modelo del problema considera un único depósito y plantea la solución dividiendo la demanda en zonas geográficas específicas que deberán ser atendidas por alguno de los vehículos (propio o subcontratado). En la función de costos, son considerados los valores fijos y variables por milla, asociados a cada tipo de vehículo utilizado.

En Chu (2005) se denomina el problema como truckload and a less-than truck load carrier. Es propuesto un modelo matemático lineal entero binario el cual soluciona casos hasta de 25 clientes con flota heterogénea. Asimismo, este estudio plantea una heurística de solución inicial que modifica el algoritmo de ahorros propuesto por Clarke y Wright (1964), adicionando una fase de mejoramiento.

En Ball et al. (1983), se realiza una de las primeras aproximaciones, motivada por un problema de planeación de entregas o envíos de una firma comercial, donde los vehículos deben hacer extensos recorridos para llegar a los clientes. Por lo anterior, se debió considerar en la solución incluir el pago y utilización de vehículos subcontratados. La investigación fue desarrollada gracias a la necesidad de una empresa comercial, la cual debía optimizar la forma de hacer entregas a una gran cantidad de clientes. La solución del problema consistía en obtener el tamaño óptimo de la flota sin exceder las restricciones de tiempo y asignar las rutas a los vehículos. Se describen algunas soluciones aproximadas y se plantean aspectos de la implementación.

En Bolduc et al. (2007) proponen una heurística mejorada denominada selection, routing and improvement (SRI). Después de obtenidas las soluciones iniciales, se realiza un mejoramiento para obtener la solución final. Este consiste en aplicar un intercambio de clientes por medio de un procedimiento denominado lambda-interchange. En Bolduc et al. (2008) se desarrolla una metaheurística que implementa perturbaciones para mejorar la exploración, esta metaheurística es denominada randomized construction improvement perturbation (RIP) y consiste en la construcción de una solución inicial con un algoritmo de ahorros en forma aleatoria que posteriormente tendrá dos etapas de mejoramiento. Esta metaheurística realiza una búsqueda local descendente basada en diferentes estructuras de 
vecindad con dos estrategias de diversificación: Un procedimiento aleatorio constructivo y un procedimiento de perturbación para escapar de resultados locales. La metodología se valida con instancias de flota homogénea y heterogénea.

Côté y Potvin (2009) desarrollaron una heurística basada en «búsqueda tabú» y encadenamiento de trayectorias o cadenas de expulsión. La integración de estos mecanismos permite encontrar mejores respuestas, particularmente, para los casos heterogéneos, propuestos por Bolduc et al. (2008), ya que se basa en el intercambio inter-ruta de clientes donde el número de estos que ingresa a la ruta es el mismo que sale y se ingresa a otra ruta. El vecindario del encadenamiento de trayectorias tiene la propiedad de modificar la asignación de clientes entre la flota propia y la flota subcontratada.

Potvin y Naud (2011) plantean una metaheurística basada en la metodología de «búsqueda tabú» con cadenas de eyección en la que se ordenan los clientes de acuerdo con una ponderación para asignarlos a la flota subcontratada. En relación con los clientes restantes, se crean rutas para ser atendidos por la flota propia a través de una heurística basada en los costos de viaje. Estas rutas serán la solución inicial que tendrá una fase de doble mejoramiento a través de la implementación de una búsqueda tabú en un vecindario estructurado. El modelo presentado permite la infactibilidad en la capacidad de los vehículos en la función objetivo a través de un parámetro de penalización. Para la verificación del desempeño, fueron usados casos homogéneos y heterogéneos y se obtuvo un mejoramiento en las soluciones. Sin embargo, los tiempos computacionales son significativamente más altos que los obtenidos en Bolduc et al. (2008).

Liu y Jiang (2012) consideran el VRPPC como close-open vehicle routing problem (COMVRP) para la solución del problema con rutas abiertas y cerradas. La función de costos es planteada con valores fijos y variables de acuerdo con los recorridos de los vehículos. Se propone un modelo matemático lineal entero mixto y un algoritmo memético para su solución. Para la verificación de rendimiento, son usadas las instancias propuestas en Augerat et al. (1998), adaptadas a las condiciones del problema. El modelo matemático fue implementado en CPLEX 12.2 con un límite de tiempo de 48 horas para las instancias entre 32 y 50 clientes. Lo anterior reportando unos GAP de $20 \%$ en promedio. El algoritmo memético es comparado con los valores encontrados y con el modelo exacto implementado en CPLEX para casos desde 32 clientes hasta 361 clientes y se reportan GAP de 0.3 a $13.88 \%$.

Euchi et al. (2013) proponen una metodología que usa un algoritmo de estimación evolutiva de densidad iterativa IDEA hibrido (IDEA/2-opt). Esta metaheurística es un tipo de algoritmo evolucionario en el cual una población inicial de individuos es mejorada por la aplicación de operadores estocásticos. Este algoritmo se ejecuta por etapas como: (1) selección de buenos candidatos (soluciones) desde una población de soluciones inicial generada aleatoriamente, (2) implementación de operadores de selección que permiten seleccionar las mejores soluciones 
de la población. Aquí es usado el procedimiento llamado «selección por presión hacia diversidad» de Bosman y Thierens (2002), (3) aplicación de modelo probabilístico que captura la importancia de la correlación de la distribución de la búsqueda, asignando altos valores de probabilidad a la solución seleccionada. IDEA construye un modelo probabilístico con los mejores individuos y luego toma muestras del mismo modelo para generar otros nuevos individuos y (4) generación de nuevos candidatos o descendencia desde la distribución estimada.

En su trabajo, Kratica et al. (2012) definen el problema como routing and carrier selection problem. El objetivo del problema es minimizar la función de costos que consta de tres partes: costos fijos debido al uso de los vehículos de la flota propia, costos variables de cada vehículo y costo de los recorridos realizados por la flota subcontratada. Presentan una heurística basada en un marco de trabajo de la búsqueda genética, donde se ordenan los clientes que no fueron atendidos por la flota propia en orden ascendente de acuerdo con sus distancias en relación con el depósito y, a través de ellas, dirige la búsqueda en el espacio de soluciones, aplicando los clásicos operadores genéticos de selección, recombinación y mutación. La metodología es validada con instancias homogéneas y heterogéneas, tomadas de Bolduc et al. (2008).

Goeke et al. (2019) propone un algoritmo exacto, branch-price-and-cut, donde incorporan técnicas recientes y utilizan ramificación adaptada con el fin de asegurar la integridad para resolver instancias pequeñas y medianas mientras que, en relación con las instancias grandes, proporciona limites inferiores estrechos logrando con esto una disminución significativa entre los espacios de las soluciones más conocidas. Adicionalmente, desarrollan una gran búsqueda de vecindario (LNS) como heurística, la cual propone un nuevo método de descomposición e implementan varios operadores de extracción e inserción teniendo en cuenta la decisión de subcontratación del VRPPC.

Sawadsitang et al. (2017b) proponen un VRPPC con ventanas de tiempo y demanda aleatoria llamado Optimal Delivery Planning (ODP) donde se minimiza el costo total mientras se cumplen las limitaciones de ventana del tiempo propuestas por el cliente. De este modo, evaluaron el desempeño usando la hoja de ruta real de Singapur. Sawadsitang et al. (2017a), por su parte, proponen la Planificación Óptima de Entrega Estocástica con Plazo (ODPD), el objetivo es permitir que el proveedor pueda planificar y optimizar la entrega de paquetes dentro de un plazo determinado, teniendo en cuenta la demanda y el tiempo estocásticos.

Euchi (2017) emplea, para la solución del VRPPC, un algoritmo de «búsqueda tabú» junto a vecindarios con cadenas de eyección. Esta metodología crea una sofisticada estructura de vecindad que permite una búsqueda eficiente en el espacio de soluciones, ya que incorpora la eficiencia del «búsqueda tabú» en la intensificación y la construcción de movimientos multifactoriales de los vecindarios con cadenas de eyección. Estas permiten varios grados de infactibilidad y generan potentes movimientos de intercambio.

Castaneda et al. (2020) desarrolla un algoritmo metaheurístico de optimización Iterated Local Search (ILS) el 
cual implementa el VNS. En la primera etapa del algoritmo, es considerada la obtención de una solución inicial con base en la heurística de ahorros modificada, la cual resulta ser determinante en el desempeño global de este. En la segunda etapa, se aplica la metaheurística ILS, la cual se fundamenta en conceptos de intensificación y diversificación, los cuales son aplicados a través de la heurística VNS y un esquema apropiado de perturbación, respectivamente. Una comprobación final al desempeño del algoritmo desarrollado se implementa usando instancias de referencia de la literatura. Los resultados de la metodología propuesta, basada en la metaheurística ILS, son comprobados con los obtenidos con métodos exactos, con el fin de validar la efectividad del método. Posteriormente, son usadas instancias de gran complejidad matemática donde los resultados obtenidos no presentan respuesta en la literatura especializada y se definen como nuevos Best Known Solution (BKS) para el problema estudiado.

Dabia et al. (2019) plantea una nueva variante para el VRPPC: un modelo de costos asociado a la carga asignada a los vehículos de la flota subcontratada. Este tiene en cuenta la variación del costo debido a la cantidad de carga y lo integra al diseño estratégico del enrutamiento de mediano plazo. Adicionalmente, tiene en cuenta ventanas de tiempo. A este problema lo denominan Rich Vehicle Routing Problem with Private Fleet and Common Carrier (RVRPPC).

\subsubsection{Problema multi-depósito de enrutamiento con flota propia y flota subcontratada (MDVRPPC)}

En Chu et al. (2019) se define una variante multidepósito del VRPPC, donde se permite realizar entregas y recogidas simultáneamente a los clientes. Sin embargo, no es presentado, de forma específica, cuáles clientes son atendidos por la flota subcontratada. Son propuestos un modelo matemático y una heurística constructiva simple. La validación es realizada con instancias hasta de diez clientes y dos centros de distribución.

Stenger et al. (2013) presentan un problema de enrutamiento asociado en las entregas finales o de última milla, donde los trayectos son inferiores a una milla y las cargas son pequeños paquetes. Este es denominado como problema de enrutamiento multicentro de distribución con flota propia y subcontratada - Multi-Depot Vehicle Routing Problem with Private Fleet and Common Carrier (MDVRPPC)-, presentado como una extensión del problema de enrutamiento con múltiples centros de distribución, donde los clientes pueden ser atendidos por la flota propia, situada en los centros de distribución de la empresa, o por vehículos subcontratados. En este trabajo se desarrolla un algoritmo basado en vecindarios de intercambio cíclico que incorporan un mecanismo adaptativo, considerando una etapa aleatoria de perturbación. Los estudios computacionales son realizados sobre dos conjuntos de casos desarrolladas por los autores. El primer conjunto de casos está conformado por 55 casos entre 5 y 20 clientes con tres centros de distribución propios y cuatro centros de distribución subcontratados. El segundo conjunto de casos es una adaptación de 33 casos tomados de Cordeau et al. (1997). 


\subsubsection{Problema de localización y enrutamiento con flota propia y subcontratada (CLRPPC)}

El CLRPPC es un área de interés que se considera como una nueva línea de investigación (Prodhon y Prins, 2014). Este caso se puede observar cuando el nivel de actividades aumenta como en el caso de la industria de servicios, donde aparecen de forma reiterada actividades como el mantenimiento y reparación de la flota de vehículos cuando la flota no es suficiente para atender la demanda de los clientes. Esta situación también se presenta en la logística de desastres, donde la demanda desborda la capacidad de atención con los recursos disponibles. De acuerdo con la revisión de la literatura, es un problema que apenas empieza a ser estudiado.

En Toro-Ocampo et al. (2016) se plantea un modelo matemático flexible con el que es posible resolver problemas de enrutamiento considerando flota propia y flota subcontratada con un depósito (VRPPC), considerando múltiples depósitos (MDVRPPC) y considerando la localización de los depósitos (CLRPPC). El modelo se basa en aprovechar la topología radial de la red de distribución y la adapta a este tipo de problemas los cuales involucran restricciones adicionales para garantizar la factibilidad de las respuestas halladas.

En Castaño et al. (2020) se presenta un modelo matemático para resolver el problema de ruteo verde usando flota propia y flota subcontratada, considerando múltiples depósitos (GMDVRPPC). El modelo planteado es del tipo bi-objetivo, donde se tienen en cuenta tanto los costos operativos como del impacto ambiental, medido a través del consumo de combustible de los vehículos. El estudio realiza un análisis de sensibilidad que considera la topografía del terreno (grados de inclinación) y estado de la vía para lo cual se calcula una distancia e inclinación virtual de los arcos. Para validar la propuesta, se usan instancias de la literatura especializada a fin de establecer el mejor modo de operación que relaciona el número de vehículos y el tiempo de entrega de acuerdo con la inclinación y estado de la vía.

\subsubsection{Problemas de enrutamiento sin retorno al depósito (open)}

Los problemas de enrutamiento abierto (OVRP) fueron inicialmente propuestos a inicios de la década de 1980 (Stenger et al., 2013; Samuel, 1983) cuando habían compañías que no tenían una flota de vehículos propia o era inadecuada para satisfacer la demanda total de sus clientes. Por lo tanto, los conductores no están obligados a volver al depósito después de realizar sus entregas. Por lo tanto, el objetivo de la OVRP es diseñar un conjunto de caminos hamiltonianos para satisfacer la demanda de los clientes.

Una generalización del OVRP se fundamenta en considerar que las rutas inicien desde diferentes depósitos 
y, una vez que han entregado las mercancías a los últimos clientes en sus rutas, no estén obligados a volver a los depósitos. Esta generalización es un problema combinatorio conocido como problema de enrutamiento abierto considerando múltiples depósitos (MDOVRP). Cuando se involucra adicionalmente el problema de localización de depósitos, aparece el problema de localización y enrutamiento abierto OLRP. Este tipo de problema está iniciando a ser discutido en la literatura especializada.

En la práctica, los problemas de enrutamiento abierto se presentan en situaciones como la entrega a domicilio de paquetes y periódicos, las rutas escolares, de personal empresarial, etc. (Braekers et al., 2016). Cuando se considera que los vehículos inician en diferentes depósitos y finalizan sus entregas en el último cliente visitado, esta generalización del OVRP se conoce como MDOVRP. En Tarantilis et al. (2005), se propone el MDOVRP por primera vez en el contexto de distribución de alimentos. Debido a que este problema es una generalización del OVRP, el MDOVRP es de tipo NP-duro, por lo tanto, los métodos heurísticos son comúnmente presentados en la literatura para encontrar soluciones de buena calidad de forma rápida y eficiente (Ho et al., 2008; Liu y Jiang, 2012; Dueck y Scheuer, 1990; Mirabi et al., 2010; Yao et al., 2014).

Liu y Jiang (2012) proponen un algoritmo genético híbrido para resolver el MDOVRP. Sin embargo, los autores proponen adicionalmente una formulación lineal entera mixta, la cual es la primera formulación matemática del modelo. La literatura sobre aproximaciones exactas para el MDOVRP es escasa y ha recibido poca atención por los investigadores. El más reciente método exacto reportado sobre MDOVRP es el presentado por Lalla-Ruiz et al. (2016) en el cual se presenta un modelo lineal entero mixto y se compara con Liu y Jiang (2012).

Cuando en el OVRP se considera la decisión adicional de elegir qué depósitos se deben abrir de un conjunto de depósitos disponibles, el problema resultante se conoce como OLRP. Este problema se ha introducido en Ho et al. (2008). Estos autores también han propuesto un modelo matemático exacto para resolver casos hasta de 50 clientes y 5 depósitos, cuyos resultados generan GAP con valores cercanos a 0. Además, se propone un algoritmo heurístico, basado en recocido simulado que consta de dos etapas. En primer lugar, una solución inicial se construye con un enfoque goloso y, en segundo lugar, la solución inicial se mejora con un procedimiento de recocido simulado con tres mecanismos de búsqueda: intercambio, inserción y movimientos 2-OPT. La propuesta se valida con los casos adaptados de Barreto et al. (2007), Prins et al. (2006) y Tuzun y Burke (1999).

En Granada et al. (2019) se plantea una formulación de programación lineal entera mixta donde se propone un conjunto de restricciones para obtener soluciones válidas, formadas por un gráfico que consiste en un árbol de expansión en cada componente conectado del gráfico. Este enfoque da como resultado una forma alternativa de evitar generar subtours, lo que simplifica significativamente el conjunto de restricciones asociadas con la conectividad de la solución y los requisitos de capacidad del vehículo. Los resultados computacionales muestran que la formulación propuesta es competitiva frente a los métodos más avanzados para este tipo de problemas. 


\subsection{Optimización multiobjetivo}

La optimización multiobjetivo es un proceso de optimización que implica el procesamiento simultáneo dos o más objetivos que necesariamente deben estar en conflicto y sujetos a un conjunto de restricciones. Para problemas no triviales multiobjetivo, no existe una única solución. En lugar de ello, hay un conjunto de soluciones, cuyo comportamiento reflejan el mejoramiento de un objetivo mientras los otros pueden mejorar o empeoran. Estas soluciones se denominan «soluciones óptimas de Pareto» 0 «Pareto eficientes». Encontrar un conjunto representativo y consistente de este tipo de soluciones es el objetivo que se persigue cuando se resuelve un problema de optimización multiobjetivo. La cuantificación de las ponderaciones en la satisfacción de los diferentes objetivos puede usarse como una metodología asociada para la construcción del conjunto de soluciones, pero deben asumirse criterios que favorezcan la propagación uniforme de los Paretos, que finalmente deberán representan los mejores compromisos posibles entre los diferentes objetivos.

Con el fin de emplear un concepto adecuado de solución óptima en un problema multiobjetivo, se define el óptimo de Pareto, a saber:

Sea $\Omega$ un conjunto no vacío de soluciones factibles y $\Psi(\cdot)$ un vector de funciones objetivo, una solución factible $x^{*} \in \Omega$ es llamada solución «Pareto óptima», si y solo si no existe ningún $x \in \Omega$ tal que $\Psi(x) \prec \Psi\left(x^{*}\right)$. La relación $(\cdot \prec \cdot)$ denota que cada coordenada del primer argumento es menor o igual que la correspondiente coordenada del segundo argumento y al menos una coordenada del primer argumento es estrictamente menor que la coordenada correspondiente del segundo argumento. Para resolver los problemas multiobjetivo, se pueden asumir valores para los parámetros del problema cuya solución conduce a una solución Pareto óptima. Para ampliar la discusión se puede consultar en Miettinen (1999); Ehrgott y Gandibleux (2003); Marler y Arora (2009).

Una metodología usada para los modelos de solución matemáticos exactos es la $\varepsilon$-constraint. Esta consiste en seleccionar un objetivo para ser optimizado mientras que los otros objetivos se transforman en restricciones asumiendo estos límites superiores. El planteamiento general es presentado en las ecuaciones 2.1 y 2.2, como sigue:

$$
\begin{gathered}
\min _{x} \Psi_{1}(x), \\
\Psi_{k}(x) \leq \varepsilon_{k} k=2, \ldots, m \\
x \in \Omega
\end{gathered}
$$


Cohon y Marks (1975) indican que el método $\varepsilon$-Constraint puede ser obtenido de las condiciones de optimalidad de Kuhn-Tucker para problemas de optimización multiobjetivo. Una variación sistemática de los parámetros $\epsilon_{k}$ puede conducir a soluciones Pareto óptimas (Marler y Arora, 2009). Si existe una solución $\varepsilon$-constraint, la formulación es débilmente Pareto-óptimo, como se plantea en Miettinen (1999). Sin embargo, si la solución es única entonces es Pareto-óptimo. Para más de dos objetivos, la formulación puede conducir a soluciones infactibles; la dificultad que se puede presentar está en la definición del valor $\epsilon_{k}$ ya que un valor no adecuado podría hacer que el algoritmo de solución no tenga convergencia o que encuentre de forma parcial las soluciones del Pareto-óptimo. Sin embargo, para dos objetivos, el método puede conducir a soluciones Pareto-óptimo con variaciones del $\epsilon$.

Para generar los puntos o soluciones que conforman del frente o frontera de Pareto, es necesario definir los valores de $\epsilon_{k}$ como pasos discretos; para esto, primero se optimiza con respecto a cada objetivo de forma individual y se consideran las restricciones originales. Esto genera los valores mínimos y máximos para cada objetivo. Los puntos intermedios del frente se obtienen variando $\epsilon$ dentro del rango mínimo y máximo.

\subsubsection{Dominancia}

Para la construcción de soluciones en algoritmos multiobjetivo, se hace necesario la definición de la dominancia que básicamente indica si una solución es mejor. Cuando una solución en comparación con otra es mejor, se le llama «solución dominada», el caso contrario es «no dominada».

El concepto de dominancia es definido por Deb (2014) de la siguiente forma:

Una solución $X_{1}$ domina a otra solución $X_{2}$ cuando las siguientes condiciones son verdaderas:

1. La solución $X_{1}$ no es peor que la solución $X_{2}$ en ninguno de los objetivos.

2. La solución $X_{1}$ es estrictamente mejor que la solución $X_{2}$ en, por lo menos, uno de los objetivos.

Si alguna de las condiciones no se cumple, se dice que la solución $X_{1}$ no domina la solución $X_{2}$.

En los problemas multiobjetivo, para la construcción de una frontera de Pareto, se definen regiones de región inferior, región de dominancia y región no inferior (Wu y Azarm, 2001). En la Figura nro. 3 se muestra la relación que tienen estas regiones con cada solución que compone un frente o frontera de Pareto para un problema de dos objetivos. La región delimitada por los puntos $\left[r_{1}, p_{f}, r_{2}, p_{g}\right]$ corresponde a la región inferior del punto $p_{f}$ (región que domina); la región de los puntos $\left[a_{2}, O, b_{1}, p_{f}\right]$ corresponde a la de dominancia del punto $p_{f}$. Las otras dos regiones restantes corresponden a las regiones no inferiores del punto $p_{f}$, allí es donde se ubicarán los puntos no dominados de $p_{f}$ y que generan el frente de Pareto. 


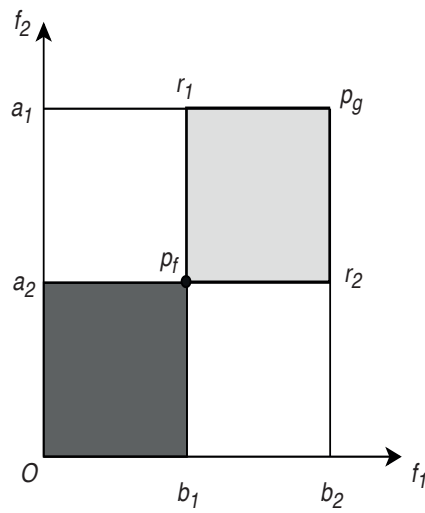

FiguRA NRo. 3. Regiones de dominancia. Elaboración propia.

\subsubsection{Métricas de frontera de Pareto}

En la solución de problemas multiobjetivos, son generados frentes o fronteras de Pareto. Este conjunto de soluciones representadas en el frente genera dificultad para compararse entre ellas. De allí nace la necesidad de utilizar indicadores que permitan comparar dichos frentes y que permitan determinar cuándo uno es mejor que otro. Es importante aclarar que las métricas de Pareto no se usan en este trabajo como parte del algoritmo de optimización en el principio de mejoramiento. Estas son usadas para comparar únicamente los resultados obtenidos. Las métricas de Pareto utilizadas en este trabajo son tomadas de la implementación realizada por Tian et al. (2017).

En este trabajo, las métricas de Pareto no son utilizadas en relación con la metaheurística. Estas son utilizadas únicamente para realizar la comparación entre frentes de Pareto, obtenidos después de la ejecución del algoritmo ILS.

\subsubsection{Solución de referencia}

En primera instancia, la solución de referencia adecuada para una métrica es el óptimo de Pareto. En el caso del problema del GVRPPCWE, no se tienen disponibles instancias con óptimos de Pareto obtenidos de modelos matemáticos exactos debido a que su planteamiento no tiene referencias en la literatura.

Para usar las métricas sin el óptimo de Pareto, se define un punto de referencia de menor calidad, lo que implica que para las métricas, su valor de mejoramiento sea inverso. En este trabajo, se define como punto de referencia el vector objetivo Nadir que corresponde a los límites superiores de los valores de las funciones objetivo de todos lo Paretos obtenidos (Deb, 2014). 


\subsubsection{Hipervolumen}

Este es uno de los indicadores usados más comúnmente como métrica de Pareto (Bradstreet, 2011). Un frente de Pareto es considerado como puntos en el espacio de soluciones, el hipervolumen es el espacio $n$-dimensional contenido entre otro frente y una referencia.

De acuerdo a la definición de hipervolumen, realizada por Bader y Zitzler (2011), donde se definen los conjuntos $A:\left\{f_{1}, f_{2}, \ldots, f(n)\right\}$ (que corresponde a todas las soluciones del Pareto aproximado) ${ }^{2}$ y $R$ : $\left\{r_{1}, r_{2}, \ldots, r(n)\right\}$ (que es definido como un vector con soluciones de referencia no-dominadas mutuamente). El conjunto $H(A, R)$ denota el subespacio que define el valor del hipervolumen. El conjunto $R$ será definido en $[1,1, \ldots, 1]$ debido a que el conjunto $A$ será normalizado en el rango $[0,1]$. En la Figura nro. 4 se muestra un ejemplo del volumen en tres dimensiones generado por un conjunto de cuatro puntos. En la Figura nro. 5 se muestra la representación de los conjuntos $A$ y $R$ en dos dimensiones y la relaciones que existen entre puntos las cuales permiten dividir el subespacio en fracciones más pequeñas que son usadas para la implementación de algoritmos eficientes en el cálculo del hipervolumen.
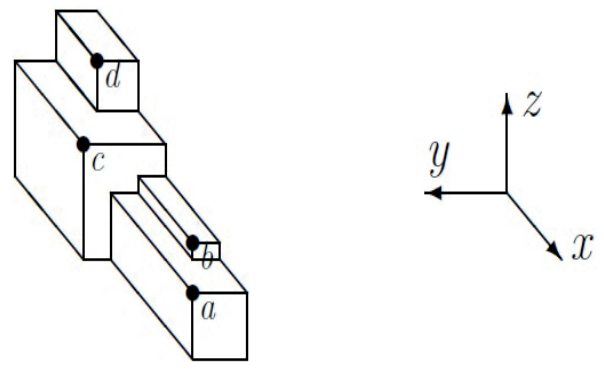

$\begin{array}{cccc} & x & y & z \\ & 11 & 4 & 4 \\ b & 9 & 2 & 5 \\ b & 5 & 6 & 7 \\ c & 3 & 3 & 10 \\ d & 3 & 3 & \end{array}$

FIgURA NRO. 4. Ejemplo hipervolumen en tres dimensiones. Cada punto genera un politopo. Tomado de: While et al. (2006) y Bradstreet (2011).

Para el cálculo del hipervolumen, si el problema es de menos de cuatro dimensiones, el valor es obtenido a través del método Hypervolume by Slicing Objetives (HSO). Este método consiste en seleccionar uno de los objetivos y generar cortes a lo largo de los puntos del frente. Esto se conoce como algoritmo de barrido dimensional. Los cortes eliminan a los puntos que son dominados. Lo anterior, reduce su complejidad computacional. Para dimensiones mayores a cuatro, donde el HSO no se comporta bien, es usada una estimación del hipervolumen a través de una simulación de Monte Carlo, el cual es un método aproximado que utiliza los pesos o contribuciones llamados «medidas

${ }^{2}$ El Pareto aproximado corresponde al frente que es dominado por el Pareto verdadero o óptimo global el cual es obtenido por los algoritmos de optimización aproximada. 


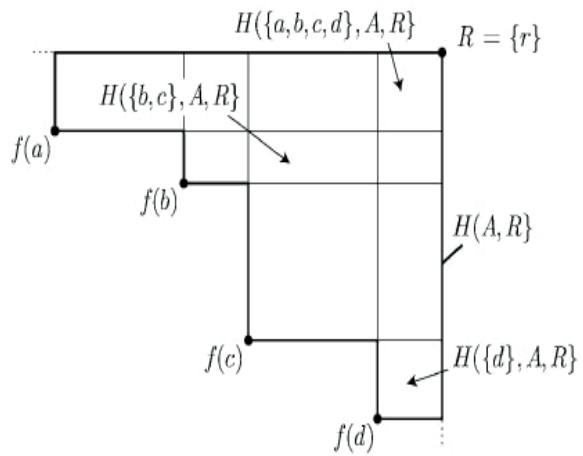

FiguRA NRO. 5. Representación de los conjuntos $A$ y $R$ en dos dimensiones y las relaciones entre puntos. Tomado de: Bader y Zitzler (2011).

de Lebesgue» de cada uno de los puntos en el hipervolumen total. Los pesos son calculados de forma aproximada haciendo un muestreo del subespacio del hipervolumen con áreas similares a las mostradas en la Figura nro. 5.

\subsubsection{Espaciado (Spacing)}

Esta métrica, tomada de Schott (1995), indica qué tan espaciadas se encuentran las soluciones de un frente de Pareto.

El valor es calculado midiendo la distancia de cada solución con respecto a sus vecinos. La formulación para esta métrica corresponde a la ecuación 2.3 .

$$
\text { Espaciado }=S=\left(\frac{1}{y-1} \sum_{i=1}^{y} d\left(V, y_{i}\right)^{2}\right)^{1 / 2}
$$

Donde, $d\left(V, y_{i}\right)$ denota la mínima distancia euclidiana de $y_{i}$ con el conjunto de soluciones $V$.

\subsubsection{Propagación (Spread)}

La métrica de propagación propuesta por Deb et al. (2002) está relacionada a la diversidad de los frentes de Pareto. Esta métrica es usada para calcular la uniformidad en la distribución del frente entre las soluciones extremas y es definida en 2.4 como:

$$
\Delta=\frac{d_{f}+d_{l}+\sum_{i=1}^{n-1}\left|d_{i}-\bar{d}\right|}{d_{f}+d_{l}+(N-1) \bar{d}}
$$


Donde, $d_{f}$ y $d_{l}$ corresponden a las distancias euclídeas entre cada punto extremo a la solución más cercana y $\bar{d}$ denota la distancia promedio entre todas las distancias $d_{i}$ del frente. $N$ denota el número de soluciones de Pareto.

En esta métrica, valores cercanos a cero indican que el frente esta uniformemente distribuido y con bajas o nulas concentraciones de puntos en el espacio de solución. Para problemas con más de dos objetivos, para que el valor de esta métrica sea cero, se tendrán que hallar todos los puntos que componen el hipervolumen del frente, lo que se considera complejo matemática y computacionalmente. También podrán existir casos en donde la distancia $d_{i}$ es muy variable por soluciones dispersas en el frente; en estos casos, la métrica podrá tener un valor mayor a uno.

\subsection{Enrutamiento de vehículos enfocado en logística de última milla}

La logística de última milla hace referencia a todos los procesos o actividades desarrolladas en la última etapa de logística para la distribución o entrega de productos al consumidor final. Esta contribuye al desarrollo dinámico de comercios locales y globales, y se enfoca siempre hacia el beneficio de las sociedades debido a la necesidad de movilizar bienes o servicios de un lugar a otro (Carranza-Huartos, 2019). En el último siglo, el problema de logística urbana ha destacado como el aspecto más relevante dentro del problema de última milla dado que los centros urbanos tienden a crecer y a consolidarse por antonomasia como los lugares de mayor crecimiento económico y de mayor actividad industrial los cuales envuelven la mayoría de actividades humanas y/o sociales (Jaimes et al., 2014). El $90 \%$ de los estudios realizados en la logística de última milla corresponden a la resolución de problemas de transporte, los cuales abordan, como variables de interés, los costos de viaje asociados a una red de suministros. Uno de los problemas que surgen al considerar como única variable de interés los costos de la red de suministros consiste en que se pierde de vista el comportamiento del cliente que, en términos generales, representa un factor sustancial para el estudio de la logística de las ciudades. Con lo anterior, estudios como los realizados a principio de los 70 por Tillman (1969), en el cual se introducen clientes estocásticos, permiten analizar una red de suministros para la minimización de costos de transporte pero considerando un comportamiento variable en la demanda del cliente.

Posteriormente, Dell'Amico y Hadjidimitriou (2012) hacen un estudio en el que no solo se consideran demandas estocásticas, sino también el hecho de que, durante el planeamiento de una red de suministros, se pueden presentar clientes difusos, asemejándose más a un problema de la vida real. Así, estudios en los cuales se involucre de forma dinámica el comportamiento de los clientes hacen del problema de logística de ciudades un tema de mayor preponderancia dentro de la cadena de suministros. Zhuge et al. (2019) propone un modelo de optimización para las demandas estocásticas para poder dar solución a la estabilidad de las rutas y de los horarios de los conductores de las entregas. El modelo se basa en el flujo de la red de productos múltiples el cual se utiliza para reducir al mínimo los costos de transporte y la desviación diaria de las trayectorias dependientes del día y de las zonas de la demanda. 
Adicionalmente, utilizan los métodos de relajación de Lagrange y el método de multiplicadores de dirección alterna para manejar las restricciones laterales complejas. Demuestran la eficiencia del método en una red de distribución de Beijing.

Debido a que la última milla es el eslabón final entre empresa y cliente, estudios hechos por Jaimes et al. (2014) permiten realizar un análisis de las principales tendencias y comportamientos de las compras para el hogar, centrándose principalmente en productos alimenticios. El estudio de los autores en cuestión se hace a través del uso de fuentes de información primarias y herramientas estadísticas sobre un centro urbano en Colombia, en el cual se obtienen resultados sobre tres variables de especial interés, a saber: productos, vehículos, frecuencia de compra relacionada al tipo de instalación para la adquisición de alimentos.

Un factor esencial frente a la logística de distribución de última milla es el comercio electrónico. Como se sabe, la logística urbana ha sido un campo de interés de muchos frentes de investigación de operaciones debido al alto impacto sobre las sociedades y los comercios del mundo. También, se sabe que muchos enfoques sobre transporte urbano siguen una tendencia en optimización combinatorial y que, de acuerdo a Perboli et al. (2018a), esta medida adoptada durante las últimas tres décadas ha venido presentando un par de debilidades que limitan la capacidad exploratoria del problema y compromete la incorporación de la tecnología a la industria. Los autores justifican que las dos debilidades propias del problema de transporte actual son: la limitación al tratamiento de instancias clásicas que a menudo no son creadas para aplicaciones urbanas, lo que crea un vacío en la validación de modelos que se puedan adaptar a casos de la vida real, y la inexistencia de una forma de estandarizar la mezcla de datos recopilados de diferentes fuentes con entornos reales, es decir, no es posible extraer información de diferentes fuentes que puedan adaptarse a datos reales.

En esa medida, la investigación planteada por los autores busca superar esas dos debilidades expuestas al proponer un marco de optimización simulada que permita construir instancias más reales y evaluar diferentes configuraciones operativas. Para ello, se realiza un estudio en la ciudad de Turín en el que se pretende evaluar el impacto de las opciones de entrega multimodal para satisfacer el e-commerce. Al igual que los autores anteriores, Zhou et al. (2018) presentan un nuevo problema de logística de ciudades que surge del comercio electrónico (ecommerce) en la distribución de última milla. El problema involucra dos niveles (Two-Echelon) de enrutamiento de vehículos, siendo el primer nivel un diseño de rutas para una flota de vehículos que empiezan desde unos centros de distribución, los cuales deben transportar las demandas de los clientes hasta un subconjunto de satélites o depósitos intermedios. El segundo nivel hace referencias al diseño de rutas óptimas para una flota de vehículos que empiezan en los satélites para atender a todos los clientes (MDVRP). El problema es resuelto mediante un algoritmo genético hibrido de múltiples poblaciones sobre el cual se usa una heurística eficaz para obtener soluciones iniciales de muy 
buena calidad. Se comprueba la efectividad del algoritmo sobre varias instancias de la literatura y sobre instancias del mundo real.

Por otro lado, Van Hentenryck et al. (2010) presentan un algoritmo de optimización hibrida-multietapa que considera una técnica exacta, programación de restricciones y búsqueda de vecindario grande. El algoritmo propuesto permite hacer un planeamiento estratégico, enfocado hacia el problema de asignación de productos básicos para la recuperación anti-desastres (SCAP), centrado en la distribución de última milla estocástica. Los SCAP son problemas de optimización estocástica complejos que combinan la asignación de recursos, el enrutamiento de almacenes (FLRP) y el enrutamiento en paralelo de vehículos (VRP). El algoritmo propuesto por los autores es validado ante escenarios de desastres por huracanes que son simulados en el Laboratorio Nacional de Los Álamos. Este se construye con el propósito de ayudar a las organizaciones federales de Estados Unidos. Posteriormente, y basados en el tema, Van Hentenryck et al. (2011) proponen método de solución para el problema de recuperación de desastres de última milla para la restauración de energía, es decir, un método que permite programar y asignar una flota óptima de vehículos para las restauración de una red de energía eléctrica en tiempo mínimo, después de un desastre. El método propuesto presenta un enfoque de cuatro etapas que se basan en la idea de inyección de restricciones, la cual desacopla la optimización de restauración de energía y el enrutamiento de vehículos, mientras se sigue conservando la idea de que aún se trabaja la parte de restauración en la etapa de enrutamiento. El método propuesto demuestra ser eficiente y con soluciones de muy buena calidad ante escalas grandes de desastres, basadas en la infraestructura de los Estados Unidos.

Dentro de la cadena de suministros y logística es bastante común encontrar sistemas (Multi-echelon) utilizados por administraciones públicas en sus estrategias de planificación de transporte y tráfico, y por empresas, que actualmente diseñan sus propios sistemas de distribución. En esa dirección, Perboli et al. (2011) proponen un VRP capacitado de dos niveles para el problema de logística de distribución de última milla. La investigación realizada tiene dos niveles. En el primero, se plantea una familia de problemas de enrutamiento de dos niveles (CVRP), en el cual se toma, como acción principal, gestionar un conjunto de rutas óptimas para una flota de vehículos que inicien en unos centros de distribución determinados; lo anterior, con el fin de llevar mercancías hasta unos depósitos intermedios, llamados satélites. En la segunda etapa, se diseña un VRP capacitado tradicional en el cual se planea un conjunto de rutas que inicien en los satélites para llevar la mercancía hasta los clientes. Para ello, los autores presentan un modelo exacto del VRP de dos niveles, algunas desigualdades válidas para ayudar a la relajación del problema y dos math-heurísticas basadas en dicho modelo. Los resultados demuestran ser promisorios y se prueban instancias de hasta 50 clientes. 
A pesar de que los enfoques previamente vistos de última milla se han basado en la minimización de costos de transporte, Boyer et al. (2009) presentan un estudio promisorio que examina el efecto sobre dos factores importantes en la eficiencia general de ruta de entrega como indicador de última milla (densidad cliente y longitud de ventana de entrega). Los resultados obtenidos proporcionan información para los gerentes de logística y marketing que deben equilibrar los deseos de los usurarios para una mayor comodidad en la eficiencia comercial de las empresas de los Estados Unidos, con enfoque en e-commerce. Aplicada al e-commerce se pueden encontrar tres modos de operación que son: la entrega a domicilio, las cajas de recepción o centros de recolección de mercancía (Facility Problem) y los centros de recolección y entrega de mercancía (Pickup and Delivery Problem), y cada uno de estos modos a asumir por la empresa encargada depende, en su mayoría, del tipo de usuario y el tipo de servicio como ya se ha mencionado.

Sin embargo, Wang et al. (2014) exploran la competitividad de los tres modos, bajo diferentes escenarios que impliquen una alta densidad de población. Cada modo de operación es modelado a través de diferentes esquemas de enrutamiento de vehículos; estos son resueltos con algoritmos genéticos. Sobre cada modo se calcula una base estructurada de costos y una eficiencia operativa. Los modos de recepción consideran variantes en la ubicación de la caja de recepción así: i) está instalada en el garaje o patio de la casa del cliente; ii) está equipada con un mecanismo de acoplamiento y se recuperará después de que se retiren las mercancías del interior; iii) está compartida, se instala cerca de los clientes para su uso compartido.

Los resultados obtenidos por los autores demuestran que cada modo de operación funciona bajo diferentes escenarios: los modos i) y ii) funcionan mejor en una zona con poca densidad poblacional y pocos pedidos, y el de tipo iii) funciona mejor en lugares con mayor concentración poblacional como las ciudades.

Por otro lado, Han et al. (2020) realizaron un estudio donde utilizan vehículos para transportar las mercancías y los drones son utilizados para ubicar a los clientes verticalmente. Lo anterior, lo desarrollan mediante un problema de enrutamiento de vehículos con tres objetivos a saber: minimizar el consumo total de energía de los vehículos, minimizar el consumo total de energía de los drones y el número total de los vehículos.

Así mismo, se tienen, en cuenta las ventanas de tiempo y las limitaciones de transporte de los drones. Para la solución del problema, diseñan un algoritmo novedoso de colonia de abejas exploradoras artificiales para mejorar las capacidades de búsqueda global, donde cada solución está representada por un vector bidimensional. Además, se incorpora el método de inicialización basado en la Heurística de Inserción por Empuje. Mientras que se utiliza una heurística mejorada para la realización de una búsqueda local para maximizar las capacidades de utilización.

Recientemente, Cai et al. (2018) proponen un algoritmo metaheurístico para resolver el problema de entrega en el último kilómetro (LMDP); el cual es una combinación del VRP capacitado, multidepósito, abierto y el problema de recogida y entrega con ventanas de tiempo (PDPTW). El algoritmo se basa en una búsqueda local iterada aplicando una búsqueda adaptativa de grandes vecindarios en la fase de perturbación para ampliar el alcance de esta. 


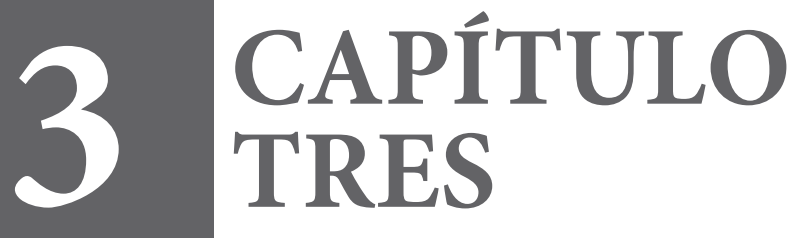




\section{MODELO MATEMÁTICO}

\subsection{Marco de referencia}

\subsubsection{Similitud de la operación en las redes de distribución de energía eléctrica y los problemas de enrutamiento de vehículos}

El modelo matemático que está siendo propuesto surge de comparar la generación de rutas para resolver los VRP con el problema de reconfiguración de las redes de distribución de energía eléctrica (Lavorato et al., 2012). Las redes de distribución de media tensión son operadas de manera radial; las cuales, haciendo uso de interruptores de apertura y cierre, cambian de una configuración a otra, manteniendo adecuados niveles operativos con la nueva topología radial. El objetivo de reconfigurar las redes de distribución de energía eléctrica radica en optimizar su operación. La solución de este problema consiste en determinar una topología radial que minimice las pérdidas, cumpliendo niveles de tensión, sin violar la capacidad de corriente en los circuitos. A pesar de lo anterior, se tienen algunas diferencias en la operación de las redes de energía eléctrica y las de transporte de carga, entre las que cabe destacar el grado de los nodos. En las redes de energía eléctrica se presentan nodos con grado superior a 2, como se observa en la Figura nro. 6, por lo que se genera una estructura tipo árbol; en las segundas, el grado de los nodos es menor o igual a 2, como se observa en la Figura nro. 7, lo que genera una estructura tipo arborescencia. Como se puede observar, se generan rutas que parten de un depósito y llegan a un nodo terminal.

Si se parte del modelo matemático, usado en la solución del problema de la operación de redes primarias de distribución, como se observa en la Figura nro. 6 y se adicionan ciertas restricciones al grado de los nodos, de menor o igual a dos, entonces se obtiene el modelo matemático para el problema de enrutamiento de vehículos abierto (open), tal como se observa en la Figura nro. 7. Con base en el enrutamiento anterior, al efectuar el retorno desde el último 
Introducción al problema de enrutamiento de vehículos en la logística de distribución

nodo hasta el de origen; son obtenidas trayectorias cerradas, como se muestra en la Figura nro. 8. Dado lo anterior, es propuesto un nuevo modelo matemático para ser usado como base en el planteamiento de variantes abiertos, cerrados y mixtos (rutas cerradas y abiertas) en la temática del VRP.

El modelo matemático es planteado en las ecuaciones 3.1 hasta la 3.11 y se describe como sigue: se cuenta con un conjunto de $j$ clientes que deberán ser atendidos, como máximo, con $k l$ vehículos homogéneos, que parten de un depósito único indexado con 0. De esta forma, el conjunto de todos los vértices es $V=I \cup J$. Con el fin de validar la no violación de la capacidad $Q$ del vehículo, se define la variable $t_{i j}$ con $i, j \in V$, con la cual se contabiliza la cantidad del producto que circulan entre los nodos $i$ y $j$; también se define un conjunto de distancias $c_{i j}$ con i, $j \in V$ y demandas $D_{j}$ con $j \in V$.

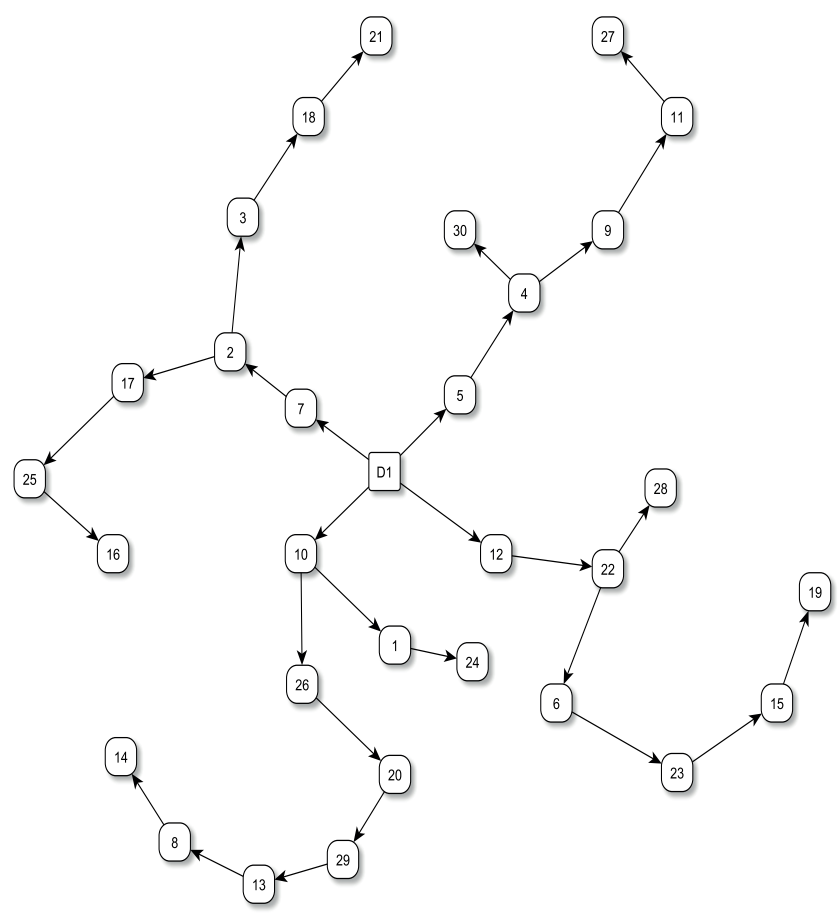

Figura NRO. 6. Topología de árbol.

Elaboración propia.

Las variables que usa el modelo se definen así: $x_{i j}$ identifican los arcos activos; $f_{i j}$ corresponde a la variable tipo-binaria la cual define si el consumidor que está localizado en el nodo $j \in J$ es atendido por la ruta que inicia en el centro de distribución $i \in I$. Por su parte, $a_{i j}$ correspondiente al arco de retorno al depósito desde el nodo 
terminal por cada ruta generada. La función objetivo 3.1 representa la minimización de los costos de las rutas. El nodo de demanda $j$ deberá tener un arco de llegada que lo conecta a la ruta y se asegura mediante 3.2. La ecuación 3.3 permite garantizar que todos los nodos tengan grado 2, es decir, que se ingrese y salga del nodo una única vez. La expresión 3.4 que corresponde al grado del depósito permite identificar e número de rutas que salen e ingresan al depósito. La expresión 3.5 hace referencia a una restricción asociada a la capacidad de depósito. La ecuación 3.6 asegura que un arco sea seleccionado en una sola dirección. Luego, la ecuación 3.7 representa el balance de flujo en cada nodo f. La ecuación 3.8 representa el grado de cardinalidad. La expresión 3.9 permite que los vehículos cumplan con la restricción de capacidad. La expresión 3.10 limita el número de rutas que puedan salir desde un depósito, de acuerdo a la capacidad del depósito y la de los vehículos. Finalmente, mediante la ecuación 3.11 se garantiza que la flota de vehículos sea suficiente para atender la demanda de los clientes.

Las topologías generadas por el uso del presente modelo matemático serán descritas en tres etapas, así: En la primera etapa, al considerar las restricciones 3.2, 3.5, 3.6, 3.7,3.8 y 3.9, se contempla la generación de una estructura tipo árbol, como el observado en la Figura nro. 6. En esta figura, el depósito es identificado con el nodo D1, el cual es conectado con cuatro arcos. En el grafo, los nodos 2, 4, 22, 10 son conectados a través de 3 arcos, el resto de nodos presentan 2 o menos conexiones. Un gráfico con las características descritas anteriormente, donde existen nodos con más de 2 conexiones, es denominado de tipo árbol.

$$
\min z=\sum_{\substack{i \in V \\ j \in J}} c_{i j} x_{i j}+\sum_{i \in J} c_{i 0} a_{i j}
$$

Sujeto a:

$$
\begin{array}{r}
\sum_{i \in V} x_{i j}=1, \quad \forall j \in J \\
\sum_{k \in J} x_{j k}+\sum_{i \in I} a_{i j}=\sum_{i \in V} x_{i j}, \quad \forall j \in J \\
\sum_{j \in J} x_{0 j}=\sum_{j \in J} a_{i j}, \quad \forall i \in I \\
\sum_{j \in J} t_{0 j}=\sum_{j \in J} D_{j}, \quad \\
\sum_{\substack{i \in V \\
i \neq j}} t_{i j}=\sum_{\substack{k \in V \\
k \neq j}}\left(t_{j k}+x_{j i} \leq 1, \quad \forall i, j \in V\right.
\end{array}
$$




$$
\begin{gathered}
\sum_{i, J \in V} x_{i j}=\operatorname{card}(J), \\
t_{i j} \geq Q x_{i j}, \quad \forall i, j \in V \\
\sum_{j \in J} x_{0 j} \leq k l \\
\sum_{j \in J} x_{0 j} \geq \sum_{j \in J} D_{j} / Q
\end{gathered}
$$

En la segunda etapa, son agregados, al modelo descrito anteriormente, las restricciones 3.3 y 3.4, además se asigna a la variable $a_{i j}=0$. Con estas consideraciones adicionales, es generado un grafo de tipo árbol, como se muestra en la Figura nro. 7.

En este grafo, todos los nodos, con excepción del nodo D1 (depósito), están conectados por 102 arcos. Este tipo de grafo es denominado de arborescencia, en el cual, las rutas parten de un depósito hasta llegar a un nodo final. De esta forma, son conformadas las rutas con un solo nodo terminal, similar al problema denominado OPEN.

En la tercera etapa, a la variable $a_{i j}$ se asigna un valor de 1 con el fin de obtener rutas cerradas, tal como se muestra en la Figura nro.8, en donde los arcos punteados representan el enlace que conecta al cliente final con el depósito del cual parte la ruta.

En este grafo se puede observar que las rutas inician y regresan al mismo depósito. A excepción del nodo depósito, todos los demás nodos presentan un grado 2, es decir, se conectan a través de dos arcos, uno de entrada y otro de salida.

Como fue planteado anteriormente, el modelo matemático descrito permite el desarrollo de algunas de las variantes de los problemas de enrutamiento de vehículos, los cuales consideran o no el retorno al depósito. Dichos problemas pueden ser planteados de forma separada o integrada. El primero representa las versiones abiertas (open), en donde la ruta inicia en el depósito y termina en el último cliente que es atendido; el segundo, las versiones clásicas, en el cual las rutas inician y terminan en el depósito; la tercera alternativa es una forma híbrida respecto a las dos anteriores, en donde se considera el enrutamiento de vehículos con y sin retorno al depósito. En estos casos, se analiza el uso combinado de flota propia y subcontrata. Adicionalmente, pueden ser planteadas algunas variantes con múltiples depósitos, como es el caso del MDVRP y CLRP. Otra característica de interés es la flexibilidad del modelo matemático cuando es usado en el estudio de problemas mono- y multiobjetivo.

Una de las características relevantes de este modelo es el hecho de que las alternativas de solución generan grafos conectados, es decir, que no se presentan nodos o depósitos aislados. De esta forma, la generación de las topologías de este tipo reemplazan las de eliminación de sub-tours denominadas MTZ (Miller et al., 1960).

Con base en los resultados obtenidos, se ha llevado a cabo un análisis computacional entre el modelo radial 
y el MTZ para los problemas VRP, MDVRP y CLRP. Gracias a dicho análisis, se pudo observar que el modelo-tipo radial presenta un mejor desempeño en todos los casos que fueron estudiados. $\mathrm{Al}$ analizar algunos casos de prueba de mayor complejidad matemática, se observa un mejor desempeño del modelo propuesto respecto al modelo MTZ. También se pudo concluir que el modelo radial está en capacidad de alcanzar soluciones logradas con métodos exactos para instancias de pequeña y mediana complejidad matemática, logrando llegar a GAP cero. Asimismo, se observan soluciones de excelente calidad cuando es usada la versión open, con instancias de alta complejidad matemática. Se vislumbra como el modelo radial planteado presenta una excelente capacidad de exploración en el árbol de búsqueda respecto al mismo tiempo computacional del modelo MTZ.

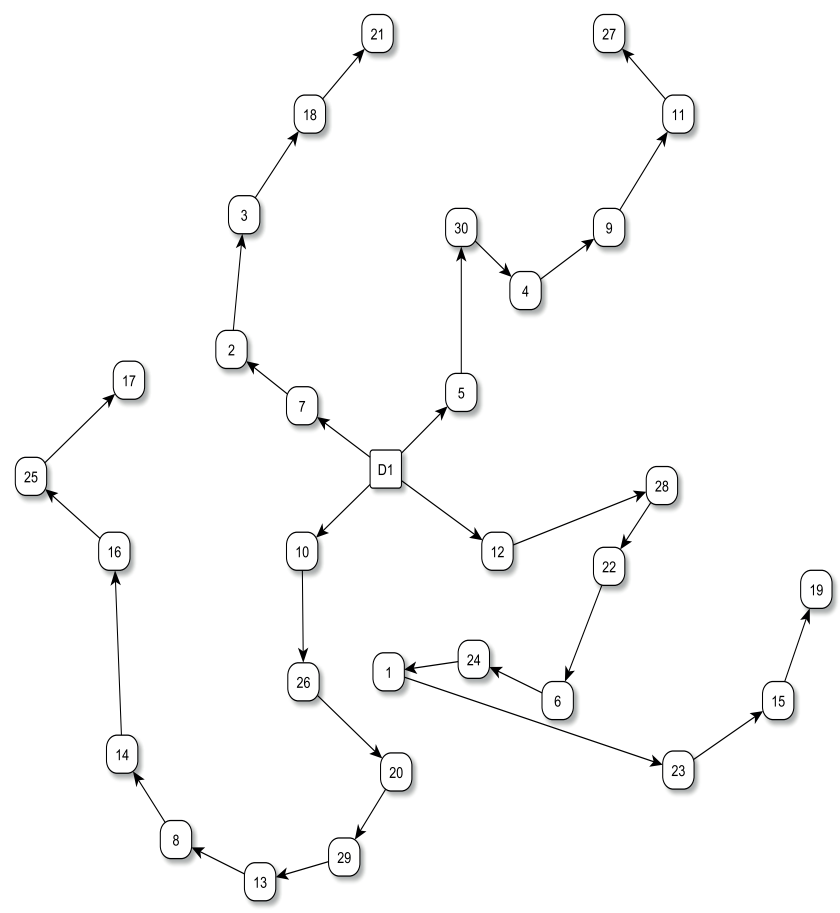

Figura NRO. 7. Arborescencia.

Elaboración propia. 


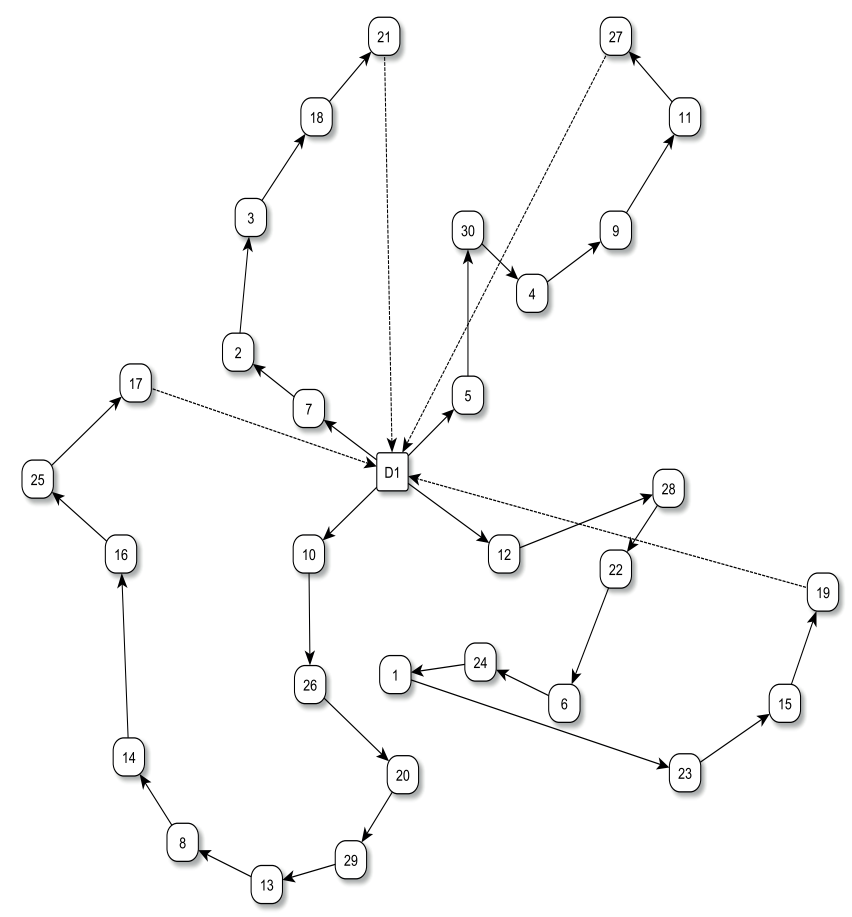

FIGURA NRO. 8. Rutas cerradas al depósito. Elaboración propia.

\subsubsection{Modelo matemático a ser propuesto}

Con base en el modelo matemático descrito, el cual se aplica en el estudio de la operación de redes de distribución de energía eléctrica; se propone un nuevo modelo matemático que resuelve diferentes variantes del problema de enrutamiento de vehículos. Para tal fin, se plantea un modelo general donde se considera la utilización de flota propia y subcontratada por lo que el model considera rutas abiertas y cerradas, denominado CLRPPC. A partir de este modelo, es posible obtener diversas versiones del problema de enrutamiento de vehículos, como son las versiones clásicas o denominadas cerradas, las versiones open; y las versiones híbridas cerradas y abiertas de los problemas CVRP, MDVRP y CLRP. Dicho modelo también es fácilmente adaptado en la solución de problemas de 2 o 3 objetivos, como son los de impacto ambiental y problemas de sostenibilidad. 

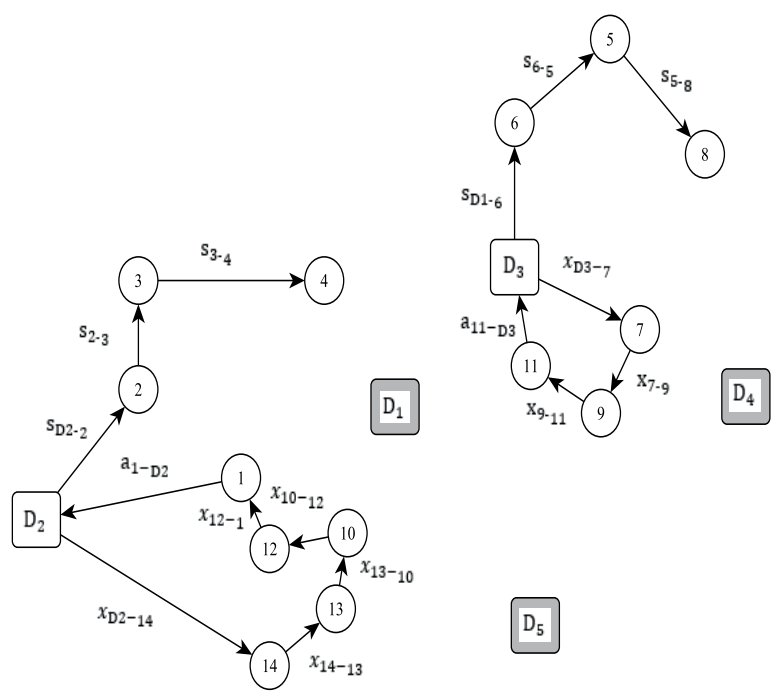

Figura NRO. 9. CLRPPC.

Elaboración propia.

\subsection{Problema de enrutamiento con flota propia y subcontratada}

\subsubsection{Problema de localización y enrutamiento con flota propia y subcontratada (CLRPPC)}

El problema de localización y enrutamiento incluyendo restricciones de capacidad servida con flota propia y flota subcontratada es un área considerada como una nueva línea de investigación (Prodhon y Prins, 2014). Esta situación se presenta cuando el nivel de actividades se incrementa, como es el caso de la industria de servicios; donde aparecen, de forma repetitiva, actividades como la reparación y el mantenimiento de la flota de vehículos. Por lo cual la flota es insuficiente para atender la demanda de los clientes. Otra situación que también presenta inconvenientes para la atención de la demanda es el caso de la logística de desastres. Toda la demanda sobrepasa la capacidad de atención con los recursos disponibles. Después de realizar una revisión de dicho problema en la literatura se concluye que es un problema poco estudiado.

En el problema de localización y enrutamiento considerando restricciones de capacidad (CLRP), se plantea la selección de depósitos y rutas para atender un conjunto de clientes, de tal manera que se obtenga el mínimo costo 
posible. Una variante del problema anterior considera la posibilidad de subcontratar la operación de algunas (o todas) las rutas. Este problema aparece cuando la flota propia es insuficiente para atender la demanda o una parte de los vehículos de la flota propia debe atender actividades de mantenimiento y reparación. El objetivo del CLRPPC es optimizar los costos de apertura de depósitos, los costos de los vehículos propios, así como los costos variables que estan asociados a la utilización de las rutas recorridas por la flota propia y subcontratada.

\section{Nomenclatura}

La nomenclatura del problema CLRPPC es la siguiente:

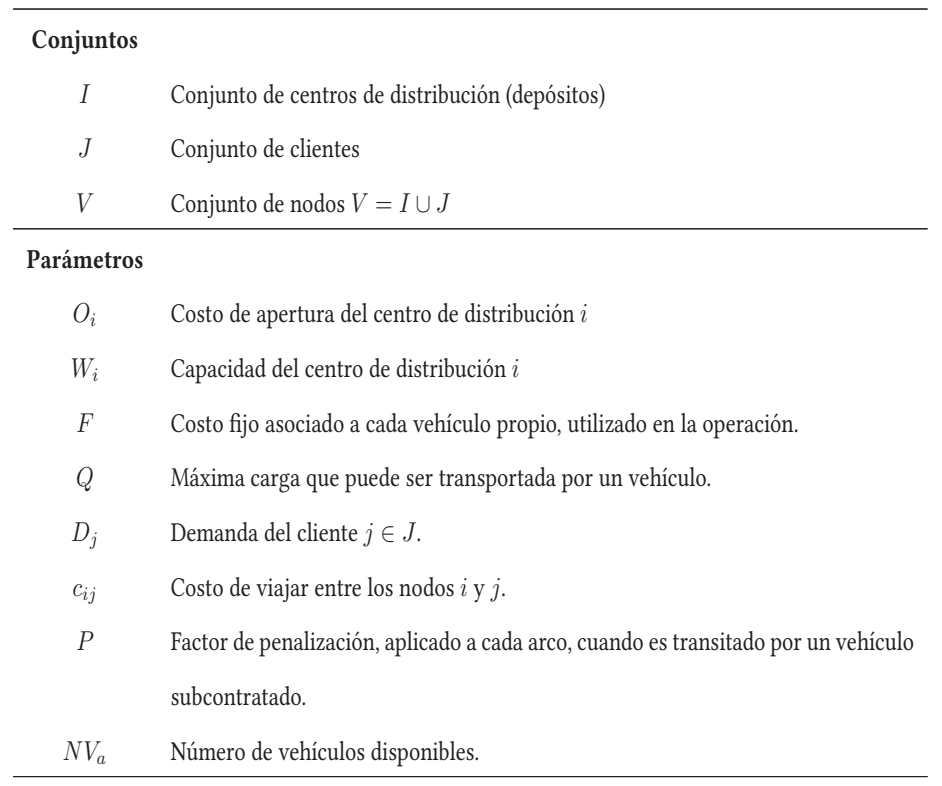

\section{Variables}

$x_{i j} \quad$ Variable binaria que es habilitada cuando el camino entre los nodos $i, j \in V$ es recorrido por un vehículo propio.

$s_{i j} \quad$ Variable binaria que es habilitada cuando el camino entre los nodos $i, j \in V$ es recorrido por un vehículo sucontratado.

$y_{i} \quad$ Variable binaria que indica la apertura del centro de distribución $i \in I$.

$f_{i j} \quad$ Variable binaria que define si el cliente ubicado en el nodo $j \in J$ es atendido por una ruta que inicia en el centro de distribución $i \in I$. 
$z_{j} \quad$ Variable binaria que determina si el cliente ubicado en el nodo $j \in J$ es el último de la ruta en ser atendido.

$a_{i j} \quad$ Variable binaria que indica si el vehículo usa el camino desde el nodo $j$ al centro de distribución, que está localizado en el nodo $i$.

$t_{i j} \quad$ Variable continua que indica la cantidad de carga transportada entre los nodos $i \mathrm{y}$ $j$ y recorrido por la flota propia.

$l_{i j} \quad$ Variable continua que indica la cantidad de carga transportada entre los nodos $i \mathrm{y}$ $j$ y recorrido por la flota subcontratada.

TABLA NRo. 3. Nomenclatura del problema CLRPPC. Elaboración propia.

El problema de localización y enrutamiento que usa flota propia y subcontratada (CLRPPC) se plantea como un problema lineal entero mixto y definido por las ecuaciones 3.12 hasta la 3.39. Este problema es representado en la Figura nro. 9. Como puede apreciarse en esta figura, de 5 depósitos que han sido propuestos, 2 fueron seleccionados. Además, puede observarse que hay rutas con retorno al depósito que corresponden a las rutas que efectúan los vehículos propios y otras que terminan en el último cliente que es servido y que responden al servicio tercerizado. Cada una de estas rutas, propias y tercerizadas, son identificadas por un gráfico completo. Las primeras, las rutas propias, son identificadas con la letra $x$, y la segundas, las rutas tercerizadas, son identificadas con la letra $s$. Este problema es considerado de gran complejidad matemática (NP-Duro) y es muy difícil de resolver.

$$
\min z=\sum_{i \in I} o_{i} y_{i}+\sum_{\substack{i \in I \\ j}} F a_{i j}+\sum_{i, j \in V} c_{i j} x_{i j}+\sum_{\substack{i \in I \\ j \in J}} c_{i j} a_{i j}+P \sum_{\substack{i \in V \\ j \in V}} c_{i j} s_{i j}
$$

Sujeto a:

$$
\begin{array}{r}
\sum_{i \in V} x_{i j}+\sum_{i \in V} s_{i j}=1, \quad \forall j \in J \\
\sum_{k \in J} x_{j k}+\sum_{i \in I} a_{i j}=\sum_{i \in V} x_{i j}, \quad \forall j \in J \\
\sum_{j \in J} x_{i j}=\sum_{j \in J} a_{i j}, \quad \forall i \in I
\end{array}
$$


Introducción al problema de enrutamiento de vehículos en la logística de distribución

$$
\begin{aligned}
& \sum_{k \in J} s_{j k} \leq \sum_{i \in V} s_{i j}, \quad \forall j \in J \\
& x_{i j}+x_{j i}+s_{i j}+s_{j i} \leq 1, \quad \forall i, j \in V \\
& \sum_{\substack{i \in V \\
i \neq j}} t_{i j}+l_{i j}=\sum_{\substack{k \in V \\
k \neq j}}\left(t_{j k}+l_{j k}\right)+D_{j}, \quad \forall j \in J \\
& \sum_{\substack{i \in V \\
j \in V}} x_{i j}+s_{i j}=\operatorname{card}(J), \quad \forall j \in J \\
& \sum_{i \in I} f_{i j} \leq 1, \quad \forall j \in J \\
& t_{i j} \leq Q x_{i j}, \quad \forall i, j \in V \\
& l_{i j} \leq Q S_{i j}, \quad \forall i, j \in V \\
& \sum_{j \in J} t_{i j}+l_{i j} \leq w_{i} y_{i}, \quad \forall i \in I \\
& \sum_{i \in V} s_{i j}+\sum_{k \in V} x_{j k}=1-z_{j}, \quad \forall j \in J \\
& 1+a_{i j} \geq f_{i j}+z_{j}, \quad \forall i \in I, \forall j \in J \\
& -\left(1-x_{j u}-x_{u j}\right) \leq f_{i j}-f_{i u}, \quad \forall i \in I, \forall j, u \in V \\
& f_{i j}-f_{i u} \leq\left(1-x_{j u}-x_{u j}\right), \quad \forall i \in I, \forall j, u \in V \\
& f_{i j} \geq x_{i j}, \quad \forall i \in I, \forall j \in J \\
& \sum_{i \in I} y_{i} \geq \sum_{j \in J} D_{j} / \sum_{i \in I} w_{i}, \quad \forall i \in I \\
& \sum_{j \in J} x_{i j}+s_{i j} \leq \sum_{j \in J} D_{j} / Q, \\
& \sum_{\substack{i \in I \\
j \in J}} a_{i j} \leq N V_{a}, \\
& x_{i j} \in\{0,1\}, \quad \forall i, j \in V \\
& s_{i j} \in\{0,1\}, \quad \forall i, j \in V \\
& y_{i} \in\{0,1\}, \quad \forall i \in I \\
& f_{i j} \in\{0,1\}, \quad \forall i \in I, \forall j \in V \\
& z_{j} \in\{0,1\}, \quad \forall j \in J \\
& a_{i j} \in\{0,1\}, \quad \forall i \in I, \forall j \in J
\end{aligned}
$$




$$
\begin{aligned}
& t_{i j} \in R, \quad \forall i, j \in V \\
& l_{i j} \in R, \quad \forall i, j \in V
\end{aligned}
$$

La función objetivo, representada por la ecuación 3.12, minimiza los costos de operación, correspondientes a la suma de los costos de apertura de centros de distribución de carga, los costos de apertura de las rutas y los costos variables por el uso de los arcos, ya sea por la flota propia o por la subcontratada.

Un nodo de demanda j está conectado a un arco de llegada con lo cual queda vinculada a una ruta que puede ser recorrida por la flota propia o por la subcontratada. Esto se representa en 3.13. Por su parte, 3.14 establece que la suma de los arcos de salida de un nodo de demanda es igual a la suma de los arcos de entrada. Este arco puede corresponder a la ruta propia $x$ o puede ser el retorno al centro de distribución de carga $a$; es decir, si se llega a un nodo con una ruta propia, entonces se sale con el mismo tipo de ruta. Así, 3.15 garantiza que, para un centro de distribución de carga $i$, el número de arcos de salida $x$ sea igual que el número de arcos de llegada $a$. 3.16 establece que, si se sale de un nodo con una ruta subcontratada, se deberá llegar con una ruta subcontratada. Es posible que se llegue al nodo pero no se salga de este (este caso corresponde a un nodo terminal de la ruta subcontratada). 3.17 no permite que un arco sea duplicado, definiendo de esta forma la orientación de un arco, es decir, solo se acepta un sentido, de $i$ para $j$ o de $j$ para $i$. La ecuación 3.18 representa el balance de flujos en los nodos tanto para las rutas propias como para las subcontratadas 3.19 relaciona los arcos activos que generan topologías radiales 3.20 garantiza que los nodos de demanda de una ruta estén conectados a un centro de distribución. 3.21 establece un límite de flujo por una ruta propia con base en la capacidad de los vehículos y la variable que establece el uso de este tipo de ruta. La ecuación 3.22 limita el flujo por una ruta subcontratada con base en la capacidad de los vehículos y la variable que establece el uso de este tipo de ruta. 3.23 limita el flujo que sale de un centro de distribución de carga con base en su capacidad y la decisión de construir ese centro de distribución de carga. En 3.24 identifica un nodo terminal para rutas propias (con un valor de $z=1$ ) y luego conecta este con el centro de distribución de carga. Si no existen arcos de salida para ese nodo de demanda es porque este corresponde a una ruta subcontratada. Si el nodo $j$ corresponde a un nodo terminal, entonces la ecuación 3.25 genera un arco de retorno. En las figuras nro. 10 y 11 se observa el comportamiento de la variable $z$ de acuerdo con los valores calculados.

Las ecuaciones 3.26 y 3.27, aplicadas a arcos activos propios, permiten identificar el nodo de inicio de la ruta propia y, de esta forma, posibilitan activar el arco de retorno al centro de distribución de carga. Si el arco entre el centro de distribución de carga $i$ y la demanda $j$ está activa, se debe garantizar que el nodo $j$ esté conectado con el centro de distribución de carga $i f_{i j}=1$, lo cual esta expresado en 3.28 .

La ecuación 3.29 establece un límite inferior para el número de centros de distribución de carga, lo cual es 
Introducción al problema de enrutamiento de vehículos en la logística de distribución

calculado con base en la suma de las demandas y la capacidad de dichos centros. La ecuación 3.30 permite garantizar que el número de rutas sea suficiente para atender la demanda. La ecuación 3.31 determina el máximo número de rutas atendidas por la flota propia. De estas índole, las ecuaciones 3.32, 3.33, 3.34, 3.35, 3.36 y 3.37 definen la naturaleza binaria de las variables $x_{i j}, s_{i j}, y_{i}, f_{i j}, z_{j}$ y $a_{i j}$. Finalmente, las ecuaciones 3.38 y 3.39 establecen las variables $t_{i j}$ y $l_{i j}$ como continuas. Estas representan la cantidad de flujos de carga que circula entre el nodo $i$ y el nodo $j$ por las rutas propias o subcontratadas, respectivamente.

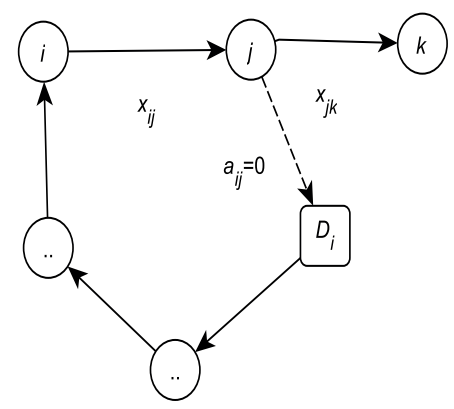

FIgURA NRo. 10. j no es nodo terminal. ${ }^{1}$

Elaboración propia.

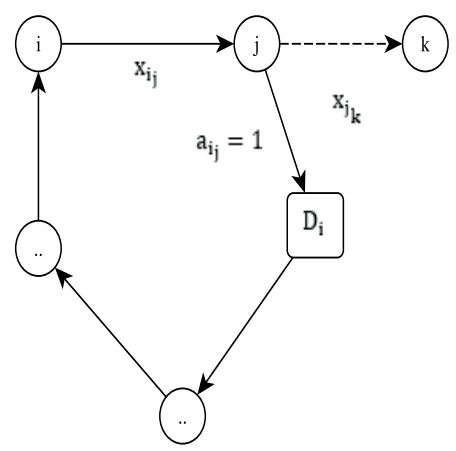

FigURA NRo. 11. jes nodo terminal. ${ }^{2}$ Elaboración propia.

${ }^{1} \mathrm{Si} z=0$, se establece que el nodo $\mathrm{j}$ no es terminal.

${ }^{2} \mathrm{Si} z=1$, se establece que el nodo visitado es terminal. 


\subsubsection{Problema multidepósito considerando flota propia y subcontratada (MDVRPPC)}

El modelo definido desde las ecuaciones 3.13 hasta la ecuación 3.41, es modificado para obtener el modelo del CVRPPC que se obtiene al definir I como un conjunto de un solo elemento, asociado a un único depósito. Este se consigue fijando $k$ con el valor de 1; adicionalmente en esta variante, a los parámetros $O_{i}$ y $F$ se les asigna un valor de 0 . Para los casos en los que se desee resolver el MDVRPPC, se requiere adicionar la restricción 3.41 en la que se indican los depósitos que están abiertos esto se logra al asignar un valor a $k$ correspondiente al número de depósitos abiertos. En la Figura nro. 12 se puede observar la representación del problema MDVRPPC, en la que el número de depósitos es un parámetro de entrada. Así como en el caso anterior, el problema está conformado por rutas cerradas y abiertas.

El modelo matemático que describe el problema MDVRPPC se define como:

$$
\min z=\sum_{i, j \in V} c_{i j} x_{i j}+\sum_{\substack{i \in I \\ j \in J}} c_{i j} a_{i j}+P \sum_{\substack{i \in V \\ j \in V}} c_{i j} s_{i j}
$$

Este se encuentra sujeto a las ecuaciones comprendidas entre (3.13) y (3.39).

$$
\sum_{i \in I} y_{i}=k, \quad \forall i \in I
$$

\subsubsection{Problema de enrutamiento con flota propia y subcontratada (VRPPC)}

El modelo matemático que describe este problema está definido por un único depósito, asociado a $i=1$ y $k=1$ con respecto al modelo del MDVRPPC desde el cual es atendido un conjunto de clientes $J$. Tal como se observa en la Figura nro. 13, las rutas inician en un mismo y único depósito. Así como en los casos anteriores, se observa una combinación de rutas cerradas y abiertas, vinculadas a los dos tipos de flota utilizadas.

$$
\min z=\sum_{i, j \in V} c_{i j} x_{i j}+\sum_{\substack{i=1 \\ j \in J}} c_{i j} a_{i j}+P \sum_{\substack{i \in V \\ j \in V}} c_{i j} s_{i j}
$$

Esta ecuación 3.42 se encuentra sujeta a las ecuaciones 3.13 y 3.39 . 

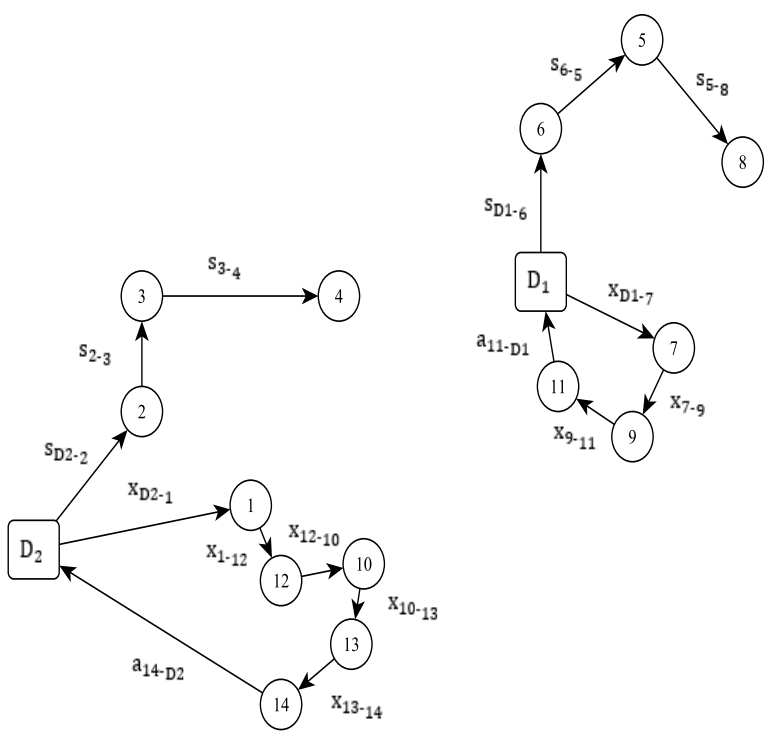

FigurA NRO. 12. MDVRPPC.

Elaboración propia.

\subsection{Problema de enrutamiento abierto}

En esta variante, se inicia la ruta en un depósito y se finaliza en el último cliente servido. También puede considerarse la ruta en reversa, es decir, la ruta puede iniciar en un cliente y finalizar en un depósito.

\subsubsection{Problema de localización y enrutamiento abierto (OLRP)}

El problema de localización y enrutamiento, con restricciones de capacidad y sin que los vehículos tengan que retornar al depósito de inicio (OLRP), es definido por un conjunto de depósitos $I$ que son propuestos para ser abiertos, desde los cuales es atendido un conjunto de clientes $J$. Este problema considera restricciones de capacidad tanto para los depósitos como para los vehículos. La flota de vehículos es ilimitada. En la Figura nro. 14, se observa que, de 5 depósitos propuestos, 2 son seleccionados en la configuración final, también en la figura, se observan 4 trayectorias abiertas, las cuales parten de los depósitos. El modelo usa un grafo completo, el cual es identificado con la variable $s$. 


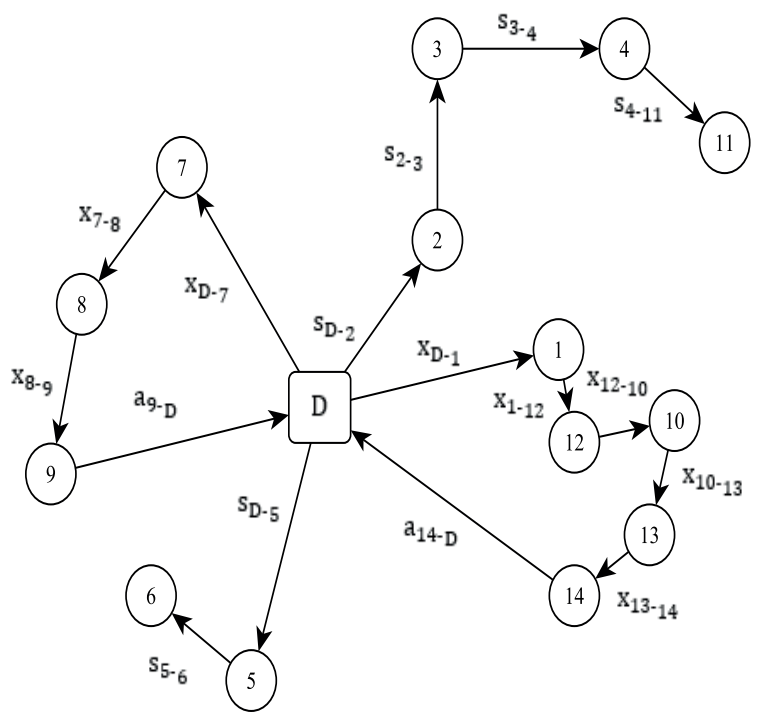

FIGURA NRO. 13. VRPPC.

Elaboración propia.
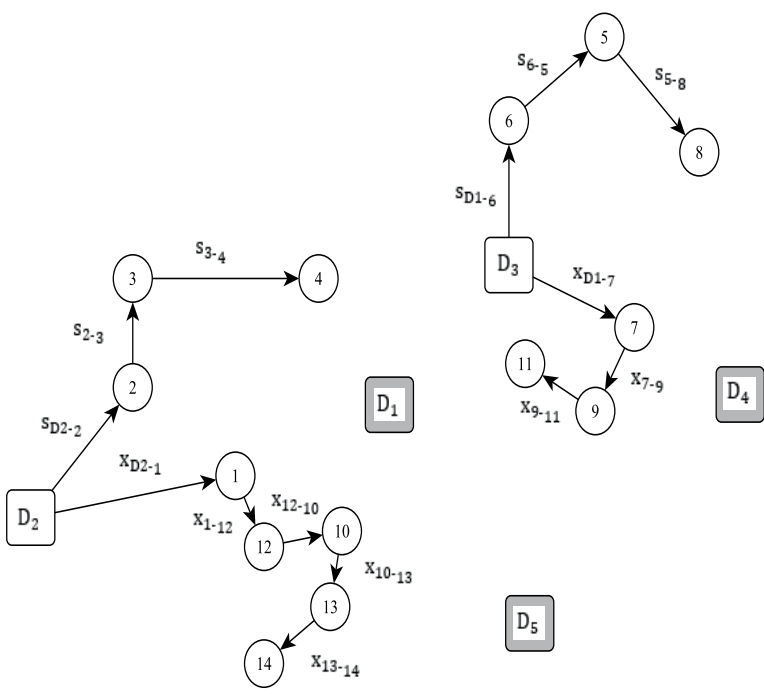

FIGURA NRO. 14. OLRP.

Elaboración propia. 


$$
\operatorname{mín}=\sum_{i \in I} o_{i} y_{i}+\sum_{\substack{i \in I \\ j \in J}} F s_{i j}+\sum_{\substack{i \in V \\ j \in V}} c_{i j} s_{i j}
$$

Esta ecuación 3.43 está sujeta a las ecuaciones 3.13 hasta la 3.39 .

$$
x_{i j}=0 \quad \forall i, j \in V
$$

$$
t_{i j}=0 \quad \forall i, j \in V
$$

\subsubsection{Problema de enrutamiento multidepósito abierto (MDOVRP)}

Para esta variante del problema de enrutamiento abierto, la ubicación de los depósitos corresponde a una información conocida, como se puede observar en la Figura nro. 15.

En este problema, el número de depósitos es un parámetro de entrada, como se observa en la ecuación 3.41 . El valor de $k$ es fijado al inicio del proceso. A partir de los depósitos que han sido fijados, se identifican las trayectorias abiertas, que inician en un depósito y finalizan con el último cliente que es servido. El modelo usa un grafo completo, el cual es identificado por los arcos $s_{i j}$.

$$
\min z=\sum_{\substack{i \in V \\ j \in V}} c_{i j} s_{i j}
$$

Esta ecuación 3.46 se encuentra sujeta a las ecuaciones desde 3.13 a 3.40, y las ecuaciones 3.44 y 3.45 .

\subsubsection{Problema de enrutamiento abierto con único depósito (OVRP)}

Este problema considera un único depósito, como puede ser observado en la Figura nro. 16. El modelo matemático para resolver este problema es similar al del MDOVRP con una sola diferencia, la cual asigna un valor de 1 al conjunto de depósitos. Para este caso, se generan una serie de trayectorias abiertas que inician en el depósito y finalizan en el último cliente que es servido. El modelo emplea un grafo completo, el cual es identificado con la variable $s_{i j}$. 

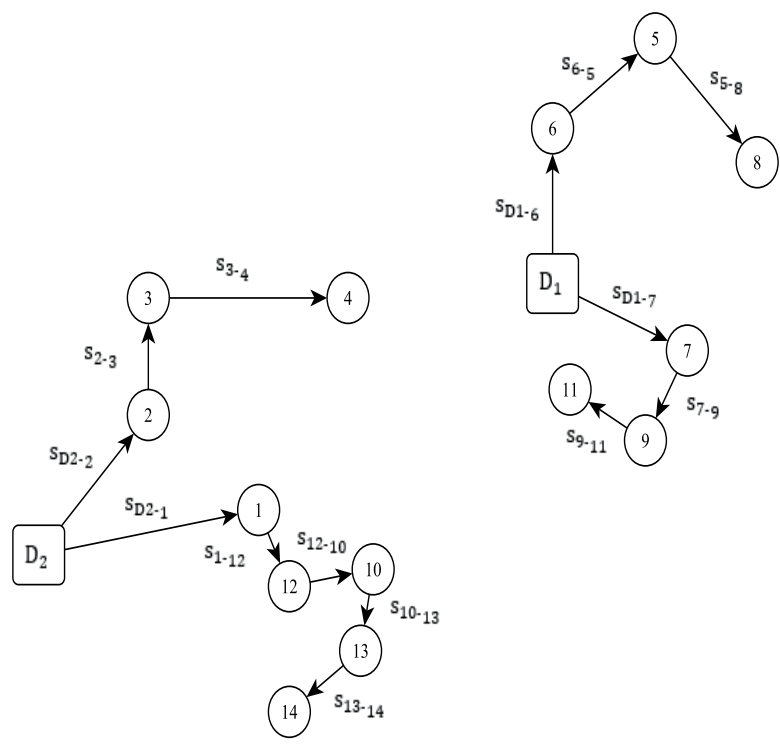

FIGURA NRO. 15. MDOVRP.

Elaboración propia.

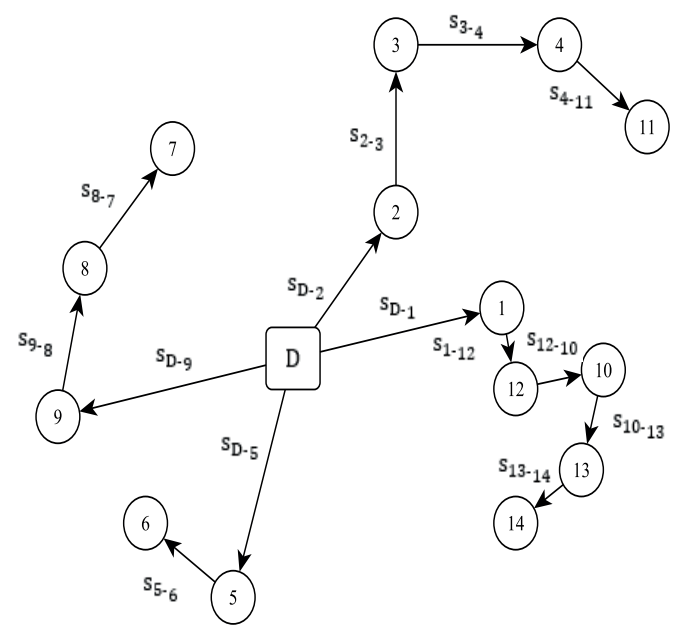

FIGURA NRO. 16. OVRP.

Elaboración propia. 


\subsection{Problemas de enrutamiento de vehículos estándar}

\subsubsection{Problema de localización y enrutamiento estándar (CLRP)}

Para la solución de este problema, el modelo deberá seleccionar los depósitos a abrir de un conjunto de depósitos disponibles además de lo anterior, se deben generar un conjunto de rutas, las cuales deben satisfacer completamente la demanda en los nodos, tal como se observa en la Figura nro. 17. La versión planteada asume trayectorias cerradas. En el ejemplo, son propuestos 5 depósitos, de los cuales 2 son seleccionados. El modelo usa un grafo completo que es identificado con la variable $x_{i j}$. El último arco, identificado con la variable $a_{i j}$, se encarga de cerrar la trayectoria, permitiendo el retorno al depósito del cual inicia.

$$
\min z=\sum_{i \in I} o_{i} y_{i}+\sum_{\substack{i \in I \\ j \in J}} F a_{i j}+\sum_{i, j \in V} c_{i j} x_{i j}+\sum_{\substack{i \in I \\ j \in J}} c_{i j} a_{i j}
$$

La anterior ecuación se encuentra sujeta a las ecuaciones 3.13 hasta la 3.39 .

$$
\begin{aligned}
& s_{i j}=0 \quad \forall i, j \in V \\
& l_{i j}=0 \quad \forall i, j \in V
\end{aligned}
$$

\subsubsection{Problema de enrutamiento multidepósito estándar (MDVRP)}

Este problema considera múltiples depósitos de carga con capacidad limitada, desde los cuales se debe atender un conjunto de nodos $J$ con una flota ilimitada de vehículos. Una representación de este problema puede verse en la Figura nro. 18. El número de depósitos de carga se considera como parámetro de entrada. El modelo usa un grafo completo y sus variables son identificadas por $x_{i j}$, la variable $a_{i j}$ identifica la conexión que existe entre el último nodo y el depósito de carga.

$$
\min z=\sum_{i, j \in V} c_{i j} x_{i j}+\sum_{\substack{i \in I \\ j \in J}} c_{i j} a_{i j}
$$

Donde 3.50 se encuentra sujeta a las ecuaciones desde 3.13 a 3.39, y las ecuaciones 3.48 y 3.49 . 

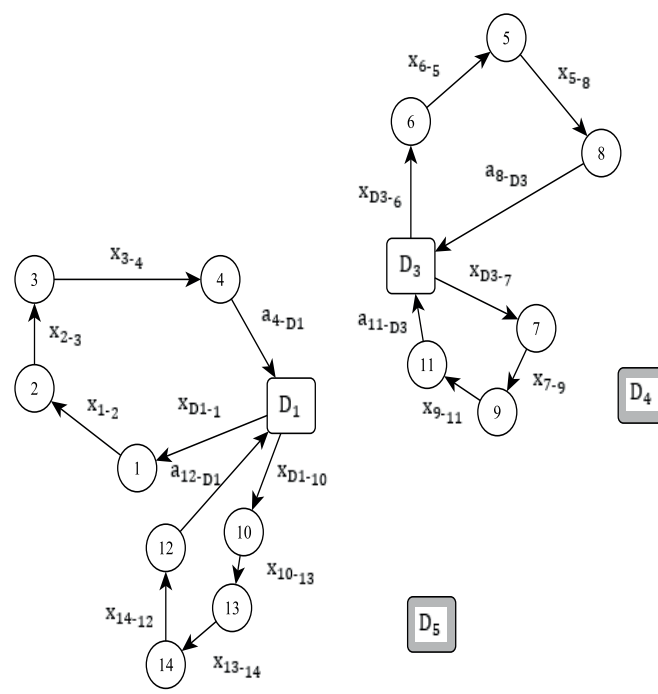

FigURA NRO. 17. CLRP estándar.

Elaboración propia.
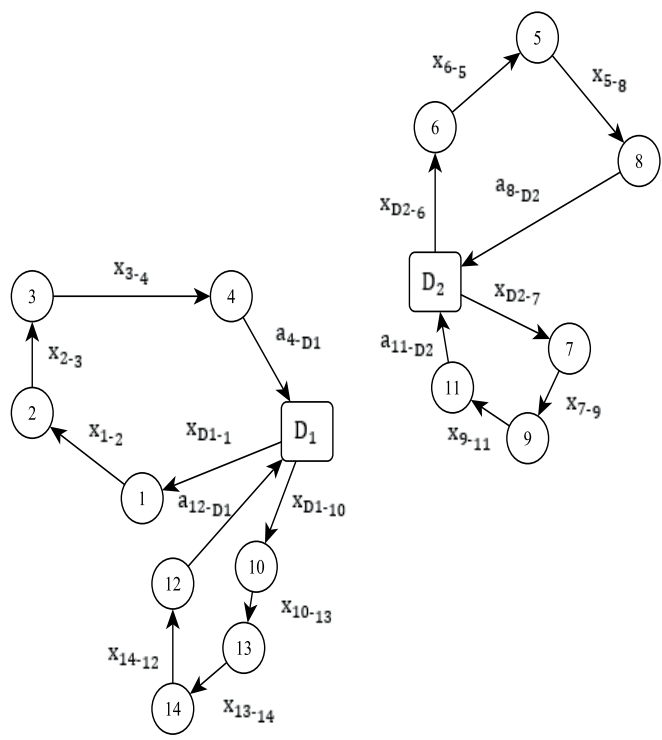

FigURA NRO. 18. MDVRP estándar.

Elaboración propia. 


\subsubsection{Problema de enrutamiento estándar (CVRP)}

Este modelo es conocido en la literatura especializada como «el problema de enrutamiento considerando restricciones de capacidad». Este problema establece un depósito de carga con capacidad ilimitada de productos a ser ofertados, desde donde se atiende un conjunto de nodos con demandas conocidas, como puede ser observado en la Figura nro.19. El tamaño de la flota de vehículos es limitado, así como la capacidad con la cual se sirven las rutas. El objetivo es confeccionar un conjunto de rutas, respetando las restricciones de capacidad de los vehículos y el número disponible.

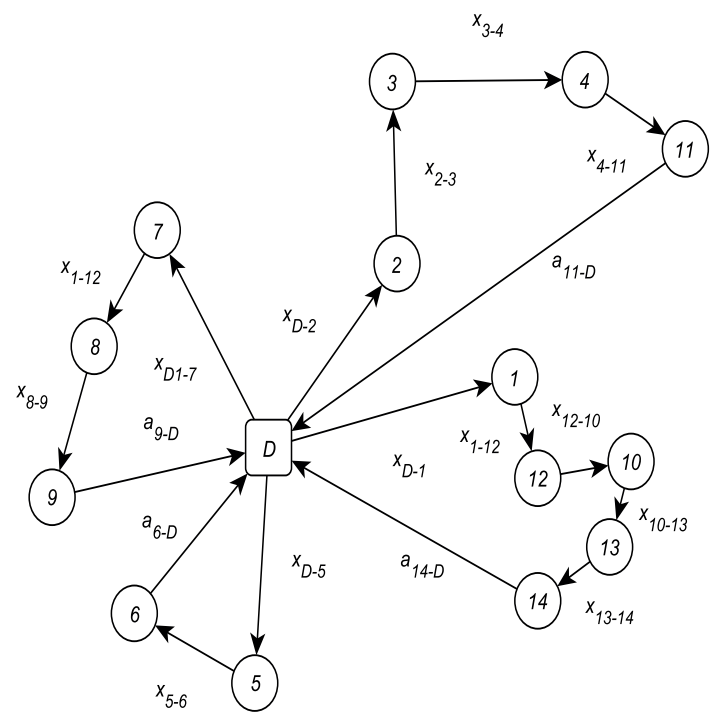

FigURA NRO. 19. CVRP.

Elaboración propia.

Como parámetro de entrada, este problema fija el número de depósitos a un valor de 1. Las trayectorias de las rutas son cerradas, inician y terminan en el mismo depósito de carga. El grafo empleado es identificado con las variables $x_{i j}$ y la variable $a_{i j}$. Este último corresponde al arco que conecta al último cliente servido con el depósito del cual parte la ruta. 
Eliana Mirledy Toro Ocampo, John Fredy Castañeda Londoño, Ramón Alfonso Gallego Rendón

$$
\min z=\sum_{i, j \in V} c_{i j} x_{i j}+\sum_{\substack{i=1 \\ j \in J}} c_{i j} a_{i j}
$$

La anterior ecuación 3.51 se encuentra sujeta a las ecuaciones desde la 3.13 a 3.39, y 3.48, 3.49. 


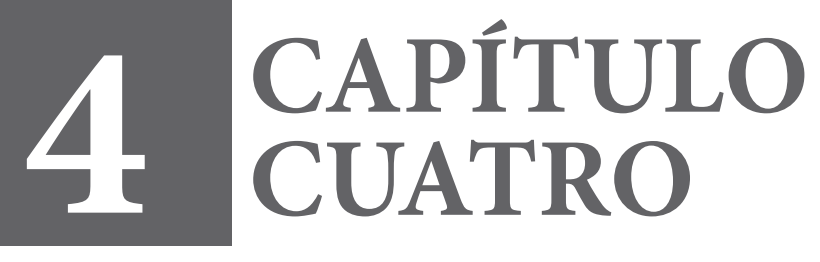




\section{PLANTEAMIENTO DEL MODELO MATEMÁTICO DEL GCLRP Y GOLRPC}

\subsection{Cálculo del consumo de energía del vehículo}

En este capítulo se describe el modelo matemático usado en el cálculo del consumo de energía entre dos nodos. El modelo es desarrollado con base en el equilibrio de fuerzas que actúan sobre el vehículo (ver Figura nro. 20).

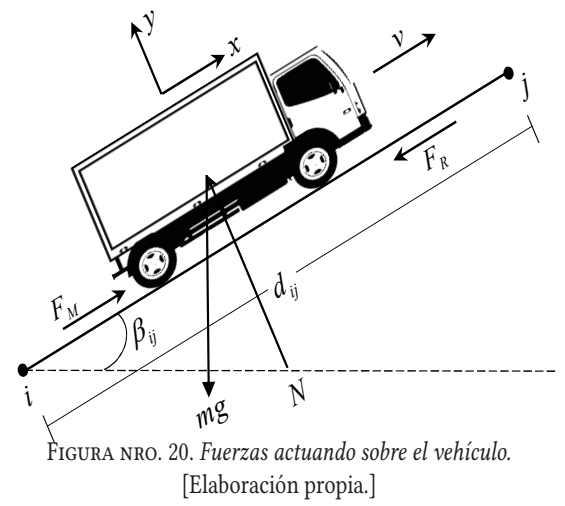

En la Figura nro. 20, el ángulo $\beta_{i j}$ representa el promedio de inclinación de la ruta entre los nodos $i$ y $j$, $\vec{F}_{R}$ representa las fuerzas que están opuestas al movimiento del vehículo en eje de $x \vec{F}_{M}$ representa las fuerzas son generadas por el motor del vehículo, transmitidas a las llantas; $m \vec{g}$, el peso del vehículo que incluye el vehículo y su 
Introducción al problema de enrutamiento de vehículos en la logística de distribución

carga (masa $m \times \vec{g}) ; \vec{N}$, la fuerza normal de la superficie inclinada sobre el vehículo; $v_{i j}$, la velocidad del vehículo en estado estacionario y $d_{i j}$, la distancia recorrida por el vehículo entre los nodos $i$ y $j$.

Las fuerzas $\vec{F}_{R}$, opuestas al movimiento del vehículo, se relacionan en la siguiente ecuación 4.1:

$$
\vec{F}_{R}=\vec{F}_{R, \text { llantas }}+\vec{F}_{R \text {,viento }}+\vec{F}_{R, \text { internas }}+\frac{m v_{i j}^{2}}{2 d_{i j}}
$$

El vector $\vec{F}_{R, \text { llantas }}$ es la fuerza ejercida entre las llantas sin tracción y el terreno, el cual se opone al movimiento del vehículo; $\vec{F}_{R \text {,viento }}$ representa la fuerza ejercida por el viento en contra del movimiento del vehículo; $\vec{F}_{R \text {,internas }}$ es la fuerza equivalente que se asocia a las fuerzas internas, opuestas al movimiento del vehículo; y $m v_{i j}^{2} / 2 d_{i j}$ representa la fuerza que se requiere para que el vehículo pueda alcanzar la energía cinética en estado estacionario. La masa del vehículo está compuesta por la masa del vehículo en vacío $m_{0}$ y la carga que es transportada entre los nodos $i$ y $j, t_{i j}$. Esta relacion se describe en 4.2:

$$
m=m_{0}+t_{i j}
$$

Para el desarrollo del equilibrio de fuerzas (con velocidad constante), se tienen las ecuaciones 4.3, 4.4, 4.5 y 4.6, así:

$$
\begin{array}{ll}
\sum F_{x}=m a_{x} & a_{x}=0 \\
\sum F_{y}=m a_{y} & a_{y}=0 \\
\sum F_{x}=m a_{x} \Rightarrow F_{M}-F_{R}-m g \sin \beta_{i j}=0 & \\
\sum F_{y}=m a_{y} \Rightarrow N-m g \cos \beta_{i j}=0 &
\end{array}
$$

Por definición, se sabe que $F_{R, \text { llantas }}=N b$, en la cual $b$ es una constante que depende de las características del terreno y del tipo de llanta del vehículo. Sustituyendo 4.1, 4.6 en 4.5, se obtiene 4.7:

$$
F_{M}=\left(m g \cos \beta_{i j}\right) b+F_{R, \text { viento }}+F_{R, \text { intermas }}+\frac{m v_{i j}^{2}}{2 d_{i j}}+m g \sin \beta_{i j}
$$

La fuerza transmitida por el motor a la llanta $F_{M}$ es multiplicada por la distancia $d_{i j}$, lo anterior equivale al trabajo y es expresado como, $U_{i j}=F_{M} d_{i j}$, Específicamente, puede verse en la ecuación 4.8, a saber: 


$$
U_{i j}=\left[\left(m_{0}+t_{i j}\right) g b \cos \beta_{i j}+F_{R, \text { viento }}+F_{R, \text { internas }}+\frac{\left(m_{0}+t_{i j}\right) v_{i j}^{2}}{2 d_{i j}}+\left(m_{0}+t_{i j}\right) g \sin \beta_{i j}\right] d_{i j}
$$

La energía requerida por el vehículo para desplazarse en el arco $i$ a $j$ está constituida por dos componentes: la primera es la requerida para desplazar la masa del vehículo operando en vacío y la segunda es la requerida para desplazar la carga, como se describe en 4.9 .

$$
\begin{aligned}
U_{i j}=\left[m_{0} g\left(b \cos \beta_{i j}+\sin \beta_{i j}+\frac{v_{i j}^{2}}{2 g d_{i j}}\right)+F_{R, \text { viento }}+F_{R, \text { internas }}\right] d_{i j} \\
+\left[g\left(b \cos \beta_{i j}+\sin \beta_{i j}+\frac{v_{i j}^{2}}{2 g d_{i j}}\right)\right] t_{i j} d_{i j}
\end{aligned}
$$

La energía para desplazar la masa del vehículo en vacío es calculada como se presenta en la ecuación 4.10:

$$
U_{i j}^{1}=\left[m_{0} g\left(b \cos \beta_{i j}+\sin \beta_{i j}+\frac{v_{i j}^{2}}{2 g d_{i j}}\right)+F_{R, \text { viento }}+F_{R, \text { internas }}\right] d_{i j}
$$

Esta expresión se simplifica al considerar que se asumen algunos valores constantes, como se describe en 4.11:

$$
\alpha_{i j}=m_{0} g\left(b \cos \beta_{i j}+\operatorname{sen} \beta_{i j}+\frac{v^{2}}{2 g d}+F_{R}(v)\right)
$$

$U_{i j}^{1}$ corresponde a la energía requerida para desplazar el vehículo en vacío en el $\operatorname{arco}(i, j): U_{i j}^{1}=\alpha_{i j} \times d_{i j}$ $\alpha_{i j}$ asume un valor constante que depende del peso del vehículo, de la inclinación del arco $(i, j)$, de la oposición al movimiento entre las ruedas del vehículo y el terreno en el que este se desplaza, de las características del camino, de las pérdidas internas en el vehículo, de la oposición del viento con respecto a la marcha de este y de la energía que requiere el vehículo para alcanzar la velocidad de estado estacionario.

La energía para desplazar la carga puede calcularse mediante en la ecuación 4.12:

$$
U_{i j}^{2}=\left[g\left(b \cos \beta_{i j}+\operatorname{sen} \beta_{i j}+\frac{v_{i j}^{2}}{2 g d_{i j}}\right)\right] t_{i j} d_{i j}
$$

Como en el caso anterior, la expresión es simplificada al asumir que algunos valores son constantes como se 
Introducción al problema de enrutamiento de vehículos en la logística de distribución

observa en en la ecuación (4.13):

$$
\gamma_{i j}=g\left(b \cos \beta_{i j}+\operatorname{sen} \beta_{i j}+\frac{v_{i j}^{2}}{2 g d_{i j}}\right)
$$

$U_{i j}^{2}$ corresponde a la energía que permite desplazar la carga en el $\operatorname{arco}(i, j): U_{i j}^{2}=\gamma_{i j} \times t_{i j} \times d_{i j}$

$\gamma_{i j}$ asume un valor constante, el cual depende de la inclinación del arco $(i, j)$, de la oposición al movimiento entre las llantas del vehículo y el terreno en el que se desplaza. La energía total que es requerida para desplazar el vehículo en el arco $(i, j)$ es descrita en 4.14 como:

$$
U_{i j}=U_{i j}^{1}+U_{i j}^{2}=\alpha_{i j} d_{i j}+\gamma_{i j} t_{i j} d_{i j}
$$

La energía requerida para desplazar el vehículo del nodo $i$ hacia el $j$ está compuesta de dos parte: la primera está relacionada con el vehículo en vacío y escrita como $\alpha_{i j} d_{i j}$, la segunda está relacionada con la carga y escrita como $\gamma_{i j} t_{i j} d_{i j}$. Si se asume una pendiente constante $\beta_{i j}$ para todos los arcos, la misma velocidad en todos los arcos, $\mathrm{y} \alpha_{i j}=\alpha \mathrm{y} \gamma_{i j}=\gamma$, se llega entonces a la siguiente expresión 4.15:

$$
U_{i j}=\alpha d_{i j}+\gamma t_{i j} d_{i j}
$$

La energía que requiere el vehículo para efectuar una ruta completa es calculada por las sumatorias de las energías requeridas por cada uno de los arcos. Al asociar el cálculo de la energía con las variables binarias $a_{i j}$ y $x_{i j}$, por el uso del arco $(i, j)$, se obtiene la siguiente expresión 4.16:

$$
\sum_{i, j \in V} U_{i j}=\alpha\left(\sum_{i, j \in V} d_{i j} x_{i j}+\sum_{i, j \in V} d_{i j} a_{i j}\right)+\gamma\left(\sum_{i, j \in V} d_{i j} t_{i j}\right)
$$

La cantidad de combustible que requiere la energía total $\sum_{i, j \in V} U_{i j}$ se obtiene usando un factor de conversión $E_{1}$ (galones/J). La cantidad de emisiones de partículas por unidad de combustible está haciendo uso de un segundo factor de conversión $E_{2}$ (kg por $\mathrm{CO}_{2}$ /galones). Por lo anterior, el valor total de emisiones es calculado en 4.17:

$$
E_{1} \times E_{2} \times \sum_{i, j \in V} U_{i j}=E \times \sum_{i, j \in V} U_{i j}
$$

El modelo anterior que fue desarrollado para el cálculo de las emisiones es del tipo lineal, tal como se muestra en 4.15. Es de aclarar que el modelo desarrollado anteriormente, es uno de los objetivos en la formulación del problema denominado «verde». 


\subsection{Análisis de costos asociados a la función objetivo}

Muchas de las compañías generalmente usan el servicio de arrendamiento leasing. Este arrendamiento, por lo general, corresponde a un valor mensual el cual se paga sobre activos fijos como pueden ser vehículos, aviones, maquinaria de producción, equipos industriales, edificaciones, etc. De esta manera, los costos pueden ser calculados usando el Valor Actual Neto (VAN) que percibe el arrendatario por el activo, como es presentado en la función 4.18. Al respecto, se puede visitar la propuesta de Trigeorgis y Mason (1987).

En las ecuaciones 4.18, 4.19, 4.20, $V_{0}$ corresponde al valor presente (costo) del activo arrendado; $L_{t}$ hace referencia al pago de arrendamiento del activo $t ; D_{t}$ representa el gasto de amortización en el tiempo $t$; $T$, la tasa de impuesto corporativo efectivo del arrendatario; $r_{B}$, corresponde al costo antes de impuestos de los préstamos; y $N$ es la vida útil del activo, es decir, el tiempo de vencimiento del contrato de arrendamiento.

Dado lo anterior, $O_{i}^{V A N}$ representa el VAN del costo de arrendamiento (leasing), asociado al empleo del depósito $i \in I$. $F^{V A N}$ corresponde al VAN, asociado al costo de arrendamiento (leasing), el cual está vinculado al uso del vehículo, asumiendo $F_{k}=F, \forall k \in K$.

$$
V A N=V_{0}-\sum_{t=0}^{N} \frac{I_{t}}{(1+r)^{t}} \equiv V_{0}-I
$$

Con

$$
\begin{gathered}
I_{t}=L_{t}(1-T)+D_{t} T \\
r=r_{B}(1-T)
\end{gathered}
$$

\subsection{Modelamiento matemático del problema GCLRP}

Con el fin de plantear un modelo matemático multiobjetivo para el problema de transporte de carga, se deben redefinir algunos parámetros y establecer algunos nuevos que están asociados al consumo de combustible. El problema del CLRP ha sido criticado debido a que su función objetivo mezcla la localización de los depósitos de carga, correspondiente a una decisión que se ubica en el nivel estratégico, con el costo de las rutas, que se considera como una decisión a nivel operativo. El problema de localización de los depósitos de carga requiere de la construcción de edificaciones y su dimensión está en función de la cantidad a almacenar. El costo que se asocia al transporte está definido en función del tamaño de la carga a ser servida. En los dos casos, depósitos de carga y las rutas, aparecen costos que se relacionan con la cantidad a producir, los costos de almacenamiento de inventarios y el tamaño de los 
Introducción al problema de enrutamiento de vehículos en la logística de distribución

envíos a los clientes finales. Por esta razón, se justifica la propuesta de proponer un modelo que lleva, hacia el VAN, a los costos que están asociados a la apertura de los depósitos y los asociados al uso de los vehículos.

\section{Párametros}

$O_{i}^{N P V} \quad$ Valor presente neto del costo de apertura del centro de distribución de carga.

$F^{N P V} \quad$ Valor presente neto que está asociado al costo del uso del vehículo.

$d_{i j} \quad$ Distancia recorrida entre los nodos $i$ y $j$.

$\alpha_{i j} \quad$ Parámetro que representa la cantidad de energía consumida por unidad de distancia $(\mathrm{J} / \mathrm{km})$, necesaria para que el vehículo en vacío se desplace entre los nodos $i$ y $j$.

$\gamma_{i j} \quad$ Parámetro que representa la energía adicional por unidad de distancia y toneladas de carga (J/km-ton) requerida por vehículo entre los nodos $i$ y $j$.

$E \quad$ Total de las emisiones por unidad de energía $\left(\mathrm{kg} \mathrm{de} \mathrm{CO}_{2} / \mathrm{J}\right)$.

TABLA NRo. 4. Parámetros del modelamiento matemático del problema GCLRP. Elaboración propia.

$$
\min z=\Psi_{1}, \Psi_{2}
$$

Conforme a lo anterior, la ecuación 4.24 se encuentra sujeta a las ecuaciones desde 3.13 a 3.39, 3.48 y 3.49 .

El modelo matemático del G-CLRP es propuesto usando funciones objetivo de costo y de impacto ambiental las cuales deben ser minimizadas. Estas son expresadas en 4.22 y 4.23 como:

$$
\Psi_{1}=\sum_{i \in I} O_{i}^{N P V} y_{i}+\sum_{i \in I, j \in J} F^{N P V} a_{i j}+\sum_{i, j \in V} c_{i j} x_{i j}+\sum_{i \in I, j \in J} c_{i j} a_{i j}
$$

y

$$
\Psi_{2}=\alpha E\left(\sum_{i, j \in V} d_{i j} x_{i j}+\sum_{i \in I, j \in J} d_{i j} a_{i j}\right)+\gamma E \sum_{i, j \in V} d_{i j} t_{i j}
$$

En el primer objetivo, es representado el costo de la operación. En este, la primera sumatoria hace referencia a costos fijos, en los cuales se incurre por colocar en operación los depósitos; la segunda sumatoria corresponde a los costos fijos de utilización del vehículo asociado a la rutas. De esa índole, las sumatorias finales están relacionadas con los arcos activos que representan las rutas diseñadas. 
En el segundo objetivo se representan las emisiones ambientales, asociadas al consumo de combustible, el cual fue desarrollado en la sección anterior.

El modelo asume igual inclinación y velocidad constante en todos los arcos. Dadas las condiciones anteriores, $\alpha$ y $\gamma$ asumen valores constantes e iguales para todos los arcos. En el caso de considerar diferentes valores de inclinación y/o velocidad en los arcos, $\alpha_{i j}$ y $\gamma_{i j}$ deben ser calculados para cada tramo. Para los dos casos señalados anteriormente, el modelo de emisiones es de tipo lineal.

La expresión que representa el consumo de combustible está dividida en dos partes. La primera corresponde a la cantidad de energía requerida para desplazar el vehículo en vacío, donde $x_{i j}$ representan los arcos activos. Los $a_{i j}$ representan los arcos de retorno al depósito. La segunda corresponde a la cantidad de energía requerida para transportar la carga.

$\alpha$ es un parámetro que representa cantidad de energía por unidad de distancia y está dado en $\mathrm{J} / \mathrm{km}, \gamma$ es un parámetro que representa cantidad de energía por unidad de carga y de distancia, y está dado por $\mathrm{J} / \mathrm{km}$-ton. Para la conversión a emisiones de partículas, son multiplicados por el parámetro $E$.

En Toro et al. (2017b), se presenta el modelo matemático y el método de solución del problema GCLRP. Además, se lleva a cabo un análisis de resultados usando instancias de la literatura. En la solución, se usa un método de optimización exacto denominado Epsilon-Constraint.

\subsection{Formulación del problema GOLRP}

La formulación del modelo matemático del GOLRP está basado en el modelo matemático que fue planteado para el OLRP y sigue la siguiente estructura definida en la función objetivo 4.24:

$$
\min \Psi_{1}, \Psi_{2}
$$

Conforme a lo anterior, la ecuación 4.24 se encuentra sujeta a las ecuaciones desde 3.13 a 3.39, 3.44 y 3.45 .

Los resultados obtenidos, basados en esta propuesta, se han publicado en Toro et al. (2017a). Este trabajo es uno de los primeros reportados en esta temática, donde se estudia el problema de localización y enrutamiento con rutas abiertas en un ambiente biobjetivo. 


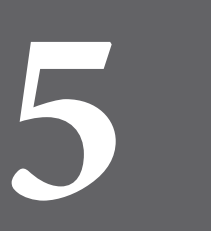

CAPÍTULO CINCO 


\section{METODOLOGÍA DE SOLUCIÓN APROXIMADA}

Los problemas de optimización pueden ser solucionados de forma aproximada mediante el uso de heurísticas constructivas, y metaheurísticas a través del desarrollo de algoritmos evolutivos y de trayectoria. Estas últimas son los más usadas debido a que, comúnmente, generan los mejores resultados. Adicionalmente, es posible realizar implementación a problemas multiobjetivo con mejor desempeño que las metodologías de modelos matemáticos exactos. Esta metodología permite su utilización en cualquier tipo de problema de ruteo. En esta implementación, es aplicada la metodología aproximada al problema de ruteo abierto y cerrado, asociado a flotas propias y subcontratadas, respectivamente.

La metodología de solución aproximada es aplicada como multiobjetivo al problema específico, denominado Green Vehicle Routing Problem with Private Fleet and Common Carriers with Equity Workload (GVRPPCWE) que, además de usar los dos tipos de flota, incluye en la función objetivo la cantidad de emisiones contaminantes y un criterio de equidad en las rutas. La metaheurística utilizada es denominada Iterated Local Search (ILS), la cual implementa la generación de subconjuntos en el espacio de soluciones alrededor de soluciones óptimas locales, en las cuales se ejecutarán procedimientos de búsqueda local aplicando adecuados esquemas de vecindad. Para salir de los óptimos locales, se aplican procedimientos de diversificación que permiten salir a otros espacios de solución. Para la búsqueda local, son aplicadas las características del Random Variable Neighborhood Search (RVNS) que se basa en la utilización aleatoria de operadores de búsqueda en los vecindarios denominados intra-ruta e inter-ruta. El algoritmo comprende los siguientes pasos: (1) generación de la solución inicial local, (2) búsqueda local, (3) perturbación, (4) dominancia y (5) criterio de aceptación.

Las soluciones encontradas por esta metodología son frentes o fronteras de Pareto, asociados a las tres dimensiones de las funciones objetivo (costo, reducción de emisiones de $\mathrm{CO}_{2}$ y equidad laboral o balance de rutas). Para evaluar la calidad de un frente, es necesario la aplicación de métricas geométricas que permitan clasificar los frentes por su calidad. Estas métricas son descritas en detalle en la sección 2.6.1.1. 


\subsection{Formulación multiobjetivo para el GVRPPCWE}

La construcción de vías para resolver los problemas de ruteo puede hallarse de la analogía del problema operativo de redes de distribución de energía (Lavorato et al., 2012). Estas redes son operadas de forma radial y reconfiguradas buscando minimizar las pérdidas. Las topologías formadas son grafos tipo árbol, es decir, los nodos pueden tener un grado mayor o igual a 2. Para los problemas de ruteo, el grado de los nodos será de 2. Por lo tanto, es posible proponer un modelo para resolver los problemas de ruteo con base en el planteado para redes de distribución, incluyendo restricciones que garanticen el cumplimiento del grado de los nodos. En este problema, se tienen las siguientes consideraciones:

1. Se cuenta con un único depósito, donde los vehículos propios retornarán posterior a visitar el último cliente y los vehículos subcontratados no deberán volver al depósito.

2. La capacidad del depósito es ilimitada o suficiente para atender la demanda.

3. Los vehículos tienen capacidad limitada y uniforme, incluyendo los vehículos subcontratados. Esto hace que el problema se denomine homogéneo.

4. El conjunto de clientes $i$ tienen una demanda conocida y la suma es mayor a la capacidad de los vehículos propios $k$.

5. La red asociada al problema consta de dos grafos completos; el primero, asociado a los arcos recorridos por las rutas propias; y el segundo, a las subcontratadas.

6. Cada cliente será visitado una única vez con cualquiera de los vehículos, ya sean propios o subcontratados.

7. Los vehículos de las rutas subcontratadas podrán realizar solo un recorrido y este termina su recorrido en el último cliente atendido.

Las emisiones de $\mathrm{CO}_{2}$ son obtenidas directamente del cálculo del consumo de combustible de los vehículos. El modelo utilizado en este trabajo es el implementado en Toro et al. (2017b), el cual está definido a través de la naturaleza de las fuerzas que intervienen en el movimiento de los vehículos. Para determinar el criterio de equidad, son definidos $l_{k}$ y $l_{u}$, como la distancia recorrida por los vehículos $k$ y $u$; y a $N$, como el conjunto de rutas donde $k, u \in N$. El valor cuantificable de equidad se obtiene de la diferencia entre la mayor y menor distancia de todas las rutas. A este criterio, se le denomina rango y se describe en la ecuación 5.1 como:

$$
R=\max _{k \in N} l_{k}-\min _{u \in N} l_{u}
$$


El GVRPPCWE se obtiene con base en la formulación propuesta por Lavorato et al. (2012) para problemas de flujo de carga en redes de distribución de energía, y se define como un problema lineal entero mixto con los siguientes conjuntos y ecuaciones: el problema es representado como un grafo completo de la forma $G=(V, A)$, donde $V$ representa un conjunto con la totalidad de los vértices del grafo (depósitos y clientes), y el conjunto de arcos que conectan los clientes y el depósito. Se tiene el conjunto $J=\{1,2,3, \ldots n\}$, que contiene los nodos de clientes (el depósito tendrá siempre asignado el nodo cero). En la Tabla nro. 5, se presentan las variables de la presente ecuación, a saber:

\section{Conjuntos:}

$J$ : $\quad$ Conjunto de clientes.

I: $\quad$ Conjunto de depósitos (en este caso, es único).

$V$ : Conjunto de nodos, $V=I \cup J$.

\section{Variables binarias de decisión:}

$c_{i j}$ : Costo asociado al recorrido entre los nodos $i-j$.

$x_{i j}$ : Variable que representa la activación de los arcos recorridos por la flota propia.

$a_{i j}$ : Variable que representa el arco de retorno al depósito.

$s_{i j}$ : Variable que representa la activación de los arcos recorridos por la flota subcontratada.

\section{Parámetros:}

$d_{i j}: \quad$ Distancia entre los clientes $i$ y $j$.

$\alpha$ : Constante en función a la inclinación, peso vehículo vacío, energía para velocidad transitoria, resistencia de ruedas y viento, y las perdidas internas del vehículo.

$\gamma$ : $\quad$ Constante en función de la inclinación del terreno y la resistencia de las ruedas.

E: $\quad$ Emisiones por unidad de combustible.

$R$ : $\quad$ Criterio de equidad.

$P: \quad$ Penalización por la utilización de la flota subcontratada.

$t_{i j}$ : $\quad$ Flujo de mercancías entre los nodos $i-j$ para la flota propia.

TABLA NRO. 5. Definición de variables para el GVRPPCWE. Elaboración propia.

Las funciones para el costo, el impacto ambiental y la equidad se definen en las ecuaciones 5.2, 5.3 y 5.4 como:

$$
\Psi_{1}=\sum_{i, j \in V} c_{i j} x_{i j}+\sum_{j \in V} c_{j-0} a_{j-0}+P \sum_{i, j \in V} c_{i j} s_{i j}
$$




$$
\begin{gathered}
\Psi_{2}=\alpha E\left(\sum_{i, j \in V} d_{i j} x_{i j}+\sum_{i, j \in V} d_{i j} a_{i j}\right)+\gamma E\left(\sum_{i, j \in V} d_{i j} t_{i j}\right) \\
\Psi_{3}=R
\end{gathered}
$$

La descripción de las ecuaciones que componen las restricciones se encuentra de forma detallada en Castaneda et al. (2020). La ecuación 5.2 describe la función objetivo como la suma de los costos. La ecuación 5.3 relaciona las emisiones con el consumo de combustible de los vehículos que, a su vez, es obtenido de la distancia recorrida y la carga como: $\alpha=0.1581 / 1000 / 2, \gamma=0.001581 / Q * 20 / 2$ y $E=8.70645 \mathrm{~kg}$ de $\mathrm{CO}_{2}$ por galón. La ecuación 5.4 muestra que la tercera función objetivo está dada como la equidad definida por la ecuación 5.1 .

\subsection{Algoritmo Iterated Local Search (ILS)}

La metaheurística ILS implementa la generación de subconjuntos en el espacio de solución, alrededor de soluciones óptimas locales en las cuales se ejecutan procedimientos de búsqueda local, aplicando adecuados esquemas de vecindad. Para salir de los óptimos locales, se aplican procedimientos de diversificación que permiten salir a otros espacios de solución. Para la búsqueda local, se define el Random Variable Neighborhood Search (RVNS) como la selección aleatoria de los operadores en la búsqueda local, denominados intra-ruta e inter-ruta. Las características del RVNS se basan en la utilización de los operadores en los vecindarios variables para realizar la búsqueda local.

La implementación del algoritmo ILS comprende los siguientes pasos:

1. Generación de la solución inicial local: Se construye una solución usando una heurística constructiva y, a partir de esta, se inicia la búsqueda local que permite llegar a mejores soluciones. En este trabajo, se usó el algoritmo de ahorros modificado que es la solución de partida del algoritmo. La construcción de la solución inicial varía a través de la ejecución general, permitiendo elegir aleatoriamente entre la solución única del algoritmo de ahorros y las alternaciones a esta. Lo anterior permite obtener múltiples puntos de partida para el algoritmo ILS.

2. Búsqueda Local: Aplica mejoramientos continuos a través de la búsqueda dentro de los vecindarios. Estas búsquedas se realizan por medio de operadores que aplican movimientos inicialmente inter-ruta y posteriormente -a la mejor solución encontrada- se realizan movimientos intra-ruta.

3. Perturbación: Aplicación de movimientos aleatorios inter-ruta (sin importar si existe mejoramiento). A partir de estos movimientos, se logra escapar de óptimos locales y llegar a otras soluciones del espacio de solución 
del problema. Estos movimientos también son denominados de diversificación, ya que permiten al algoritmo encontrar un nuevo punto desde donde se inicia un proceso de búsqueda local.

4. Criterio de dominancia: Selecciona las mejores soluciones y construye con estas el frente de Pareto.

5. Criterio de aceptación: Determina cuando el algoritmo debe detenerse en la búsqueda de soluciones. Este criterio básicamente es la definición de un número máximo de iteraciones en la búsqueda local y en la ejecución del algoritmo completo, es decir, se tendrán dos parámetros como criterio de aceptación que se definen como:

- Iteraciones locales del ILS: corresponde al número de iteraciones del algoritmo donde se ejecutan los pasos del 1 al 3 .

- Iteraciones globales del ILS: corresponde al número de iteraciones donde se inicia nuevamente el algoritmo completo y finaliza con la obtención de una nueva solución del espacio de búsqueda.

A diferencia de los algoritmos evolutivos, el ILS no se detiene a través de un parámetro de tolerancia obtenido de las variaciones en las soluciones encontradas. Debido a la capacidad de diversificación del algoritmo, un parámetro de tolerancia no sería conveniente, ya que el algoritmo podrá incluso encontrar la misma solución repetidas veces a través de diferentes vecindarios, pero tendrá la posibilidad de continuar con la búsqueda durante el número de iteraciones que haya definido.

El algoritmo general del ILS multiobjetivo es presentado en el algoritmo 1. Las variables son descritas en la Tabla nro. 6

\begin{tabular}{lll}
\hline Variable & Tipo & Descripción \\
\hline DF & Entero & Factor de profundidad de la búsqueda local. \\
MaxIter & Entero & Cantidad de iteraciones globales del algoritmo. \\
MaxIterLocal & Entero & Cantidad de iteraciones locales del algoritmo. \\
$n$ & Entero & Número de clientes o nodos. \\
B & Entero & Relación entre la cantidad de iteraciones locales y el número de vehículos del \\
& & problema. \\
Entero & Número de vehículos. \\
SS & Arreglo & Solución inicial del algoritmo de ahorros. Tiene dimensiones de $[V][q]$. \\
& & $V$ : número de vehículos.
\end{tabular}


Introducción al problema de enrutamiento de vehículos en la logística de distribución

$\begin{array}{lll}\text { Pareto } & \text { Arreglo } & \text { Arreglo multidimensional }[V][q][N] \text { que almacena las soluciones encontradas no } \\ & & \text { dominadas. } \\ & N \text { : límite de almacenamiento de soluciones no dominadas. } \\ \text { So } & \text { Arreglo } & \text { Contiene la solución inicial general del algoritmo ILS. } \\ \text { S1 } & \text { Arreglo } & \text { Contiene la mejor solución actual local. } \\ \text { F, G,D } & \text { Entero } & \text { Funciones objetivo para el costo, emisiones de } \mathrm{CO}_{2} \text { y valor de equidad, } \\ & & \text { respectivamente. }\end{array}$

TABLA NRO. 6. Variables generales ILS.

Elaboración propia.

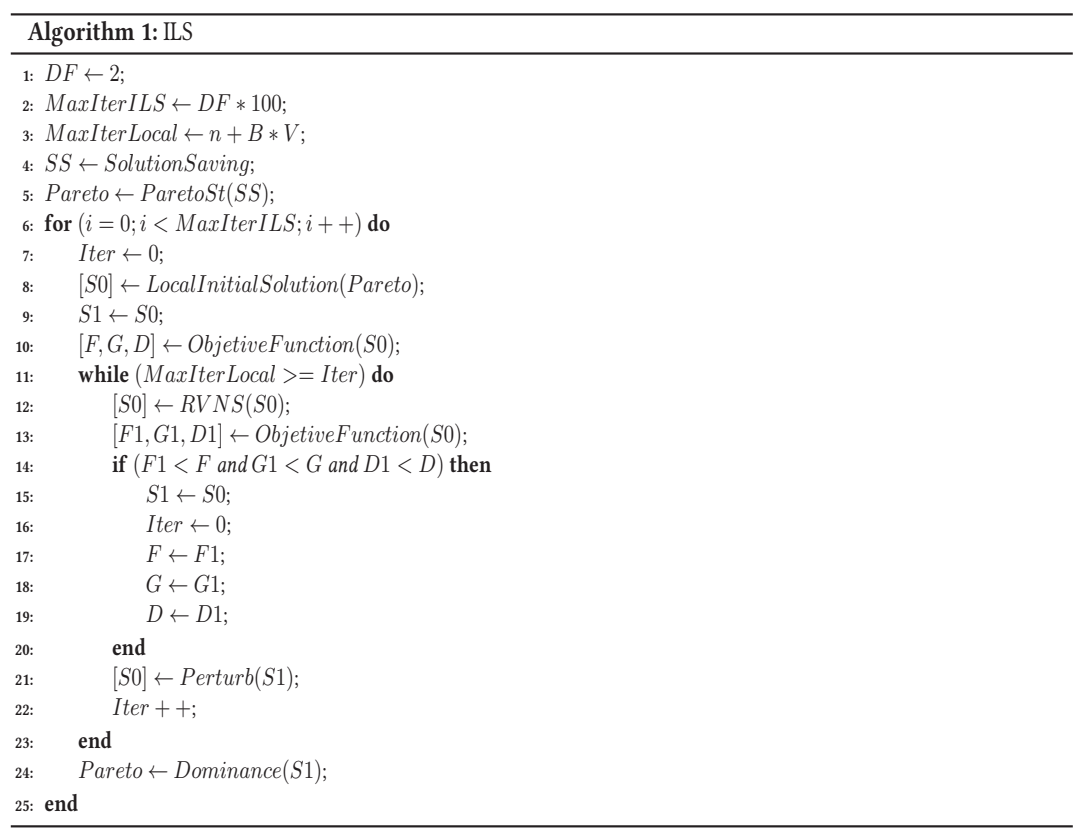




\subsection{Descripción del algoritmo ILS multiobjetivo}

Luego de obtener la solución del algoritmo de ahorros en la línea 4, es generado el Pareto a través de la función ParetoST, la cual genera un arreglo multidimensional que contiene en todos sus índices la solución del algoritmo de ahorros (línea 5). A partir de la línea 6, se inician las iteraciones globales del algoritmo ILS. En la línea 8, es generada la solución inicial para la búsqueda local. La función LocalInitialSolution selecciona una solución del Pareto, que fue llenada con la solución del algoritmo de ahorros. En la línea 11, se inicia la búsqueda local. En la línea 12, el RVNS utiliza la solución inicial obtenida en la línea 8 para realizar la búsqueda local a través de la aplicación de operadores inter-ruta e intra-ruta. En la línea 14, se verifica si esta solución mejora de forma simultánea las tres funciones objetivo. Esto permite que el algoritmo trabaje sobre cada solución disponible. En caso de que haya mejoramiento, son actualizadas las variables para una nueva iteración local. Posteriormente, para iniciar nuevamente la búsqueda local, es aplicado un procedimiento de diversificación a través de la función de perturbación en la línea 21. Esta solución será nuevamente ingresada al RVNS en una nueva iteración local. Finalmente, en la línea 24, es aplicada la función de dominancia, la cual verifica que la solución obtenida no sea dominada y la incluye de forma ordenada en el Pareto, remplazando las soluciones del algoritmo de ahorros que ocupan la variable. La descripción del procedimiento de dominancia se realiza en la sección 2.6.1.

\subsection{Solución inicial local del ILS}

La solución inicial local del ILS consiste en seleccionar una de las soluciones disponibles del Pareto actual. Para esta solución, se define una probabilidad para que sea seleccionada del Pareto y se le aplique un procedimiento leve de perturbación. Este permitiría contar con un adecuado rango de diversificación en el punto de inicio de cada iteración. Las soluciones seleccionadas que no sean perturbadas ingresarán directamente al RVNS, tal como se encuentran en el Pareto. El procedimiento de perturbación será descrito en la sección 5.9.

Este procedimiento hace que cada solución del Pareto sea una solución de inicio que será susceptible a ser mejorada por la búsqueda local. Como se mencionó en la descripción del algoritmo ILS, la solución obtenida por el algoritmo de ahorros será la más usada en las primeras iteraciones. A medida que avanza la ejecución, estas soluciones se irán reemplazando por nuevas encontradas. En el algoritmo 2, se muestra el pseudocódigo para la selección de la solución inicial local del ILS. En la Tabla nro. 7, se muestra la definición de las variables utilizadas en el algoritmo 2.

\begin{tabular}{lll}
\hline Variable & Tipo & Descripción \\
\hline$P F$ & Entero & Factor de perturbación aplicado a los operadores.
\end{tabular}




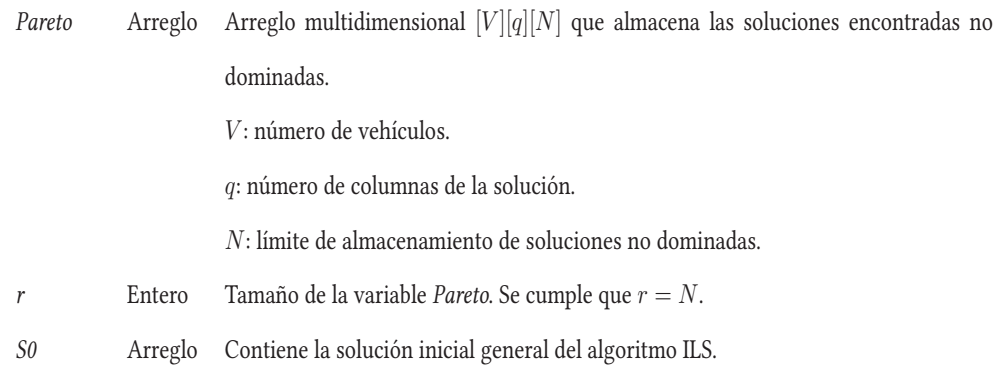

TABLA NRO. 7. Variables solución inicial local ILS. Elaboración propia.

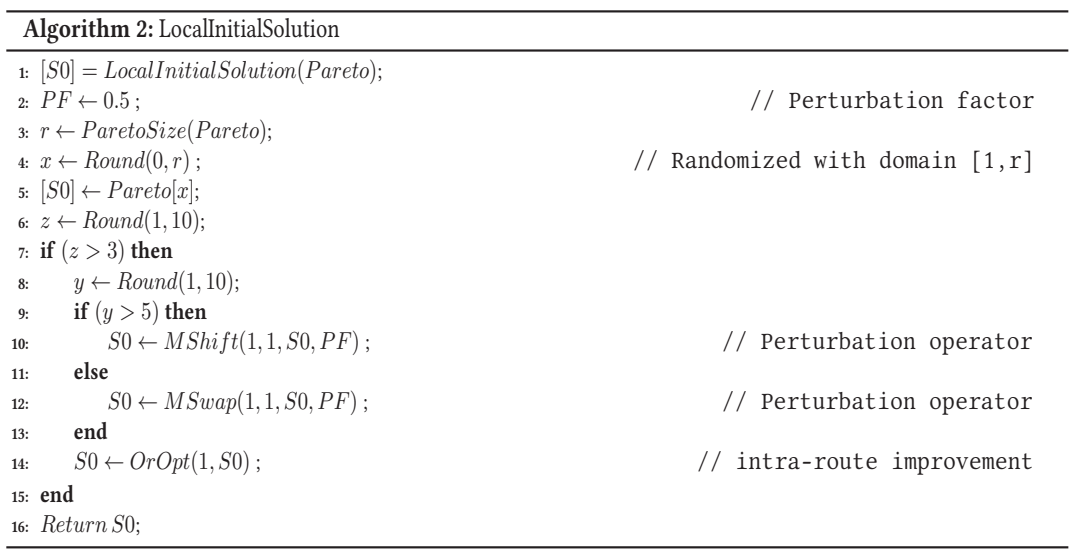

\subsubsection{Descripción del código de la solución inicial local del ILS}

La solución inicial es definida por medio de la función de la línea 1. En la línea siguiente, es definido un factor de perturbación que permite modularla. La función ParetoSize indica el número de soluciones que contiene el Pareto; de las cuales, en la línea 5, una será seleccionada aleatoriamente. El condicional de la línea 7 define la aplicación de perturbación a la solución inicial, seleccionada del Pareto. En esta implementación, se define con una probabilidad de ser perturbada del $70 \%$ en cada iteración. Entre las líneas 9 y 13 se aplica la perturbación a la solución inicial; y finalmente, en la línea 14, se realiza un mejoramiento intra-ruta. 


\begin{tabular}{lll}
\hline Variable & Tipo & Descripción \\
\hline S0 & Arreglo & $\begin{array}{l}\text { Solución inicial del ILS. Tiene dimensiones de }[V][q] . \\
V: \text { número de vehículos. } \\
\text { q: número de columnas del arreglo que contiene la solución. }\end{array}$ \\
F1, G1, D1 & Entero & $\begin{array}{l}\text { Función objetivo inicial para el costo, emisiones de } \mathrm{CO}_{2} \text { y valor de equidad, } \\
\text { respectivamente. }\end{array}$ \\
F2, G2, D2 & Entero & Funciones objetivo posteriores a intercambios inter-ruta. \\
NLInter & Arreglo & Conjunto que contiene los operadores inter-ruta. \\
NLIntra & Arreglo & Conjunto que contiene los operadores intra-ruta. \\
SS & Arreglo & Solución obtenida posterior a la aplicación de mejoramiento intra-ruta. \\
Fs & Entero & Función objetivo del costo. \\
\hline
\end{tabular}

TABLA NRO. 8. Variables algoritmo RVNS.

Elaboración propia.

\subsection{Random Variable Neighborhood Search (RVNS)}

Esta función agrupa, básicamente, la búsqueda local con la característica de construcción de vecindarios elegidos aleatoriamente de un conjunto definido $N L=\left[N^{1}, N^{2}, N^{3} \ldots, N^{n}\right]$. Estos vecindarios son construidos a través de estructuras de intercambio inter-ruta, donde cada vecino corresponde a una solución factible, formada del intercambio de clientes entre rutas. El RVNS explora todos los vecinos de manera exhaustiva y almacena la mejor solución.

Se empieza con la solución inicial obtenida, como se muestra en la sección 5.4. Posteriormente, se elige un operador del conjunto $N L$ de manera aleatoria, el cual realiza una exploración de manera exhaustiva del vecindario por medio de la aplicación de operadores de intercambio inter-ruta. Estos son eliminados si no mejoran la solución. En el caso de mejora, se aplica una etapa de mejoramiento por medio de la selección de un operador intra-ruta. Los operadores intra-ruta también son eliminados cuando no mejoran la solución y terminan su proceso de búsqueda cuando no haya operadores disponibles. Posterior a los mejoramientos intra-ruta, se reinician todos los operadores. Cuando todos los operadores inter-ruta son eliminados, el proceso termina. El procedimiento es descrito en el algoritmo 3. En la Tabla nro. 8, se definen las variables utilizadas en al procedimiento. 


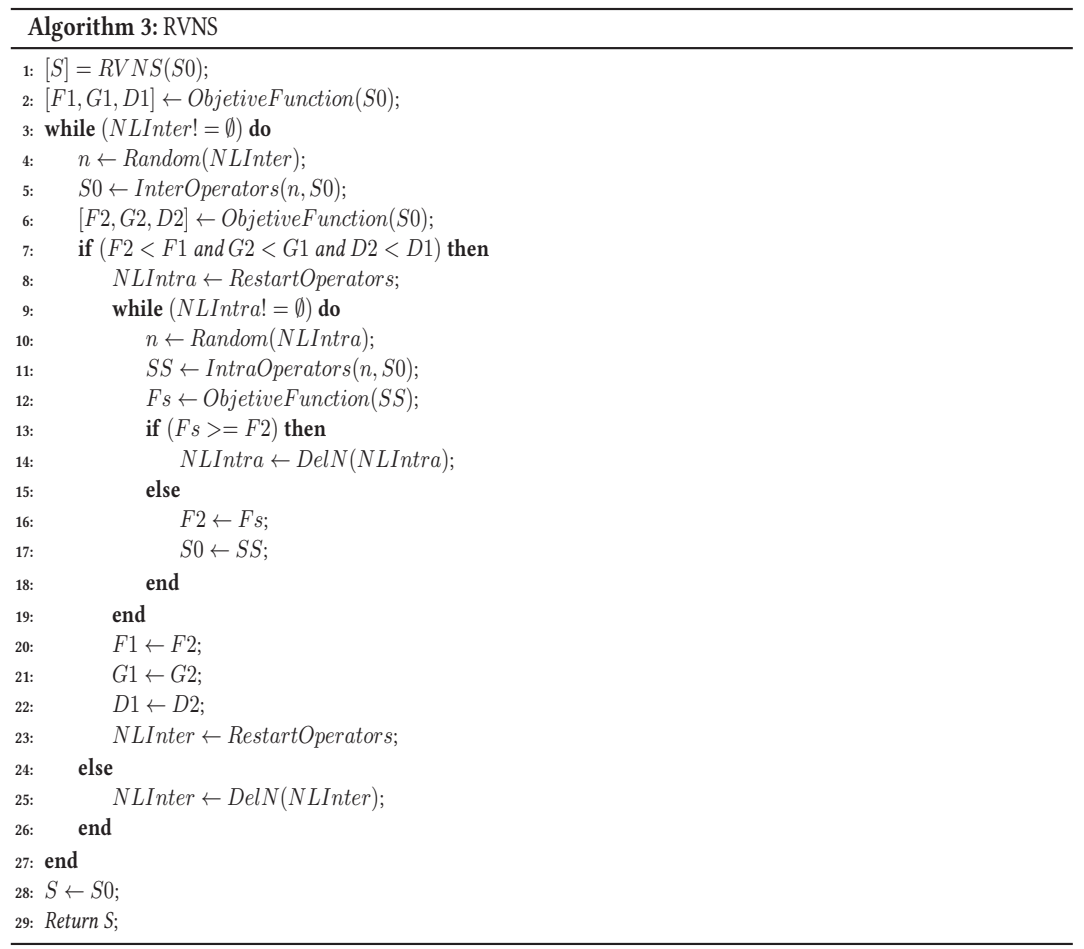

\subsubsection{Descripción del algoritmo para el RVNS}

En la línea 1, es definida la función RVNS, la cual tiene, como parámetro de entrada, una solución de inicio de búsqueda. La función ObjetiveFunction obtiene los valores de las funciones objetivo de la solución de inicio. A partir de la línea 3, comienza el algoritmo que tiene un criterio de terminación cuando ya no existan operadores inter-ruta disponibles. En la línea 4, es seleccionado aleatoriamente un operador inter-ruta, el cual es aplicado en la línea 5. En la línea 7, se encuentra el condicional de mejoramiento que indica que todas las funciones objetivo deben ser mejoradas por los operadores inter-ruta. Este condicional hace que cada solución del Pareto sea mejorada por otra que la domina estrictamente (se ofrecen más detalles en el subapartado 5.5.2). El concepto de dominancia se define en la sección 2.6.1. En la línea 9, inicia el mejoramiento intra-ruta que también es seleccionado de forma aleatoria. El condicional de la línea 13 solo verifica mejoramiento en la función objetivo del costo ya que, al ser movimientos de clientes en una misma ruta, las emisiones de $\mathrm{CO}_{2}$ disminuyen proporcionalmente y el criterio de equidad no varía significativamente. Los operadores que no mejoran la solución son eliminados en la línea 14. Luego del mejoramiento intra-ruta, los 
operadores inter-ruta son reiniciados para comenzar de nuevo la búsqueda sobre la mejor solución. En la línea 25 son eliminados los operadores intra-ruta que no mejoran la solución.

\subsubsection{Metodología de búsqueda del algoritmo ILS multiobjetivo}

El ILS puede considerarse como un algoritmo de búsqueda con un alto grado de tendencia descendente debido a que centra su búsqueda en la ubicación de una solución especifica. Además, a través de la construcción de estructuras de vecindad, puede explorar de manera intensa el espacio de soluciones vecino.

En la metodología de búsqueda para problemas con varios objetivos, el factor que indica si una solución es mejor que otra lo indica la dominancia, pero esta no podría ser utilizada en la búsqueda local debido a que, por la definición de dominancia (ver sección 2.6.1), la búsqueda podría no converger hacia las soluciones óptimas y atascarse en bucles de búsqueda. Por esta razón, el algoritmo es definido mediante la condición: una solución es mejor que otra en la búsqueda local, cuando es estrictamente mejor en todos los objetivos simultáneamente. Esto es realizado por la instrucción de la línea 7 del algoritmo 3. Lo anterior, implica que el algoritmo ILS realice una búsqueda local para cada solución con operadores inter-ruta. Estos obtendrán soluciones únicamente en su espacio o región de dominancia. De esta forma, el algoritmo mejora el frente de Pareto. En la Figura nro. 21 se muestra el espacio donde se ubican las soluciones que obtiene el algoritmo ILS en un problema con dos objetivos. Para tres o más objetivos, los vectores de descenso apuntarían al origen para problemas de minimización en todos sus objetivos.

\subsection{Movimiento tabú}

La implementación de un algoritmo como el ILS implica realizar gran cantidad de movimientos entre rutas y verificar el valor de las funciones objetivo. Por esto, es necesario evitar realizar movimientos y cómputos repetitivos que no mejoren las funciones objetivo. Por lo tanto, se toma como referencia la metaheurística «búsqueda tabú», la cual implementa una memoria de corto plazo haciendo que una parte de los movimientos sean prohibidos de forma temporal.

En este trabajo, se definen como movimientos tabú aquellos que ya han sido verificados mediante los operadores de intercambio intra-ruta y que no mejoran la función objetivo. Debido a que el ILS aplica un procedimiento de perturbación a algunas o todas las rutas, las modificadas se habilitarán nuevamente. Para implementar esto, se define el arreglo multidimensional 5.5 como:

$$
\operatorname{Mtabu}\left[r_{1}\right]\left[r_{2}\right][n]=0
$$


Donde $n \in N L$, y $r_{1}$ y $r_{2}$ corresponden a las rutas usadas para el intercambio inter-ruta. Los movimientos tabú son almacenados en $M t a b u$, donde $n$ es uno de los vecindarios pertenecientes a $N L=\left[N^{1}, N^{2}, N^{3} \ldots, N^{n}\right]$. Cada operador de intercambio inter-ruta estará definido en uno de estos vecindarios. Cuando el movimiento no mejora la función objetivo, el valor correspondiente a ese movimiento será 0 en el arreglo. Por ejemplo, el operador de intercambio $n=2$ realizó el movimiento entre las rutas $r_{1}=3$ y $r_{2}=6$ de una solución con seis vehículos; si el movimiento no mejora la función objetivo, el valor se actualiza como Mtabu[3][6][2] = 0; de lo contrario, el valor permanecerá en 1. Posteriormente, si en la búsqueda se intenta realizar el mismo movimiento, este será bloqueado. Cuando se aplica el procedimiento de perturbación sobre las rutas $r_{1}=3$ y $r_{2}=6$, se actualiza la variable como $\operatorname{Mtabu}[3][6][n]=1$ para todos los vecindarios $N L$.

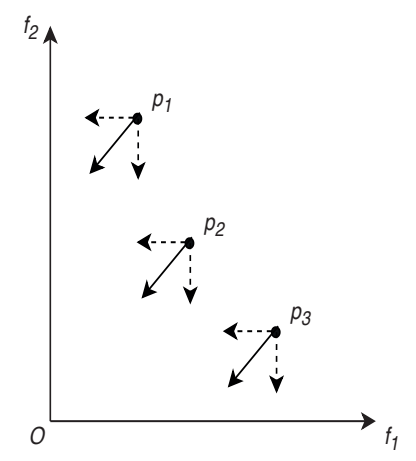

\section{Figura NRo. 21. Metodología búsqueda ILS.} Elaboración propia.

\subsection{Operadores de intercambio inter-ruta}

Los operadores intra-ruta son estructuras encargadas de crear vecindarios por medio de intercambio de clientes entre las rutas. La elección de cada estructura se realiza de manera aleatoria. Para cada iteración, se descartan las que no mejoran la solución. Se finaliza cuando todos hayan sido descartados. Las estructuras inter-ruta son descritas a continuación:

- Operador shift: transfiere un cliente desde una ruta $r_{1}$ a la ruta $r_{2}$. El nuevo cliente que llega a la ruta $r_{2}$ construirá una cantidad de vecindarios, equivalentes al número de posiciones posibles donde se pueda ubicar en la ruta $r_{1}$. Este intercambio se realiza de manera exhaustiva, es decir, todos los nodos serán probados en cada una de las rutas, en todas sus posiciones posibles. 
- Operador swap: realiza intercambios entre un par de rutas $r_{1}$ y $r_{2}$. A diferencia del operador shift, este asegura un intercambio simultáneo de clientes. El número de clientes a transferir están definidos por dos valores de $\lambda \leq 2$ de la forma $\operatorname{swap}\left(\lambda_{1}, \lambda_{2}\right)$. Esto permite una adecuada cantidad de clientes transferidos entre rutas con un amplio margen de exploración. La función $\operatorname{swap}\left(\lambda_{1}, \lambda_{2}\right)$ se define en los vecindarios $N L=\left[N^{3}, N^{4}, N^{5}\right]$ de acuerdo a sus posibles combinaciones $\operatorname{con} \lambda \leq 2 \rightarrow\{(1,1),(2,2),(2,1)\}$.

- Operador $k$-shift: consiste en la transferencia de $k$ clientes consecutivos desde la ruta $r_{1}$ al final de la ruta $r_{2}$. El valor de $k$ puede variar dependiendo de la cantidad de clientes del problema, ya que valores muy grandes tienden a ser infactibles. Por obvias razones, este valor será $K \geq 3$ ya que la función shift tiene valores $\lambda=2$. Con respecto a las instrucciones requeridas para construir el algoritmo que efectúe la función $k$-shift, son las mismas de la función shift $(\lambda, 0)$ con la diferencia de que el espacio de transferencia está al final de la ruta, es decir, es un operador con una complejidad computacional más baja que los otros operadores inter-ruta que generan vecindarios. Esta función ocupa los vecindarios $N L=\left[N^{6}, N^{7}, N^{8}\right]$ de acuerdo a sus posibles combinaciones con $\lambda \leq 2 \rightarrow\{(3,0),(4,0),(5,0)\}$.

\subsection{Operadores de intercambio intra-ruta}

En la búsqueda local, es implementada una etapa de mejoramiento a través de la aplicación de operadores de intercambio intra-ruta. Para esto, son definidos cinco operadores. Su característica es realizar movimientos en una misma ruta. Estos operadores se les denomina «estructuras de mejoramiento posteriores a las búsquedas interruta». Estos operadores solo minimizan la función objetivo, asociada a la distancia recorrida por las rutas, como el costo. Las emisiones de $\mathrm{CO}_{2}$ son reducidas proporcionalmente a la distancia ${ }^{1}$ y el valor de equidad no será afectado significativamente al permanecer constante la cantidad de clientes. Una ventaja de estos operadores es que sus movimientos no producen infactibilidad en los vehículos. Se define que los movimientos intra-ruta de cada operador se realizan con mejoramientos secuenciales; es decir, si un movimiento realizado mejora la solución, no se reinician los intercambios, se seguirán buscando mejores soluciones en iteraciones siguientes del mismo operador, a diferencia de los operadores inter-ruta.

Los operadores utilizados son descritos así:

- Operador OR-OPT: este operador selecciona un cliente que se va a remover de su posición actual para ser insertado en cualquier otra posición de la ruta. Este movimiento se realiza para cada uno de los nodos.

${ }^{1}$ El frente de Pareto es generado en el GVRPPWE debido al uso de vehículos subcontratados sin arco de retorno al depósito. Dichos vehículos aumentan el costo, pero disminuyen las emisiones. 
- Operador OR-OPT2: dos nodos adyacentes cambian de posición en la ruta.

- Operador OR-OPT3: tres nodos adyacentes son ubicados en otra posición de la ruta.

- Operador 2-OPT: este operador ejecuta un movimiento que consiste en remover dos arcos no adyacentes e ingresarlos nuevamente de forma cruzada.

- Operador Exchange: este operador realiza un movimiento de permutación de posición entre dos nodos.

\subsection{Perturbación}

La perturbación es el mecanismo que permite la exploración en diversos espacios de la solución y consiste en aplicar movimientos intra-ruta de manera aleatoria. A esto, se le llama diversificación. Estos movimientos permiten salir de óptimos locales y alcanzar otros puntos del espacio de solución. Los movimientos de perturbación son aplicados mediante la selección de operadores de manera aleatoria. Para la aplicación de cada operador, es necesario definir un grado de perturbación, el cual indica, básicamente, el número de movimientos intra-ruta que deben realizarse de forma secuencial, uno tras otro, aleatoriamente y sin la necesidad de mejorar la función objetivo. Este grado es controlado por el vector $P$ que es definido en Subramanian (2012) y se describe en 5.6:

$$
P=[0.5 v, 0.6 v, 0.7 v, 0.8 v, 0.91 v, 1.1 v, 1.2 v, 1.3 v, 1.4 v, 1.5 v, 1.6]
$$

Donde $v$ es el número total de vehículos que tiene la solución. Es importante tener en cuenta que la limitación de esta perturbación establece que la transferencia de nodos entre rutas debe ser de forma tal que las dos rutas sigan siendo factibles.

Para el procedimiento de perturbación, son definidas dos clases de estructuras basadas en los operadores de intercambio intra-ruta. Estas son descritas a continuación:

- MULTIPLE-SHIFT $(1,1)$ : Es un operador de intercambio no simultáneo (pero aleatorio) de dos clientes entre un par de rutas. Por ejemplo, para las rutas $r_{1}=[1,2,3,4,5]$ y $r_{2}=[6,7,8,9,10]$, si aleatoriamente se seleccionaran los clientes $[3,7]$, primero el cliente 3 es insertado en la ruta $r_{2}$ en una posición aleatoria, siempre que sea factible. Posteriormente, se realiza el mismo procedimiento para el cliente 7 . Finalmente, las rutas podrían quedar así: $r_{1}=[1,2,4,7,5]$ y $r_{2}=[3,6,8,9,10]$. No es necesario que el primer movimiento sea factible para que se realice el segundo o viceversa.

- MULTIPLE-SWAP $(1,1)$ : A diferencia del otro operador, los intercambios de perturbación deben realizarse de forma simultánea, esto significa que los clientes transferidos intercambian posiciones. Así, ambos son factibles. 
Por ejemplo, para las rutas $r_{1}=[1,2,3,4,5]$ y $r_{2}=[6,7,8,9,10]$, si aleatoriamente se seleccionaran los clientes $[3,7]$, se deberá verificar si es factible la transferencia de ambos clientes. Posteriormente, se realiza el movimiento simultáneo de intercambio. Las rutas quedarían así $r_{1}=[1,2,7,4,5]$ y $r_{2}=[6,3,8,9,10]$.

\subsubsection{Criterio de distancia en movimientos de perturbación}

Los procedimientos de perturbación pueden hacer que la solución que se utiliza para realizar las búsquedas locales no sea la adecuada para vecindarios relativamente cercanos. Esto afecta el desempeño del algoritmo ILS ya que, al aumentar la diversificación, se pierde intensificación en la búsqueda. Para balancear estos aspectos, es implementado un criterio de distancia que limita los movimientos de perturbación.

Este criterio consiste en generar una distancia desde un punto de referencia de una ruta y un nodo candidato a transferirse o intercambiarse. Para ello, se determina el centroide de cada ruta. La distancia de referencia será calculada entre el centroide de la ruta y el depósito. Se define que en el centroide de cada ruta existirá un radio que dibuja una circunferencia y los nodos de otras rutas que se encuentren al interior de esta circunferencia son denominados «perturbables a la ruta», es decir, se podrán transferir o intercambiar. El centroide se calcula como la distancia promedio de cada punto de la ruta al depósito. En la Figura nro. 22, se muestra un ejemplo con los centroides y la circunferencia, los cuales limitan los nodos que pueden incorporarse a la ruta. El radio de la circunferencia es variable al ser multiplicado por el factor de distancia. Este permite controlar el vecindario de clientes que pueden transferirse.

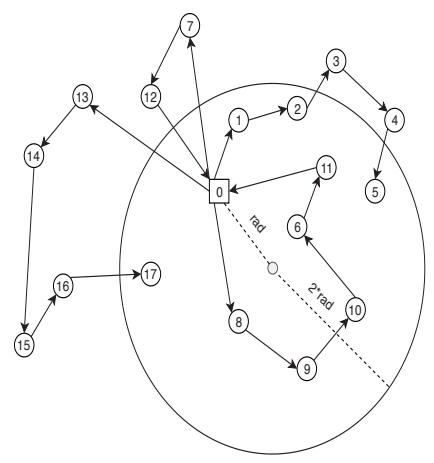

Figura NRO. 22. Criterio de distancia.

Elaboración propia.

Inicialmente, son seleccionados los operadores de perturbación y las rutas que serán perturbadas para cada uno de estos. De las rutas, son identificados los nodos a transferir. El número de configuraciones vecinas 
son restringidas, esto ayuda a reducir la complejidad computacional del problema. No obstante, a pesar de que se seleccionen nodos que son perturbables, no significa que vayan a ser transferidos, ya que es necesario que su movimiento sea factible en términos de la carga disponible de los vehículos.

\subsection{Selección de soluciones no dominadas}

El concepto de dominancia es definido en detalle en la sección 2.6.1. Este se relaciona con la viabilidad que tiene una solución encontrada de ingresar al frente de Pareto. Cada ciclo de búsqueda del algoritmo ILS genera una nueva solución candidata y se debe determinar si esta solución obtenida puede ingresar al Pareto. Con esa meta, se efectúan los siguientes procedimientos:

1. Es verificada la región de dominancia de la solución candidata para ingresar al Pareto. Allí, no debe existir ninguna solución en el Pareto que la domine. En caso de que sea cierto, la solución podrá ingresar al Pareto; de lo contrario, se descarta.

2. Si la solución ingresó al Pareto, se realiza una verificación de su zona inferior. Todas las soluciones que se ubiquen en esta región serán eliminadas del Pareto debido a que serían dominadas por la nueva solución que ingresa.

En el algoritmo 4 se muestra el pseudocódigo del procedimiento de selección de soluciones no dominadas.

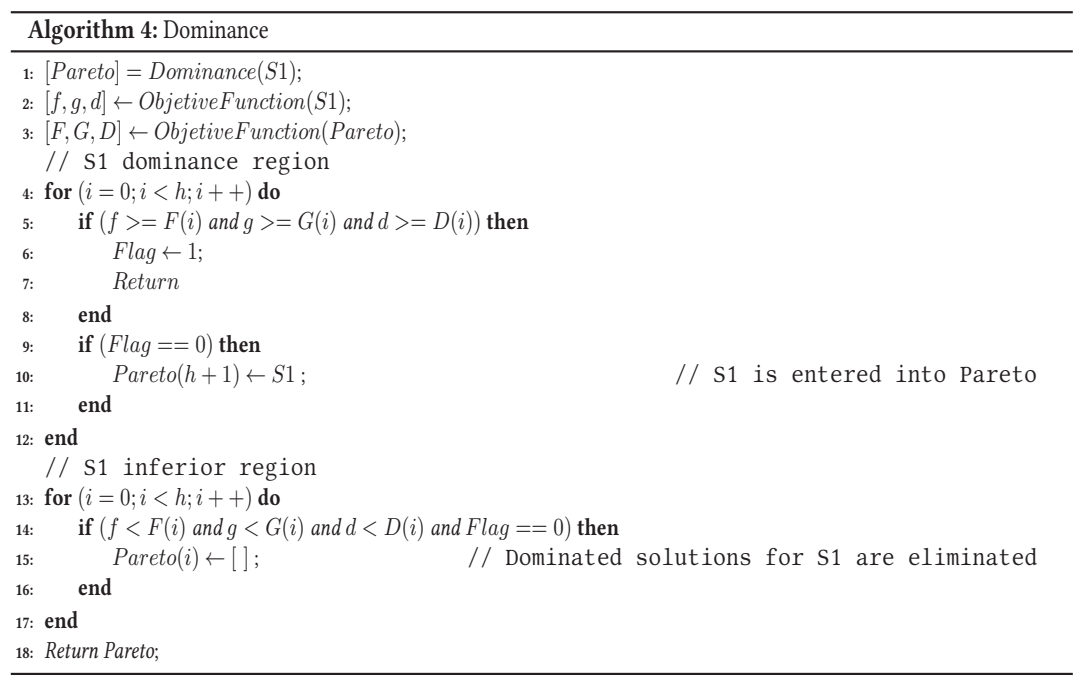




\subsubsection{Descripción pseudocódigo: dominancia}

En la linea 1 es definida la función que es usada en el algoritmo ILS (algoritmo 1, línea 24). En las líneas 2 y 3, se obtienen los valores de funciones objetivo de la solución $S 1$ la cual es candidata a ingresar al Pareto y los vectores con los valores de las funciones objetivo de todo el Pareto, respectivamente. Entre las líneas 4 y 11, se verifica la región de dominancia; si no existe ninguna otra solución en esta región, esto indica que la solución no está dominada por ninguna otra existente en el Pareto; por lo tanto, puede ingresar a este, como se muestra en la línea 10. Entre las líneas 13 y 17, se realiza la verificación de la región inferior en la solución candidata $S 1$. Si existe alguna solución que esta domine después de ingresar al Pareto, será eliminada, como se muestra en la línea 15.

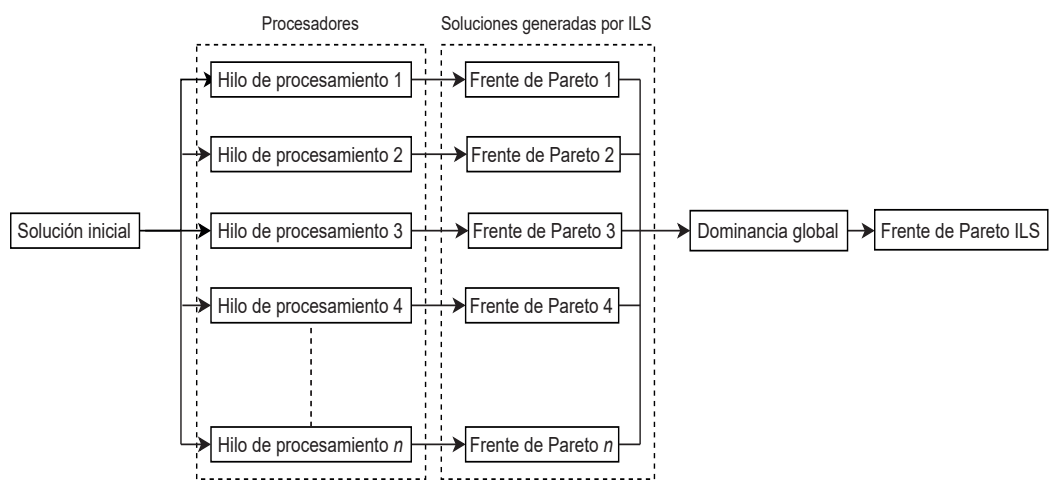

FiguRA NRO. 23. Esquema procesamiento paralelo.

Elaboración propia.

\subsection{Procesamiento en paralelo}

En esta implementación, el procesamiento en paralelo es una herramienta importante para la construcción de frentes de Pareto densos en menos tiempo computacional, ya que, de esta forma, se usa toda la capacidad de procesamiento disponible para la construcción del Pareto. El paralelismo en el procesamiento consiste en generar procesos de ejecución independientes a partir de la misma solución inicial, constituyendo diversos frentes de Pareto que deberán ser unificados con el fin de obtener un único frente. Para realizar la unificación de los Paretos, es necesario aplicar un procedimiento de dominancia global que consiste en unificar todos los Paretos y aplicar la función de dominancia, descrita en la sección 2.6.1. Al final de la ejecución, solo quedara un Pareto compuesto de soluciones no dominadas. La densidad del frente de Pareto opera como función de la profundidad de la búsqueda local definida. Entre 
Introducción al problema de enrutamiento de vehículos en la logística de distribución

mayor número de iteraciones se definan para la búsqueda local y el número de procesos en paralelo, el frente será más denso. En la Figura nro. 23 se muestra el esquema de procesamiento en paralelo, utilizado en este trabajo. 


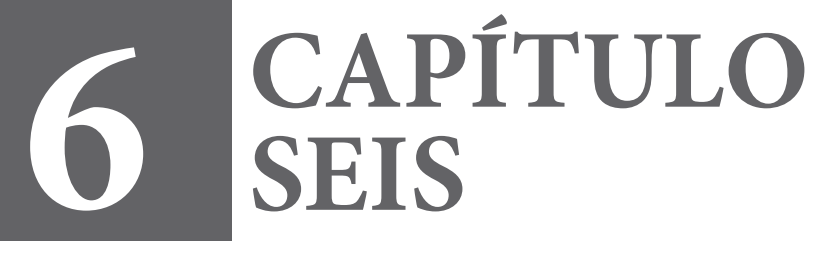




\section{RESULTADOS COMPUTACIONALES}

Los modelos matemáticos exactos, asociados a los problemas descritos entre las secciones 6.1 y 6.4, fueron implementados en AMPL: A Modeling Language for Mathematical Programming (Fourer et al., 1990) y resueltos con CPLEX 12.5 (IBM-ILOG, 2009), usando un computador Intel Core i7-4770 3.4 GHz de 4 GB de RAM.

Para la metodología aproximada, descrita en la sección 6.5, se implementó un algoritmo en C++ (Compilador: Microsoft Visual C++, 2015). Para esta codificación, se usaron herramientas de Matlab ${ }^{\odot}$, el paquete Coder $^{\odot}$ que permite la creación de código en $\mathrm{C}++$ a partir del construido en $\mathrm{Matlab}^{\odot}$, y el paquete Parallel Computing Toolbox ${ }^{\odot}$ para la implementación del procesamiento en paralelo. El equipo usado para las ejecuciones utiliza el sistema operativo Microsoft Windows 10 de 64 bits y un procesador Intel ${ }^{\circledR}$ Xeon $^{\oplus}$ CPU ES-2683 v4 @ 2.10 GHz, 16 procesadores y una memoria RAM de $64 \mathrm{~GB}$.

\subsection{Resultados de versiones VRP con flota propia y subcontratada}

Un número considerable de casos son implementados en los modelos con el fin de analizar el desempeño de la metodología propuesta en la solución del CLRPPC, VRPPC y MDVRPPC. El objetivo es observar la capacidad del modelo matemático propuesto para resolver diferentes tipos de problemas. Tres conjuntos de instancias de prueba son usados para realizar la validación, uno para cada variante. Los casos van de 20 a 50 clientes y de 1 a 5 depósitos. En las instancias implementadas, son descritas las principales características que dependen del problema analizado y son descritas en general, como: nombre de la instancia o caso, cantidad de depósitos, costo asociado a los recorridos de los vehículos e identificación de las rutas propias y subcontratadas, costos fijos por uso de vehículos y depósitos, tiempo requerido para cada instancia, función objetivo y GAP en porcentaje. En todos los casos, los arcos o trayectos entre nodos, recorridos por vehículos subcontratados, fueron penalizados con un valor $P=2$ con respecto a los recorridos de los vehículos propios. 
Tanto para el MDVRPPC como para el VRPPC en la función objetivo solo se consideran los costos de uso de arcos por la flota propia o por la subcontratada; en estos problemas, no se consideran costos por apertura de depósitos ni costos asociados a la utilización de vehículos. Las instancias usadas para el CLRPPC fueron tomadas de Prins et al. (2007) y se adaptaron al problema en particular. Para esto, fue necesario definir el número de vehículos disponibles $k$, obtenido a través de la ecuación 6.1, con base en la propuesta de Potvin y Naud (2011), donde $q$ denota la demanda total del conjunto de clientes y $Q$ corresponde a la capacidad máxima de carga que un vehículo de flota homogénea puede transportar.

$$
k=0.8 q / Q \text { (Número máximo de vehículos de la flota propia.) }
$$

En la Tabla nro. 9, son presentados los resultados obtenidos para el CLRPPC, donde $k$ representa el número de vehículos de la flota propia disponibles. «F. O.» denota la función objetivo. En dicha tabla se consideran los costos de apertura de los $\mathrm{CD}$, los costos asociados al desplazamiento entre de los arcos por la flota propia o subcontratada y los costos fijos asociados a la utilización de los vehículos propios, tomados de Prins et al. (2007). El tiempo requerido por el software para resolver las instancias de prueba es dado en segundos. El GAP denota la diferencia entre los límites superiores e inferiores, entregados por el CPLEX. En la Tabla nro. 10 se presentan las rutas obtenidas para cada solución.

\begin{tabular}{|c|c|c|c|c|c|c|c|}
\hline Caso & $k$ & Costo-CDs & Costos rutas & $\begin{array}{l}\text { Costo por uso de } \\
\text { vehículos propios }\end{array}$ & F.O. & $\begin{array}{l}\text { tiempo } \\
\text { (s) }\end{array}$ & $\begin{array}{l}\text { GAP } \\
(\%)\end{array}$ \\
\hline $20-5-1 a$ & 3 & 25549 & $\begin{array}{r}\text { Propias: } 10340 \\
\text { Sub: } 16664\end{array}$ & 1000 & 53553 & 7201 & 0 \\
\hline $20-5-1 b$ & 3 & 15497 & $\begin{array}{r}\text { Propias: } 16920 \\
\text { Sub: } 3734\end{array}$ & 2000 & 38151 & 24 & 0 \\
\hline $20-5-2 a$ & 3 & 24156 & $\begin{array}{r}\text { Propias: } 5965 \\
\text { Sub: } 16240\end{array}$ & 1000 & 47401 & 3000 & 0 \\
\hline $20-5-2 b$ & 3 & 13911 & $\begin{array}{r}\text { Propias: } 18761 \\
\text { Sub: } 1922\end{array}$ & 3000 & 36594 & 15 & 0 \\
\hline 50-5-2bBIS & 3 & 18763 & $\begin{array}{r}\text { Propias: } 3450 \\
\text { Sub: } 27906\end{array}$ & 1000 & 51119 & 80000 & 5 \\
\hline
\end{tabular}

TABla NRo. 9. Resultados CLRPPC.

Elaboración propia.

Los resultados muestran un buen desempeño del modelo matemático asociado al GAP, obtenido para cada instancia estudiada, que va de 20 a 50 clientes. En estos casos, los valores de GAP son iguales a 0 en los casos de 20 clientes y del $5 \%$ la instancia de 50 clientes. En esa dirección, los tiempos computacionales son considerados como aceptables.

En la Tabla nro. 11 se presentan los resultados obtenidos para la implementación del MDVRPPC. Las 


\begin{tabular}{llll}
\hline Caso & $k$ & Rutas Propias & Rutas Subcontratadas \\
\hline 20-5-1a & 3 & Ruta 1: 3-24-16-19-8-12-3 & Sub 1: 2-9-6-17 \\
& & & Sub 2: 2-10-18-23-25 \\
& & & Sub 3: 3-13-11 \\
& & & Sub 4: 5-15-14-22-7 \\
& & & Sub 5: 5-21-20 \\
\hline 20-5-1b & 3 & Ruta 1: 3-22-6-23-7-15-21-20-12-25-3 & Sub 1: 3-8-13-24 \\
& & Ruta 2: 4-17-18-10-19-16-9-14-11-4 & \\
\hline 20-5-2a & 3 & Ruta 1: 4-12-7-14-8-4 & Sub 1: 1-6 \\
& & & Sub 2: 1-9-11 \\
& & & Sub 3: 1-15 \\
& & & Sub 4: 4-10-18 \\
& & & Sub 5: 4-13 \\
& & & Sub 6: 5-21-16 \\
& & & Sub 7: 5-23-20-17-24 \\
& & & Sub 8: 5-25-19-22 \\
\hline 20-5-2b & 3 & Ruta 1: 2-18-19-23-25-21-22-24-16-17-2 & Sub 1: 4-15-8-14 \\
& & Ruta 2: 4-6-11-9-7-10-13-20-12-4 & \\
\hline 50-5-2bBIS & 3 & Ruta 1: 4-24-26-28-23-17-13-20-14-15-4 & Sub 1: 2-30-18-21-8-12-6 \\
& & & Sub 2: 2-55-49-41-37-51-38-34-42-52-36 \\
& & & Sub 3: 4-11-16-19-22-29-25-10-27-9-7 \\
& & & Sub 4: 5-45-35-40-47-53-33 \\
& & & Sub 5: 5-48-39-50-32-44-43-46-31-54 \\
\hline
\end{tabular}

TABLA NRO. 10. Rutas de los vehículos del CLRPPC.

Elaboración propia.

\begin{tabular}{|c|c|c|c|c|c|c|}
\hline Caso & $k$ & \multicolumn{2}{|c|}{ Costos rutas } & F. 0 . & tiempo & GAP \\
\hline & & | Propias | & Subcontratadas $\mid$ & & $(\mathrm{s})$ & $(\%)$ \\
\hline P01-20-4 & 4 & 218 & 82 & 300 & 212 & 0 \\
\hline P01-25-4 & 4 & 268 & 100 & 368 & 27047 & 0 \\
\hline P01-30-4 & 5 & 315 & 96 & 411 & 42894 & 2 \\
\hline P01-30-3 & 5 & 344 & 94 & 438 & 61809 & 2 \\
\hline P02-50-4 & 4 & 383 & 138 & 521 & 50000 & 6 \\
\hline
\end{tabular}

TABLA NRO. 11. Resultados MDVRPPC.

Elaboración propia.

instancias estudiadas PO1 y PO2 fueron obtenidas de Cordeau et al. (1997). PO1 y PO2 fueron modificadas para generar instancias adecuadas para implementar el problema. Así, el caso P01-20-4 considera 4 depósitos y los 20 primeros clientes. La función objetivo (F. O.) denota la sumatoria de los costos asociados a los arcos recorridos por la flota propia o subcontratada. La ruta subcontratada considera una penalidad de 2 con respecto a la propia. Para casos de 20 y 30 clientes, los valores de GAP se encontraron en un rango entre $0-2 \%$. Los tiempos computacionales son considerados como aceptables. En la Tabla nro. 12, se presentan las rutas obtenidas de las flotas propias y subcontratadas.

En la Tabla nro. 13, se presentan los resultados obtenidos en relación con el VRPPC. Para este tipo de problemas, se usaron instancias del CVRP de 16 a 50 clientes, obtenidas de Augerat et al. (1998) y adaptadas por medio de la ecuación 6.1. La F. O. considera los costos asociados de los recorridos entre los arcos por la flota propia o subcontratada. De la misma forma que, para el CLRPPC y MDVRPPC, se usa una penalidad $P=2$; se usa para los arcos de la flota subcontratada. Los valores obtenidos de GAP son del $0 \%$ para las instancias de hasta 23 clientes 
Introducción al problema de enrutamiento de vehículos en la logística de distribución

y entre el $1 \%$ y $5 \%$ para las instancias de mayor tamaño. Asimismo, los tiempos computacionales son aceptables. En la Tabla nro. 14 son presentadas las rutas propias y subcontratadas, obtenidas por cada instancia. Los resultados asociados al GAP permiten concluir que la metodología tiene un buen desempeño.

\begin{tabular}{llll}
\hline Caso & $k$ & Rutas propias & Rutas subcontratadas \\
\hline P01-20-4 & 4 & Ruta 1: 1-23-17-1 & Sub 1: 1-8-22-18 \\
& & Ruta 2: 2-10-11-12-5-2 & Sub 2: 4-24-7 \\
& Ruta 3: 3-14-19-21-16-9-3 & \\
\hline & Ruta 4: 3-15-6-20-13-3 & Sub 1: 1-8 \\
\hline P01-25-4 & 4 & Ruta 1: 1-22-17-23-1 & Sub 2: 2-10-18-29 \\
& & Ruta 2: 2-28-27-11-12-5-2 & Sub 3: 4-24-7 \\
& & Ruta 3: 3-14-19-21-16-9-13-3 & \\
\hline P01-30-4 & Ruta 4: 4-6-26-15-20-25-4 & Ruta 1: 1-23-17-22-21-1 & Sub 1: 1-8 \\
& & Ruta 2: 2-5-26-12-30-11-27-2 & Sub 2: 2-16 \\
& & Ruta 3: 2-10-28-29-18-2 & Sub 3: 2-31 \\
& & Ruta 4: 3-9-19-14-34-13-3 & Sub 4: 4-24-7-32 \\
& Ruta 5: 4-33-6-15-20-25-4 & \\
\hline P01-30-3 & Ruta 1: 1-22-16-21-20-1 & Sub 1: 1-7 \\
& Ruta 2: 2-23-6-31-25-4-2 & Sub 2: 2-15 \\
& Ruta 3: 2-27-26-10-29-11-2 & Sub 3: 2-30-9-17-28 \\
& Ruta 4: 3-8-18-13-33-12-3 & \\
\hline P02-50-4 & Ruta 5: 3-14-5-32-24-19-3 & Ruta 1: 1-8-51-22-17-45-23-44-46-1 & Sub 1: 2-31-10-18-29 \\
& Ruta 2: 2-52-27-28-47-11-30-12-35-32-26-5-36-50-2 & Sub 2: 4-24-7-39-40 \\
& Ruta 3: 3-13-34-38-25-54-20-6-15-42-3 & Sub 3: 4-33 \\
& Ruta 4: 3-14-43-37-49-19-48-41-21-16-9-53-3 & \\
\hline
\end{tabular}

TABLA NRO. 12. Rutas de los vehículos del MDVRPPC.

Elaboración propia.

En las figuras 24 y 25, se presentan como ejemplo de soluciones óptimas, las obtenidas para el CLRPCC-505-2bBIS y para el caso de único depósito identificado como CVRPPC A-n39-k6 .

\subsection{Resultados de las versiones VRP sin retorno al depósito}

\subsubsection{Resultados OLRP}

Tres conjuntos de prueba son usados con el fin de validar la operación y efectividad propuesta en esta formulación, donde los dos primeros conjuntos corresponden a casos del OLRP: el primer conjunto de datos se relaciona con 30 casos, adaptados de Prins et al. (2006); el segundo conjunto de casos de prueba está conformado por 36 casos, adaptados de Tuzun y Burke (1999). Los resultados del OLRP son comparados con los publicados por Yu y Lin (2015). El tercer conjunto contiene 24 casos MDOVRP, los cuales son usados por Liu y Jiang (2012) y Lalla-Ruiz et al. (2016), tomado de Cordeau et al. (1997).

\begin{tabular}{ccll}
\hline Caso & $k$ & Rutas propias & Rutas subcontratadas \\
\hline P-n16-k8 & 6 & Ruta 1: 1-5-12-1 & Sub 1: 1-2-4
\end{tabular}




\begin{tabular}{|c|c|c|c|}
\hline \multirow[t]{6}{*}{ Caso } & $k$ & Rutas propias & Rutas subcontratadas \\
\hline & & Ruta 2: 1-9-1 & Sub 2: 1-7 \\
\hline & & Ruta 3: 1-14-10-8-1 & \\
\hline & & Ruta 4: 1-16-13-11-1 & \\
\hline & & Ruta 5: 1-15-6-1 & \\
\hline & & Ruta 6: 1-3-1 & \\
\hline P-n19-k2 & 1 & Ruta 1: 1-2-11-5-12-15-13-4-18-17-9-6-1 & Sub 1: 1-7-19-3-8-10-16-14 \\
\hline P-n20-k2 & 1 & Ruta 1: 1-2-11-14-9-18-19-4-13-16-12-5-1 & Sub 1: 1-7-20-6-15-17-10-8-3 \\
\hline P-n21-k2 & 1 & Ruta 1: 1-5-12-16-13-4-20-19-9-11-2-17-1 & Sub 1: 1-7-21-6-15-18-10-14-8-3 \\
\hline P-n22-k2 & 1 & Ruta 1: 1-2-11-5-12-16-13-4-20-19-9-14-10-1 & Sub 1: 1-17-7-21-6-15-18-22-8-3 \\
\hline \multirow[t]{5}{*}{ P-n22-k8 } & 5 & Ruta 1: 1-10-3-2-7-1 & Sub 1: 1-8-6 \\
\hline & & Ruta 2: 1-11-9-4-5-1 & Sub 2: 1-13 \\
\hline & & Ruta 3: 1-14-12-1 & Sub 3: 1-17 \\
\hline & & Ruta 4: 1-15-21-22-1 & Sub $4: 1-20$ \\
\hline & & Ruta 5: 1-16-19-18-1 & \\
\hline \multirow[t]{6}{*}{ P-n23-k8 } & 6 & Ruta 1: 1-2-3-1 & Sub 1: 1-22-7-21 \\
\hline & & Ruta 2: 1-4-20-19-1 & Sub 2: 1-17-9 \\
\hline & & Ruta 3: 1-8-5-1 & \\
\hline & & Ruta 4: 1-11-13-16-12-1 & \\
\hline & & Ruta 5: 1-18-10-14-1 & \\
\hline & & Ruta 6: 1-23-15-6-1 & \\
\hline \multirow[t]{4}{*}{ P-n40-k5 } & 4 & Ruta 1: 1-7-25-24-8-27-9-32-29-2-28-1 & Sub 1: 1-13-6-39-10 \\
\hline & & Ruta 2: 1-15-26-14-20-5-19-1 & \\
\hline & & Ruta 3: 1-18-38-16-34-40-11-31-35-22-30-17-1 & \\
\hline & & Ruta 4: 1-3-33-23-4-37-36-21-3-12-1 & \\
\hline \multirow[t]{4}{*}{ P-n45-k5 } & 4 & Ruta 1: 1-5-14-42-20-41-43-45-38-6-1 & Sub 1: 1-13-19-15-26 \\
\hline & & Ruta 2: 1-7-24-25-44-8-27-32-29-9-28-1 & \\
\hline & & Ruta 3: 1-12-3-30-21-36-37-4-23-2-33-1 & \\
\hline & & Ruta 4: 1-18-16-34-40-11-31-35-22-17-10-39-1 & \\
\hline \multirow[t]{5}{*}{ P-n50-k7 } & 5 & Ruta 1: 1-8-36-20-15-12-39-27-1 & Sub 1: 1-5-35-47-9 \\
\hline & & Ruta 2: 1-13-11-32-26-19-25-45-4-1 & Sub 2: 1-18-41-33-10-40 \\
\hline & & Ruta 3: 1-17-50-24-44-42-43-23-2-34-7-1 & \\
\hline & & Ruta 4: 1-28-14-16-21-38-37-6-30-46-1 & \\
\hline & & Ruta 5: 1-31-49-48-22-29-3-1 & \\
\hline \multirow[t]{3}{*}{ A-n32-k5 } & 3 & Ruta 1: 1-7-4-3-24-5-12-29-15-1 & Sub 1: 1-25-28 \\
\hline & & Ruta 2: 1-27-8-14-18-20-32-22-1 & Sub 2: 1-31-17-13-2 \\
\hline & & Ruta 3: 1-30-19-9-10-23-16-11-26-6-21-1 & \\
\hline \multirow[t]{3}{*}{ A-n33-k5 } & 3 & Ruta 1: 1-21-6-27-8-9-14-33-3-1 & Sub 1: 1-5-13-11-31-26-28 \\
\hline & & Ruta 2: $1-25-7-20-15-22-2-32-12-1$ & Sub 2: $1-23$ \\
\hline & & Ruta 3: 1-30-17-4-10-18-16-1 & Sub 3: 1-24-29-19 \\
\hline \multirow[t]{4}{*}{ A-n39-k6 } & 4 & Ruta 1: 1-19-28-11-17-5-9-8-1 & Sub 1: 1-16-14-31 \\
\hline & & Ruta 2: 1-21-33-35-23-22-24-18-37-2-7-1 & Sub 2: 1-27-12 \\
\hline & & Ruta 3: 1-25-4-39-13-10-29-30-6-1 & \\
\hline & & Ruta 4: 1-38-32-15-36-26-34-20-3-1 & \\
\hline
\end{tabular}

TABLA NRO. 14. Rutas de los vehículos del VRPPC. Elaboración propia.

En la Tabla nro. 15, se presentan los resultados comparativos con el estado del arte para el OLRP. Para cada 


\begin{tabular}{|c|c|c|c|c|c|c|c|c|}
\hline \multirow[t]{2}{*}{ Caso } & \multirow[t]{2}{*}{$k$} & \multicolumn{2}{|r|}{ Rutas } & \multicolumn{2}{|c|}{ Costos rutas } & \multirow[t]{2}{*}{ F.O. } & \multirow{2}{*}{$\begin{array}{c}\text { tiempo } \\
\text { (seg) }\end{array}$} & \multirow{2}{*}{$\frac{\text { GAP }}{(\%)}$} \\
\hline & & |Propias & |Subcontratadas & Propias & Subcontratadas & & & \\
\hline P-n16-k8 & 6 & 6 & 2 & 360 & 90 & 450 & 4 & 0 \\
\hline P-n19-k2 & 1 & 1 & 1 & 138 & 116 & 254 & 57 & 0 \\
\hline P-n20-k2 & 1 & 1 & 1 & 128 & 138 & 266 & 167 & 0 \\
\hline P-n21-k2 & 1 & 1 & 1 & 117 & 150 & 267 & 404 & 0 \\
\hline P-n22-k2 & 1 & 1 & 1 & 139 & 132 & 271 & 312 & 0 \\
\hline P-n22-k8 & 6 & 5 & 4 & 413 & 176 & 589 & 2418 & 0 \\
\hline P-n23-k8 & 6 & 5 & 2 & 430 & 102 & 532 & 8000 & 0 \\
\hline P-n40-k5 & 4 & 4 & 1 & 416 & 62 & 478 & 55165 & 3 \\
\hline P-n45-k5 & 4 & 4 & 1 & 464 & 76 & 540 & 68890 & 4 \\
\hline P-n50-k7 & 5 & 5 & 2 & 466 & 112 & 578 & 140375 & 5 \\
\hline A-n32-k5 & 3 & 3 & 2 & 652 & 152 & 804 & 30500 & 5 \\
\hline A-n33-k5 & 3 & 3 & 2 & 482 & 204 & 686 & 40750 & 1 \\
\hline A-n39-k6 & 4 & 4 & 2 & 741 & 96 & 837 & 53500 & 2 \\
\hline
\end{tabular}

TABLA NRO. 13. Resultados VRPPC

Elaboración propia.

instancia, la tabla entrega el mejor límite inferior conocido $(L B=$ LowerBound $)$, el valor relativo del GAP calculado porcentualmente y el tiempo computacional. Para el método propuesto, los valores presentados en negrilla, debajo de la columna de CPLEX, muestran los casos que han sido alcanzados o aquellos a los cuales se les ha mejorado la función objetivo, en comparación con la mejor reportada en la literatura. Esa misma información, se remarca debajo de la columna denominada $\operatorname{Gap}(\%)^{d}$, donde los valores negativos indican los casos que han mejorado el $\mathrm{BKS}^{A}$ (mejor solución obtenida usando el algoritmo SA). En la columna $\operatorname{Gap}(\%)^{c}$, los valores en negrilla indican los mejores GAP reportados usando un método matemático exacto. Finalmente, los números en negrilla de la columna $\operatorname{Gap}(\%)^{e}$ muestran la mejor solución obtenida por una técnica exacta. Como se puede ver en la Tabla nro. 15, el modelo produce resultados de alta calidad; por ejemplo, se obtienen las mejores soluciones exactas en 29 de los 30 problemas evaluados. Para 8 casos, se encuentran mejores resultados, comparados con los hallados por el método SA.

Los casos de 20 y 50 clientes tienen un límite de tiempo de 900 segundos y los restantes de 14400 segundos. La solución media obtenida por el modelo propuesto es 169 377.53, mientras que la solución obtenida por medio SA es 171 031.23. El rendimiento de la formulación matemática se puede comparar únicamente en los casos de 100 clientes o menos porque, para 200 clientes, no se reportan resultados. Para los casos de 200 clientes, el GAP se reporta por primera vez.

En la Tabla nro. 16 se presenta el mismo tipo de resultados respecto a la Tabla nro. 15. En ambos, los resultados reportados por Yu y Lin (2015) consideran un límite de tiempo de 14400 segundos y las corridas se realizaron en computadores con las mismas características. Todos los resultados reportados en Yu y Lin (2015), donde la solución fue obtenida con CPLEX, fueron superados por el modelo matemático propuesto en este trabajo. Finalmente, los límites inferiores prueban que son más fuertes que los presentados en Yu y Lin (2015). 


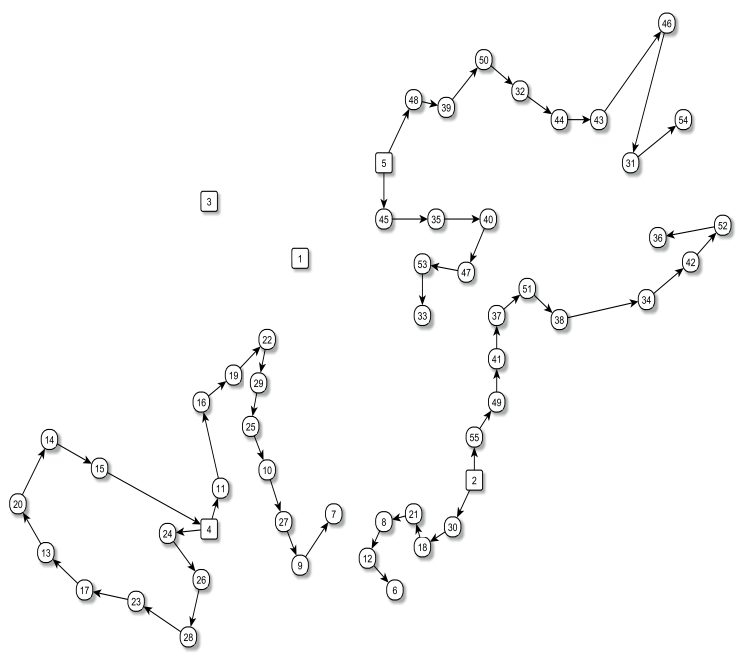

FiguRA NRO. 24. Solución óptima obtenida para el CLRPPC-50-5-2bbis.

Elaboración propia.

\subsubsection{Resultados MDOVRP}

En la Tabla nro. 17 se presentan resultados comparativos con métodos exactos de otros autores (Liu et al., 2014). Para cada modelo matemático, la tabla entrega la mejor solución calculada como el límite superior, el GAP relativo (en \%) y el tiempo computacional (en seg). Los resultados presentados por Liu et al. (2014) fueron ejecutados en un computador equipado con un procesador $3.2 \mathrm{GHz}$ Dual Core 4 GB de RAM y CPLEX 12.2, con un tiempo computacional máximo de 43200 segundos. Los resultados presentados por Lalla-Ruiz et al. (2016) se obtuvieron gracias al empleo de un equipo de cómputo Core $3.5 \mathrm{GHz}, 4$ GB de RAM y CPLEX versión 12.3, con un tiempo máximo de 7200 segundos. En este análisis, se considera un tiempo computacional de 7200 segundos para los casos p01 a p11. De la p12 en adelante, los resultados que se presentan fueron obtenidos en 14400 segundos.

Como se puede observar en los resultados de la Tabla nro. 17, el modelo que se propone produce resultados de alta calidad, obteniéndose iguales o mejores resultados en todos los casos y los límites inferiores presentados prueban ser mejores que los obtenidos por los otros dos métodos. El promedio de GAP es de $1.73 \%$ en comparación con los GAP de $62.03 \%$ y $7.77 \%$ reportados por otros autores en dos métodos. Para 12 instancias, se logra GAP de 0, de las cuales 4 nuevas soluciones óptimas se presentan para los casos p05, p06, p07, pr08 y pr09. La Figura nro. 26, se presenta como ejemplo de las soluciones óptimas obtenidas para pr08.

En la Tabla nro. 18 se presentan los resultados del MDOVRP con y sin restricción de distancia de las 
Introducción al problema de enrutamiento de vehículos en la logística de distribución

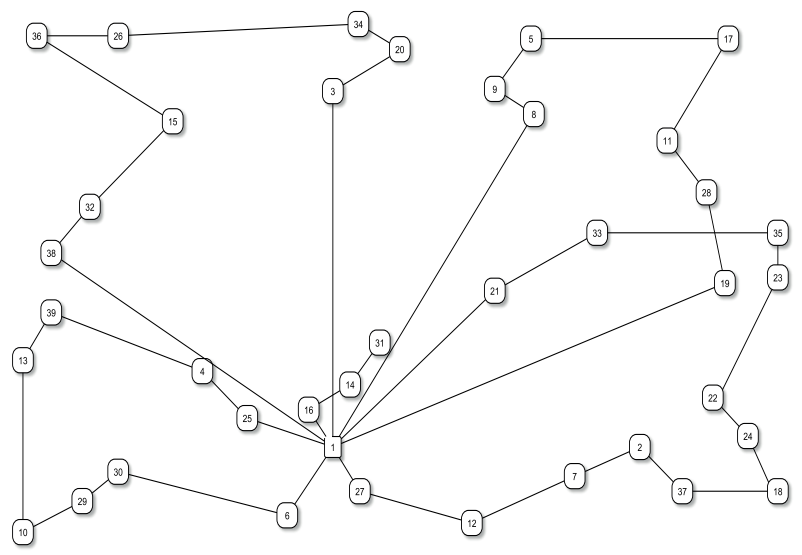

FIGURA NRO. 25. Solución óptima obtenida para el CVRPPC A-n39-k6. Elaboración propia.

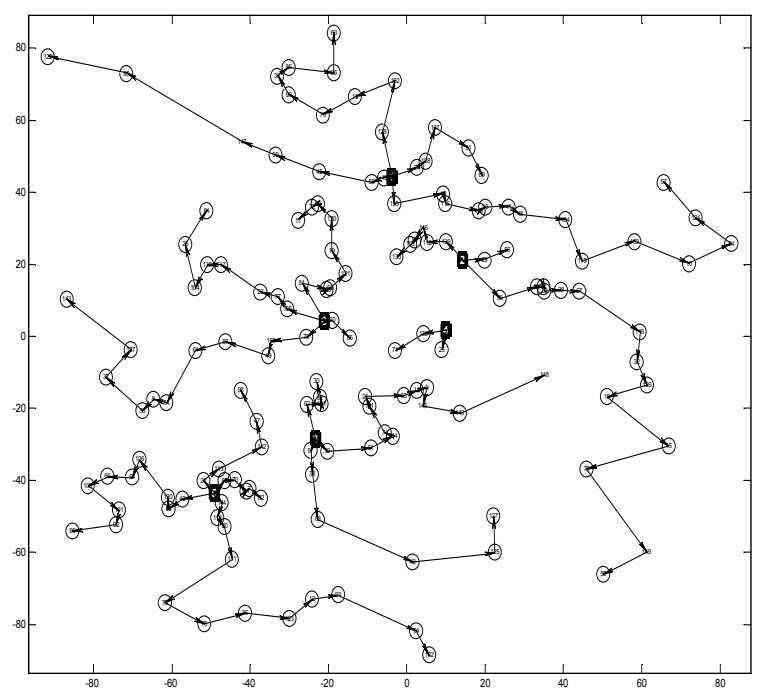

Figura NRO. 26. Solución óptima obtenida para pr08. Elaboración propia. 


\begin{tabular}{|c|c|c|c|c|c|c|c|c|c|}
\hline \multirow{3}{*}{ Caso } & \multicolumn{4}{|c|}{ Yu y Lin (2015) } & \multicolumn{5}{|c|}{ Modelo propuesto } \\
\hline & $\frac{\text { SA }}{\text { BKS }^{a}}$ & & CPLEX & $t(s)$ & CPIFX & & $\operatorname{Gan}(\sigma)^{d}$ & & $t(s)$ \\
\hline & & & Japt to & प(5) & CILE & & & & (s) \\
\hline $20-5-1-\mathrm{a}$ & 43849.00 & 43849.00 & 0.00 & 33.27 & 43849.00 & 0.00 & 0.00 & 0.00 & 5.00 \\
\hline $20-5-1-b$ & 33564.00 & 33564.00 & 0.00 & 2.36 & 33564.00 & 0.00 & 0.00 & 0.00 & 1.92 \\
\hline $20-5-2-a$ & 41125.00 & 41125.00 & 0.00 & 11.58 & 41125.00 & 0.00 & 0.00 & 0.00 & 5.56 \\
\hline $20-5-2-b$ & 32520.00 & 32520.00 & 0.00 & 1.53 & 32520.00 & 0.00 & 0.00 & 0.00 & 1.34 \\
\hline $50-5-1$ & 64217.00 & 64358.00 & 4.01 & 14400.00 & 64415.00 & 4.64 & 0.31 & 0.09 & 900.00 \\
\hline $50-5-1-b$ & 49114.00 & 49114.00 & 0.00 & 3400.00 & 49114.00 & 0.00 & 0.00 & 0.00 & 55.00 \\
\hline $50-5-2$ & 68121.00 & 68.121 .00 & 0.00 & 11.171 .00 & 68121.00 & 0.86 & 0.00 & 0.00 & 900.00 \\
\hline $50-5-2-b$ & 57355.00 & 57815.00 & 1.63 & 14400.00 & 57355.00 & 0.17 & 0.00 & -0.80 & 900.00 \\
\hline 50-5-2-bis & 60038.00 & 60052.00 & 3.64 & 14400.00 & 60038.00 & 3.23 & 0.00 & -0.02 & 900.00 \\
\hline 50-5-2-b-bis & 41193.00 & 41193.00 & 0.00 & 1608.00 & 41193.00 & 0.00 & 0.00 & 0.00 & 285.00 \\
\hline $50-5-3$ & 62581.00 & 62581.00 & 2.34 & 14400.00 & 62581.00 & 2.95 & 0.00 & 0.00 & 900.00 \\
\hline $50-5-3-b$ & 46584.00 & 46584.00 & 0.00 & 11238.00 & 46584.00 & 0.00 & 0.00 & 0.00 & 388.00 \\
\hline $100-5-1$ & 222634.00 & 228085.00 & 4.52 & 14400.00 & 222.819 .00 & 2.67 & 0.08 & -2.31 & 14400.00 \\
\hline $100-5-1-b$ & 189208.00 & 190417.00 & 2.68 & 14400.00 & 189.311 .00 & 2.36 & 0.05 & -0.58 & 14400.00 \\
\hline $100-5-2$ & 166328.00 & 167991.00 & 2.98 & 14400.00 & 167157.00 & 2.78 & 0.50 & -0.50 & 14400.00 \\
\hline $100-5-2-b$ & 144689.00 & 146668.00 & 2.38 & 14400.00 & 145279.00 & 1.31 & 0.41 & -0.95 & 14400.00 \\
\hline $100-5-3$ & 162746.00 & 252087.00 & 39.48 & 14400.00 & 163208.00 & 3.42 & 0.28 & -35.26 & 14400.00 \\
\hline $100-5-3-b$ & 134632.00 & 138364.00 & 4.34 & 14400.00 & 134795.00 & 1.25 & 0.12 & -2.58 & 14400.00 \\
\hline $100-10-1-a$ & 276859.00 & 308507.00 & 29.01 & 14400.00 & 243.116.00 & 4.02 & -12.19 & -21.20 & 14400.00 \\
\hline $100-10-1-b$ & 220134.00 & 273445.00 & 24.74 & 14400.00 & 210663.00 & 1.45 & -4.30 & -22.96 & 14400.00 \\
\hline $100-10-2$ & 213232.00 & 295374.00 & 33.85 & 14400.00 & 213548.00 & 2.26 & 0.15 & -27.70 & 14400.00 \\
\hline $100-10-2-b$ & 189818.00 & 260437.00 & 30.82 & 14400.00 & 190002.00 & 0.80 & 0.10 & -27.04 & 14400.00 \\
\hline $100-10-3$ & 214056.00 & 265158.00 & 27.66 & 14400.00 & 215368.00 & 5.46 & 0.61 & -18.78 & 14400.00 \\
\hline $100-10-3-b$ & 187792.00 & 197206.00 & 10.55 & 14400.00 & 187204.00 & 3.37 & -0.31 & -5.07 & 14400.00 \\
\hline $200-10-1$ a & 401.113 .00 & - & - & 14400.00 & 401.572 .00 & 9.40 & 0.11 & - & 14400.00 \\
\hline $200-10-1 \bar{b}$ & 342479.00 & - & - & 14400.00 & 342250.00 & 4.79 & -0.07 & - & 14400.00 \\
\hline $200-10-2 a$ & 398042.00 & - & - & 14400.00 & 390421.00 & 6.42 & -1.91 & - & 14400.00 \\
\hline $200-10-2 b$ & 351707.00 & - & - & 14400.00 & 350257.00 & 2.95 & -0.41 & - & 14400.00 \\
\hline $200-10-3$ & 391175.00 & - & - & 14400.00 & 391.206 .00 & 3.84 & 0.01 & - & 14400.00 \\
\hline $200-10-3 b$ & 324032.00 & - & - & 14400.00 & 322691.00 & 2.34 & -0.41 & - & 14400.00 \\
\hline Promedio & | 171031.23 & & & $11.460 .57 \mid$ & | 169377.53 & 2.40 & -0.56 & & 8814.73 \\
\hline
\end{tabular}

TABLA NRo. 15. Resultados computacionales del OLRP sobre los casos de Prins et al. (2006).

Elaboración propia.

rutas, planteados en la sección «Problema de enrutamiento multi-depósito abierto (MDOVRP)». Los resultados considerando el límite de distancia muestran que fue posible alcanzar el óptimo en las mismas instancias que en los casos sin límite de distancia, pero generalmente con GAP y tiempo de cómputo mayor. Dos casos especiales son: i) la instancia p07 cuya solución óptima es 614.09, pero solo se alcanzó gap=0 en 139838 segundos y ii) la instancia pr09 cuya solución óptima es 1594.01 se alcanzó gap=0 en 31516 segundos. El valor de distancia límite fue calculado a partir de la longitud de la ruta más larga obtenida en la solución del modelo sin límite de distancia. Así, por ejemplo, para la instancia p05 la longitud de ruta más larga es de 971.912. A partir de este valor, se calcula la distancia límite en el valor de 97. A medida que el valor de la distancia límite decrece, el problema es más restricto y la complejidad en su solución aumenta. En las figuras 27 y 28 se presentan los resultados para el caso p05 con y sin la restricción de longitud máxima de ruta. 
Introducción al problema de enrutamiento de vehículos en la logística de distribución

\begin{tabular}{|c|c|c|c|c|c|c|c|}
\hline \multirow[b]{2}{*}{ Caso } & \multicolumn{3}{|c|}{ Yu y $\operatorname{Lin}(2015)$} & \multicolumn{4}{|c|}{ Modelo propuesto } \\
\hline & $\mathrm{BKS}^{a}$ & $\overline{\mathrm{BKS}^{b}}$ & Gap $(\%)$ & CPLEX & $\operatorname{Gap}(\%)^{c}$ & $\operatorname{Gap}(\%)^{d}$ & ${ }^{d} \operatorname{Gap}(\%)^{e}$ \\
\hline 111112 & | 1121.48 & 1322.26 & 31.80 & 1148.04 & 5.29 & 2.37 & -13.18 \\
\hline 111122 & 1061.99 & & & $\begin{array}{l}1069.40 \\
-10.04\end{array}$ & 5.28 & 0.70 & \\
\hline 111212 & 1055.21 & 1322.50 & 51.18 & 1061.00 & 4.11 & 0.55 & -19.77 \\
\hline 111222 & 1085.27 & 7139.32 & 648.42 & 1093.66 & 4.54 & 0.77 & -84.68 \\
\hline 112112 & 897.52 & 969.81 & 26.63 & 899.09 & 2.73 & 0.17 & -7.29 \\
\hline 112122 & 823.19 & & & 817.73 & 2.45 & -0.66 & \\
\hline 112212 & 609.76 & 739.02 & 77.07 & 615.51 & 4.50 & 0.94 & -16.71 \\
\hline 112222 & 575.41 & & & 577.30 & 4.26 & 0.33 & -10.11 \\
\hline 113112 & 916.05 & 1365.40 & 86.16 & 929.68 & 7.39 & 1.49 & -31.91 \\
\hline $\begin{array}{l}113122 \\
11312\end{array}$ & 899.95 & & & 899.39 & 3.33 & -0.06 & \\
\hline 113212 & 689.47 & 1233.18 & 179.19 & 690.01 & 6.75 & 0.08 & -44.05 \\
\hline 113222 & 763.13 & - & - & 764.40 & 3.84 & 0.17 & \\
\hline 131112 & 1417.10 & - & - & 1420.36 & 5.89 & 0.23 & - \\
\hline 131122 & 1376.40 & - & - & 1.356 .36 & 12.00 & -1.46 & - \\
\hline 131212 & 1396.37 & $7-$ & - & 1397.38 & 4.12 & 0.07 & - \\
\hline 131222 & 1371.16 & $6-$ & - & 1407.42 & 15.91 & 2.64 & - \\
\hline 132112 & 1065.52 & $2-$ & - & 1068.32 & 6.00 & 0.26 & - \\
\hline 132122 & 1052.69 & $9-$ & - & 1054.37 & 5.64 & 0.16 & - \\
\hline 132212 & 870.46 & - & - & 882.13 & 7.77 & 1.34 & - \\
\hline 132222 & 761.14 & - & - & 776.11 & 19.71 & 1.97 & - \\
\hline 133112 & 1206.26 & $6-$ & - & 1235.68 & 8.49 & 2.44 & - \\
\hline 133122 & 1068.00 & - & - & 1.067 .16 & 4.56 & -0.08 & - \\
\hline 133212 & 879.52 & - & - & \begin{tabular}{|l|}
891.88 \\
\end{tabular} & 7.36 & 1.41 & - \\
\hline 133222 & 892.52 & - & - & 896.31 & 7.07 & 0.42 & - \\
\hline 121112 & 1686.16 & $5-$ & - & $\begin{array}{l}1697.93 \\
17264\end{array}$ & 13.55 & $\begin{array}{l}0.70 \\
3.33\end{array}$ & - \\
\hline 121122 & 1671.12 & - & - & $\begin{array}{l}1726.84 \\
171090\end{array}$ & $\begin{array}{l}22.52 \\
15.10\end{array}$ & 3.33 & - \\
\hline $\begin{array}{l}121212 \\
121222\end{array}$ & $\begin{array}{l}1650.02 \\
1656.2\end{array}$ & - & $\begin{array}{l}\text { - } \\
\end{array}$ & $\begin{array}{l}17119.95 \\
1857.54\end{array}$ & $\begin{array}{l}15.10 \\
30.41\end{array}$ & $\begin{array}{l}4.24 \\
12.15\end{array}$ & - \\
\hline $\begin{array}{l}122112 \\
12112\end{array}$ & 1457.20 & - & - & $\begin{array}{l}1037.70 \\
\end{array}$ & $\begin{array}{l}30.75 \\
15.71\end{array}$ & 5.52 & $\overline{-}$ \\
\hline 122122 & 1283.15 & $5-$ & - & 1327.12 & 8.82 & 3.43 & - \\
\hline 122212 & 1040.04 & $4-$ & - & 1036.93 & 5.29 & -0.30 & - \\
\hline 122222 & 890.47 & - & - & 887.82 & 3.17 & -0.30 & - \\
\hline 123112 & 1443.40 & - & - & 1473.42 & 15.64 & 2.08 & - \\
\hline 123122 & 1500.55 & $5-$ & - & 1523.08 & 20.44 & 1.50 & - \\
\hline $\begin{array}{l}123212 \\
123222\end{array}$ & 1193.02 & - & - & 1207.31 & 12.87 & 1.20 & - \\
\hline 123222 & 1109.19 & & & 1134.72 & 9.22 & 2.30 & - \\
\hline
\end{tabular}

TABLA NRO. 16. Resultados computacionales para los casos en Tuzun y Burke (1999).

Elaboración propia. 


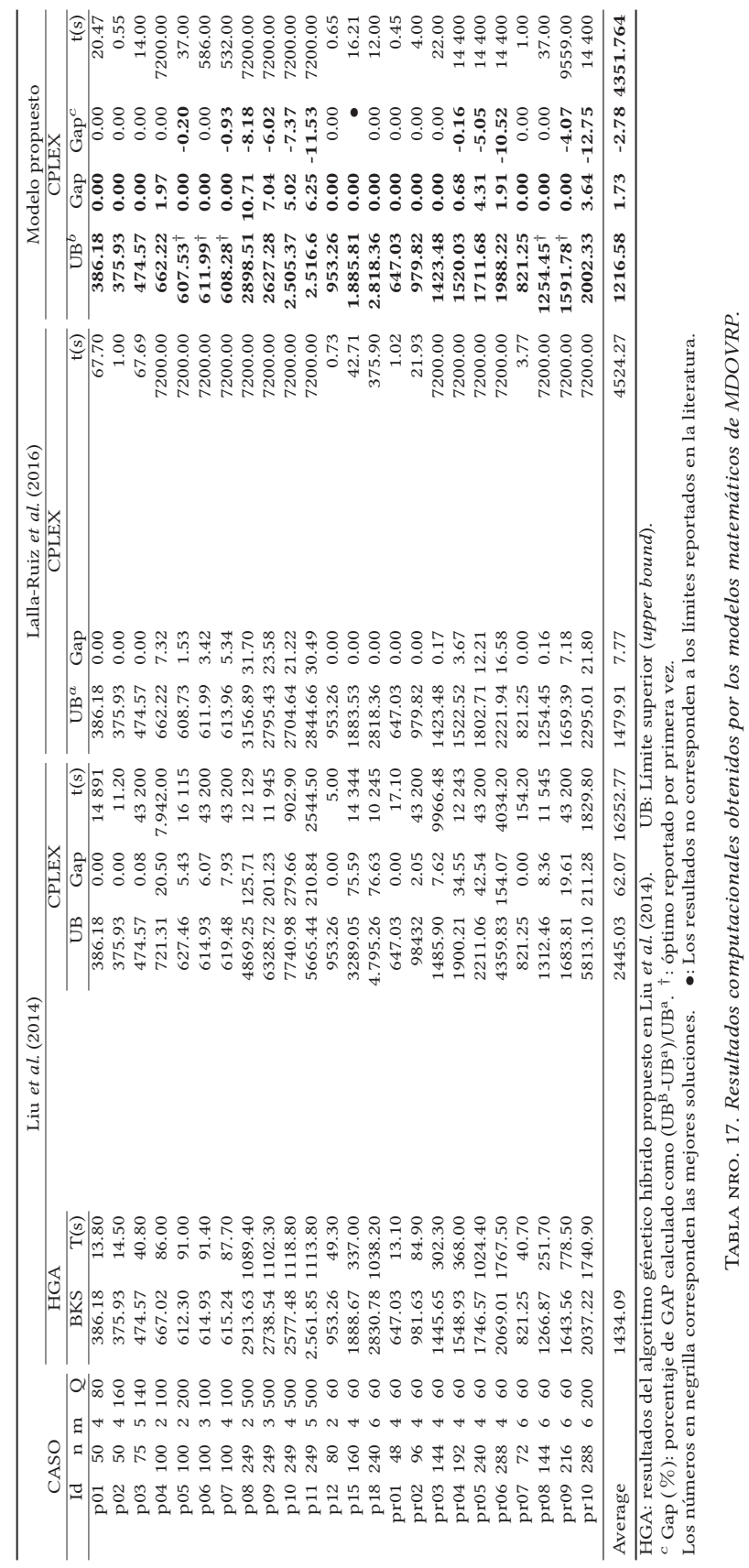




\begin{tabular}{|c|c|c|c|c|c|c|c|c|c|}
\hline \multirow{2}{*}{\multicolumn{3}{|c|}{ Instancia }} & \multirow{2}{*}{\multicolumn{3}{|c|}{$\frac{\text { Modelo sin restricción }}{\text { CPLEX }}$}} & \multicolumn{4}{|c|}{ Modelo con restricción } \\
\hline & & & & & & \multirow[b]{2}{*}{$\mathrm{UB}^{\mathrm{b}}$} & \multicolumn{2}{|c|}{ CPLEX } & \multirow[b]{2}{*}{ Dist. } \\
\hline$I D$ & $n$ & $m Q$ & $\mathrm{UB}^{\mathrm{a}}$ & Gap & $t(s)$ & & Gap & $\mathrm{t}(\mathrm{s})$ & \\
\hline p01 & 50 & 480 & 386.18 & 0.00 & 20.47 & 393.43 & 0.00 & 7 & 48 \\
\hline p02 & 50 & 4160 & 375.93 & 0.00 & & 376.95 & 0.00 & 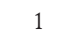 & 69 \\
\hline p03 & 75 & 5140 & 474.57 & & 14.00 & & 0.00 & & 48 \\
\hline p04 & 100 & 2100 & 662.22 & 1.97 & 7200.00 & 664.78 & 2.32 & 36000 & 66 \\
\hline p05 & 100 & 2200 & 607.53 & & 37.00 & 608.37 & 0.00 & & 97 \\
\hline p06 & 100 & 3100 & 611.99 & 0.00 & 586.00 & 613.99 & 0.00 & 130 & 76 \\
\hline p07 & 100 & 4100 & 608.28 & 00 & 32.00 & & 0.92 & 12 & 56 \\
\hline p08 & 249 & 2500 & 2898.51 & 10.71 & 7200.00 & 2868 & 8.92 & 450 & 211 \\
\hline p09 & 249 & 3500 & 2627.28 & .04 & 7200.00 & 2648 & .17 & 45 & 195 \\
\hline p10 & 249 & 4500 & 2505 & 02 & 7200 & 250 & 4.86 & 45 & 155 \\
\hline p11 & 249 & 5500 & 25196.60 & 6.25 & 7200.00 & 2466.28 & 3.72 & 45000 & 146 \\
\hline p12 & 80 & 260 & & & & 957 & 0.00 & 1 & 89 \\
\hline p15 & 160 & 460 & 1885.81 & 0.00 & 16.21 & 1889 & 0.00 & 6 & 100 \\
\hline p18 & 240 & 660 & 2818.36 & & 12.00 & 2826 & 00 & 18 & 100 \\
\hline pr01 & 48 & 4200 & 647.03 & 0.00 & 0.45 & 653.77 & 0.00 & 1 & 100 \\
\hline pr02 & 96 & 4195 & 979.82 & & 4.00 & 986.96 & 0.00 & 16 & 160 \\
\hline pr03 & 144 & 4190 & 1423.48 & 0.00 & 22.00 & 1424.90 & 0.00 & 57 & 227 \\
\hline pr04 & 192 & 4185 & 1520.03 & 0.68 & 14400.00 & & 0.88 & 36000 & 168 \\
\hline pr05 & 240 & 4180 & 1711.68 & 4.31 & 14400.00 & 1713.91 & 4.22 & 4500 & 162 \\
\hline pr06 & 288 & 4175 & 1988.22 & 1.91 & 14400.00 & 1987.12 & 1.67 & 45000 & 154 \\
\hline pr07 & 72 & 6200 & 821.25 & 0.00 & 1.00 & 831.45 & 0.00 & 1 & 110 \\
\hline pr08 & 144 & 6190 & 1254.45 & 0.00 & 37.00 & 1257.49 & 0.00 & 42 & 163 \\
\hline pr09 & 216 & 6180 & 1591.78 & 0.00 & 9559.00 & 1594.01 & 0.00 & 32000 & 146 \\
\hline pr010 & 288 & 6170 & 2002.33 & 3.64 & 14400.00 & 1982.71 & 2.38 & 45000 & 164 \\
\hline $\mathrm{Pr}$ & romed & & 1434.91 & 7.77 & 4524.27 & 1408.24 & 1.50 & 18537.50 & 125.42 \\
\hline
\end{tabular}

TABLA NRO. 18. Resultados con y sin restricción de distancia para el MDOVRP. Elaboración propia.

\subsection{Resultados de versiones estándar del VRP}

Para la validación de la metodología y capacidad del modelo en la solución de las versiones estándar, se implementa una comparación con el modelo MTZ.

Para el CVRP, se utiliza el conjunto de instancias $P$, propuesto por Augerat et al. (1998), donde el número de clientes $N$ se encuentra en el rango de 15 y 100 con capacidades de carga de los vehículos $(Q)$ y valores en el rango de 35 y 3000. El segundo problema estudiado es el MDVRP. Se implementaron instancias entre 50 y 100 clientes de 2 a 6 depósitos. La evaluación del modelo para el CLRP se hace sobre 30 casos de prueba. La cantidad de depósitos esta entre 5 o 10, la cantidad de clientes $N$ es un valor que varía entre 20,50,100,200 y la capacidad $Q$ corresponde a valores entre 70 y 150 según el caso. Se tiene en cuenta que en estudios previos la distancia entre dos nodos se 
afecta por un factor que multiplica por 100 su valor para todas las instancias de estos conjuntos de datos. En primera instancia, es utilizado el modelo matemático con las restricciones MTZ y el modelo matemático propuesto sobre el conjunto de instancias para el CVRP. Los resultados obtenidos se muestran en la Tabla 19. En la Tabla nro. 20, son presentados los resultados obtenidos para las instancias del MDVRP, tanto para el modelo radial, como para el modelo MTZ. En la Tabla nro. 21, se presentan los resultados para las instancias del CLRP para el modelo radial y el MTZ. En los resultados el GAP, es reportado de dos formas:

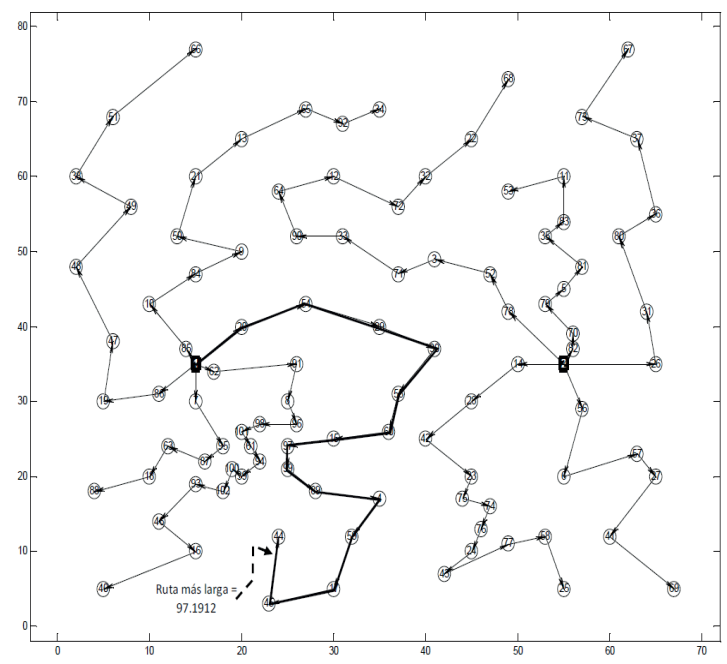

FIGURA NRO. 27. Óptimo sin considerar restricción de longitud máxima de ruta para po5. Elaboración propia.

- La diferencia entre el límite superior y el límite inferior obtenido en CPLEX.

$$
G A P_{B}=\frac{U B-L B}{U B} \times 100 \%
$$

- La diferencia entre la mejor solución conocida, best known solution (BKS), y la mejor solución obtenida en CPLEX.

$$
G A P_{B K S}=\frac{B K S-U B}{B K S} \times 100 \%
$$


Introducción al problema de enrutamiento de vehículos en la logística de distribución

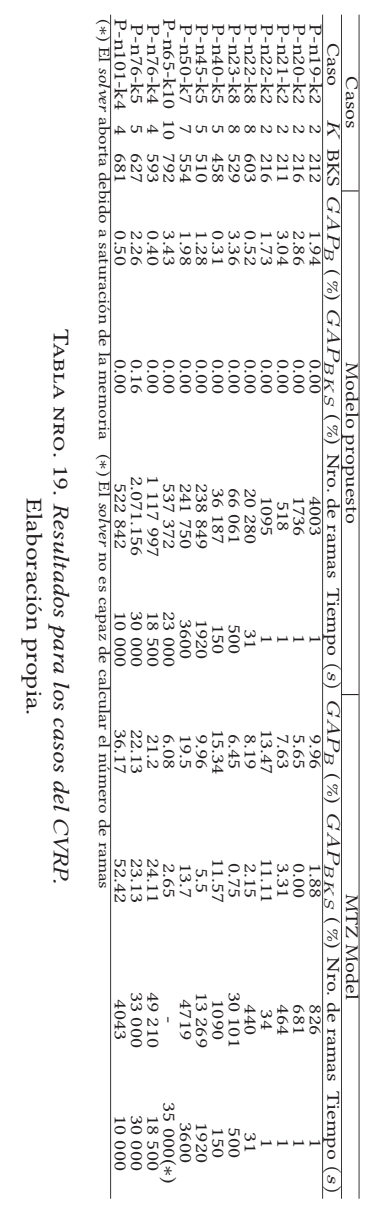


Eliana Mirledy Toro Ocampo, John Fredy Castañeda Londoño, Ramón Alfonso Gallego Rendón

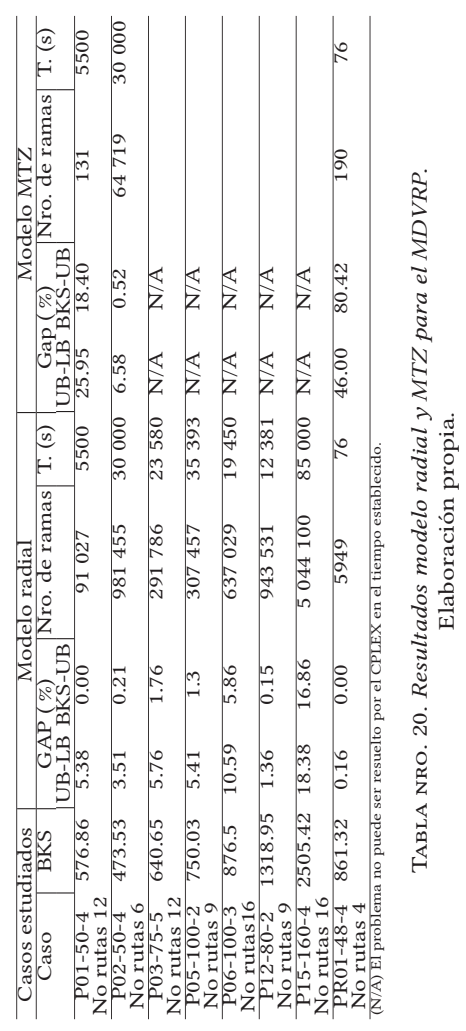


Introducción al problema de enrutamiento de vehículos en la logística de distribución

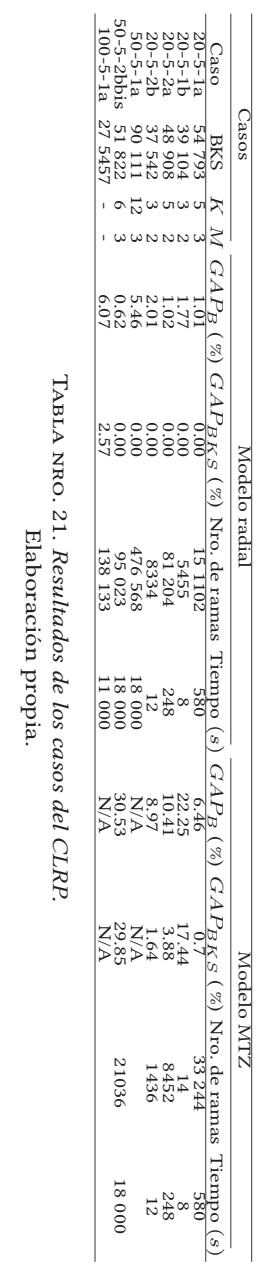




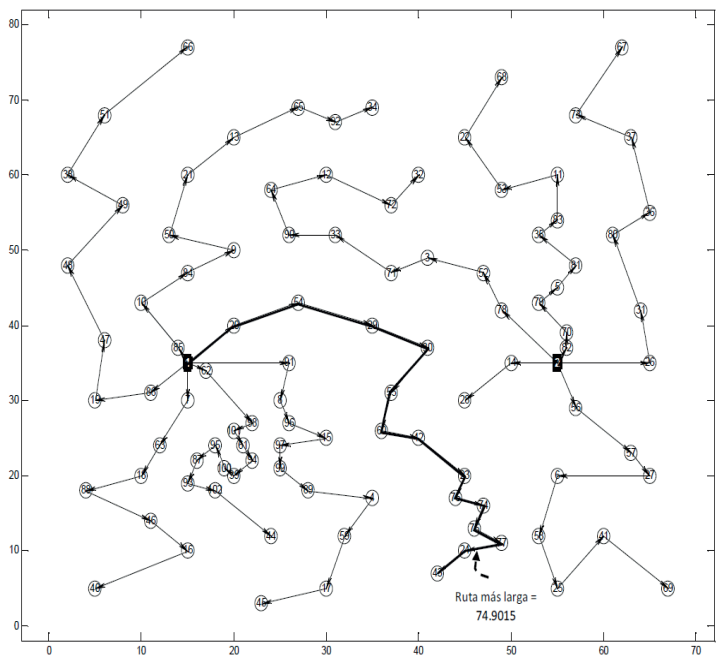

FIGURA NRO. 28. Óptimo considerando restricción de longitud máxima de ruta para po5. Elaboración propia.

Los resultados obtenidos para el CVRP muestran que el modelo radial tiene la capacidad de llegar a los BKS reportados en todos los casos con la excepción de la instancia P-n76-k5, donde se obtiene un GAP del 0.16\%. En el modelo MTZ, alcanzan los BKS solo en instancias pequeñas de 20 clientes o menos. Para las otras instancias, la diferencia con respecto al BKS tiene una variación que va desde $1.88 \%$ al $52.42 \%$. En todos los casos, se define el mismo límite de tiempo de ejecución. Con respecto a la diferencia entre los límites superiores e inferiores, en el modelo radial propuesto, se obtienen unos GAP entre $0.31 \%$ y $3.43 \%$, mientras el modelo MTZ llega a unos GAP entre $5.65 \%$ y $36.17 \%$.

En el MDVRP de las instancias implementadas se infiere que el modelo radial alcanza la solución BKS en algunas de estas instancias, en las otras se evidencia una diferencia respecto al BKS que varía entre el 0.15 y el $1.76 \%$. En referencia al MTZ, este no logra el BKS en ninguna de las instancias analizadas y se obtiene en referencia al BKS una diferencia que varía entre el 0.52 y el $80.42 \%$, en los otros casos no fue posible obtener GAP. La segunda forma de GAP analizado en el problema MDVRP es el UB - LB. En el modelo radial, se alcanzan GAP que varían en el rango entre el 0.16 y $5.76 \%$. En el modelo MTZ, se alcanzan GAP que van desde 6.58 hasta $46.00 \%$, en los otros casos no se obtuvo un GAP.

Para el CLRP, se usaron instancias con 20 y hasta 100 clientes, y 5 depósitos, presentadas en Prins et al. (2006). El modelo propuesto alcanza el BKS en la mayoría de los casos, excepto para la 100-5-1a. Lo anterior, muestra 
Introducción al problema de enrutamiento de vehículos en la logística de distribución

un GAP de $2.57 \%$. El modelo MTZ no logra llegar al BKS en ninguna de las instancias, con un GAP entre $0.7 \%$ y 29.87\%. En la instancia 100-5-1a no se alcanza ningún GAP. Para la diferencia entre los límites superiores e inferiores, el modelo radial logra una variación de GAP entre $0.62 \%$ y $6.07 \%$. Para el MTZ se logra un GAP con variación entre $6.46 \%$ y $30.53 \%$, además no se logra obtener alguna solución.

Se da el mismo tiempo computacional tanto para el modelo radial como para el MTZ en las instancias analizadas. Se pudo inferir que el modelo radial logra un mejor alcance en el árbol de búsqueda en comparación con el modelo MTZ.

En las figuras 29 y 30, se presentan dos de las soluciones óptimas encontradas para el CVRP y CLRP, identificadas como P-n101-k4 y 100-5-1a, respectivamente.

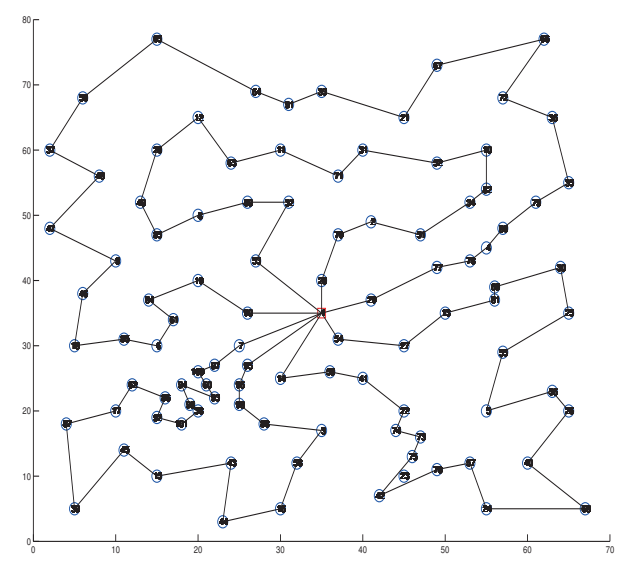

FIgURA NRo. 29. Solución óptima obtenida para el CVRP caso P-n101-k4. Elaboración propia.

\subsection{Resultados problemas CLRP considerando impacto ambiental}

El método $\epsilon$-constraint se usa para generar los frentes de Pareto, usando $\Psi_{2}$ como objetivo y $\Psi_{1}$ como restricción (Fourer et al., 1990). 


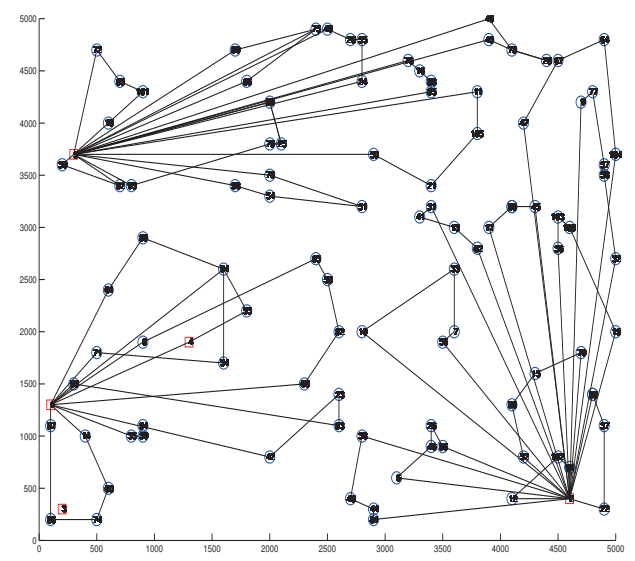

Figura NRO. 30. Solución óptima obtenida para el CLRP del caso 100-5-1a. Elaboración propia.

\subsubsection{Resultados GCLRP}

En este estudio, se utilizaron dos escenarios de prueba, una con 20 y otra con 30 clientes, y ambos con 5 depósitos. La numeración del 1 al 5 corresponde a los depósitos y del 6 en adelante identifica los clientes. Se considera una flota homogénea de vehículos con capacidad de 20 toneladas. El cálculo del consumo del vehículo fue tomado del informe de la Universidad de Michigan, Instituto de Investigación del Transporte (UMITRI, 2014), en el que se estableció que el consumo medio de combustible de un vehículo de estas características es de 1 galón por $15.81 \mathrm{~km}$ recorridos. Este valor se utiliza como referencia para calcular el consumo del vehículo a plena carga, que se estimó en $12 \mathrm{~km}$ por galón.

En cuanto a la cantidad de emisiones por galón de gasolina, se considera $8.70645 \mathrm{~kg}$ de $\mathrm{CO}_{2}$, esta información se relaciona con la guía de consumo de combustible (OEE, 2015). En este informe de emisiones de $\mathrm{CO}_{2}$ varía según el tipo de combustible utilizado y de las características del motor, tales como el tamaño, el tipo, la marca del vehículo y la velocidad óptima de crucero. El costo de las emisiones se calcula con base en los precios cotizados que se presentan en SendeCO2 (2014). El valor de 0.009 USD por kg de $\mathrm{CO}_{2}$. El precio de un galón de gasolina fue consultado en línea ${ }^{1}$. Esta información se establece en función del territorio en el que se contempla el estudio de caso. En nuestro caso, este parámetro fue de 3.92 USD por galón.

${ }^{1}$ http://es.globalpetrolprices.com/gasoline_prices/ 
Introducción al problema de enrutamiento de vehículos en la logística de distribución

Los datos usados para describir el comportamiento del modelo propuesto para el GCLRP se presentan en la Tablas nro. 22 y 23, adicionalmente, se exponen los frentes de Pareto obtenidos en las tablas nro. 24 y 25 para ambos casos de prueba. La Figura nro. 31 muestra la solución del CLRP 20-5 correspondiente al valor mínimo de $\Psi_{1}$, la Figura nro. 32 muestra la solución correspondiente al valor mínimo obtenido para $\Psi_{2}$, y la Figura nro. 33 muestra la solución min- $\max$.

La solución que se presenta en la Figura nro. 31 presenta 1 depósito y 5 rutas. El depósito seleccionado está ubicado en un sitio que sirve como centro de carga desde el cual se atiende la mayor cantidad posible de clientes de acuerdo a su capacidad, manteniendo una relación entre la distancia y la cantidad de carga que se entrega a los clientes. La solución óptima para $\Psi_{1}$ reduce el costo de apertura de los depósitos y el costo de utilización de los vehículos.

\begin{tabular}{|c|c|c|c|c|c|c|}
\hline Param. & $C x$ & $\mathrm{Cy}$ & Param. I & D & Param. Q & 20 \\
\hline 1 & 12 & 39 & 6 & 6 & & \\
\hline 2 & 12 & 24 & 7 & 3 & Param. & W \\
\hline 3 & 38 & 29 & 8 & 9 & 1 & 40 \\
\hline 4 & 32 & 43 & 9 & 5 & 2 & 100 \\
\hline 5 & 37 & 46 & 10 & 7 & 3 & 40 \\
\hline 6 & 4 & 20 & 11 & 3 & 4 & 80 \\
\hline 7 & 22 & 7 & 12 & 3 & 5 & 40 \\
\hline 8 & 20 & 2 & 13 & 2 & & \\
\hline 9 & 21 & 7 & 14 & 2 & Param. & 0 \\
\hline 10 & 4 & 21 & 15 & 2 & 1 & 1346 \\
\hline 11 & 1 & 12 & 16 & 3 & 2 & 1383 \\
\hline 12 & 4 & 2 & 17 & 2 & 3 & 1066 \\
\hline 13 & 22 & 21 & 18 & 7 & 4 & 513 \\
\hline 14 & 14 & 36 & 19 & 5 & 5 & 558 \\
\hline 15 & 16 & 40 & 20 & 3 & & \\
\hline 16 & 12 & 40 & 21 & 8 & Param. F & 100 \\
\hline 17 & 13 & 47 & 22 & 7 & & \\
\hline 18 & 15 & 36 & 23 & 8 & & \\
\hline 19 & 3 & 41 & 24 & 4 & & \\
\hline 20 & 12 & 49 & 25 & 3 & & \\
\hline 21 & 6 & 43 & & & & \\
\hline 22 & 14 & 32 & & & & \\
\hline 23 & 50 & 11 & & & & \\
\hline 24 & 29 & 18 & & & & \\
\hline 25 & 37 & 36 & & & & \\
\hline
\end{tabular}

TABLA NRO. 22. GCLRP 205.

Elaboración propia. 
Introducción al problema de enrutamiento de vehículos en la logística de distribución

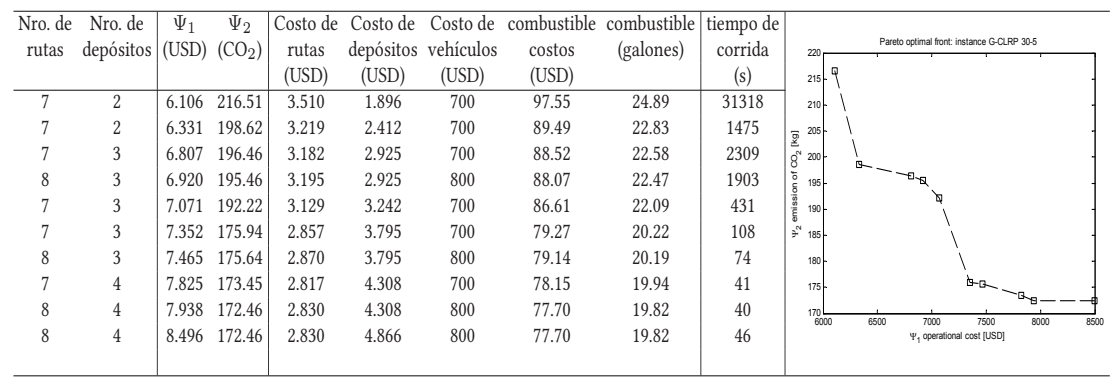

TABLA NRO. 25. Resultados computacionales G-CLRP 30-5.

Elaboración propia.

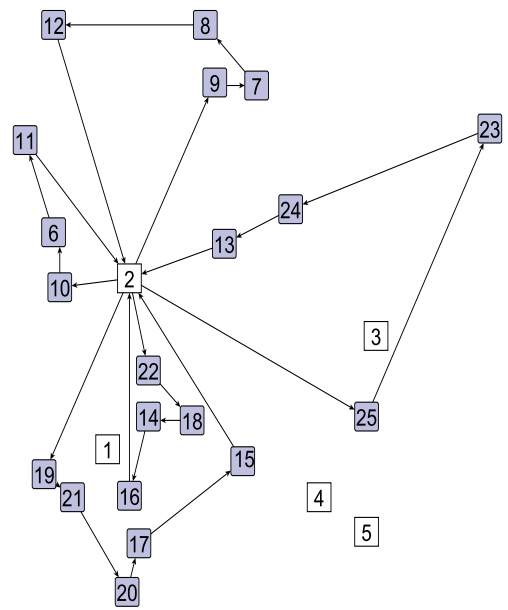

FIgURA NRO. 31. G-CLRP 20-5. Solución costos operacionales $\Psi_{1}=4764$ USD; $\Psi_{2}=180.6 \mathrm{~kg} \mathrm{CO}$.

Elaboración propia. 

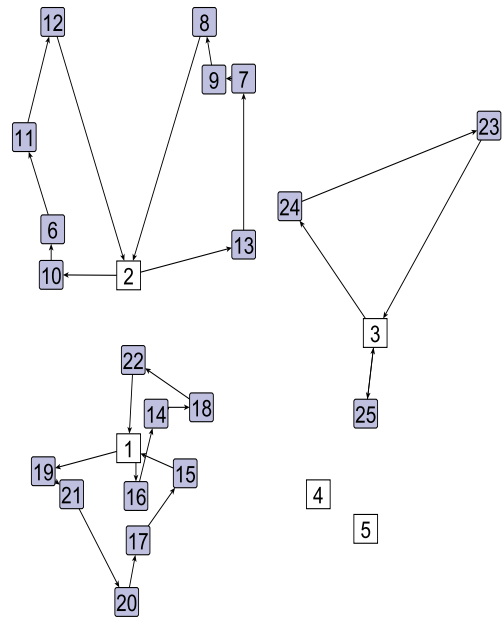

Figura NRo. 32. G-CLRP 20-5. Solución considerando emisiones $\Psi_{1}=7790$ USD, $\Psi_{2}=142.25 \mathrm{~kg} \mathrm{CO}$ Elaboración propia.

La solución presentada en la Figura nro. 32 corresponde a la minimización de $\Psi_{2}$ (aspecto ambiental). En este escenario, 3 depósitos son seleccionados y se identifican 6 rutas. Se puede apreciar que las distancias viajadas por los vehículos son más cortas comparadas con el escenario anterior. Cuando se atienden los clientes, la prioridad de entregas está relacionada con los clientes de mayor demanda y/o con la identificación del cliente más cercano, los clientes de mayor demanda se atienden lo más rápido posible, esto disminuye el consumo de combustible y, por lo tanto, se minimiza la cantidad de emisiones emitidas. Cuando se considera esta función objetivo, la dirección de la ruta toma relevancia debido a que impacta directamente las emisiones.

En el tercer escenario, la Figura nro. 33, la solución min-max combina los costos operacionales y el impacto ambiental. Bajo estas nuevas condiciones, 2 depósitos son abiertos y 5 rutas son seleccionadas. Hay un claro balance entre los costos operativos y el consumo de combustible. Cuando se considera la reducción de emisiones, da prioridad a las entregas de mayor tamaño lo más pronto posible, identifica rutas cortas y tiene en cuenta la dirección del vehículo. Los costos operacionales influyen en el resultado, en términos de número de depósitos y número de rutas usadas.

La Figura nro. 34 muestra la solución pra el CLRP 30-5 cuando se minimiza el valor de $\Psi_{1}$, la Figura nro. 35 muestra la solución correspondiente al valor mínimo para $\Psi_{2}$ y la Figura nro. 36 muestra la solución min-max. La solución de la Figura nro. 34 corresponde a la respuesta óptima para el G-CLRP 30-5 considerando los costos operativos $\left(\Psi_{1}\right)$. Esta solución presenta 2 depósitos y 7 rutas. Como en el caso anterior, la solución óptima atiende los clientes utilizando la menor cantidad de depósitos y vehículos. 


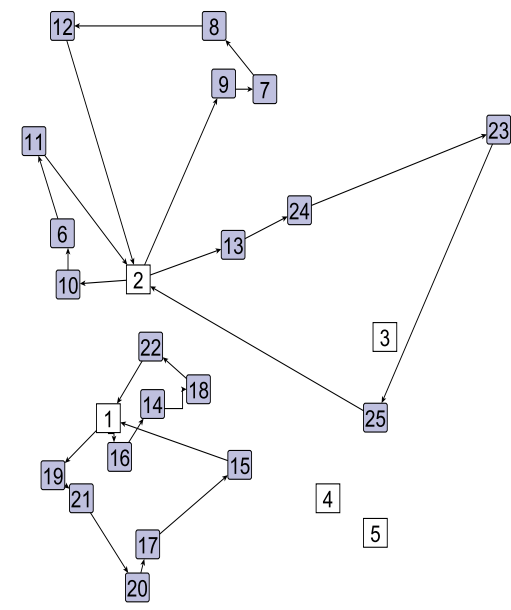

FIGURA NRO. 33. G-CLRP 20-5. Solución min-max $\Psi_{1}=5727$ USD; $\Psi_{2}=153.45 \mathrm{~kg} \mathrm{CO}$ Elaboración propia.

En la Figura nro. 35, la solución que minimiza las emisiones $\left(\Psi_{2}\right)$ presenta 4 depósitos y 8 rutas. Las rutas son cortas y dan prioridad a los clientes con mayor demanda con el fin de reducir el peso del vehículo cargado, lo que influye en la cantidad de combustible requerido por los vehículos.

En la Figura nro. 36 se presenta la solución min-max para el G-CLRP 30-5 con 3 depósitos y 7 rutas. Igual que en el caso previo, se observa una compensación entre los costos operacionales y el impacto ambiental. Esta solución trata de balancear el número de depósitos usados y la cantidad de combustible consumido.

Además, se utilizaron otros 6 casos propuestos por Prins et al. (2007) para realizar un análisis de sensibilidad que permita verificar la eficacia del modelo y corroborar la observación realizada con los dos casos previos en el contexto del CLRP, se aumentó el número de vehículos dado que se observaron el impacto del consumo de combustible y las emisiones son reducidas.

Los resultados de los 5 primeros casos mostrados en la Tabla nro. 26 evidencian frentes óptimos de Pareto en los cuales la solución obtenida, minimizando $\Psi_{1}$, corresponde a aquellas presentadas como BKS en Escobar et al. (2014). Al minimizar $\Psi_{1}$, se observa la necesidad de un menor número de depósitos y vehículos, y mayores longitudes promedio de los arcos utilizados en la solución óptima respecto a lo que se obtiene al minimizar $\Psi_{2}$. Esto se traduce en que un compromiso óptimo entre costos operativos y total de emisiones privilegia realizar rutas largas con pocos vehículos y depósitos desde el punto de vista del costo operativo. Sin embargo, desde el punto de vista de la polución (minimizando $\Psi_{2}$ ), se privilegia la utilización de más vehículos realizando rutas más cortas. Esta observación podría 
ser diferente si algo como el consumo de combustible para el arranque de cada vehículo fuera considerado.

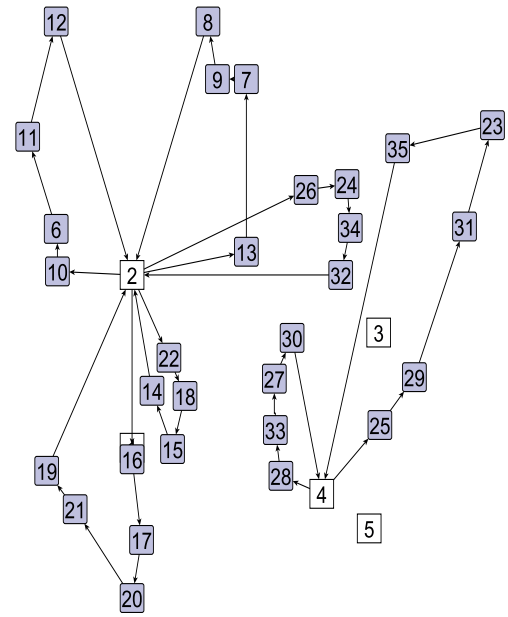

Figura NRo. 34. G-CLRP 30-5. Solución costos operativos $\Psi_{1}=6106 \mathrm{USD} ; \Psi_{2}=216.5 \mathrm{~kg} \mathrm{CO}$. Elaboración propia.

El último caso de la Tabla nro. 26 corresponde a la instancia 100_10_1; la cual es de difícil solución a través de programación matemática exacta. El modelo propuesto es escalable eficientemente hasta casos del orden de 50 ciudades y 5 depósitos. En este caso de 100 ciudades y 10 depósitos, se obtienen soluciones con un GAP inferior al $4 \%$. Sin embargo, estos resultados permiten obtener una adecuada sensibilidad sobre el comportamiento del frente en casos de mayor tamaño, en donde las observaciones anteriores siguen siendo válidas.

Es interesante notar que las soluciones obtenidas relajando $\Psi_{1}$ corresponden a minimizar el PollutionRouting Problem (PRP) considerando los parámetros presentados al inicio de esta sección. Por lo tanto, estos resultados pueden ser considerados como referencia para fines comparativos del problema monobjetivo de PRP.

Los resultados también permiten evidenciar que cuando se aplica el criterio min-max para la selección de una solución del frente, el compromiso entre el costo de inversión y el impacto ambiental corresponden a una solución intermedia del frente.

\subsubsection{Resultados GOLRP}

En todas los casos analizados, se observa la conformación de un frente de Pareto, en donde las soluciones presentan diferentes características, relacionadas con el compromiso que se requiere entre emisiones y costos de la operación. A manera de ejemplo, en la Figura nro. 37 se presentan 3 puntos representativos del frente óptimo de Pareto, obtenido 


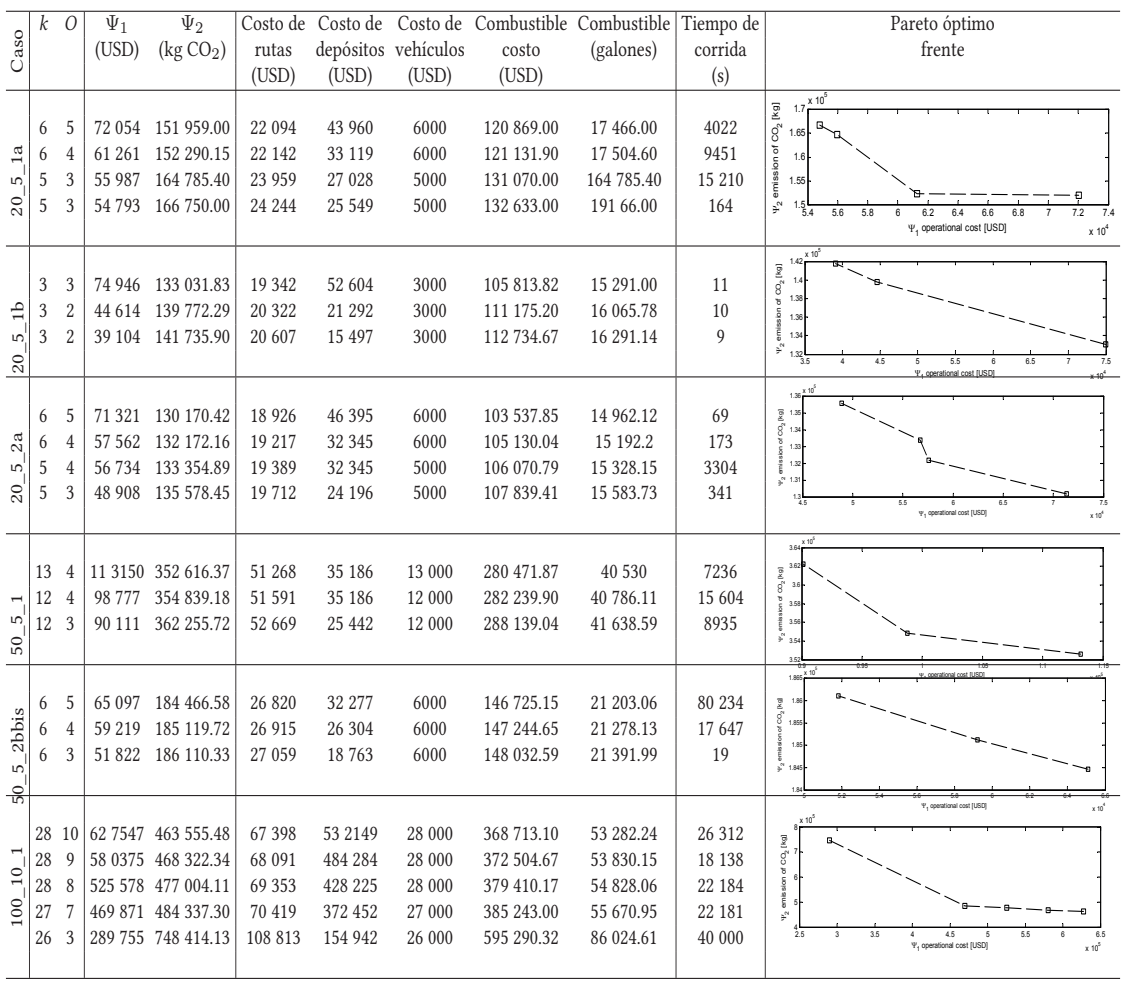

TABLA NRO. 26. Resultados computacionales para los casos tomados de Prins et al. (2007).

Elaboración propia.

para el caso 100_10_2b. El punto A corresponde a la minimización de $\Psi_{1}$ (costos operativos), el punto C corresponde a la minimización del valor de $\Psi_{2}$ (impacto ambiental) y el punto B corresponde al criterio min-max, que usualmente corresponde a un valor intermedio del frente de Pareto.

En la Figura nro. 37, se observa que, para el punto A, la solución es conformada por un número mínimo de depósitos y de vehículos por lo cual, la longitud promedio de las rutas que conectan los clientes con los depósitos es mayor que la obtenida considerando emisiones. Esto implica que los vehículos hacen un mayor recorrido lo cual, a su vez, genera un mayor consumo de combustible y consecuentemente un mayor número de emisiones. Adicionalmente, a medida que se usan menos vehículos, la cantidad de carga transportada por vehículo es mayor, lo cual afecta en forma directa el consumo de combustible, tal como se observa de la función objetivo $\Psi_{2}$.

El punto C del frente muestra la minimización de la función $\Psi_{2}$ (impacto ambiental). Se observa que la solución se conforma de un mayor número de depósitos y rutas que el caso anterior. Así, las rutas que conectan 
los clientes con los depósitos también son de menor lóngitud promedio, lo cual implica menores recorridos de los vehículos y, por lo tanto, una entrega rápida de la carga. Consecuentemente, los vehículos presentan un menor consumo de combustible y un menor número de emisiones.

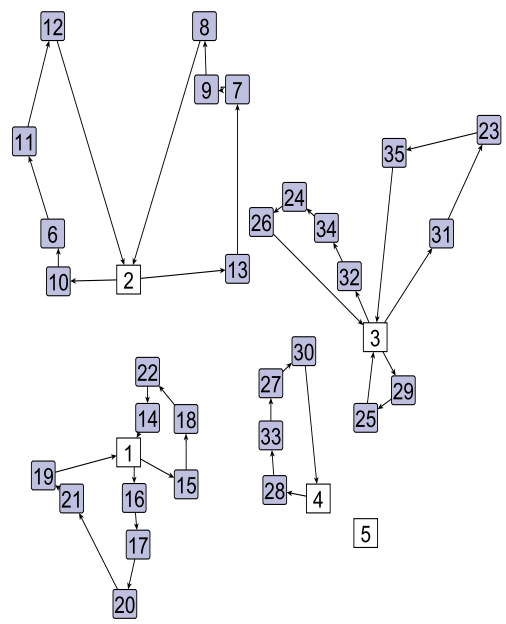

FigURA NRO. 35. G-CLRP 30-5. Solución considerando emisiones $\Psi_{1}=8496$ USD, $\Psi_{2}=172.46 \mathrm{~kg} \mathrm{CO}$. Elaboración propia.

Es intuitivo considerar que se va a consumir más combustible, si se usan más vehículos. Sin embargo, en el contexto de CLRP, cuando se considera la minimización del consumo de combustible, por lo general, el uso de más vehículos no implica alargar la distancia de recorrido. Es interesante notar que al aumentar el número de vehículos, el consumo de combustible y la emisión total se pueden reducir. El uso de algunos vehículos a plena capacidad no implica necesariamene el ahorro de combustible; mientras que el uso de más vehículos, sin ser cargadas a su plena capacidad, se puede traducir en una reducción de costos de combustible y un menor impacto ambiental. A largo plazo, al tener más depósitos y más rutas, la economía de combustible puede compensar los costos iniciales. Así, considerar el efecto de la cantidad de carga transportada impacta de forma directa el número de emisiones ya que usar más depósitos implica una mayor cercanía con los clientes.

El punto intermedio B del frente es seleccionado con base en el criterio min-max. Se observa un valor intermedio en el número de depósitos, vehículos y longitud promedio de las rutas. Similarmente a los casos anteriores, se observa cómo el número de depósitos afecta inversamente las longitudes de las rutas que conectan clientes y depósitos. Esto se traduce en que un compromiso óptimo entre costos operativos y total de emisiones privilegia realizar rutas largas con pocos vehículos y depósitos desde el punto de vista del costo operativo. Sin embargo, desde 
el punto de vista de la polución (minimizando $\Psi_{2}$ ), se privilegia la utilización de más vehículos realizando rutas más cortas. Esta observación podría ser diferente si se consideran aspectos como el consumo de combustible al encender el vehículo antes de alcanzar la velocidad de crucero. Estas observaciones son consistentes para todos los escenarios utilizados en las pruebas del modelo.

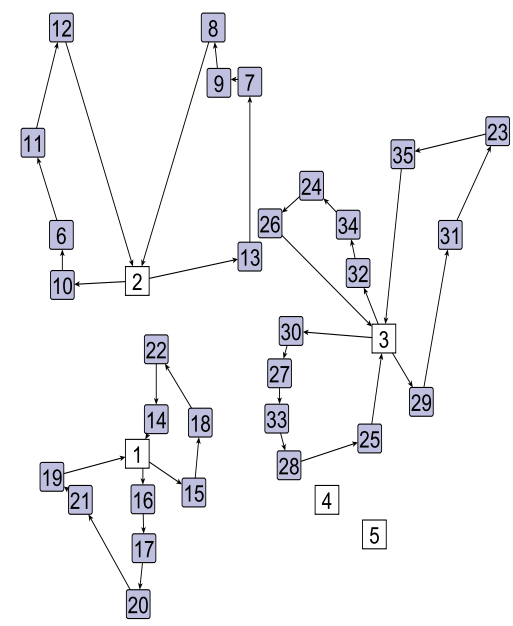

FiguRA NRO. 36. G-CLRP 30-5. Solución min max $\Psi_{1}=7352$ USD; $\Psi_{2}=175.94 \mathrm{~kg} \mathrm{CO}$. Elaboración propia.

En la Tabla nro. 27 se presentan los resultados obtenidos al aplicar el modelo propuesto a los 16 casos de prueba, tomados de Prins et al. (2007). Las columnas 2-5 presentan información sobre la solución óptima obtenida en cada punto del frente y correspondiente al número de rutas, el número de depósitos, el valor de la función objetivo $\Psi_{1}$ y el valor de la función objetivo $\Psi_{2}$, respectivamente. Las columnas 6-8 presentan la discriminación de los tres términos que componen las funciones objetivo de costos operativos $\Psi_{1}$, las cuales corresponden al costo total de viajar a través de las rutas radiales, usadas para entregar la mercancía a los clientes; la suma de los costos de apertura de los depósitos y los costos fijos asociados con el uso de los vehículos, respectivamente. Las columnas 9 y 10 presentan la discriminación de los dos términos que componen el segundo objetivo $\Psi_{2}$, los cuales corresponden al consumo de combustible y al total de emisiones asociadas al mismo. La columna 11 reporta el tiempo computacional utilizado y la columna 12 muestra el frente óptimo de Pareto, obtenido para cada instancia. Esta última columna permite concluir que el modelo propuesto tiene la capacidad de generar un conjunto de soluciones que balancea los objetivos propuestos. 


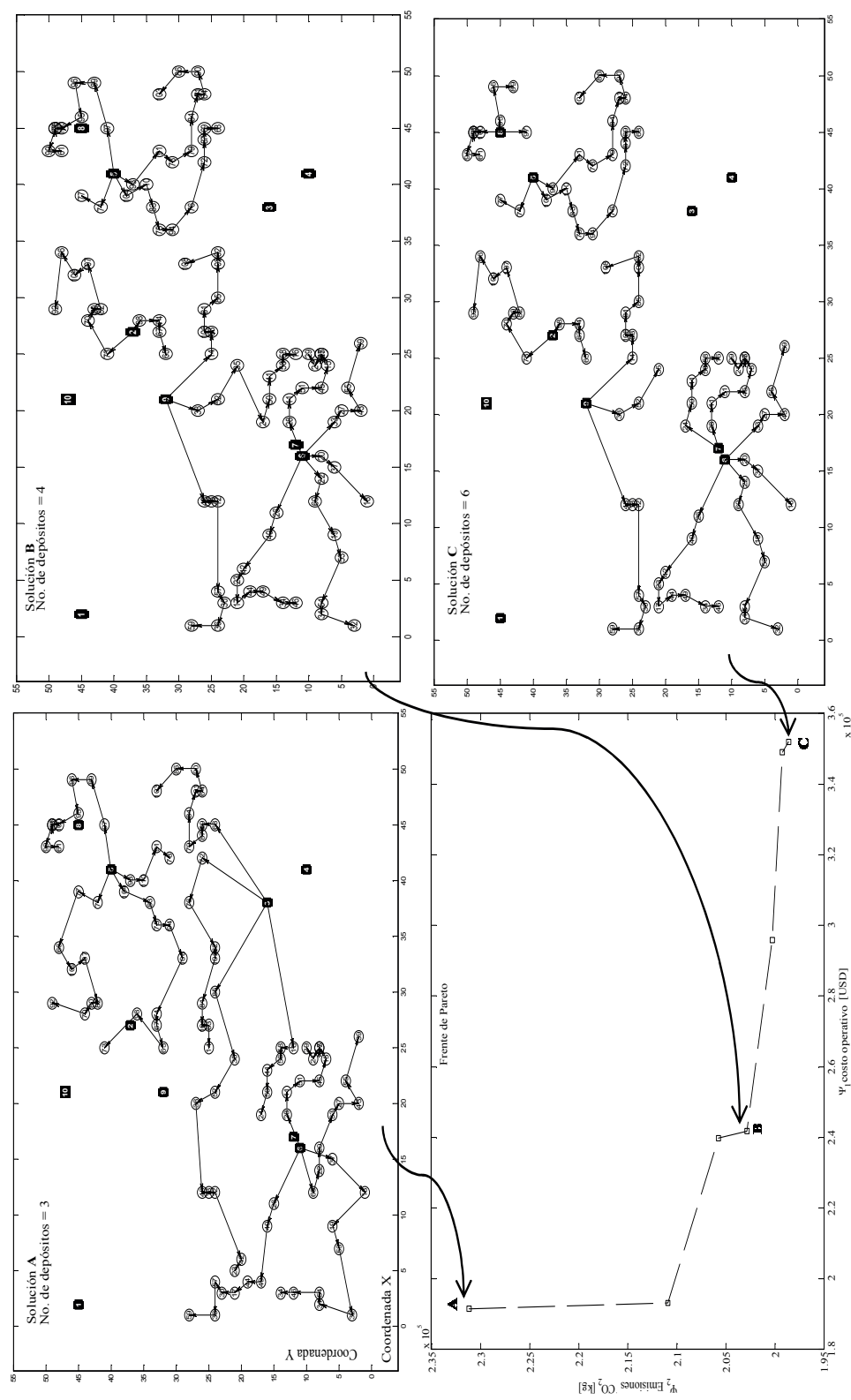

FIGURA NRO. 37. Tres puntos del frente (costos, emisiones y min-max). Elaboración propia. 
Introducción al problema de enrutamiento de vehículos en la logística de distribución

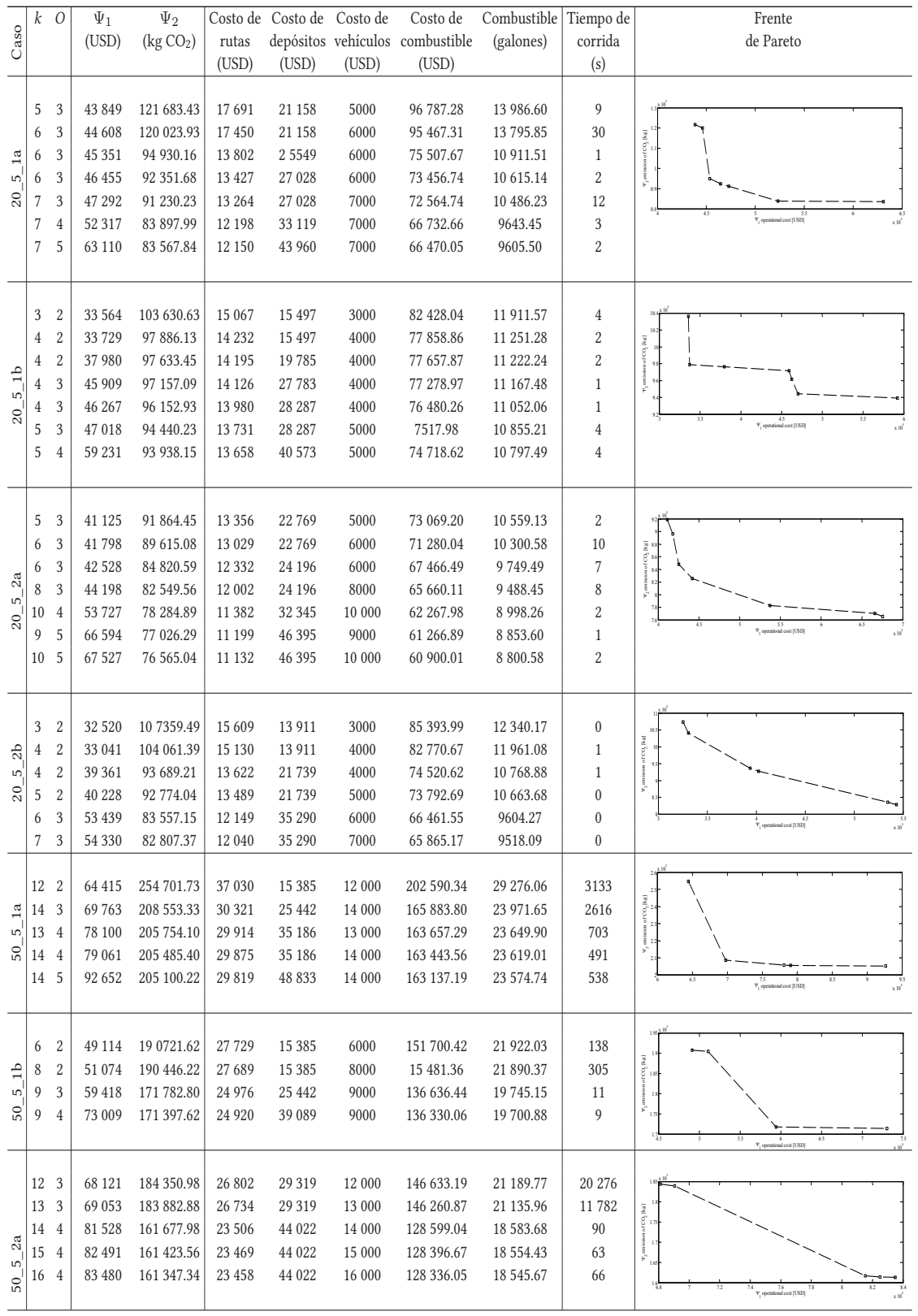




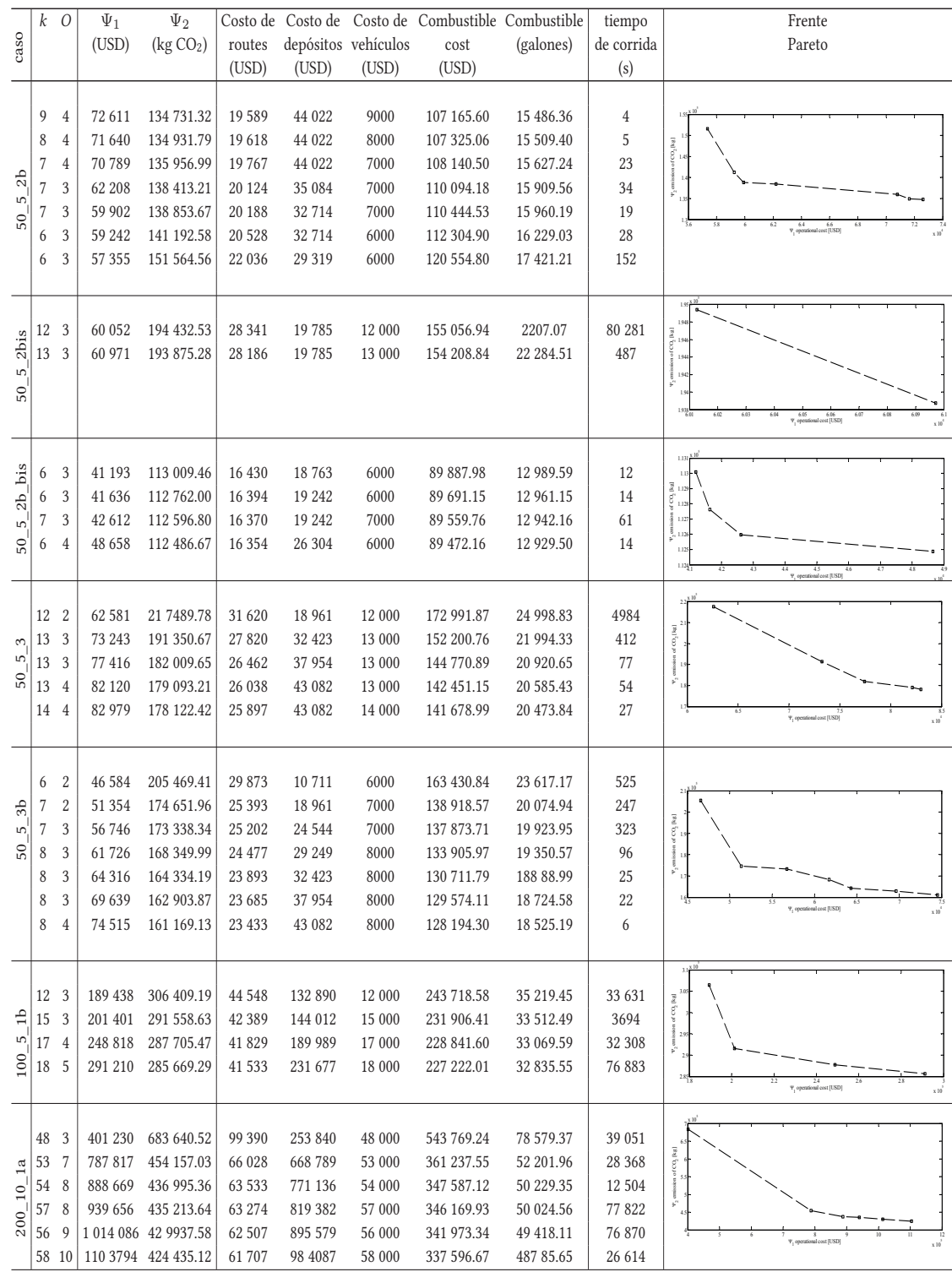




\begin{tabular}{|c|c|c|c|c|c|c|c|c|c|c|c|}
\hline $\begin{array}{l}\text { मे } \\
\text { yु }\end{array}$ & $k$ & 0 & $\begin{array}{c}\Psi_{1} \\
\text { (USD) }\end{array}$ & $\begin{array}{c}\Psi_{2} \\
(\mathrm{~kg} \mathrm{CO})\end{array}$ & $\begin{array}{l}\text { Costo de } \\
\text { routes } \\
\text { (USD) }\end{array}$ & $\begin{array}{c}\text { Costo de } \\
\text { depósitos } \\
\text { (USD) }\end{array}$ & $\begin{array}{l}\text { Costo de } \\
\text { vehículos } \\
\text { (USD) }\end{array}$ & $\begin{array}{c}\text { Combustible } \\
\text { cost } \\
\text { (USD) }\end{array}$ & $\begin{array}{c}\text { Combustible } \\
\text { (galones) }\end{array}$ & $\begin{array}{c}\text { tiempo } \\
\text { de corrida } \\
\text { (s) }\end{array}$ & $\begin{array}{l}\text { Frente } \\
\text { Pareto }\end{array}$ \\
\hline \multirow{4}{*}{$\begin{array}{l}\hat{N}_{1} \\
0^{\prime} \\
\overbrace{}^{\prime} \\
\overbrace{}^{\prime} \\
\text { N }\end{array}$} & 23 & 3 & 350257 & 322497.10 & 46887 & 280370 & 23000 & 256514.93 & 37068.63 & 71118 & \multirow{4}{*}{ 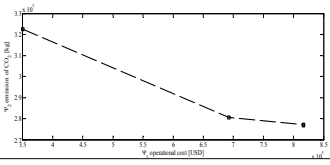 } \\
\hline & 27 & 6 & 692466 & 280542.94 & 40788 & 624678 & 27000 & 223144.50 & 32246.31 & 53679 & \\
\hline & 27 & 7 & 816443 & 277199.73 & 40302 & 749141 & 27000 & 220485.30 & 31862.04 & 30562 & \\
\hline & 27 & 7 & 816389 & 276828.46 & 40248 & 749141 & 27000 & 220189.99 & 31819.36 & 33143 & \\
\hline \multirow{8}{*}{ 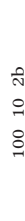 } & 12 & 3 & 191565 & 231167.38 & 33609 & 145956 & 12000 & 183871.07 & 26570.96 & 82745 & \multirow{8}{*}{ 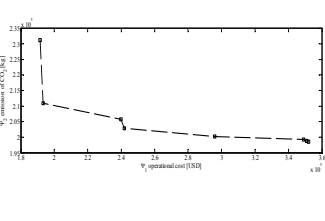 } \\
\hline & 13 & 3 & 193245 & 210875.65 & 30659 & 149586 & 13000 & 167730.97 & 24238.58 & 72779 & \\
\hline & 14 & 4 & 239820 & 205749.88 & 29914 & 195906 & 14000 & 163653.93 & 23649.41 & 44238 & \\
\hline & 14 & 4 & 241796 & 202826.54 & 29489 & 198307 & 14000 & 161328.70 & 23313.40 & 1965 & \\
\hline & 16 & 5 & 295934 & 200225.53 & 29111 & 250823 & 16000 & 159259.85 & 23014.43 & 1378 & \\
\hline & 14 & 6 & 349058 & 199215.84 & 28964 & 306094 & 14000 & 158456.74 & 22898.37 & 4528 & \\
\hline & 16 & 6 & 350987 & 198726.46 & 28893 & 306094 & 16000 & 158067.48 & 22842.12 & 1510 & \\
\hline & 17 & 6 & 351966 & 198580.45 & 28872 & 306094 & 17000 & 157951.35 & 22825.34 & 1279 & \\
\hline
\end{tabular}

TABla nRo. 27. Resultados computacionales del GOLRP usando Prins et al. (2007).

Elaboración propia.

De los resultados, se observa que, en los casos de hasta 20 clientes, se obtienen soluciones con un GAP igual a $0 \%$; en los casos de 50 clientes, la mayoría de las soluciones obtenidas presentan una GAP de $0 \%$ y unas pocas un GAP inferior al $3 \%$ en los casos estudiados con 100 clientes, se obtienen soluciones con un GAP inferior al $2 \%$; y para los de 200 clientes, se obtienen soluciones con un GAP inferior al $3 \%$.

Se observa una solución con 2 puntos en el frente, con costos operativos similares, lo que implica que entre estas soluciones no podrá existir ninguna otra que implique diferente número de vehículos o combinaciones de los depósitos. Lo anterior indica una disposición geográfica de los depósitos que resultan ser adecuados centros de carga.

Se ha observado en los experimentos que, cuando se utiliza la minimización de emisiones $\left(\psi_{2}\right)$ y la función objetivo y los costos operativos $\left(\psi_{1}\right)$ como restricción; el modelo se resuelve mucho más rápido que cuando se realiza a la inversa. Esto permite la solución de los casos más grandes. Generalmente, cuando se minimizan emisiones, el número de depósitos seleccionados es mayor y, por lo tanto, la complejidad matemática del problema es menor; esto se refleja en el tiempo computacional necesario para su solución.

Es interesante notar que las soluciones obtenidas relajando $\Psi_{1}$ corresponden a minimizar el PollutionRouting Problem (PRP) considerando los parámetros presentados al inicio de esta sección. Por lo tanto, estos resultados pueden ser considerados como referencia para fines comparativos del problema monobjetivo de PRP. 


\subsection{Análisis de la metodología aproximada para el GVRPPCWE}

El GVRPPCWE se encuentra dentro del grupo de problemas denominados NP-Duros, que combina dos tipos de problemas de enrutamiento: el problema de enrutamiento con restricción de capacidad (CVRP) y el problema de enrutamiento abierto (OVRP). Debido a esto, el algoritmo descrito en la sección 5.2 está diseñado de forma tal que se ejecute de manera eficiente, sin repetir operaciones de cómputo, y los movimientos de búsqueda van en la dirección de las soluciones óptimas.

\subsubsection{Parámetros de implementación del algoritmo}

Los parámetros del algoritmo son importantes ya que de ellos depende el desempeño del mismo. Se tomaron como referencia las formulaciones propuestas por Penna et al. (2013). Los parámetros usados son descritos en 6.2, 6.3, 6.4 y 6.5:

$$
\begin{gathered}
\text { MaxIterLocal }=\# \text { Nodos }+\beta * V \\
\beta=\operatorname{Random}(20)
\end{gathered}
$$

$$
D F=4
$$

$$
\text { MaxIterILS }=(100 * D F)
$$

Donde,

MaxIterLocal: Define el número de iteraciones locales.

$\beta$ : Establece la relación entre la cantidad de iteraciones locales y el número de vehículos del problema. Para cada iteración, este valor será seleccionado aleatorio dentro del dominio $[1,20]$.

$V$ : Cantidad de vehículos de la flota propia y subcontratada.

$D F$ : Factor de profundidad de la búsqueda local definido en el apartado 5.2. Para frentes de Pareto densos, es definido en $D F=4$ pero, para instancias de mayor complejidad computacional (más de 40 clientes), se define en $D F=2$ con el fin de asegurar convergencia en tiempos razonables.

MaxIterILS : Número máximo de iteraciones para el ILS. 


\subsubsection{Verificación de estabilidad y rendimiento}

Para la verificación de la estabilidad del algoritmo con respecto a las soluciones, es utilizado el teorema del límite central. En el proceso, son realizadas $n \geq 30$ ejecuciones para la instancia A-n39-k6 y se obtienen soluciones con bajo coeficiente de variación, lo que permite confirmar que el algoritmo es estable y descendente. En la Tabla nro. 28, se observan los valores obtenidos para las ejecuciones. Los valores de sesgo estandarizado y kurtosis estandarizada se encuentran en un intervalo entre [-2,2], indicando que los datos asociados a las métricas de Pareto no tienen una desviación significativa a la normalidad. En la Figura nro. 38, se muestra una gráfica boxplot de los resultados de las métricas normalizadas con un dato atípico para el tiempo.

\begin{tabular}{ccccc} 
& \multicolumn{4}{c}{ Tiempo Hipervolumen Spacing Spread } \\
\hline Coeficiente de variación & $7.04 \%$ & $2.01 \%$ & $32.46 \%$ & $7.43 \%$ \\
Sesgo estandarizado & 0.700 & -0.076 & 1.542 & -0.418 \\
Kurtosis estandarizada & 0.144 & -0.909 & 0.324 & -0.789 \\
\hline
\end{tabular}

TABLA NRO. 28. Resumen estadístico de 30 ejecuciones. Elaboración propia.

\subsubsection{Resultados obtenidos}

Fueron usadas instancias de la literatura especializada para el problema clásico del CVRP -descritas en Uchoa et al. (2017)-, clasificadas como de complejidad $O\left(v^{n-1} / 2\right)$ para cada objetivo, donde $v$ denota el número de vehículos, $n$ el número de clientes considerando el problema simétrico. En las instancias, el valor de $k$ corresponde al número de vehículos de la flota propia y se calcula como $k=0.8 q / Q$, donde $q$ es el valor de la demanda total de los clientes y $Q$ es la capacidad de los vehículos. La flota de vehículos es homogénea y las rutas subcontratadas no tienen restricción en su tope máximo de vehículos.

Para la comparación de los frentes de Pareto, encontrados en cada ejecución, es utilizada la métrica de hipervolumen que usa valores normalizados y clasifica las soluciones de mejor a peor por su mayor valor, ya que este es más grande a medida que el Pareto se aleja del punto Nadir de referencia. El uso de varias métricas para clasificar las soluciones por su calidad podría resultar no adecuado debido a que las métricas se definen sobre diferentes conceptos geométricos los cuales implican que en algunos casos dichas métricas no coincidan en clasificación si la referencia es un punto y no un Pareto. Para el caso del espaciamiento, esta métrica es usada únicamente para calificar la densidad del frente más no su calidad. En la Tabla nro. 29, se presentan los resultados obtenidos para instancias definidas, 


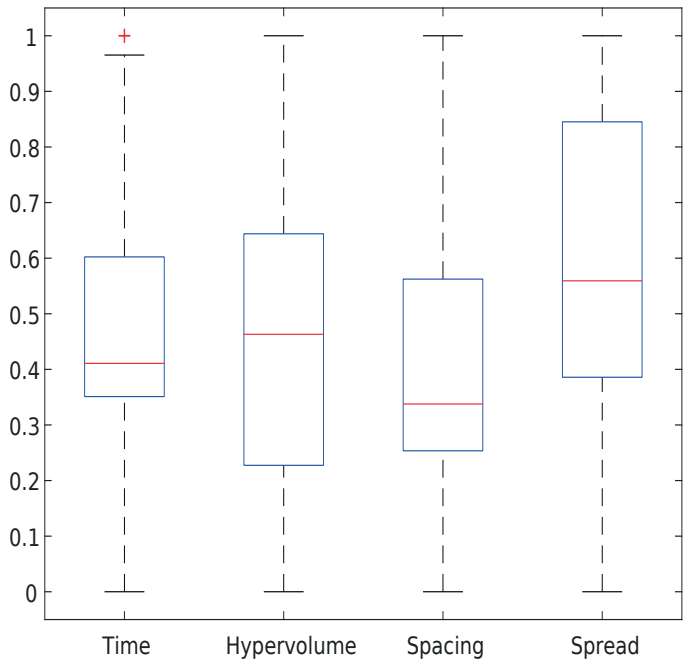

Figura NRO. 38. Boxplot métricas de pareto.

Elaboración propia.

como de baja complejidad matemática, con 50 clientes y hasta 10 vehículos. Para estas instancias, se realizaron 10 ejecuciones de cada una y se seleccionó la mejor solución.

\begin{tabular}{cccccc} 
Instancia & Hipervolumen & Spacing & Spread & Referencia (Nadir) & Tiempo (s) \\
\hline P-n16-k8 & 0.0936 & 1431.111 & 1.070 & $768,3.198 \mathrm{E}+05,148$ & 627 \\
P-n19-k2 & 0.2586 & 1093.707 & 0.979 & $668,2.737 \mathrm{E}+05,159$ & 404 \\
P-n20-k2 & 0.2107 & 1094.128 & 0.762 & $583,2.421 \mathrm{E}+05,102$ & 827 \\
P-n21-k2 & 0.2415 & 1458.129 & 0.815 & $572,2.510 \mathrm{E}+05,94$ & 192 \\
P-n22-k2 & 0.2406 & 1973.739 & 0.999 & $634,2.634 \mathrm{E}+05,59$ & 140 \\
P-n23-k8 & 0.0938 & 1320.923 & 1,035 & $844,4.319 \mathrm{E}+05,120$ & 373 \\
A-n32-k5 & 0.2141 & 3878.744 & 0.821 & $1850,1.169 \mathrm{E}+06,241$ & 1534 \\
A-n33-k5 & 0.2956 & 16817.695 & 1.174 & $2062,1.223 \mathrm{E}+06,245$ & 1738 \\
A-n39-k6 & 0.2212 & 9996.053 & 0.940 & $2434,1.415 \mathrm{E}+06,406$ & 994 \\
P-n40-k5 & 0.2074 & 7688.527 & 0.964 & $1126,7.923 \mathrm{E}+05,110$ & 2028 \\
P-n45-k5 & 0.2342 & 4197.277 & 0.946 & $1475,9.863 \mathrm{E}+05,177$ & 4385
\end{tabular}


Introducción al problema de enrutamiento de vehículos en la logística de distribución

\begin{tabular}{llllll} 
P-n50-k7 & 0.1972 & 3593.533 & 0.888 & $1712,9.485 \mathrm{E}+05,163$ & 5119 \\
P-n50-k10 & 0.1618 & 4269.841 & 0.672 & $1837,9.980 \mathrm{E}+05,104$ & 1548 \\
\hline
\end{tabular}

TABLA NRO. 29. Instancias de baja complejidad matemática.

Elaboración propia.

Las siguientes instancias implementadas son definidas como de alta complejidad matemática, con mayor número de clientes que las instancias anteriores, alcanzando 200 clientes y 16 vehículos. Para estas instancias, se realizaron 5 ejecuciones de cada una con el fin de obtener respuestas en tiempos razonables. En la Tabla nro. 30 se presentan los resultados obtenidos.

\begin{tabular}{cccccc} 
Instancia & Hipervolumen & Spacing & Spread & Referencia (Nadir) & Tiempo (s) \\
\hline P-n55-k7 & 0.1803 & 8563.883 & 0.9175 & $1827,1.036 \mathrm{E}+06,105$ & 2616 \\
P-n55-k15 & 0.0956 & 3090.795 & 0.810 & $2082,1.106 \mathrm{E}+06,144$ & 1025 \\
P-n60-k15 & 0.1023 & 4042.229 & 0.912 & $2195,1.245 \mathrm{E}+06,135$ & 1655 \\
P-n65-k10 & 0.1435 & 3677.336 & 0.774 & $2145,1.250 \mathrm{E}+06,131$ & 2194 \\
P-n70-k10 & 0.1582 & 4711.068 & 0.815 & $2354,1.414 \mathrm{E}+06,135$ & 4828 \\
P-n76-k5 & 0.2196 & 11154.424 & 0.840 & $2280,1.548 \mathrm{E}+06,219$ & 1688 \\
P-n101-k4 & 0.1773 & 9263.594 & 0.934 & $3260,2.030 \mathrm{E}+06,413$ & 5485 \\
M-n151-k12 & 0.5006 & 20308.469 & 0.926 & $4335,3.225 \mathrm{E}+06,217$ & 1441 \\
M-n200-k16 & 0.1988 & 16883.001 & 0.948 & $6033,4.208 \mathrm{E}+06,304$ & 484416 \\
\hline
\end{tabular}

TABLA NRO. 30. Instancias de alta complejidad matemática. Elaboración propia.

Los valores de hipervolumen sirven de referencia para futuras investigaciones que utilicen otro tipo de metodologías de solución. La métrica de espaciamiento da una referencia de la dispersión entre las propias soluciones de un frente. Respecto a la métrica Spread, su valor indica que los frentes no son totalmente continuos con una aceptable distribución de las soluciones en el frente. Los valores se encuentran en un rango entre 0.67 y 1.17. Los frentes discontinuos y con soluciones aisladas tienden a tener un valor de esta métrica por encima de 1. Los tiempos obtenidos en las ejecuciones son considerados muy bajos en relación con el tipo de problema que resuelve con dos tipos de flota y tres objetivos.

De acuerdo a las soluciones obtenidas, la metodología utilizada muestra eficiencia para obtener soluciones al problema del GVRPPCWE ya que, en tiempos razonables, puede construir frentes de Pareto de aceptable propagación; y, con soluciones extremas para el objetivo del costo, cercanas al valor óptimo, verificado con modelos exactos en 
Toro-Ocampo et al. (2016). Los siguientes frentes de Pareto, desde la Figura nro. 39 hasta la 59, fueron presentados en las tablas de resultados nro. 29 y 30 .

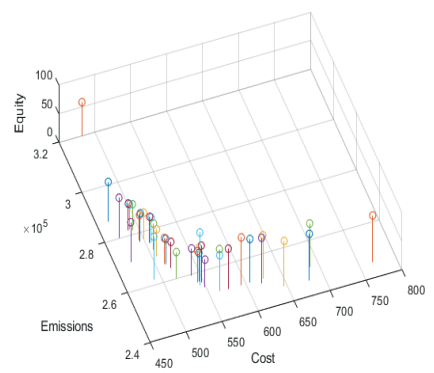

Figura NRO. 39. $P$-n16-k8.

Elaboración propia.

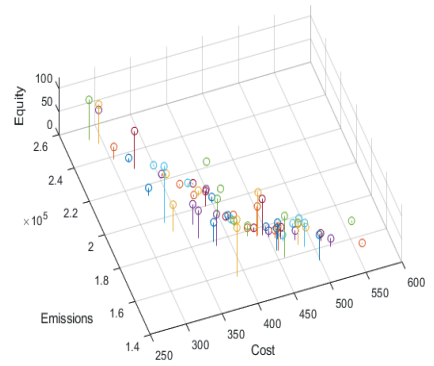

Figura NRO. 40. $P-n 19-k 2$.

Elaboración propia.

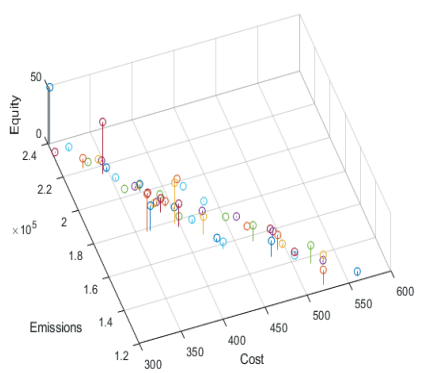

Figura NRO. 41. $P$-n20-k2.

Elaboración propia.

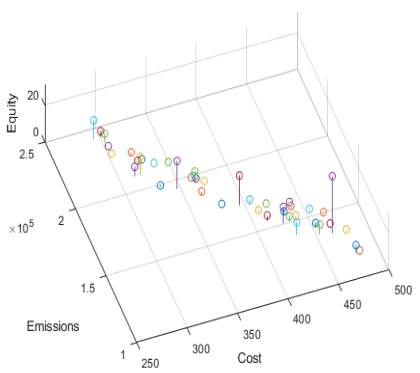

Figura NRo. 42. P-n21-k2.

Elaboración propia.

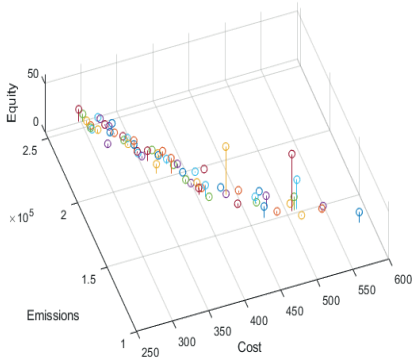

Figura NRO. 43. $P-n 22-k 2$.

Elaboración propia.

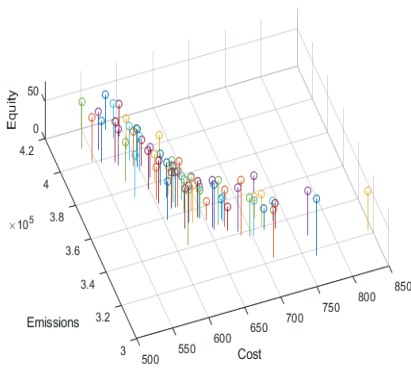

FIGURA NRO. 44. P-n23-k8.

Elaboración propia. 
Introducción al problema de enrutamiento de vehículos en la logística de distribución

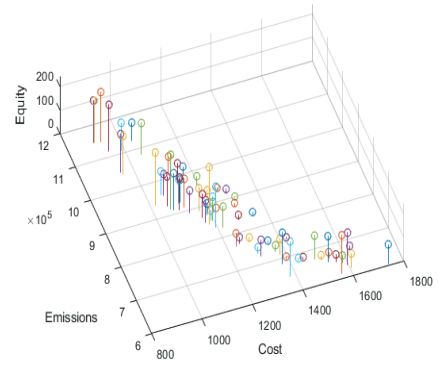

Figura NRO. 45. A-n32-k5.

Elaboración propia.

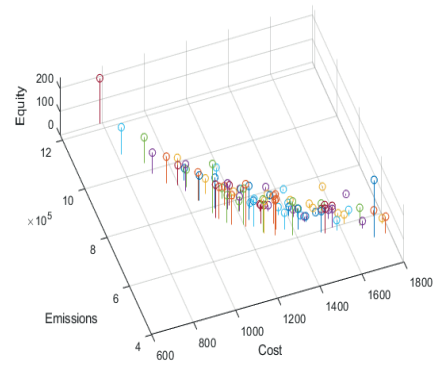

Figura NRO. 46. A-n33-k5. Elaboración propia.

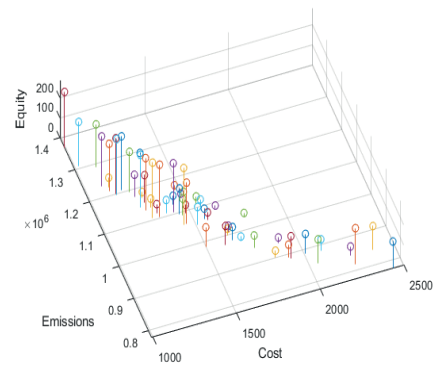

FIGURA NRO. 47. A-n39-k6. Elaboración propia.

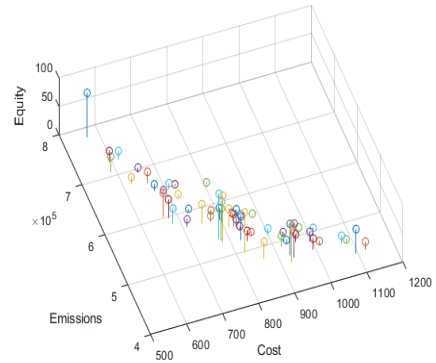

Figura NRO. 48. P-n40-k5.

Elaboración propia.

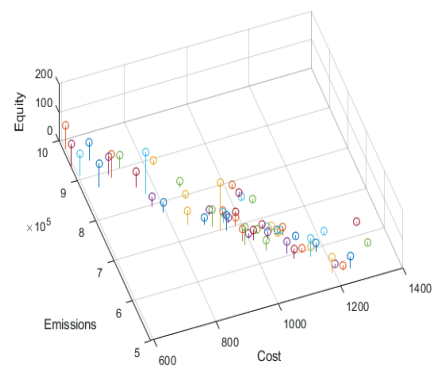

Figura NRO. 49. P-n45-k5.

Elaboración propia.

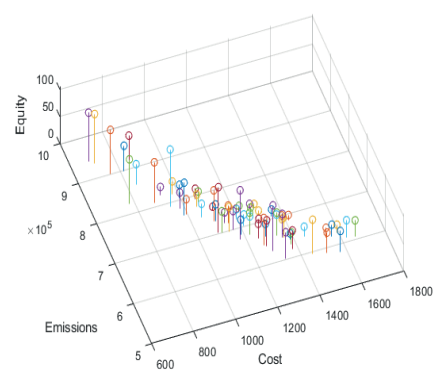

Figura NRo. 50. P-n50-k7.

Elaboración propia. 


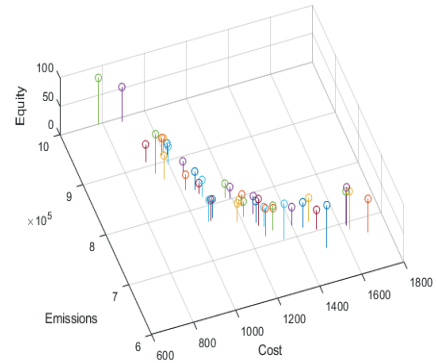

FIGURA NRO. 51. P-n50-k10.

Elaboración propia.

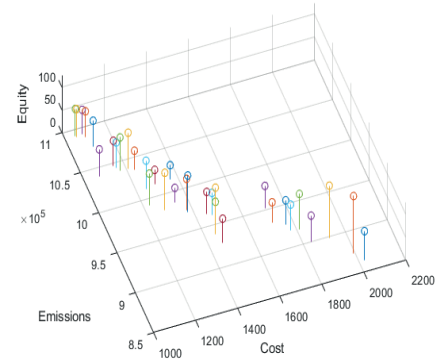

FigURA NRO. 52. $P-n 55-k 15$. Elaboración propia.

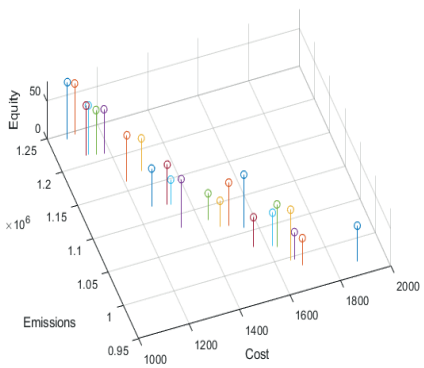

FIGURA NRO. 53. P-n60-k15. Elaboración propia.

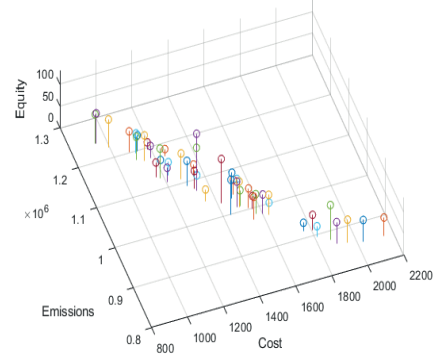

FIGURA NRO. 54. P-n65-k10.

Elaboración propia.

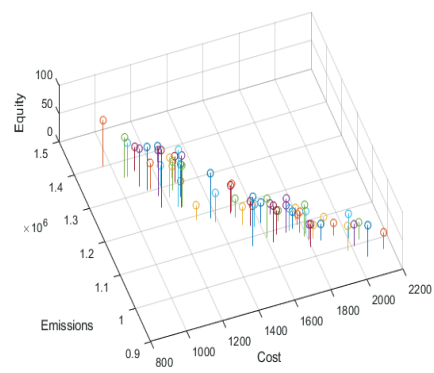

Figura NRO. 55. $P-n 70-k 10$. Elaboración propia.

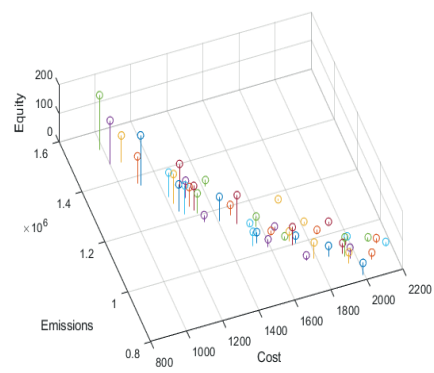

Figura NRO. 56. P-n76-k5. Elaboración propia. 
Introducción al problema de enrutamiento de vehículos en la logística de distribución

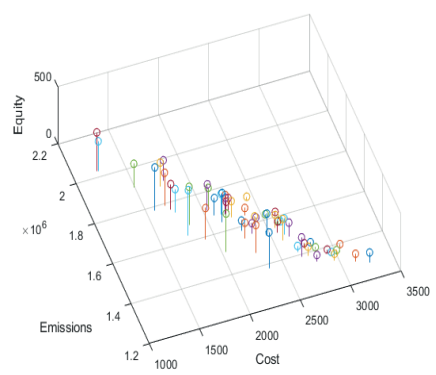

FIGURA NRO. 57. P-n101- $k 4$.

Elaboración propia.

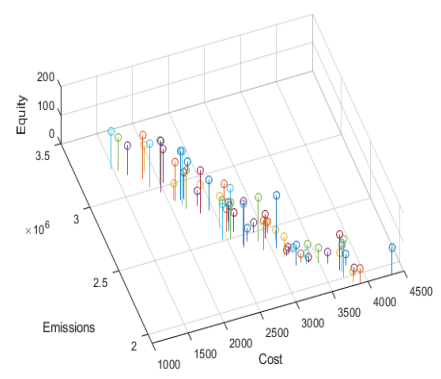

FIGURA NRO. 58. M-n151k12. Elaboración propia.

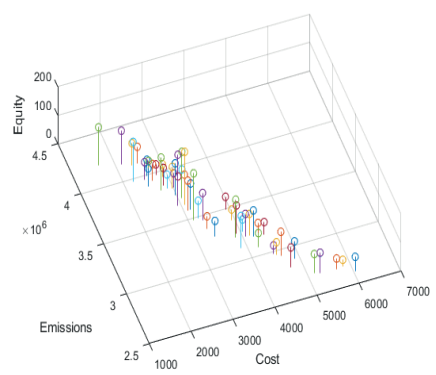

Figura NRO. 59. M-n200-k16.

Elaboración propia. 


\section{REFERENCIAS}

Aardal, K.; Pochet, Y. y Wolsey, L. A. (1995). Capacitated Facility Location: Valid Inequalities and Facets. Mathematics of Operations Research, 20(3), 562-582. https://doi.org/10.1287/moor.20.3.562.

Aardal, K.; Van Den Berg, P. L.; Gijswijt, D. y Li, S. (2015). Approximation Algorithms for Hard Capacitated Kfacility Location Problems. European fournal of Operational Research, 242(2), 358-368. https://doi.org/10.1016/j.ejor. 2014.10.011.

Ahn, J.; De Weck, O.; Geng, Y. y Klabjan, D. (2012). Column Generation Based Heuristics for a Generalized Location Routing Problem with Profits Arising in Space Exploration. European fournal of Operational Research, 223(1), 47-59. https://doi.org/10.1016/j.ejor.2012.06.018.

Ai, T. J. y Kachitvichyanukul, V. (2009). Particle Swarm Optimization and Two Solution Representations for Solving the Capacitated Vehicle Routing Problem. Computers \& Industrial Engineering, 56(1), 380-387. https://doi.org/10. 1016/j.cie.2008.06.012.

AKCA, Z.; Berger, R. y Ralphs, T. (2009). A Branch-And-Price Algorithm for Combined Location and Routing Problems Under Capacity Restrictions. En J. W. Chinneck; B. Kristjansson y M. J. Saltzman (eds). Operations Research and Cyber-Infrastructure. Operations Research (pp. 309-330). Springer.

Albareda-Sambola, M.; Fernández, E. y Laporte, G. (2007). Heuristic and Lower Bound for a Stochastic LocationRouting Problem. European Fournal of Operational Research, 179(3), 940-955. https://doi.org/10.1016/j.ejor.2005.04. 051 .

Alenezy, E. J. (2020). Solving Capacitated Facility Location Problem Using Lagrangian Decomposition and Volume Algorithm. Advances in Operations Research, 2020, 1-7. https://doi.org/10.1155/2020/5239176.

Alinaghian, M. y Shokouni, N. (2018). Multi-depot Multi-compartment Vehicle Routing Problem, Solved by a Hybrid Adaptive Large Neighborhood Search. Omega, 76, 85-99. https://doi.org/10.1016/j.omega.2017.05.002.

Alvim, A. C. y Taillard, E. D. (2013). Popmusic for the World Location-Routing Problem. EURO fournal on Transportation and Logistics, 2(3), 231-254. https://doi.org/10.1007/s13676-013-0024-2.

Ambrosino, D.; Sciomachen, A. y Scutellà, M. G. (2009). A Heuristic Based on Multi-exchange Techniques for a Regional Fleet Assignment Location-routing Problem. Computers \& Operations Research, 36(2), 442-460. https: //doi.org/10.1016/j.cor.2007.09.012.

Andre, M.; Hickman, A. J.; Hassel, D. y Joumard, R. (1995). Driving Cycles for Emission Measurements Under European Conditions. [Reporte técnico 950926]. SAE Technical Paper Series.

Androutsopoulos, K. N. y Zografos, K. G. (2012). A Bi-Objective Time-Dependent Vehicle Routing and Scheduling Problem for Hazardous Materials Distribution. EURO Journal on Transportation and Logistics, 1(1-2), 157-183. https: //doi.org/10.1007/s13676-012-0004-y.

Arague, J. R.; Hall, L. A. y Magnanti, T. L. (1990). Capacitated Trees Capacitated Routing and Associated Polyhedral. [Reporte técnico OR 232-90]. Massachusetts Institute of Technology, Operations Research Center. 
Archetti, C.; Desaulniers, G. y Speranza, M. G. (2017). Minimizing the Logistic Ratio in the Inventory Routing Problem. EURO fournal on Transportation and Logistics, 6(4), 289-306. https://doi.org/10.1007/s13676-016-0097-9.

Archetti, C.; Speranza, M. G. y Vigo, D. (2014). Chapter 10: Vehicle Routing Problems With Profits. En P. Toth y D. Vigo (eds). Vehicle Routing: Problems, Methods, and Applications (pp. 273-297). $2^{\text {da }}$ ed. SIAM.

Augerat, P.; Belenguer, J.-M.; Benavent, E.; Corbéran, A. y Naddef, D. (1998). Separating Capacity Constraints in the CVRP Using Tabu Search. European fournal of Operational Research, 106(2-3), 546-557. https://doi.org/10. 1016/S0377-2217(97)00290-7.

Azi, N.; Gendreau, M. y Potvin, J. Y. (2009). An Exact Algorithm for a Single-Vehicle Routing Problem With Time Windows and Multiple Routes. European fournal Operation Research, 178(3), 755-766. https://doi.org/10.1016/j.ejor. 2006.02.019.

Bader, J. y Zitzler, E. (2011). HypE: An Algorithm for Fast Hypervolume-Based Many-Objective Optimization. Evolutionary computation, 19(1), 45-76. https://doi.org/10.1016/j.apm.2016.01.059.

Bae, H. y Moon, I. (2016). Multi-Depot Vehicle Routing Problem with Time Windows Considering Delivery and Installation Vehicles. Applied Mathematical Modelling, 40(13-14), 6536-6549. https://doi.org/10.1016/j.apm.2016.01. 059.

Baker, B. y Ayechew, M. (2002). A Genetic Algorithm for the Vehicle Routing Problem. Computers and Operational Research, 30(5), 787-800. https://doi.org/10.1016/S0305-0548(02)00051-5.

Baldacci, R.; Bartolini, E.; Mingozzi, A. y Valletta, A. (2011a). An Exact Algorithm for the Period Routing Problem. Operations research, 59(1), 228-241. https://doi.org/10.1287/opre.1100.0875.

Baldacci, R.; Hadjiconstantinou, E. y Mingozzi, A. (2004). An Exact Algorithm for the Capacitated Vehicle Routing Problem Based on a Two-Commodity Network Flow Formulation. Operations research, 52(5), 723-738. https://doi.org/10.1287/opre.1040.0111.

Baldacci, R. y Mingozzi, A. (2009). A Unified Exact Method for Solving Different Classes of Vehicle Routing Problems. Mathematical Programming, 120(2), 347-380. https://doi.org/10.1007/s10107-008-0218-9.

Baldacci, R.; Mingozzi, A. y Roberti, R. (2011b). New Route Relaxation and Pricing Strategies for VRP. Operational Research, 59(5), 1269-1283. https://doi.org/10.1287/opre.1110.0975.

Ball, M. O.; Golden, B. L.; Assad, A. A. y Bodin, L. D. (1983). Planning for Truck Fleet Size in the Presence of a Common-Carrier Option. Decision Sciences, 14(1), 103-120. https://doi.org/10.1111/j.1540-5915.1983.tb00172.x.

Barreto, S.; Ferreira, C.; Paixao, J. y Santos, B. S. (2007). Using Clustering Analysis In A Capacitated LocationRouting Problem. European fournal of Operational Research, 179(3), 968-977. https://doi.org/10.1016/j.ejor.2005.06. 074.

Bashiri, M.; Rasoulinejad, Z. y Fallahzade, E. (2016). A New Approach on Auxiliary Vehicle Assignment in Capacitated Location Routing Problem. International Journal of Systems Science, 47(4), 886-902. https://doi.org/10. 1080/00207721.2014.906770.

Beasley, J. E. (1983). Route First-Cluster Second Methods for Vehicle Routing. Omega, 11(4), 403-408. https://doi.org/ 10.1016/0305-0483(83)90033-6.

Beirigo, B.; Schulte, F. y Negenborn, R. R. (2019). A Business Class for Autonomous Mobility-on-Demand: Modeling Service Quality Constraints in Ridesharing Systems. [En prensa]. https:/www.researchgate.net/publication/ 332241717_A_Business_Class_for_Autonomous_Mobility-on-Demand_Modeling_Service_Quality_Contracts_ in_Ridesharing_Systems.

Bektaş, T.; Ehmke, J. F.; Psaraftis, H. N. y Puchinger, J. (2019). The Role of Operational Research in Green Freight Transportation. European fournal of Operational Research, 274(3), 807-823. https://doi.org/10.1016/j.ejor.2018.06.001. 
Bektaş, T. y Laporte, G. (2011). The Pollution-routing Problem. Transportation Research Part B: Methodological, 45(8), 1232-1250. https://doi.org/10.1016/j.trb.2011.02.004.

Bektas, T.; Repoussis, P. y Tarantilis, C. (2014). Chapter 11: Dynamic Vehicle Routing Problems. En P. Toth y D. Vigo (eds). Vehicle Routing: Problems, Methods, and Applications (pp. 326-329). $2^{\text {da }}$ ed. SIAM.

Belenguer, J.-M.; Benavent, E.; Prins, C.; Prodhon, C. y Calvo, R. W. (2011). A Branch-and-Cut Method for the Capacitated Location-Routing Problem. Computers \& Operations Research, 38(6), 931-941. https://doi.org/10.1016/j. cor.2010.09.019.

Belgin, O.; Karaoglan, I. y Altiparmak, F. (2018). Two-echelon Vehicle Routing Problem with Simultaneous Pickup and Delivery: Mathematical Model and Heuristic Approach. Computers \& Industrial Engineering, 115, 1-16. https: //doi.org/10.1016/j.cie.2017.10.032

Bell, W. J.; Dalberto, L. M.; Fisher, M. L.; Greenfield, A. J.; Jaikumar, R.; Kedia, P.; Mack, R. G. y Prutzman, P. J. (1983). Improving the Distribution Of Industrial Gases With An On-Line Computerized Routing and Scheduling Optimizer. Interfaces, 13(6), 4-23. https://doi.org/10.1287/inte.13.6.4.

Berger, R. T.; Coullard, C. R. y Daskin, M. S. (2007). Location-Routing Problems with Distance Constraints. Transportation Science, 41(1), 29-43. https://doi.org/10.1287/trsc.1060.0156.

Bernal-Moyano, J. A.; Escobar, J. W.; Marín-Moreno, C.; Linfati, R. y Gatica, G. (2017). A Comparison of Trajectory Granular Based Algorithms for the Location-Routing Problem with Heterogeneous Fleet (LRPH). Dyna, 84(200), 193-201. https://doi.org/10.15446/dyna.v84n200.55533.

Bertsimas, D. J. (1992). A Vehicle Routing Problem with Stochastic Demand. Operations Research, 40(3), 574-585. https://doi.org/10.1287/opre.40.3.574.

Birge, J. R. y Louveaux, F. (2011). Introduction to Stochastic Programming. Springer Science \& Business Media.

Blakeley, F.; Argüello, B.; Cao, B.; Hall, W. y Knolmajer, J. (2003). Optimizing Periodic Maintenance Operations for Schindler Elevator Corporation. Interfaces, 33(1), 67-79. https://doi.org/10.1287/inte.33.1.67.12722.

BLocho, M. (2020). Heuristics, Metaheuristics, and Hyperheuristics for Rich Vehicle Routing Problems. En J. NALEPA (ed). Smart Delivery Systems (pp. 101-156). Elsevier.

Bodin, L. (1975). A Taxonomic Structure for Vehicle Routing and Scheduling Problem. Computers and Urban Society, 1(1), 11-29. https://doi.org/10.1016/0305-7097(75)90003-4.

Bolanos, R.; Toro-Ocampo, E. y Granada, M. (2016). A Population-based Algorithm for the Multi Travelling Salesman Problem. International fournal of Industrial Engineering Computations, 7(2), 245-256. https://doi.org/10. 5267/j.jijiec.2015.10.005.

Bolduc, M.-C.; Renaud, J. y Boctor, F. (2007). A Heuristic for the Routing and Carrier Selection Problem. European fournal of Operational Research, 183(2), 926-932. https://doi.org/10.1016/j.ejor.2006.10.013.

Bolduc, M.-C.; Renaud, J.; Boctor, F. y Laporte, G. (2008). A Perturbation Metaheuristic for the Vehicle Routing Problem with Private Fleet and Common Carriers. Fournal of the Operational Research Society, 59(6), 776-787. https: //doi.org/10.1057/palgrave.jors.2602390.

Bornstein, C. T. y Azlan, H. B. (1998). The Use of Reduction Tests and Simulated Annealing for the Capacitated Location Problem. Location Science, 6(1-4), 67-81. https://doi.org/10.1016/S0966-8349(98)00062-X.

Bosman, P. y Thierens, D. (2002). Multi-objective Optimization with Diversity Preserving Mixture-based Iterated Density Estimation Evolutionary Algorithms. International fournal of Approximate Reasoning, 31(3), 259-289. https: //doi.org/10.1016/S0888-613X(02)00090-7. 
Bouhafs, L.; HajJam, A. y Koukam, A. (2006). A Combination of Simulated Annealing and Ant Colony System for the Capacitated Location-routing Problem. En B. GABRys; R. HowletT y L. JaIN (eds). International Conference on Knowledge-Based and Intelligent Information and Engineering Systems (pp. 409-416). Springer.

Boyer, K. K.; Prud'homme, A. M. y Chung, W. (2009). The Last Mile Challenge: Evaluating the Effects of Customer Density and Delivery Window Patterns. Journal of business logistics, 30(1), 185-201. https://doi.org/10.1002/j. 2158-1592.2009.tb00104.x.

Bradstreet, L. (2011). The Hypervolume Indicator for Multi-objective Optimisation: Calculation and Use. [Tesis Doctoral, University of Western Australia Perth]. Repositorio UWA. https://research-repository.uwa.edu.au/files/ 3236619/Bradstreet_Lucas_2011.pdf.

Braekers, K.; Ramaekers, K. y Van Nieuwenhuyse, I. (2016). The Vehicle Routing Problem: State of the Art Classification and Review. Computers \& Industrial Engineering, 99, 300-313. https://doi.org/10.1016/j.cie.2015.12.007.

Breedam, A. (1995). Improvement Heuristics for the Vehicle Routing Problem Based on Simulated Annealing. European Fournal of Operational Research, 86, 480-490. https://doi.org/10.1016/0377-2217(94)00064-J.

Breunig, U.; Baldacci, R.; Hartl, R. F. y Vidal, T. (2019). The Electric Two-echelon Vehicle Routing Problem. Computers \& Operations Research, 103, 198-210. https://doi.org/10.1016/j.cor.2018.11.005.

Breunig, U.; Schmid, V.; HarTl, R. F. y Vidal, T. (2016). A Large Neighbourhood Based Heuristic for Two-echelon Routing Problems. Computers \& Operations Research, 76, 208-225. https://doi.org/10.1016/j.cor.2016.06.014.

Brown, G. G.; Ellis, C. J.; Graves, G. W. y Ronen, D. (1987). Real-time, Wide Area Dispatch of Mobil Tank Trucks. Interfaces, 17(1), 107-120. https://doi.org/10.1287/inte.17.1.107.

Bruno, G.; Diglio, A.; Melisi, A. y Piccolo, C. (2017). A Districting Model to Support the Redesign Process of Italian Provinces. En A. SForza y C. STERLE (eds). International conference on optimization and decision science (pp. 245-256). Springer.

Burks, R. (2006). An Adaptive Tabu Search Heuristic For The Location Routing Pickup And Delivery Problem With Timewindows With A Theater Distribution Application. [Tesis Doctoral, Graduate School Of Engineering And Management, Air Force Institute Of Technology, Ohio]. Repositorio AFIT Scholar. https://scholar.afit.edu/etd/ $3302 /$.

Caballero, R.; González, M.; Guerrero, F. M.; Molina, J. y Paralera, C. (2007). Solving a Multiobjective Location Routing Problem with a Metaheuristic Based on Tabu Search. Application to a Real Case in Andalusia. European fournal of Operational Research, 177(3), 1751-1763. https://doi.org/10.1016/j.ejor.2005.10.017.

CAI, Z.; Zhang, Z. y He, H. (2018). Solving the Last Mile Delivery Problem Using Iterated Local Search Approach. En: 2018 IEEE 15th International Conference on Networking, Sensing and Control (ICNSC) (pp. 1-6). IEEE. https://doi. org/10.1109/ICNSC.2018.8361271.

Campbell, A. M.; Vandenbussche, D. y Hermann, W. (2008). Routing for Relief Efforts. Transportation Science, 42(2), 127-145. https://doi.org/10.1287/trsc.1070.0209.

Carranza-Huartos, E. (2019). Logística 4.0: Importancia En el Proceso Logístico de Distribución de Última Milla. [Proyecto final especialización, Universidad Militar Nueva Granada]. Repositorio Universidad Militar Nueva Granada. https://repository.unimilitar.edu.co/handle/10654/31727.

Cassidy, P. y Bennetr, H. (1972). TRAMP-a Multi-depot Vehicle Scheduling System. Fournal of the Operational Research Society, 23(2), 151-163. https://doi.org/10.1057/jors.1972.26.

CAstaneda, J. F.; Toro, E. M. y Gallego, R. A. (2020). Iterated Local Search for the Vehicle Routing Problem with a Private Fleet and a Common Carrier. Engineering Optimization, 52(10), 1796-1813. https://doi.org/10.1080/0305215X. 2019.1681985. 
Castaño, A. O.; Toro-Ocampo, E. M. y Gallego-Rendón, R. A. (2020). Análisis de Sensibilidad para el MDVRPPC Multi-Objetivo que Considera Costo e Impacto Ambiental. Revista colombiana de tecnologías de avanzada (RCTA), 2(34), 1-8. https://doi.org/10.24054/16927257.v34.n34.2019.3858.

ÇEtiner, S.; Sepil, C. y Süral, H. (2010). Hubbing and Routing in Postal Delivery Systems. Annals of Operations research, 181(1), 109-124. https://doi.org/10.1007/s10479-010-0705-2.

Chan, Y. y Baker, S. F. (2005). The Multiple Depot, Multiple Traveling Salesmen Facility-location Problem: Vehicle Range, Service Frequency, and Heuristic Implementations. Mathematical and Computer Modelling, 41(8-9), 10351053. https://doi.org/10.1016/j.mcm.2003.08.011.

Chen, C.; QIu, R. y Hu, X. (2018). The Location-routing Problem with Full Truckloads in Low-carbon Supply Chain Network Designing. Mathematical Problems in Engineering, 2018. Artículo 6315631. https://doi.org/10.1155/2018/ 6315631.

Chen, C.-H. y Ting, C.-J. (2007). A Hybrid Lagrangian Heuristic/Simulated Annealing Algoritm For The Multi-Depot Location Routing Problem. En: Proceedings of the Eastern Asia Society for Transportation Studies Vol. 6 (The 7th International Conference of Eastern Asia Society for Transportation Studies, 2007) (pp. 137-151). Eastern Asia Society for Transportation Studies.

Chowdhury, P. K. R.; Weaver, J. E.; Weber, E. M.; Lunga, D.; St Thomas, M. L.; Rose, A. N. y Bhaduri, B. L. (2019). Electricity Consumption Patterns Within Cities: Application of a Data-driven Settlement Characterization Method. International fournal of Digital Earth, 13(1), 119-135. https://doi.org/10.1080/17538947.2018.1556355.

Christofides, N. y Beasley, J. E. (1983). Extensions to a Lagrangean Relaxation Approach for the Capacitated Warehouse Location Problem. European fournal of Operational Research, 12(1), 19-28. https://doi.org/10.1016/ 0377-2217(83)90179-0.

Christofides, N.; Mingozzi, A. y Toтн, P. (1981). Exact Algorithms for the Vehicle Routing Problem, Based on Spanning Tree and Shortest Path Relaxations. Mathematical programming, 20(1), 255-282. https://doi.org/10.1007/ BF01589353.

CHu, C.-W. (2005). A Heuristic Algorithm for the Truckload and Less-than-truckload Problem. European fournal of Operational Research, 165(3), 657-667. https://doi.org/10.1016/j.ejor.2003.08.067.

Chu, C.-W.; Chang, J.-R. y Chang, K.-H. (2019). A Heuristic Algorithm for the Multi-depot Vehicle Routing Problem with Outsider Carrier Selection. Fournal of Marine Science and Technology, 27(2), 170-183. https://doi.org/10.6119/ JMST.201904_27(2).0010.

Clarke, G. y Wright, J. W. (1964). Scheduling of Vehicles From a Central Depot to a Number of Delivery Points. Operations research, 12(4), 568-581. https://www.jstor.org/stable/167703.

Coelho, L. C.; Cordeau, J.-F. y Laporte, G. (2012). Consistency in Multi-vehicle Inventory-routing. Transportation Research Part C: Emerging Technologies, 24, 270-287. https://doi.org/10.1016/j.trc.2012.03.007.

Cohon, J. L. y Marks, D. H. (1975). A Review and Evaluation of Multiobjective Programing Techniques. Water resources research, 11(2), 208-220. https://doi.org/10.1029/WR011i002p00208.

Contardo, C.; Cordeau, J.-F. y Gendron, B. (2013). A Computational Comparison of Flow Formulations for the Capacitated Location-Routing Problem. Discrete Optimization, 10(4), 263-295. https://doi.org/10.1016/j.disopt.2013. 07.005 .

Contardo, C.; Cordeau, J.-F. y Gendron, B. (2014a). An Exact Algorithm Based on Cut-and-Column Generation for the Capacitated Location-Routing Problem. INFORMS Journal on Computing, 26(1), 88-102. https://doi.org/10.1287/ ijoc.2013.0549.

Contardo, C.; Cordeau, J.-F. y Gendron, B. (2014b). A GRASP + ILP-based Metaheuristic for the Capacitated Location-routing Problem. fournal of Heuristics, 20(1), 1-38. https://doi.org/10.1007/s10732-013-9230-1. 
Contardo, C. y Martinelli, R. (2014). A New Exact Algorithm for the Multi-depot Vehicle Routing Problem Under Capacity and Route Length Constraints. Discrete Optimization, 12, 129-146. https://doi.org/10.1016/j.disopt.2014.03. 001.

Cordeau, J.-F.; Gendreau, M. y Laporte, G. (1997). A Tabu Search Heuristic for Periodic and Multi-depot Vehicle Routing Problems. Networks: An International fournal, 30(2), 105-119. https://doi.org/10.1002/(SICI) 1097-0037(199709)30:2<105::AID-NET5>3.0.CO;2-G.

Cordeau, J.-F. y Maischberger, M. (2012). A Parallel Iterated Tabu Search Heuristic for Vehicle Routing Problems. Computers \& Operations Research, 39(9), 2033-2050. https://doi.org/10.1016/j.cor.2011.09.021.

CôtÉ, J.-F. y Potvin, J.-Y. (2009). A Tabu Search Heuristic for the Vehicle Routing Problem with Private Fleet and Common Carrier. European fournal of Operational Research, 198(2), 464-469. https://doi.org/10.1016/j.ejor.2008.09. 009.

Crainic, T. G.; Mancini, S.; Perboli, G. y Tadei, R. (2011). Multi-start Heuristics for the Two-echelon Vehicle Routing Problem. En P. Merz y J.-K. Hao (eds). European Conference on Evolutionary Computation in Combinatorial Optimization (pp. 179-190). Springer.

Crainic, T. G.; Mancini, S.; Perboli, G. y Tadei, R. (2013). GRASP with Path Relinking for the Two-echelon Vehicle Routing Problem. En L. Di Gaspero; A. Schaerf y T. StÜtzle (eds). Advances in Metaheuristics (pp. 113-125). Springer.

Crainic, T. G.; Perboli, G.; Mancini, S. y Tadei, R. (2010). Two-echelon Vehicle Routing Problem: a Satellite Location Analysis. Procedia-Social and Behavioral Sciences, 2(3), 5944-5955. https://doi.org/10.1016/j.ejor.2008.09.009.

Crainic, T. G.; Ricciardi, N. y Storchi, G. (2004). Advanced Freight Transportation Systems for Congested Urban Areas. Transportation Research Part C: Emerging Technologies, 12(2), 119-137. https://doi.org/10.1016/j.trc.2004.07. 002.

Crevier, B.; Cordeau, J.-F. y Laporte, G. (2007). The Multi-depot Vehicle Routing Problem with Inter-depot Routes. European journal of operational research, 176(2), 756-773. https://doi.org/10.1016/j.ejor.2005.08.015.

Cuda, R.; Guastaroba, G. y Speranza, M. G. (2015). A Survey on Two-echelon Routing Problems. Computers \& Operations Research, 55, 185-199. https://doi.org/10.1016/j.cor.2014.06.008.

Dabia, S.; LAI, D. y Vigo, D. (2019). An Exact Algorithm for a Rich Vehicle Routing Problem with Private Fleet and Common Carrier. Transportation Science, 53(4), 986-1000. https://doi.org/10.1287/trsc.2018.0852.

Deb, K. (2014). Multi-objective Optimization. En E. Burke y G. Kendall (eds). Search Methodologies (pp. 403-449). Springer.

Deb, K.; Pratap, A.; Agarwal, S. y Meyarivan, T. (2002). A Fast and Elitist Multiobjective Genetic Algorithm: NSGAII. IEEE transactions on evolutionary computation, 6(2), 182-197. https://doi.org/10.1109/4235.996017.

Dellaert, N.; Dashty Saridaro, F.; Van Woensel, T. y Crainic, T. G. (2019). Branch-and-price-based Algorithms for the Two-echelon Vehicle Routing Problem with Time Windows. Transportation Science, 53(2), 463-479. https: //doi.org/10.1287/trsc.2018.0844.

Dell'Amico, M. y Hadjidimitriou, S. (2012). Innovative Logistics Model and Containers Solution for Efficient Last Mile Delivery. Procedia-Social and Behavioral Sciences, 48, 1505-1514. https://doi.org/10.1016/j.sbspro.2012.06.1126.

Demir, E.; Bektaş, T. y Laporte, G. (2012). An Adaptive Large Neighborhood Search Heuristic for the Pollutionrouting Problem. European Fournal of Operational Research, 223(2), 346-359. https://doi.org/10.1016/j.ejor.2012.06. 044.

Demir, E.; Bektaş, T. y Laporte, G. (2014). A Review of Recent Research on Green Road Freight Transportation. European fournal of Operational Research, 237(3), 775-793. https://doi.org/10.1016/j.ejor.2013.12.033. 
Derbel, H.; Jarboui, B.; Chabchoub, H.; Hanafi, S. y Mladenović, N. (2011). A Variable Neighborhood Search for the Capacitated Location-routing Problem. En: 2011 4th International Conference on Logistics (pp. 514-519). IEEE. https://doi.org/10.1109/LOGISTIQUA.2011.5939452.

Domschke, W. y Drexl, A. (1985). Add-heuristics Starting Procedures for Capacitated Plant Location Models. European fournal of Operational Research, 21, 47-53. https://doi.org/10.1016/0377-2217(85)90086-4.

Doong, S.; LaI, C. y Wu, C. (2007). Genetic Subgradient Method for Solving Location-allocation Problems. Applied Soft Computing, 7(1), 373-386. https://doi.org/10.1016/j.asoc.2005.06.008.

Drexl, M. y Schneider, M. (2015). A Survey of Variants and Extensions of the Location-routing Problem. European Journal of Operational Research, 241(2), 283-308. https://doi.org/10.1016/j.ejor.2014.08.030.

Du, J.; LI, X.; Yu, L.; Ralescu, D. y Zhou, J. (2017). Multi-depot Vehicle Routing Problem for Hazardous Materials Transportation: A Fuzzy Bilevel Programming. Information Sciences, 399, 201-218. https://doi.org/10.1016/j.ins. 2017.02.011.

Dueck, G. y Scheuer, T. (1990). Threshold Accepting: a General Purpose Optimization Algorithm Appearing Superior to Simulated Annealing. Journal of computational physics, 90(1), 161-175. https://doi.org/10.1016/0021-9991(90) 90201-B.

Duhamel, C.; Lacomme, P.; Prins, C. y Prodhon, C. (2008). A Memetic Approach for the Capacitated Location Routing Problem. En: Proceedings of the 9th EU/Meeting on Metaheuristics for Logistics and Vehicle Routing, 38 (pp. 39-48). https://hal.archives-ouvertes.fr/hal-02081981.

Duhamel, C.; Lacomme, P.; Prins, C. y Prodhon, C. (2010). A GRASP $\times$ ELS Approach for the Capacitated Locationrouting Problem. Computers \& Operations Research, 37(11), 1912-1923. https://doi.org/10.1016/j.cor.2009.07.004.

Ehrgott, M. y Gandibleux, X. (2003). Multiobjective Combinatorial Optimization-theory, Methodology, and Applications. En M. EhrgotT y X. GAndibleux (eds). Multiple criteria optimization: State of the art annotated bibliographic surveys (pp. 369-444). Springer.

Eitzen, H.; Lopez-Pires, F.; Baran, B.; Sandoya, F. y Chicaiza, J. L. (2017). A Multi-objective Two-echelon Vehicle Routing Problem. An Urban Goods Movement Approach for Smart City Logistics. En: 2017 XLIII Latin American Computer Conference (CLEI) (pp. 1-10). IEEE. https://doi.org/10.1109/CLEI.2017.8226454.

Eksioglu, B.; Vural, A. V. y Reisman, A. (2009). The Vehicle Routing Problem: A Taxonomic Review. Computers \& Industrial Engineering, 57(4), 1472-1483. https://doi.org/10.1016/j.cie.2009.05.009.

Ellegood, W. A.; Solomon, S.; North, J. y Campbell, J. F. (2020). School Bus Routing Problem: Contemporary Trends and Research Directions. Omega, 95. Artículo nro. 102056. https://www.sciencedirect.com/science/article/ pii/S0305048318305127.

ElshaER, R. y Awad, H. (2020). A Taxonomic Review of Metaheuristic Algorithms for Solving the Vehicle Routing Problem and Its Variants. Computers \& Industrial Engineering, 140. Artículo nro. 106242. https://doi.org/10.1016/j. cie.2019.106242.

Escobar, J. W.; Linfati, R. y Jaimes, W. A. (2015). Problema de localización y ruteo con restricciones de capacidad: Revisión de la literatura. Revista Facultad de Ingeniería, 24(39), 85-98. https://www.redalyc.org/articulo.oa?id= 413940776008

Escobar, J. W.; Linfati, R. y Tотн, P. (2013). A Two-phase Hybrid Heuristic Algorithm for the Capacitated Locationrouting Problem. Computers \& Operations Research, 40(1), 70-79.

Escobar, J. W.; Linfati, R.; Toth, P. y Baldoduin, M. G. (2014). A Hybrid Granular Tabu Search Algorithm for the Multi-depot Vehicle Routing Problem. Fournal of heuristics, 20(5), 483-509. https://doi.org/10.1007/ s10732-014-9247-0. 
Euchi, J. (2017). The Vehicle Routing Problem with Private Fleet and Multiple Common Carriers: Solution with Hybrid Metaheuristic Algorithm. Vehicular Communications, 9, 97-108. https://doi.org/10.1016/j.vehcom.2017.04.005.

Euchi, J.; Chabchoub, H. y Yassine, A. (2013). New Evolutionary Algorithm Based on 2-opt Local Search to Solve the Vehicle Routing Problem with Private Fleet and Common Carrier. En P.-Y. YIN (ed). Trends in Developing Metaheuristics, Algorithms, and Optimization Approaches (pp. 125-148). IGI Global.

Eveborn, P.; Flisberg, P. y Rönnqvist, M. (2006). Laps Care-an Operational System for Staff Planning of Home Care. European journal of operational research, 171(3), 962-976. https://doi.org/10.1016/j.ejor.2005.01.011.

Fathollahi-Fard, A. M.; Ahmadi, A.; Goodarzian, F. y Cheikhrouhou, N. (2020). A Bi-objective Home Healthcare Routing and Scheduling Problem Considering Patients' Satisfaction in a Fuzzy Environment. Applied soft computing, 93, 106385. https://doi.org/10.1016/j.asoc.2020.106385.

Feillet, D.; Dejax, P.; Gendreau, M. y Gueguen, C. (2004). An Exact Algorithm for the Elementary Shortest Path Problem with Resource Constraints: Application to Some Vehicle Routing Problems. Networks: An International Journal, 44(3), 216-229.

Figliozzi, M. (2010). Vehicle Routing Problem for Emissions Minimization. fournal of Transportation Research Board, 2197(1), 1-7. https://doi.org/10.3141/2197-01.

Fischetti, M.; Toth, P. y Vigo, D. (1994). A Branch-and-bound Algorithm for the Capacitated Vehicle Routing Problem on Directed Graphs. Operations Research, 42(5), 846-859. https://doi.org/10.1287/opre.42.5.846.

Fourer, R.; Gay, D. M. y Kernighan, B. W. (1990). A Modeling Language for Mathematical Programming. Management Science, 36(5), 519-554. https://doi.org/10.1287/mnsc.36.5.519.

Galindres-Guancha, L.; Toro-Ocampo, E. y Rendón, R. (2018). Multi-objective MDVRP Solution Considering Route Balance and Cost Using the ILS Metaheuristic. International fournal of Industrial Engineering Computations, 9(1), 33-46. https://doi.org/10.5267/j.ijiec.2017.5.002.

Gallego-Rendón, R. A.; Ocampo-Toro, E. M. y Escobar-Zuluaga, A. H. (2015). Técnicas Heurísticas y Metaheurísticas. Editorial Universidad Tecnológica de Pereira.

Gansterer, M. y Hartl, R. F. (2018). Collaborative Vehicle Routing: a Survey. European fournal of Operational Research, 268(1), 1-12. https://doi.org/10.1016/j.ejor.2017.10.023.

Gendreau, M.; Hertz, A. y Laporte, G. (1992). New Insertion and Postoptimization Procedures for the Traveling Salesman Problem. Operations Research, 40(6), 1086-1094. https://doi.org/10.1287/opre.40.6.1086.

Geoffrion, A. y McBride, R. (1978). Lagrangean Relaxation to Capacitated Facility Location Problems. AIIE, 10, 40-47. https://doi.org/10.1080/05695557808975181.

Ghiani, G.; Guerriero, F. y Musmanno, R. (1999). The Capacitated Plant Location Problem with Multiple Facilities in the Same Time. Computers and Operations Research, 29(13), 1093-1912. https://doi.org/10.1016/S0305-0548(01) 00065-X.

Gillett, B. y Miller, L. (1974). A Heuristic Algorithm for the Vehicle-dispatch Problem. Operational Research, 22(2), 340-349. https://doi.org/10.1287/opre.22.2.340.

Gimadi, E.; ShtepA, A. y Tsidulko, O. (2019). Improved Exact Algorithm for the Capacitated Facility Location Problem on a Line Graph. En: 2019 15th International Asian School-Seminar Optimization Problems of Complex Systems (OPCS) (pp. 53-57). IEEE. https://doi.org/10.1109/OPCS.2019.8880248.

Giosa, I.; Tansini, I. y Viera, I. (2002). New Assignment Algorithms for the Multi-depot Vehicle Routing Problem. Journal of the operational research society, 53(9), 977-984. https://doi.org/10.1057/palgrave.jors.2601426. 
Goeke, D.; Gschwind, T. y Schneider, M. (2019). Upper and Lower Bounds for the Vehicle-routing Problem with Private Fleet and Common Carrier. Discrete Applied Mathematics, 264, 43-61. https://doi.org/10.1016/j.dam.2018.10. 015 .

Golden, B.; Bodin, L.; Doyle, T. y Stewart, W. (1980). Approximate Traveling Salesman Algorithms. Operation Research, 28, 694-711. https://doi.org/10.1287/opre.28.3.694.

Golden, B. L.; Raghavan, S. y Wasil, E. A. (2008). The Vehicle Routing Problem: Latest Advances and New Challenges, Vol. 43. Springer Science \& Business Media.

Golden, B. L. y Wasil, E. A. (1987). OR Practice-Computerized Vehicle Routing in the Soft Drink Industry. Operations research, 35(1), 6-17.

Golden, B. L. y Wong, R. T. (1981). Capacitated Arc Routing Problems. Networks, 11(3), 305-315. https://doi.org/10. 1002/net.3230110308.

Grabenschweiger, J.; Tricoire, F. y Doerner, K. F. (2018). Finding the Trade-off Between Emissions and Disturbance in An Urban Context. Flexible Services and Manufacturing fournal, 30(3), 554-591. https://doi.org/10. 1007/s10696-017-9297-3.

Granada, M.; Toro, E. M. y Gallego, R. (2019). An MiP Formulation for the Open Location-routing Problem Considering the Topological Characteristic of the Solution-paths. Networks, 74(4), 374-388. https://doi.org/10.1002/ net.21912.

Grangier, P.; Gendreau, M.; Lehuédé, F. y Rousseau, L.-M. (2016). An Adaptive Large Neighborhood Search for the Two-echelon Multiple-trip Vehicle Routing Problem with Satellite Synchronization. European fournal of Operational Research, 254(1), 80-91. https://doi.org/10.1016/j.ejor.2016.03.040.

Groër, C.; Golden, B. y Wasil, E. (2010). A Library of Local Search Heuristics for the Vehicle Routing Problem. Mathematical Programming Computation, 2(2), 79-101. https://doi.org/10.1007/s12532-010-0013-5.

Guajardo, M. y RönnQvist, M. (2016). A Review on Cost Allocation Methods in Collaborative Transportation. International transactions in operational research, 23(3), 371-392. https://doi.org/10.1111/itor.12205.

Han, Y.-Q.; LI, J.-Q.; LiU, Z.; Liv, C. y Tian, J. (2020). Metaheuristic Algorithm for Solving the Multi-objective Vehicle Routing Problem with Time Window and Drones. International fournal of Advanced Robotic Systems, 17(2), 1-14. https://doi.org/10.1177/1729881420920031.

Hansen, P. H.; Hegedahl, B.; Hjortkjaer, S. y Obel, B. (1994). A Heuristic Solution to the Warehouse Locationrouting Problem. European fournal of Operational Research, 76(1), 111-127. https://doi.org/10.1016/0377-2217(94) 90010-8.

HARris, I.; Mumford, C. y Naim, M. (2014). A Hybrid Multi-objective Approach to Capacitated Facility Location with Wexible Store Allocation for Green Logistics Modeling. Transportation Research, 66, 1-22. 10.1016/j.tre.2014.01.010.

Hassan-Pour, H.; Mosadegh-Khah, M. y Tavaknoli-Moghaddam, R. (2009). Solving a Multi-objective Multi-depot Stochastic Location-routing Problem by a Hybrid Simulated Annealing Algorithm. Proceedings of the Institution of Mechanical Engineers, Part B: Journal of Engineering Manufacture, 223(8), 1045-1054. https://doi.org/10.1243/ $09544054 J \mathrm{JEM} 1349$.

Helsgaun, K. (2000). An Effective Implementation of the Lin-Kernighan Traveling Salesman Heuristic. European Journal of Operational Research, 126(1), 106-130. https://doi.org/10.1016/S0377-2217(99)00284-2.

Helsgaun, K. (2009). General K-opt Submoves for the Lin-Kernighan TSP Heuristic. Mathematical Programming Computation, 1(2-3), 119-163. https://doi.org/10.1007/s12532-009-0004-6. 
Helsgaun, K. (2015). Solving the Equality Generalized Traveling Salesman Problem Using the Lin-kernighanhelsgaun Algorithm. Mathematical Programming Computation, 7(3), 269-287. https://doi.org/10.1007/ s12532-015-0080-8.

Hinson, J. y Mulherkar, S. (1975). Improvements to the Clarke and Wright Algorithm as Applied to An Airline Scheduling Problem. [Reporte técnico]. Federal Express Corp.

Ho, W.; Ho, G. T. S.; Ji, P. y LaU, H. C. W. (2008). A Hybrid Genetic Algorithm for the Multi-depot Vehicle Routing Problem. Engineering Applications of Artificial Intelligence, 21(4), 548-557. 10.1016/j.engappai.2007.06.001.

Holland, C.; Levis, J.; Nuggehalli, R.; Santilli, B. y Winters, J. (2017). UPS Optimizes Delivery Routes. INFORMS fournal on Applied Analytic, 47(1), 8-23. https://doi.org/10.1287/inte.2016.0875.

Huang, M.; Smilowitz, K. y Balcik, B. (2012). Models for Relief Routing: Equity, Efficiency and Efficacy. Transportation research part E: logistics and transportation review, 48(1), 2-18. https://doi.org/10.1016/j.tre.2011.05. 004.

Huang, S.-H. (2015). Solving the Multi-compartment Capacitated Location Routing Problem with Pickup-delivery Routes and Stochastic Demands. Computers \& Industrial Engineering, 87, 104-113. https://doi.org/10.1016/j.cie.2015. 05.008 .

IBM-ILOG (2009). CPLEX Optimization Subroutine Library Guide and Reference. (version 12.1).

Jabali, O.; Leus, R.; Van Woensel, T. y De KoK, T. (2015). Self-imposed Time Windows in Vehicle Routing Problems. OR Spectrum, 37(2), 331-352. https://doi.org/10.1007/s00291-013-0348-1.

Jabali, O.; Van Woensel, T. y De KoK, A. (2012). Analysis of Travel Times and $\mathrm{CO}_{2}$ Emissions in Time-dependent Vehicle Routing. Production and Operations Management, 21(6), 1060-1074. https://doi.org/10.1111/j.1937-5956.2012. 01338.x.

Jacobsen, S. (1983). Heuristics for the Capacitated Plant Location Model. European fournal of Operational Research, 12, 253-261. https://doi.org/10.1016/0377-2217(83)90195-9.

Jacobsen, S. K. y Madsen, O. B. (1980). A Comparative Study of Heuristics for a Two-level Routing-location Problem. European Fournal of Operational Research, 5(6), 378-387. https://doi.org/10.1016/0377-2217(80)90124-1.

Jaimes, W. A.; Serna, M. D. A. y CÁrdenas, I. D. (2014). Comportamientos logísticos en la distribución de última milla de productos alimenticios en villavicencio, colombia. Revista EIA, 11(21), 145-166.

Janssens, J;; Van den Bergh, J.; Sörensen, K. y Cattrysse, D. (2015). Multi-objective Microzone-based Vehicle Routing for Courier Companies: From Tactical to Operational Planning. European fournal of Operational Research, 242(1), 222-231. https://doi.org/10.1016/j.ejor.2014.09.026.

Jaramillo, J. (2011, octubre 5-7). The Green Vehicle Routing Procedure. En: Proceedings, informs annual meeting, Myrtle Beach, South Carolina, USA.

Jarboui, B.; Derbel, H.; Hanafi, S. y Mladenović, N. (2013). Variable Neighborhood Search for Location Routing. Computers \& Operations Research, 40(1), 47-57. https://doi.org/10.1016/j.cor.2012.05.009.

Jemai, J.; Zekri, M. y Mellouli, K. (2012). An NSGA-II Algorithm for the Green Vehicle Routing Problem. En J.-K. HAON y M. MidDENDORF (eds). European conference on evolutionary computation in combinatorial optimization (pp. 37-48). Springer.

Jeon, G.; Leep, H. R. y Shim, J. Y. (2007). A Vehicle Routing Problem Solved by Using a Hybrid Genetic Algorithm. Computers \& Industrial Engineering, 53(4), 680-692. https://doi.org/10.1016/j.cie.2007.06.031.

Jepsen, M.; Spoorendonk, S. y Ropke, S. (2013). A Branch-and-cut Algorithm for the Symmetric Two-echelon Capacitated Vehicle Routing Problem. Transportation Science, 47(1), 23-37. 
Jin, J.; Crainic, T. G. y Løkketangen, A. (2014). A Cooperative Parallel Metaheuristic for the Capacitated Vehicle Routing Problem. Computers \& Operations Research, 44, 33-41. https://doi.org/10.1016/j.cor.2013.10.004.

Kara, I.; Kara, B. Y. y Yetis, M. K. (2007). Energy Minimizing Vehicle Routing Problem. En A. Dress; Y. Xu y B. ZhU (eds). International Conference on Combinatorial Optimization and Applications (pp. 62-71). Springer.

Karakatič, S. y Podgorelec, V. (2015). A Survey of Genetic Algorithms for Solving Multi Depot Vehicle Routing Problem. Applied Soft Computing, 27, 519-532. https://doi.org/10.1016/j.asoc.2014.11.005.

Karaoglan, I. y Altiparmak, F. (2015). A Memetic Algorithm for the Capacitated Location-routing Problem with Mixed Backhauls. Computers \& Operations Research, 55, 200-216. https://doi.org/10.1016/j.cor.2014.06.009.

Karapetyan, D. y Gutin, G. (2011). Lin-Kernighan Heuristic Adaptations for the Generalized Traveling Salesman Problem. European Fournal of Operational Research, 208(3), 221-232. https://doi.org/10.1016/j.ejor.2010.08.011.

KARP, R. (1977). Probabilistic Analysis of Partitioning Algorithms for the Traveling Salesman Problem in the Plane. Mathematics of Operations Research, 2(3), 209-224. https://doi.org/10.1287/moor.2.3.209.

Kent, J. y Mudford, N. (1979). Motor Vehicle Emissions and Fuel Consumption Modelling. Transportation Research Part A: General, 13(6), 395-406. https://doi.org/10.1016/0191-2607(79)90003-7.

Kuincewicz, J. G.; Luss, H. y Pilcher, M. G. (1990). Fleet Size Planning When Outside Carrier Services are Available. Transportation Science, 24(3), 169-182. https://doi.org/10.1287/trsc.24.3.169.

KLose, A. y GorTz, S. (2007). A Branch and Price Algorithm for the Capacitated Facility Location Problem. European Journal of Operational Research, 179, 1109-1125. https://doi.org/10.1016/j.ejor.2005.03.078.

Kouridis, C.; Gkatzoflias, D.; Kioutsioukis, I.; NtZiachristos, L.; Pastorello, C. y Dilara, P. (2010). Uncertainty Estimates and Guidance for Road Transport Emission Calculations. [Reporte técnico EUR 24296 EN]. Publications Office of the European Union.

Kovacs, A. A.; Golden, B. L.; Hartl, R. F. y Parragh, S. N. (2014). Vehicle Routing Problems in Which Consistency Considerations are Important: A Survey. Networks, 64(3), 192-213. https://doi.org/10.1002/net.21565.

Krajewska, M. A. y Kopfer, H. (2009). Transportation Planning in Freight Forwarding Companies: Tabu Search Algorithm for the Integrated Operational Transportation Planning Problem. European fournal of Operational Research, 197(2), 741-751. https://doi.org/10.1016/j.ejor.2008.06.042.

KraticA, J.; Kostić, T.; Tošić, D.; Dugošija, D. y Filipović, V. (2012). A Genetic Algorithm for the Routing and Carrier Selection Problem. Computer Science and Information Systems, 9(1), 49-62. https://doi.org/10.2298/CSIS100425067K.

Kucukoglu, I.; Ene, S.; Aksoy, A. y Ozturk, N. (2013). Green Capacitated Vehicle Routing Problem Fuel Consumption Optimization Model. Computational Engineering Research, 3, 16-23.

Kuo, Y. (2010). Using Simulated Annealing to Minimize Fuel Consumption for the Time Dependent Vehicle Routing Problem. Computers \& Industrial Engineering, 59, 157-165. https://doi.org/10.1016/j.cie.2010.03.012.

Kwon, Y.-J.; CHoI, Y.-J. y LeE, D.-H. (2013). Heterogeneous Fixed Fleet Vehicle Routing Considering Carbon Emission. Transportation Research Part D: Transport and Environment, 23, 81-89. https://doi.org/10.1016/j.trd.2013.04.001.

LaI, C.-M.; Chiu, C.-C.; Liu, W.-C. y Yeh, W.-C. (2019). A Novel Nondominated Sorting Simplified Swarm Optimization for Multi-stage Capacitated Facility Location Problems with Multiple Quantitative and Qualitative Objectives. Applied Soft Computing, 84, 1-17. https://doi.org/10.1016/j.asoc.2019.105684.

Lalla-Ruiz, E.; Expósito-Izquierdo, C.; Taheripour, S. y Voss, S. (2016). An Improved Formulation for the Multidepot Open Vehicle Routing Problem. OR spectrum, 38(1), 175-187. https://doi.org/10.1007/s00291-015-0408-9.

Lam, M.; Mittenthal, J. y Gray, B. (2009). The Impact of Stopping Rules on Hierarchical Capacitated Clustering in Location Routing Problems. Journal of Management Information and Decision Sciences, 12(1/2), 13-28. 
LAporte, G. (1992). The Vehicle Routing Problem: An Overview of Exact and Approximate Algorithms. European journal of operational research, 59(3), 345-358. https://doi.org/10.1016/0377-2217(92)90192-C.

Laporte, G.; Louveaux, F. V. y Van Hamme, L. (2002). An Integer L-shaped Algorithm for the Capacitated Vehicle Routing Problem with Stochastic Demands. Operations Research, 50(3), 415-423. https://doi.org/10.1287/opre.50.3. 415.7751 .

Laporte, G. y Semet, F. (2002). The Vehicle Routing Problem. Society for Industrial and Applied Mathematics.

Lavorato, M.; Franco, J. F.; Rider, M. J. y Romero, R. (2012). Imposing Radiality Constraints in Distribution System Optimization Problems. IEEE Transactions on Power Systems, 27(1), 172-180. https://doi.org/10.1109/TPWRS.2011. 2161349.

Lee, C.-G.; Epelman, M. A.; White III, C. C. y Bozer, Y. A. (2006). A Shortest Path Approach to the Multiple-vehicle Routing Problem with Split Pick-ups. Transportation research part B: Methodological, 40(4), 265-284. https://doi.org/ 10.1016/j.trb.2004.11.004.

Leung, J. y Magnanti, T. (1989). Valid Inequalities and Facets of the Capacitated Plant Location Problem. Mathematical Programming, 44, 271-291. https://doi.org/10.1007/BF01587093.

LI, H.; Yuan, J.; Lv, T. y Chang, X. (2016a). The Two-echelon Time-constrained Vehicle Routing Problem in Linehaul-delivery Systems Considering Carbon Dioxide Emissions. Transportation Research Part D: Transport and Environment, 49, 231-245. https://doi.org/10.1016/j.trd.2016.10.002.

LI, J.; Chu, F.; Prins, C. y Zhu, Z. (2014). Lower and Upper Bounds for a Two Stage Capacitated Facility Location Problem with Handling Costs. European fournal of Operational Research, 236, 857-967. https://doi.org/10.1016/j.ejor. 2013.10.047.

Li, J.; Li, Y. y Pardalos, P. M. (2016b). Multi-depot Vehicle Routing Problem with Time Windows Under Shared Depot Resources. Fournal of Combinatorial Optimization, 31(2), 515-532. https://doi.org/10.1007/s10878-014-9767-4.

Li, J.; Wang, D. y Zhang, J. (2018). Heterogeneous Fixed Fleet Vehicle Routing Problem Based on Fuel and Carbon Emissions. Journal of Cleaner Production, 201, 896-908. https://doi.org/10.1016/j.jclepro.2018.08.075.

Liang, X.; De Almeida Correia, G. H.; An, K. y van Arem, B. (2020). Automated Taxis' Dial-a-ride Problem with Ride-sharing Considering Congestion-based Dynamic Travel Times. Transportation Research Part C: Emerging Technologies, 112, 260-281. https://doi.org/10.1016/j.trc.2020.01.024.

Lin, C.; Choy, K. L.; Ho, G. T.; Chung, S. H. y Lam, H. (2014). Survey of Green Vehicle Routing Problem: Past and Future Trends. Expert systems with applications, 41(4), 1118-1138. https://doi.org/10.1016/j.eswa.2013.07.107.

Lin, C. y Kwok, R. (2006). Multi-objective Metaheuristics for a Location-routing Problem with Multiple Use of Vehicles on Real Data and Simulated Data. European journal of operational research, 175(3), 1833-1849. https: //doi.org/10.1016/j.ejor.2004.10.032.

LiN, S. (1965). Computer Solutions of the Traveling Salesman Problem. Bell System Technical fournal, 44(10), 22452269. https://doi.org/10.1002/j.1538-7305.1965.tb04146.x.

Lin, S. y Kernighan, B. W. (1973). An Effective Heuristic Algorithm for the Traveling-salesman Problem. Operations research, 21(2), 498-516. https://doi.org/10.1287/opre.21.2.498.

Lin, S.-W.; LeE, Z.-J.; YIng, K.-C. y LeE, C.-Y. (2009). Applying Hybrid Meta-heuristics for Capacitated Vehicle Routing Problem. Expert Systems with Applications, 36(2), 1505-1512. https://doi.org/10.1016/j.eswa.2007.11.060.

Liu, R. y Jiang, Z. (2012). The Close-open Mixed Vehicle Routing Problem. European fournal of Operational Research, 220(2), 349-360. https://doi.org/10.1016/j.ejor.2012.01.061 
Liu, R.; Jiang, Z. y Geng, N. (2014). A Hybrid Genetic Algorithm for the Multi-depot Open Vehicle Routing Problem. OR spectrum, 36(2), 401-421. https://doi.org/10.1007/s00291-012-0289-0.

Liu, S. (2013). A Hybrid Population Heuristic for the Heterogeneous Vehicle Routing Problems. Transportation Research Part E: Logistics and Transportation Review, 54, 67-78. https://doi.org/10.1016/j.tre.2013.03.010.

Lopes, R. B.; Barreto, S.; Ferreira, C. y Santos, B. S. (2008). A Decision-support Tool for a Capacitated Locationrouting Problem. Decision Support Systems, 46(1), 366-375. https://doi.org/10.1016/j.dss.2008.07.007.

Lorena, L. A. y Senne, E. L. (2004). A Column Generation Approach to Capacitated P-median Problems. Computers \& Operations Research, 31(6), 863-876. https://doi.org/10.1016/S0305-0548(03)00039-X.

Luo, Z; Liv, Z. y SHI, J. (2017). A Two-echelon Cooperated Routing Problem for a Ground Vehicle and Its Carried Unmanned Aerial Vehicle. Sensors, 17(5). Artículo 1144. https://doi.org/10.3390/s17051144.

Marinakis, Y. (2015). An Improved Particle Swarm Optimization Algorithm for the Capacitated Location Routing Problem and for the Location Routing Problem with Stochastic Demands. Applied Soft Computing, 37, 680-701. https://doi.org/10.1016/j.asoc.2015.09.005.

Marinakis, Y. y MarinaKi, M. (2008a). A Bilevel Genetic Algorithm for a Real Life Location Routing Problem. International Journal of Logistics: Research and Applications, 11(1), 49-65. https://doi.org/10.1080/ 13675560701410144 .

Marinakis, Y. y Marinaki, M. (2008b). A Particle Swarm Optimization Algorithm with Path Relinking for the Location Routing Problem. fournal of Mathematical Modelling and Algorithms, 7(1), 59-78. https://doi.org/10.1007/ s10852-007-9073-6.

Marinakis, Y. y Marinaki, M. (2010). A Hybrid Genetic-Particle Swarm Optimization Algorithm for the Vehicle Routing Problem. Expert Systems with Applications, 37(2), 1446-1455. https://doi.org/10.1016/j.eswa.2009.06.085.

Marler, R. T. y Arora, J. S. (2009). Multi-objective Optimization: Concepts and Methods for Engineering. VDM Publishing.

Matl, P.; Hartu, R. F. y Vidal, T. (2019). Workload Equity in Vehicle Routing: The Impact of Alternative Workload Resources. Computers \& Operations Research, 110, 116-129. https://doi.org/10.1016/j.cor.2019.05.016.

McKinnon, A. C. y Woodburn, A. (1996). Logistical Restructuring and Road Freight Traffic Growth. Transportation, 23(2), 141-161. https://doi.org/10.1007/BF00170033.

MehrJerdi, Y. Z. y Nadizadeh, A. (2013). Using Greedy Clustering Method to Solve Capacitated Location-routing Problem with Fuzzy Demands. European fournal of Operational Research, 229(1), 75-84. https://doi.org/10.1016/j. ejor.2013.02.013.

Miettinen, K. (1999). Nonlinear Multiobjective Optimization. Kluwer Academic Publishers.

Miller, C. E.; Tucker, A. W. y Zemlin, R. A. (1960). Integer Programming Formulation of Traveling Salesman Problems. Journal of the ACM (JACM), 7(4), 326-329. https://doi.org/10.1145/321043.321046.

Mirabi, M.; Ghomi, S. F. y Jolai, F. (2010). Efficient Stochastic Hybrid Heuristics for the Multi-depot Vehicle Routing Problem. Robotics and Computer-Integrated Manufacturing, 26(6), 564-569. https://doi.org/10.1016/j.rcim.2010.06. 023.

Mohamed, I. B.; Klibi, W.; Sadykov, R.; Şen, H. y Vanderbeck, F. (2019). A Benders Decomposition Approach for the Two-Echelon Stochastic Multi-period Capacitated Location-Routing Problem. (Nro. hal-02178459). HAL. https: //hal.archives-ouvertes.fr/hal-02178459.

Montoya-Torres, J. R.; Franco, J. L.; Isaza, S. N.; Jiménez, H. F. y Herazo-Padilla, N. (2015). A Literature Review on the Vehicle Routing Problem with Multiple Depots. Computers \& Industrial Engineering, 79, 115-129. https: //doi.org/10.1016/j.cie.2014.10.029. 
Mufalli, F.; Batta, R. y Nagi, R. (2012). Simultaneous Sensor Selection and Routing of Unmanned Aerial Vehicles for Complex Mission Plans. Computers \& Operations Research, 39(11), 2787-2799. https://doi.org/10.1016/j.cor.2012. 02.010 .

Naddef, D. y Rinaldi, G. (2002). Branch-and-cut Algorithms for the Capacitated Vehicle Routing Problem. En P. Tотн y D. Vigo (eds). The Vehicle Routing Problem (pp. 53-84). Society for Industrial and Applied Mathematics.

Nadizadeh, A. y Kafash, B. (2019). Fuzzy Capacitated Location-routing Problem with Simultaneous Pickup and Delivery Demands. Transportation Letters, 11(1), 1-19. https://doi.org/10.1080/19427867.2016.1270798.

Nagy, G. y Salhi, S. (2005). Heuristic Algorithms for Single and Multiple Depot Vehicle Routing Problems with Pickups and Deliveries. European journal of operational research, 162(1), 126-141. https://doi.org/10.1016/j.ejor.2002. 11.003 .

NAuss, R. (1978). An Improved Algorithm for the Capacitated Facility Location Problem. The fournal of the Operational Research Society, 29(12), 1195-1201. https://doi.org/10.1057/jors.1978.263.

NAzIF, H. y LeE, L. S. (2012). Optimised Crossover Genetic Algorithm for Capacitated Vehicle Routing Problem. Applied Mathematical Modelling, 36(5), 2110-2117. https://doi.org/10.1016/j.apm.2011.08.010.

Norback, J. P. y Love, R. F. (1977). Geometric Approaches to Solving the Traveling Salesman Problem. Management Science, 23(11), 1208-1223. https://www.jstor.org/stable/2630660.

Nuortio, T.; Кутојокi, J.; Niska, H. y Braysy, O. (2006). Improved Route Planning and Scheduling of Waste Collection and Transport. Expert Systems with Applications, 30, 223-232. http://dx.doi.org/10.1016/j.eswa.2005.07. 009 .

Ocampo, E. M. T.; Castaño, A. H. D. y Zuluaga, A. H. E. (2016). Desempeño de las técnicas de agrupamiento para resolver el problema de ruteo con múltiples depósitos. TecnoLógicas, 19(36), 49-62. https://doi.org/10.22430/ 22565337.593.

Ocampo, E. M. T.; Guimarãesb, F. G. y Rendónc, R. A. G. (2017). Introducing Radiality Constraints in Capacitated Location-routing Problems. International fournal of Industrial Engineering Computations, 8(4), 441-452. https://doi. org/10.5267/j.jijiec.2017.3.004.

OEE (2015). Fuel Consumption Guide. [Reporte técnico 2015]. Natural Resources Canada.

Orlis, C.; Laganá, D.; Dullaert, W. y Vigo, D. (2020). Distribution with Quality of Service Considerations: The Capacitated Routing Problem with Profits and Service Level Requirements. Omega, 93, 1-18. https://doi.org/10. 1016/j.omega.2019.02.003.

Osaba, E.; Carballedo, R.; Diaz, F. y Perallos, A. (2013). Discussion Related to "Wang, C.-H., \& Lu, J.Z.(2009). A Hybrid Genetic Algorithm That Optimizes Capacitated Vehicle Routing Problem. Expert Systems with Applications, 36 (2), 2921-2936”. Expert Systems with Applications, 40(14), 5425-5426. https://doi.org/10.1016/j.eswa. 2013.04.008.

Osman, I. (1991). Metastrategy Simulated Annealing and Tabu Search Algorithms Forcombinatorial Optimization Problems. [Tesis Doctoral, The Management School, Imperial College]. Repositorio Imperial College London. https:/spiral.imperial.ac.uk/bitstream/10044/1/7596/1/Ibrahim_Hassan_Osman-1991-PhD-Thesis.pdf.

Ospina-Toro, D.; Toro-Ocampo, E. M. y Gallego-Rendón, R. A. (2017). Metodología para crear rutas alimentadoras en sistemas de transporte masivo. Revista Facultad de Ingeniería, 26(45), 9-21.

Ozgen, D. y Gulsun, B. (2014). Combining Possibilistic Linear Programming and Fuzzy Ahp for Solving the Multiobjective Capacitated Multi-facility Location Problem. Information Sciences, 268, 185-201. https://doi.org/10.1016/j. ins.2014.01.024. 
Özyurt, Z. y AKsEn, D. (2007). Solving the Multi-depot Location-routing Problem with Lagrangian Relaxation. En E. K. BAKer; A. Joseph; A. Mehrotra y M. A. Trick (eds). Extending the horizons: Advances in computing, optimization, and decision technologies (pp. 125-144). Springer.

Pan, S.; Ballot, E. y Fontane, F. (2013). The Reduction of Greenhouse Gas Emissions From Freight Transport by Pooling Supply Chains. International journal of production economics, 143(1), 86-94. https://doi.org/10.1016/j.ijpe. 2010.10.023

Pekel, E. y Kara, S. S. (2019). Solving Fuzzy Capacitated Location Routing Problem Using Hybrid Variable Neighborhood Search and Evolutionary Local Search. Applied Soft Computing, 83. Artículo 105665. https://doi. org/10.1016/j.asoc.2019.105665.

Peng, Z.; Manier, H. y Manier, M.-A. (2017). Particle Swarm Optimization for Capacitated Location-routing Problem. IFAC-PapersOnLine, 50(1), 14668-14673. https://doi.org/10.1016/j.ifacol.2017.08.2495.

Penna, P. H. V.; Subramanian, A. y Ochi, L. S. (2013). An Iterated Local Search Heuristic for the Heterogeneous Fleet Vehicle Routing Problem. Fournal of Heuristics, 19(2), 201-232. https://doi.org/10.1007/s10732-011-9186-y.

Perboli, G.; Rosano, M.; Saint-Guillain, M. y Rizzo, P. (2018a). Simulation-Optimisation Framework for City Logistics: An Application on Multimodal Last-mile Delivery. IET Intelligent Transport Systems, 12(4), 262-269. https://doi.org/10.1049/iet-its.2017.0357.

Perboli, G.; Tadei, R. y Fadda, E. (2018b). New Valid Inequalities for the Two-echelon Capacitated Vehicle Routing Problem. Electronic Notes in Discrete Mathematics, 64, 75-84. https://doi.org/10.1016/j.endm.2018.01.009.

Perboli, G.; Tadei, R. y Vigo, D. (2011). The Two-echelon Capacitated Vehicle Routing Problem: Models and Mathbased Heuristics. Transportation Science, 45(3), 364-380. https://doi.org/10.1287/trsc.1110.0368.

Pereira, F. B.; Tavares, J.; Machado, P. y Costa, E. (2002). GVR: a New Genetic Representation for the Vehicle Routing Problem. En M. O’Neill; R. F. E. Sutcliffe; C. Ryan; M. Eaton y N. J. L. Griffith (eds). Irish Conference on Artificial Intelligence and Cognitive Science (pp. 95-102). Springer.

PerL, J. y Daskin, M. S. (1984). A Unified Warehouse Location-routing Methodology. Journal of Business Logistics, 5(1), 92-111.

Pirkul, H. (1987). Efficient Algorithms for the Capacitated Concentrator Location Problem. Computers \& Operations Research, 14, 197-208. https://doi.org/10.1016/0305-0548(87)90022-0.

Pirkwieser, S. y Raidl, G. R. (2010). Variable Neighborhood Search Coupled with ILP-based Very Large Neighborhood Searches for the (periodic) Location-routing Problem. En M. J. BLESA; C. BLum; G. RaIdL; A. Roli y M. SAMPELS (eds). International Workshop on Hybrid Metaheuristics (pp. 174-189). Springer.

Ponboon, S.; Qureshi, A. G. y TAniguchi, E. (2016). Branch-and-price Algorithm for the Location-routing Problem with Time Windows. Transportation Research Part E: Logistics and Transportation Review, 86, 1-19. https://doi.org/ 10.1016/j.tre.2015.12.003.

Pooley, J. (1994). Integrated Production and Distribution Facility Planning at Ault Foods. Interfaces, 24(4), 113-121. https://doi.org/10.1287/inte.24.4.113.

Potvin, J. y Rousseau, M. (1990). A Parallel Route Building Vehicle Routing and Scheduling Problem with Time Windows. European fournal Operation Research, 66(3), 331-340. https://doi.org/10.1016/0377-2217(93)90221-8.

Potvin, J.-Y. y Naud, M.-A. (2011). Tabu Search with Ejection Chains for the Vehicle Routing Problem with Private Fleet and Common Carrier. Journal of the operational research society, 62(2), 326-336. https://doi.org/10.1057/jors. 2010.102 . 
Pourreza, P.; Tavakkoli-Moghaddam, R.; Aghamohamadi, S.; Bozorgi-Amiri, A. y Rahimi, Y. (2017). A Capacitated Location-routing Problem with Customer Satisfaction Under Facility Disruption. En: 2017 IEEE International Conference on Industrial Engineering and Engineering Management (IEEM) (pp. 979-983). IEEE. https: //doi.org/10.1109/IEEM.2017.8290038.

Pradenas, L.; Oportus, B. y Parada, V. (2013). Mitigation of Greenhouse Gas Emissions in Vehicle Routing Problems with Backhauling. Expert Systems with Applications, 40(8), 2985-2991. https://doi.org/10.1016/j.eswa.2012.12.014.

Prins, C. (2004). A Simple and Effective Evolutionary Algorithm for the Vehicle Routing Problem. Computers \& operations research, 31(12), 1985-2002. https://doi.org/10.1016/S0305-0548(03)00158-8.

Prins, C.; Prodhon, C. y Calvo, R. W. (2006). Solving the Capacitated Location-routing Problem by a GRASP Complemented by a Learning Process and a Path Relinking. 4OR, 4(3), 221-238. https://doi.org/10.1287/trsc.1060. 0187.

Prins, C.; Prodhon, C.; Ruiz, A.; Soriano, P. y Wolfler Calvo, R. (2007). Solving the Capacitated Location-routing Problem by a Cooperative Lagrangean Relaxation-granular Tabu Search Heuristic. Transportation Science, 41(4), 470-483. https://doi.org/10.1287/trsc.1060.0187.

Prodhon, C. (2011). A Hybrid Evolutionary Algorithm for the Periodic Location-routing Problem. European fournal of Operational Research, 210(2), 204-212. https://doi.org/10.1016/j.ejor.2010.09.021.

Prodhon, C. y Prins, C. (2008). A Memetic Algorithm with Population Management (MA| PM) for the Periodic Location-routing Problem. En M. J. Blesa; C. Blum; C. Cotta; A. J. Fernández; J. E. Gallardo; A. Roli y M. SAMPELS (eds). International Workshop on Hybrid Metaheuristics (pp. 43-57). Springer.

Prodhon, C. y Prins, C. (2014). A Survey of Recent Research on Location-routing Problems. European fournal of Operational Research, 238(1), 1-17. https://doi.org/10.1016/j.ejor.2014.01.005.

QIan, J. y Eglese, R. (2016). Fuel Emissions Optimization in Vehicle Routing Problems with Time-varying Speeds. European Fournal of Operational Research, 248(3), 840-848. https://doi.org/10.1016/j.ejor.2015.09.009.

Quintero-Araujo, C. L.; Caballero-Villalobos, J. P.; Juan, A. A. y Montoya-Torres, J. R. (2017). A Biasedrandomized Metaheuristic for the Capacitated Location Routing Problem. International Transactions in Operational Research, 24(5), 1079-1098.

Quintero-Araujo, C. L.; Guimarans, D. y Juan, A. A. (2019). A Simheuristic Algorithm for the Capacitated Location Routing Problem with Stochastic Demands. Fournal of Simulation, O(0), 1-18. https://doi.org/10.1080/17477778.2019. 1680262 .

Rahmani, A. y MirHassani, M. (2014). A Hybrid Firefly-Genetic Algorithm for the Capacitated Facility Location Problem. Expert Systems with Applications, 283, 70-78. https://doi.org/10.1016/j.ins.2014.06.002.

Rahmani, Y.; Ramdane Cherif-Khettaf, W. y Oulamara, A. (2016). The Two-echelon Multi-products Locationrouting Problem with Pickup and Delivery: Formulation and Heuristic Approaches. International fournal of Production Research, 54(4), 999-1019. https://doi.org/10.1080/00207543.2015.1040127.

Ramos, T. R. P.; Gomes, M. I. y PóvoA, A. P. B. (2020). Multi-depot Vehicle Routing Problem: a Comparative Study of Alternative Formulations. International fournal of Logistics Research and Applications, 23(2), 103-120. https://doi. org/10.1080/13675567.2019.1630374.

Reisman, A. (1992). Management Science Knowledge: Its Creation, Generalization, and Consolidation. Quorum Books.

Rolland, E.; Schilling, D. y Current, J. (1996). An Efficient Tabu Search Procedure for the Pmedian Problem. European fournal of Operational Research, 96, 329-342. 
Romilly, P. (1999). Substitution of Bus for Car Travel in Urban Britain: An Economic Evaluation of Bus and Car Exhaust Emission and Other Costs. Transportation Research Part D: Transport and Environment, 4(2), 109-125. https: //doi.org/10.1016/S1361-9209(98)00028-5.

Ryan, D. y Foster, E. (1981). An Integer Programming Approach to Scheduling. En A. Wren (ed). Computer Scheduling of Public Transport (pp. 269-280). North-Holland Publishing Company.

SALHI, S. y SARI, M. (1997). A Multi-level Composite Heuristic for the Multi-depot Vehicle Fleet Mix Problem. European Journal of Operational Research, 103(1), 95-112. https://doi.org/10.1016/S0377-2217(96)00253-6.

Samuel, R. (1983). Routing and Scheduling of Vehicles and Crews: the State of the Art. Computers \& Operations Research, 10(2), 63-211. https://doi.org/10.1016/0305-0548(83)90030-8.

Sawadsitang, S.; Jiang, S.; Niyato, D. y Wang, P. (2017a). Optimal Stochastic Package Delivery Planning with Deadline: A Cardinality Minimization in Routing. En: 2017 IEEE 86th Vehicular Technology Conference (VTC-Fall) (pp. 1-7). IEEE. https://doi.org/10.1109/VTCSpring.2017.8108576.

SAWAdsitang, S.; KaEwPuAng, R.; JiAng, S.; NiYato, D. y Wang, P. (2017b). Optimal Stochastic Delivery Planning in Full-truckload and Less-than-truckload Delivery. En: 2017 IEEE 85th Vehicular Technology Conference (VTC Spring) (pp. 1-5). IEEE.

Schingh, M.; Brunet, É. y Gosselin, P. (2000). Canadian New Light Duty Vehicles: Trends in Fuel Consumption and Characteristics (1988-1998). Transportation Energy Use Division, Office of Energy Efficiency, Natural Resources Canada.

Schittekat, P. y Sörensen, K. (2009). OR Practice-Supporting 3PL Decisions in the Automotive Industry by Generating Diverse Solutions to a Large-scale Location-routing Problem. Operations research, 57(5), 1058-1067. https://doi.org/10.1287/opre.1080.0633.

Schotт, J. R. (1995). Fault Tolerant Design Using Single and Multicriteria Genetic Algorithm Optimization. [Tesis de maestría, Air Force Inst of Tech Wright-Patterson AFB OH]. Repositorio United State Air Force Academy. https: //dspace.mit.edu/bitstream/handle/1721.1/11582/33945300-MIT.pdf?sequence=2.

Scora, G. y Barth, M.). Comprehensive Modal Emissions Model (cmem), version 3.01. [User guide]. Centre for Environmental Research and Technology. University of California, Riverside.

Secomandi, N. y Margot, F. (2009). Reoptimization Approaches for the Vehicle-routing Problem with Stochastic Demands. Operations research, 57(1), 214-230. https://doi.org/10.1287/opre.1080.0520.

SendeCO2 (2014). Unit Allowances (EUA) and Carbon Credits (CER'S). SendeCO2.

Seyyedhasani, H. y Dvorak, J. S. (2017). Using the Vehicle Routing Problem to Reduce Field Completion Times with Multiple Machines. Computers and Electronics in Agriculture, 134, 142-150. https://doi.org/10.1016/j.compag.2016. 11.010 .

Shetty, B. (1990). Approximate Solutions to Large Scale Capacitated Facility Location Problems. Applied Mathematics and Computation, 39, 159-175. https://doi.org/10.1016/0096-3003(90)90029-3.

Smilowitz, K.; NowaK, M. y Jiang, T. (2013). Workforce Management in Periodic Delivery Operations. Transportation Science, 47(2), 214-230. https://doi.org/10.1287/trsc.1120.0407.

Soysal, M.; Bloemhof-Ruwaard, J. M. y Bektaş, T. (2015). The Time-dependent Two-echelon Capacitated Vehicle Routing Problem with Environmental Considerations. International fournal of Production Economics, 164, 366-378. https://doi.org/10.1016/j.ijpe.2014.11.016.

Stenger, A.; Schneider, M. y Enz, S. (2011). A Hybrid Grasp-Vns Algorithm With Efective Depot Reduction Mechanism For The Capacitated Location Routing Problem. Reporte técnico. Goethe University Frankfurt. 
Stenger, A.; Vigo, D.; EnZ, S. y Schwind, M. (2013). An Adaptative Variable Neighboorhod Search Algorithm for a Vehicle Routing Problem Arising in Small Package Shipping. Transportation Science, 47(1), 64-80.

Subramanian, A. (2012). Heuristic, Exact and Hybrid Approaches for Vehicle Routing Problems. [Tesis Doctoral, Universidade Federal Fluminense, Niteroi, Sao Pablo].

Sungur, I.; Ren, Y.; OrdóñEZ, F.; Dessouky, M. y Zhong, H. (2010). A Model and Algorithm for the Courier Delivery Problem with Uncertainty. Transportation science, 44(2), 193-205. https://doi.org/10.1287/trsc.1090.0303.

Surekha, P. y Sumathi, S. (2011). Solution To Multi-Depot Vehicle Routing Problem Using Genetic Algorithms. World Applied Programming, 1(3), 118-131. http://waprogramming.com/papers/50ae4ba59c2fa7.48950811.pdf.

Suzuki, Y. (2011). A New Truck-routing Approach for Reducing Fuel Consumption and Pollutants Emission. Transportation Research Part D: Transport and Enviromental, 16(1), 73-77. https://doi.org/10.1016/j.trd.2010.08.003.

Taillard, É. (1993). Parallel Iterative Search Methods for Vehicle Routing Problems. Networks, 23(8), 661-673. https: //doi.org/10.1002/net.3230230804.

Taillard, É. D.; Gambardella, L. M.; Gendreau, M. y Potvin, J.-Y. (2001). Adaptive Memory Programming: A Unified View of Metaheuristics. European fournal of Operational Research, 135(1), 1-16. https://doi.org/10.1016/ S0377-2217(00)00268-X.

Tarantilis, C. D.; Ioannou, G.; Kiranoudis, C. T. y Prastacos, G. P. (2005). Solving the Open Vehicle Routeing Problem Via a Single Parameter Metaheuristic Algorithm. Fournal of the Operational Research Society, 56(5), 588596. https://doi.org/10.1057/palgrave.jors.2601848.

Tarantilis, C. D. y Kiranoudis, C. T. (2001). Using the Vehicle Routing Problem for the Transportation of Hazardous Materials. Operational Research, 1. Artículo nro. 67. https://doi.org/10.1007/BF02936400.

Taslimi, M.; Batta, R. y Kwon, C. (2020). Medical Waste Collection Considering Transportation and Storage Risk. Computers \& Operations Research, 120, 1-17. https://doi.org/10.1016/j.cor.2020.104966.

Tavakkoli-Moghaddam, R.; Makui, A. y Mazloomi, Z. (2010). A New Integrated Mathematical Model for a Biobjective Multi-depot Location-routing Problem Solved by a Multi-objective Scatter Search Algorithm. Fournal of Manufacturing Systems, 29(2-3), 111-119. https://doi.org/10.1016/j.jmsy.2010.11.005.

Thompson, P. y Psaraftis, H. (1993). Cyclic Transfer Algorithms for Multivehicle Routing and Scheduling Problems. Operations Research, 41, 935-946. https://doi.org/10.1287/opre.41.5.935.

Tian, Y.; Cheng, R.; Zhang, X. y Jin, Y. (2017). PLATEMO: A MATLAB Platform for Evolutionary Multi-objective Optimization. IEEE Computational Intelligence Magazine, 12(4), 73-87. https://doi.org/10.1109/MCI.2017.2742868.

Tillman, F. A. (1969). The Multiple Terminal Delivery Problem with Probabilistic Demands. Transportation Science, 3(3), 192-204. https://doi.org/10.1287/trsc.3.3.192.

Tillman, F. A. y Cain, T. M. (1972). An Upperbound Algorithm for the Single and Multiple Terminal Delivery Problem. Management Science, 18(11), 664-682. https://doi.org/10.1287/mnsc.18.11.664.

Toro, E.; Franco, J.; Echeverri, M.; Guimarães, F. y Rendón, R. (2017a). Green Open Location-routing Problem Considering Economic and Environmental Costs. International fournal of Industrial Engineering Computations, 8(2), 203-216. https://doi.org/10.5267/j.jijiec.2016.10.001.

Toro, E. M.; Franco, J. F.; Echeverri, M. G. y Guimarães, F. G. (2017b). A Multi-objective Model for the Green Capacitated Location-routing Problem Considering Environmental Impact. Computers \& Industrial Engineering, 110, 114-125. https://doi.org/10.1016/j.cie.2017.05.013.

Toro O, E. M.; Escobar Z, A. H. y Granada E, M. (2016). Literature Review on the Vehicle Routing Problem in the Green Transportation Context. Luna Azul, (42), 362-387. https://doi.org/10.17151/luaz.2016.42.21. 
Toro-Ocampo, E. M.; Franco-Baquero, J. F. y Gallego-Rendón, R. A. (2016). Modelo matemático para resolver el problema de localización y ruteo con restricciones de capacidad considerando flota propia y subcontratada. Ingeniería, Investigación y Tecnología, 17(3), 357-369. https://doi.org/10.1016/j.riit.2016.07.006.

Toth, P. y Vigo, D. (2002). An Overview of Vehicle Routing Problems. En P. Tотн y D. Vigo (eds). The vehicle routing problem (pp. 1-26). SIAM.

Tотн, P. y Vigo, D. (2003). The Granular Tabu Search and Its Application to the Vehicle-routing Problem. Informs Journal on computing, 15(4), 333-346.

Trigeorgis, L. y Mason, S. (1987). Valuing Managerial Operating Flexibility. Midland Corporate finance fournal, 5(1), 14-21.

Tuzun, D. y Burke, L. (1999). A Two-phase Tabu Search Approach to the Location Routing Problem. European fournal of Operational Research, 116(1), 87-99. http://dx.doi.org/10.1016/S0377-2217(98)00107-6.

Ubeda, S.; Arcelus, F. J. y Faulin, J. (2011). Green Logistics at Eroski: A Case Study. International fournal of Production Economics, 131(1), 44-51. https://doi.org/10.1016/j.ijpe.2010.04.041.

Uchoa, E.; Pecin, D.; Pessoa, A.; Poggi, M.; Vidal, T. y Subramanian, A. (2017). New Benchmark Instances for the Capacitated Vehicle Routing Problem. European fournal of Operational Research, 257(3), 845-858. https://doi.org/ 10.1016/j.ejor.2016.08.012.

Ulukan, Z. y Demircioglu, E. (2015). A Survey of Discrete Facility Location Problems. International fournal of Social Behavioral, Educational, Economic, Business and Industrial Engineering, 9(7), 2487-2492.

UMITRI (2014, 6 de octubre). Large Drop in Fuel Economy in September. Michigan News University of Michigan. http://www.umtri.umich.edu/what-were-doing/news/large-drop-fuel-economy-september.

Van Hentenryck, P.; Bent, R. y Coffrin, C. (2010). Strategic Planning for Disaster Recovery with Stochastic Last Mile Distribution. En A. Lodi; M. MiLano y P. Toth (eds). International conference on integration of artificial intelligence (AI) and operations research (OR) techniques in constraint programming (pp. 318-333). Springer.

Van Hentenryck, P.; Coffrin, C. y Bent, R. (2011, agosto). Vehicle Routing for the Last Mile of Power System Restoration. En: Proceedings of the 17th Power Systems Computation Conference (PSCC'11) (pp. 1-8). Curran Associates, Inc.

Van Roy, T. (1986). A Cross Decomposition Algorithm for Capacitated Facility Location. European fournal of Operational Research, 34, 145-163. https://doi.org/10.1287/opre.34.1.145.

Vansteenwegen, P.; Souffriau, W.; Berghe, G. V. y Van Oudheusden, D. (2009). Metaheuristics for Tourist Trip Planning. En K. Sörensen; M. Sevaux; W. Habenicht y M. Geiger (eds). Metaheuristics in the service industry (pp. 15-31). Springer.

Vidal, T.; Crainic, T.; Gendreau, M.; Lahrichi, N. y ReI, W. (2012). A Hybrid Genetic Algorithm for Multidepot and Periodic Vehicle Routing Problems. Operations Research, 60(3), 611-624. dx.doi.org/10.1287/opre.1120.1048.

VidAL, T.; LAPORTe, G. y MATL, P. (2020). A Concise Guide to Existing and Emerging Vehicle Routing Problem Variants. European fournal of Operational Research, 286(2), 401-416. https://doi.org/10.1016/j.ejor.2019.10.010.

Wang, C.-H. y Lu, J.-Z. (2009). A Hybrid Genetic Algorithm That Optimizes Capacitated Vehicle Routing Problems. Expert Systems with Applications, 36(2), 2921-2936. https://doi.org/10.1016/j.eswa.2008.01.072.

Wang, K.; Lan, S. y Zhao, Y. (2017). A Genetic-algorithm-based Approach to the Two-echelon Capacitated Vehicle Routing Problem with Stochastic Demands in Logistics Service. Journal of the Operational Research Society, 68(11), 1409-1421. https://doi.org/10.1057/s41274-016-0170-7. 
Wang, X.; Sun, X. y Fang, Y. (2005, 12 de octubre). A Two-Phase Hybrid Heuristic Search Approach To The LocationRouting Problem. En: IEEE International Conference On Systems, Man And Cybernetics (pp. 3338-3343). IEEE. https: //doi.org/10.1109/ICSMC.2005.1571661.

Wang, X.; Zhan, L.; Ruan, J. y Zhang, J. (2014). How to Choose "last Mile" Delivery Modes for E-fulfillment. Mathematical Problems in Engineering, 2014. Artículo 417129. https://doi.org/10.1155/2014/417129.

Wang, Y.; Zhang, S.; Assogba, K.; FAn, J.; Xu, M. y Wang, Y. (2018). Economic and Environmental Evaluations in the Two-echelon Collaborative Multiple Centers Vehicle Routing Optimization. Fournal of Cleaner Production, 197, 443-461. https://doi.org/10.1016/j.jclepro.2018.06.208.

While, L.; Hingston, P.; Barone, L. y Huband, S. (2006). A Faster Algorithm for Calculating Hypervolume. IEEE transactions on evolutionary computation, 10(1), 29-38. https://doi.org/10.1109/TEVC.2005.851275.

Wren, A. y Holliday, A. (1972). Computer Scheduling of Vehicles Form One or More Depots to a Number of Delivery Points. Operational Research Quarterly, 13, 333-344. http://www.jstor.org/stable/3007888.

Wu, J. y Azarm, S. (2001). Metrics for Quality Assessment of a Multiobjective Design Optimization Solution Set. Journal of Mechanical Design, 123(1), 18-25. https://doi.org/10.1115/1.1329875.

Wu, T.-H.; Low, C. y BAI, J.-W. (2002). Heuristic Solutions to Multi-depot Location-routing Problems. Computers \& Operations Research, 29(10), 1393-1415. https://doi.org/10.1016/S0305-0548(01)00038-7.

Xiao, Y.; Zhao, Q.; Kaku, I. y Xu, Y. (2012). Development Of A Fuel Consumption Optimization Model For The Capacitated Vehicle Routing Problem. Computers and Operations research, 39(7), 1419-1431. https://doi.org/10.1016/ j.cor.2011.08.013

YAKICI, E. (2016). Solving Location and Routing Problem for UAVs. Computers \& Industrial Engineering, 102, $294-301$.

YAnG, J. y Sun, H. (2015). Battery Swap Station Location-routing Problem with Capacitated Electric Vehicles. Computers \& Operations Research, 55, 217-232. https://doi.org/10.1016/j.cor.2014.07.003.

Yao, B.; Hu, P.; Zhang, M. y Tian, X. (2014). Improved Ant Colony Optimization for Seafood Product Delivery Routing Problem. PROMET-Traffic\&Transportation, 26, 1-10. https://doi.org/10.7307/ptt.v26i1.1478.

YU, B.; YAnG, Z. y YAO, B. (2009). An Improved Ant Colony Optimization for Vehicle Routing Problem. European Journal of Operational Research, 196, 171-176. https://doi.org/10.1016/j.ejor.2008.02.028.

Yu, V. y Lin, S. (2015). A Simulated Annealing Heuristic for the Open Location Routing Problem. Computers and Operations Research, 62, 184-196. https://doi.org/10.1016/j.cor.2014.10.009.

Yu, V.; Lin, S.; Lee, W. y Ting, C. (2010). A Simulated Annealing Heuristic For The Capacitated Location Routing Problem. Computers \& Industrial Engineering, 58, 288-299. https://doi.org/10.1016/j.cor.2014.10.009.

Yuan, W.; Wang, J.; LI, J.; YAn, B. y Wu, J. (2017). Two-Stage Heuristic Algorithm for a New Model of Hazardous Material Multi-depot Vehicle Routing Problem. En: UK Workshop on Computational Intelligence (pp. 362-366). Springer.

Zachariadis, E. y Kiranoudis, C. (2012). An Effective Local Search Approach for the Vehicle Routing Problem with Backhauls. Expert Systems with Applications, 39(3), 3174-3184. https://doi.org/10.1016/j.eswa.2011.09.004

Zhang, Y.; BALDAcci, R.; Sim, M. y TANG, J. (2019). Routing Optimization with Time Windows Under Uncertainty. Mathematical Programming, 175(1-2), 263-305. https://doi.org/10.1007/s10107-018-1243-y.

Zhao, Q.; WANG, W. y De SouzA, R. (2018). A Heterogeneous Fleet Two-echelon Capacitated Location-routing Model for Joint Delivery Arising in City Logistics. International fournal of Production Research, 56(15), 5062-5080. https: //doi.org/10.1080/00207543.2017.1401235. 
Eliana Mirledy Toro Ocampo, John Fredy Castañeda Londoño, Ramón Alfonso Gallego Rendón

Zhou, L.; Baldacci, R.; Vigo, D. y Wang, X. (2018). A Multi-depot Two-echelon Vehicle Routing Problem with Delivery Options Arising in the Last Mile Distribution. European fournal of Operational Research, 265(2), 765-778. https://doi.org/10.1016/j.ejor.2017.08.011.

Zhuge, L.; Tong, L. C.; Wu, H.; Chen, Y. y Zhou, X. (2019). Finding Robust and Consistent Space-time Delivery Paths for Multi-day Vehicle Routing Problem. En: 2019 IEEE Intelligent Transportation Systems Conference (ITSC) (pp. 1355-1360). IEEE. https://doi.org/10.1109/ITSC.2019.8916849. 
Este libro presenta un modelo matemático flexible y una metodología metaheurística que puede ser adaptada a diferentes variantes del problema de enrutamiento. Este se basa en el problema clásico del agente viajero, travel salesman problem (TSP), donde se necesita definir una ruta que visite todos los nodos una única vez. En algunas aplicaciones no se permite que la longitud de ruta exceda un límite de tiempo 0 de distancia, lo que hace necesaria la confección de varias trayectorias 0 ciclos para visitar todo el conjunto de clientes, esto configura el problema de múltiples agentes viajeros, multitravel salesman problem (M-TSP). En ambos problemas, el objetivo es visitar todos los clientes, minimizando la sumatoria de los arcos activos.

Basado en el M-TSP, se define el problema de enrutamiento capacitado Capacitated Vehicle Routing Problem (CVRP), donde uno de los nodos actúa como depósito de mercancía con capacidad ilimitada desde el cual se atienden los clientes dispersos geográficamente, de ellos se conoce la localización y una cantidad de mercancía demandada. Para atenderlos; el recurso disponible es una flota de vehículos con capacidad homogénea. En el evento que se requiera ser más eficiente en las entregas a los clientes finales minimizando, por ejemplo, los tiempos de entrega; se puede tomar la decisión de ubicar otros depósitos o centros de distribución. En esta situación se configura el problema de enrutamiento con múltiples depósitos Multi-Depot Vehicle Routing Problem (MDVRP), en esta variante la localización de los depósitos se ha definido previamente y no es una decisión por tomar. Por el contrario, cuando se debe definir la ubicación de los depósitos y la capacidad de estos; aparece el problema de localización y ruteo capacitado Capacitated Location Routing Problem (CLRP). El modelo matemático y la metodología metaheurística que se discute en este libro pueden ser adaptadas, activando y desactivando variables y restricciones para cada variante del VRP, y es eficiente para encontrar solución a una amplia variedad de problemas de enrutamiento: Problemas de enrutamiento clásicos, Problemas abiertos de enrutamiento sin retorno al depósito, Problemas de enrutamiento considerando flota propia y subcontratada y Problemas de enrutamiento considerando efectos ambientales). 